\title{
GUIDE TO RANGE PLANT COMMUNITY TYPES AND CARRYING CAPACITY FOR THE DRY AND CENTRAL MIXEDWOOD SUBREGIONS IN ALBERTA
}

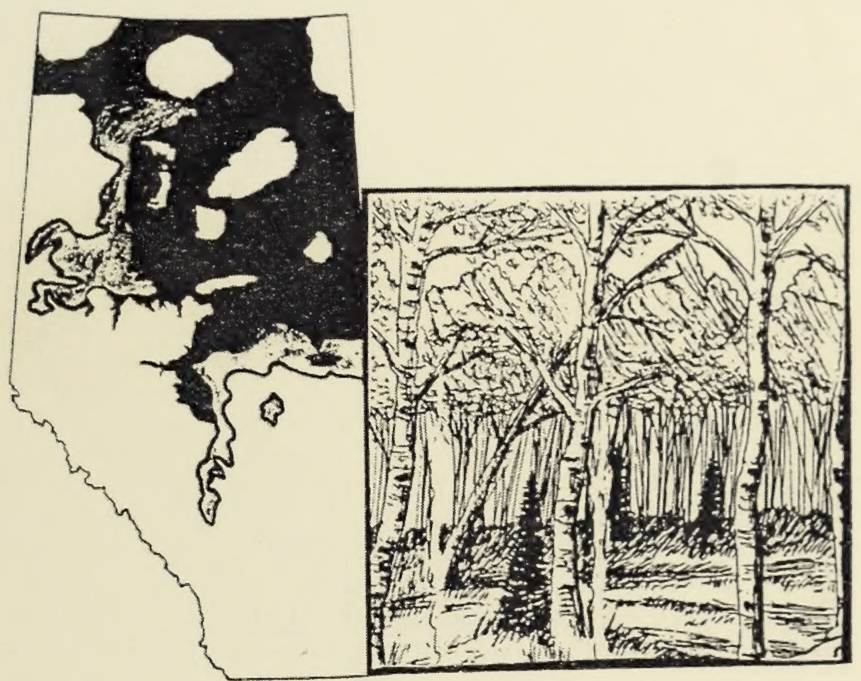

Alborta

SUSTAINABLE RESOURCE DEVELOPMENT

Public Lands $\&$ Forests 
Digitized by the Internet Archive in 2016

https://archive.org/details/guidetorangeplan00will_5 


\section{GUIDE TO RANGE PLANT COMMUNITY TYPES AND CARRYING CAPACITY FOR THE DRY AND CENTRAL MIXEDWOOD SUBREGIONS IN ALBERTA}

\section{Fourth approximation}

(Please note this edition is a revision of the $3^{\text {rd }}$ approximation of the Range Plant Community Types and Carrying Capacity for the Dry and Central Mixedwood Pub. No. T/526)

Prepared by

Michael G. Willoughby, Colin Stone, Carcey Hincz, Darlene Moisey, Gerry Ehlert and

Donna Lawrence 


\section{FORWARD}

In January, 1999 the Rangeland Health Assessment Project was initiated. Its purpose was to coordinate the development of rangeland health assessment methods and ecological site descriptions for both forested and grassland dominated rangelands in the province and transfer the new technology (awareness, information and tools) to livestock producers, staff and other stakeholders. This document "Range plant communities and carrying capacity for the Dry and Central Mixedwood subregions of Alberta, Fourth Approximation" is an effort to organize existing range plant community information for the Boreal Mixedwood subregions into an ecological framework, with the ultimate goal of developing ecological site descriptions as outlined in the Alberta Rangeland Health Task Group, Terms of Reference (1999). This guide encompasses the work of Karen Sundquist (who worked on previous approximations) and Dave Downing who developed the classification for the deciduous communities in the Eastern ecodistricts of the Dry Mixedwood (Downing and Karpuk 1992) and developed a forage gap analysis for the Mixedwood subregions (Downing 2000). It also tries to incorporate the work done by Beckingham and Archibald (1996) on the forested ecosites of the Boreal Mixedwood and work done by Thompson and Hansen (2004) on the lotic and lentic communities of the Mixedwood subregions. As we collect new research information, the fourth approximation will evolve into a range ecological site field guide. One major outcome of the project will be to produce ecological base information which will be used to develop management tools for northern livestock producers, resource managers and other stakeholders of Alberta's Boreal forest. This new knowledge will aide in the sustainable grazing of forested plant communities, and maintain the good health and proper functioning of these ecosystems. 
Pub. no.: T/058

ISBN:0-7785-3555-X

For copies of this report contact:

Michael Willoughby

Public Lands and Forests Division(PLFD)

9920108 st, 9th Floor

Edmonton, Alta.

T5K2M4

(403) 422-4598

E-mail: mike.willoughby@gov.ab.ca

Darlene Moisey

PLFD

St. Paul, Alta.

(780) 645-6308

Darlene.Moisey@.gov.ab.ca

Donna Lawrence

PLFD

Barrhead, Alta.

(780) 374-8231

donna.lawrence@.gov.ab.ca

Colin Stone

PLFD

Peace River, Alta.

(780) 624-6116

colin.stone@.gov.ab.ca
Carcey Hincz

PLFD

Grande Prairie

(780)538-8026

Carcey.Hincz@gov.ab.ca 


\section{Table of contents}

Introduction

Climate of Dry and Central Mixedwood subregions 1

Approach and Methods $\quad 3$

Range Management Concepts 3

Ecologically sustainable stocking rates 5

Rangeland Health 6

How to use guide $\quad 6$

$\begin{array}{ll}\text { Results } & 12\end{array}$

DM-Dry Mixedwood subregion 38

A. Native grass and Shrublands $\quad 39$

Ecology of grass and shrublands $\quad 39$

Key to grasslands and shrublands $\quad 43$

Grasslands

Community types

DMA1. Sedge meadows $\quad 45$

DMA1a. Bulrush-Cattail $\quad 46$

DMA2. Marsh reedgrass meadow $\quad 47$

DMA3. Plains wormwood/Sedge $\quad 48$

DMA4. Purple oatgrass-Sedge-California oatgrass 49

DMA4a. Veiny meadow rue/Slender wheatgrass-Fringed brome $\quad 50$

DMA5. Western porcupine grass-Sedge/Fringed sage 51

DMA6. Northern wheatgrass-Junegrass/Fringed sage 52

DMA7. Saskatoon-Snowberry/Hairy wildrye 53

DMA8. Saskatoon/Sweet clover/Smooth brome 54

DMA9. Kentucky bluegrass-Hairgrass 55

Shrublands

DMA10. Willow/Sedge 56

DMA10a. Willow/Marsh reedgrass $\quad 57$

DMA11. Willow/Marsh reedgrass-Kentucky bluegrass 58

DMA12. Willow/Horsetail/Marsh reedgrass $\quad 59$

DMA13. River alder/Horsetail 60 
DMA14. Willow/Kentucky bluegrass/Dandelion 61

DMA15. Sandbar-Yellow willow 62

DMA16. Bebb willow/Marsh reedgrass 63

DMA17. Red osier dogwood/Marsh reedgrass 64

DMA18. Silverberry/Smooth brome 65

DMA19. Bog willow 66

Other riparian grasslands

DMA20. Swamp horsetail 67

DMA21. Tall manna grass 68

DMA22. Common reedgrass $\quad 69$

DMA23. Reed canary grass $\quad 70$

DMA24. Two stamened sedge $\quad 71$

DMA25. Rush 72

DMA26. Creeping spike rush $\quad 73$

DMA27. Three square rush $\quad 74$

DMA28. Prairie bulrush $\quad 75$

DMA29. Nuttall's saltgrass $\quad 76$

$\begin{array}{ll}\text { DMA30. Foxtail barley } & 77\end{array}$

$\begin{array}{ll}\text { B. Tame grasslands } & 78\end{array}$

$\begin{array}{ll}\text { Ecology of tame pastures } & 79\end{array}$

Key to the grasslands $\quad 83$

Community types

DMB12. Brome-Timothy 84

DMB13. Creeping red fescue-Brome-Timothy 85

DMB14.Creeping red fescue-Kentucky bluegrass/Dandelion 86

DMB15. Strawberry-Dandelion/Weeds $\quad 87$

DMB16. Reed canarygrass-Meadow foxtail-Brome-Timothy 88

DMB17. Brome-C. red fescue-K. bluegrass/Dandelion 89

DMB18. Foxtail barley/Weeds $\quad 90$

DMB19. Wheatgrass-C. red fescue-Timothy 91

DMB20. Rose/C. red fescue-Sedge 92

DMB21. Aw/Rose/Strawberry 93

DMB22. Rose/Dandelion/Hairy wildrye $\quad 94$

DMB23. Aw-Pb/Rose/Hairy wildrye 95

DMB24. Willow/Timothy 96

$\begin{array}{ll}\text { C. Deciduous community types } & 65\end{array}$

$\begin{array}{ll}\text { Ecology of deciduous community types } & 97\end{array}$ 
Key to deciduous community types

DMC1. Aw/Dwarf bilberry/Bearberry/Mountain ricegrass 105

DMC1a. Aw/Blueberry $\quad 106$

DMC2. Aw/Rose/Tall forb 107

DMC3. Aw/Rose/Low forb 108

DMC3a. Aw/Dandelion/Kentucky bluegrass 109

DMC4. Aw-Pb/Hazelnut 110

DMC5. Aw/Buffaloberry 111

DMC6. Aw/Alder $\quad 112$

DMC7. Aw/Saskatoon 113

DMC8. Pb-Aw/Red Osier dogwood 114

DMC8a. Pb-Aw/Willow 115

DMC9. Pb-Aw/Horsetail 116

DMC10. Deciduous cutblocks 117

DMC11. Pb/Honeysuckle 118

DMC12. Pb/River alder $\quad 119$

DMC13. Aw- $\mathrm{Pb} /$ Silverberry $\quad 120$

DMC14. $\mathrm{Pb} /$ Snowberry 121

DMC15. Pb/Reedgrass $\quad 122$

DMC16. Bw/Labrador tea $\quad 123$

DMC17. Bw/Raspberry 124

DMC18. Pb/Kentucky bluegrass 125

DMC19. $\mathrm{Pb} /$ Smooth brome 126

$\begin{array}{ll}\text { D. Mixedwood and Conifer community types } & 127\end{array}$

Ecology 128

Key to community types 130

Community types

DMD1. Pj/Alder 132

DMD2. Pj-Aw/Bearberry 133

DMB2a. Aw-Sw/Bearberry 134

DMD3. Sw/Buffaloberry/Bearberry 135

DMD4. Sw/Hazelnut/Moss 136

DMD5. Aw-Sw/Rose/Marsh reedgrass 137

DMD6. Aw-Pb-Sw/Willow/Wild sarsaparilla 138

DMD7. Sw-Pb-Aw/Rose/Twinflower 139

DMD8. Sb/Willow/Moss 140

DMD9. Sb-Lt/Labrador tea/Moss 141

DMD10. Sw-Aw/Low bush cranberry $\quad 142$

DMD11. Sw/Moss 143

DMD12. Sw-Bw/Raspberry 144 
Ecology

Key to community types

Community types

Grasslands

CMA1. Sedge meadows

CMA2. Marsh reedgrass meadow

CMA3. Cow parsnip/Kentucky bluegrass-Marsh reedgrass

CMA4. Snowberry/Kentucky bluegrass

CMA5. Plains wormwood/ Sheep fescue

175

CMA6. Plains wormwood/ Kentucky bluegrass-Sedge

Shrublands

CMA7. Willow/Sedge

CMA8. Willow/Sedge-Kentucky bluegrass

CMA9. Willow/Marsh reedgrass

CMA10. Willow-Alder/Marsh reedgrass

CMA11. Willow/Fireweed

CMA12. Willow-Spruce/Kentucky bluegrass

CMA13. Yellow willow

CMA14. Scouler willow-Red osier dogwood

184

CMA15. Bebb willow/Marsh reedgrass

Other riparian grasslands

CMA16. Swamp horsetail

CMA17. Tall manna grass

CMA18. Short sedge (boggy) 
Ecology

Key to community types

Community types

CMB1. Kentucky bluegrass/Dandelion $\quad 194$

CMB2. Kentucky bluegrass-Timothy/Dandelion $\quad 195$

CMB3. Timothy/Dandelion $\quad 196$

CMB4. Creeping red fescue-Kentucky bluegrass/Dandelion 197

CMB5. Creeping red fescue/Hairgrass 198

CMB6. Creeping red fescue-Timothy/Dandelion 199

CMB7. Smooth brome 200

$\begin{array}{ll}\text { C. Deciduous community types } & 201\end{array}$

Ecology 202

Key to community types 206

Community types

Balsam poplar dominated

CMC1. Pb/Rose-Alder 208

CMC2. Pb-Aw/River alder $\quad 209$

CMC3. Pb-Aw/Beaked hazelnut-Rose $\quad 210$

CMC3a. Aw-Pb/Honeysuckle 211

Paper birch dominated

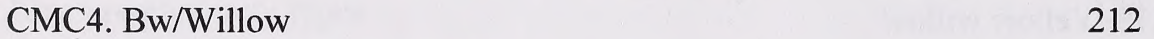

Aspen/Rose dominated

CMC5. Aw/Blueberry $\quad 213$

CMC6. Aw/Rose/Twinflower $\quad 214$

CMC7. Aw/Rose/Low forb $\quad 215$

CMC8. Aw/Rose/Tall forb $\quad 216$

CMC8a. Aw/Buffaloberry $\quad 217$

CMC9. Aw/Rose-Saskatoon 218

CMC10. Aw-Pb/Rose/Strawberry $\quad 219$

CMC11. Aw/Rose/Clover $\quad 220$

CMC12. Aw/Alder-Willow-Rose 221

CMC13. Aw/Willow 222

CMC14. Aw-Pb/Red Osier dogwood-Rose 223 
CMC15. Aw/Horsetail-Cow parsnip 224

CMC16. Aw/Smooth brome 225

D. Mixedwood and Conifer community types 226

$\begin{array}{ll}\text { Ecology } & 227\end{array}$

Key to community types 230

Community types

CMD1. Pj/Alder 231

CMD2. Pj/Bearberry 232

CMD3. Aw-Pj/Bearberry/Lichen 233

CMD4. Balsam fir-Sw/Moss 234

CMD5. Sw/Moss 235

CMD6. Sw/Creeping red fescue 236

CMD7. Aw-Sw/Rose/Low forb 237

CMD8. Aw-Sw/Labrador tea/Moss 238

CMD9. Sb/Labrador tea/Peat moss $\quad 239$

CMD10. Sb/Bog birch $\quad 240$

CMD11. Aw-Sw/Hazelnut 241

CMD12. Sw/Horsetail 242

$\begin{array}{ll}\text { Literature cited } & 243\end{array}$

\section{List of Figures}

Figure 1. Layout of the Ecoloigical Classification System for Alberta 4

Figure 2. Landscape diagram of the Ecological sites described in the Boreal Mixedwood subregions. 19

Figure 3. Edatopic grid for the Dry Mixedwood subregion 20

Figure 4. Overview of native shrub and grassland complex in the

Dry Mixedwood subregion $\quad 40$

Figure 5. Overview of deciduous communities in the Dry Mixedwood subregion 99

Figure 6. Edatopic grid for the Central Mixedwood subregion 154

Figure 7.Overview of native shrub and grassland communities in the Central

Mixedwood subregion

Figure 8. Overview of deciduous communities in the Central Mixedwood subregion

\section{List of Tables}

Table 1. Ecological sites, ecological site phases and community types of the Dry Mixedwood 
subregion $\quad 13$

Ecological site summary aa grassland $\quad 21$

Ecological site phase summary aa1 Plains wormwood $\quad 22$

Ecological site summary bb grass 23

Ecological site phase summary bb1 Western porcupine grass 24

Ecological site phase summary bb2 Northern wheatgrass 25

Ecological site phase summary d4 Saskatoon-Snowberry 26

Ecological site phase summary d1 grazed Aw 27

Ecological site phase summary d1 harvested Aw 28

Ecological site summary dd grassland $\quad 29$

Ecosite phase summary dd1california oatgrass-slender wheatgrass 30

Ecological site phase summary e4 dogwood shrubland $\quad 31$

Ecological site phase summary f4 horsetail/willow 32

Ecological site phase summary f5 horsetail/Bw 33

Ecological site phase summary g2 saline $\quad 34$

Ecological site phase summary $\mathrm{j} 3$ grassland $\quad 35$

Ecological site phase summary k2a grazed willow 36

Ecological site phase summary k3a grazed meadow 37

Table 2. Native grass and shrublands of the Dry Mixedwood subregion 41

Table 3.Tame forage community of the Dry Mixedwood subregion $\quad 81$

Table 4. Deciduous community of the Dry Mixedwood subregion 100

Table 5. Conifer and Mixedwood community of the Dry Mixedwood

subregion

129

Table 6. Ecological site, Ecological site phases and community types of the

Central Mixedwood subregion

Ecological site summary aa grass/shrubland $\quad 155$

Ecological site phase summary aal plains wormwood 156

Ecological site phase summary dla grazed Aw 157

Ecological site phase summary dlc burned Aw 158

Ecological site phase summary d3c burned grazed Sw 159

Ecological site phase summary d4 shrubland 160

Ecological site phase summary e4 shrubland 161

Ecological site phase summary k2a grazed willow $\quad 162$

Ecological site phase summary j3 grassland 163

Table 7. Native grass and shrublands of the Central Mixedwood subregion 167

Table 8. Tame forage community types for the Central Mixedwood subregion 192

Table 9. Deciduous community types for the Central Mixedwood subregion 204

Table 10. Conifer and Mixedwood community types for the Central Mixedwood subregion

\section{List of Maps}




\section{List of Photos}

Photo 1. Western porcupine grass-Sedge/Fringed sage community type 38

Photo 2. Sedge-Marsh reedgrass, willow community types 38

Photo 3. Typical range improvement clearing in the Dry Mixedwood subregion 78

Photo 4. Aw/Rose/Tall forb community type in the Dry Mixedwood subregion 97

Photo 5. Aw/Hazelnut-Rose community type in the Dry Mixedwood subregion 97

Photo 6. Pj/Bearberry community type 127

Photo 7. Plains wormwood/Sheep fescue-Sedge 164

Photo 8. Heavily grazed range improvement site 189

Photo 9. Aw/Rose/Clover community type 201

Photo 10. Balsam fir-White spruce/Moss community type 226 


\begin{abstract}
The Dry and Central Mixedwood subregions cover nearly $40 \%$ of the province and are dominated by aspen, jack pine on coarse textured soils and black spruce, willows and sedges in the poorly drained areas. The vegetative communities in these subregions are important because they provide summer range for livestock, prime habitat for many species of wildlife, productive watersheds, recreational areas and timber harvesting. Despite the importance of these vegetation types there is little information on their ecology. The lack of information makes it very difficult to develop sustainable management prescriptions for multiple use. As a result guides like this and "Ecosites of Northern Alberta" (Beckingham and Archibald 1996) are being developed to provide a framework that will easily group the vegetative community types. It is hoped these classification systems can be used by field staff to assess the ecology of the sites and develop management prescriptions on lands within each region.

This guide represents the analysis of 895 grass, shrubland, conifer and deciduous plots described in the Dry and Central Mixedwood subregions. These types are split into:
\end{abstract}

\title{
Dry Mixedwood subregion
}
A. Native grasslands and shrubland
B. Tame forage communities
33 types
C. Deciduous community types
13 types
D. Mixedwood and Conifer community types
21 types
14 types

\section{Central Mixedwood subregion}
A. Native grassland and shrubland
18 types
B. Tame forage communities
7 types
C. Deciduous community types
18 types
D. Mixedwood and Conifer community types
12 types 


\section{Introduction}

The province of Alberta is covered by a broad spectrum of vegetation regions from prairie in the South, to alpine vegetation in the mountains and dense forests in the Central and Northern parts of the province. These broad vegetation regions have been classified into 6 regions and 20 subregions (Dept. of Environmental Protection 1994). Within each subregion, there are groups of plant communities which exist under similar, localized, environmental conditions and can be further influenced by human impacts. Sustainable management of these subregions requires an understand the ecology of the site coupled with the ability to recognize the vegetative communities that have similar productivity and response to disturbance.

Vegetative communities in the province of Alberta are highly regarded by most resource managers for their ability to provide a wide variety of benefits. They are a classic example of multiple use land, providing summer range for livestock, prime habitat for many species of wildlife, productive watersheds and recreational areas. Despite the importance of these vegetation types there is little information on their ecology. The lack of information makes it very difficult to development sustainable management prescriptions for multiple use.

The purpose of this guide was to develop a framework that would easily group the plant community types utilized by livestock in the Dry and Central Mixedwood subregions of the province. Plant communities are grouped into a hierarchal system based on ecology. These groupings include successional communities which occur under natural succession, or disturbance such as fire, timber or grazing operations. All of the known relationships among communities are described within this guide in table format and/or schematically. Additionally, each known plant community is described in detail.

It is hoped this classification system can be used by field staff to assess the ecology and sustainable stocking rate of sites in order to develop management prescriptions on lands within each subregion. This guide supplements the work done by Beckingham and Archibald (1996) on the forested community types in the Boreal Mixedwood of northern Alberta. Their guide is a good description of the forested community types found within the subregions, but it does not include forage production values or grazing management information. It also does not provide a description of the native grassland and shrubland communities which are utilized extensively by livestock in these subregions.

\section{Climate and Modal Plant Communities}

\section{Dry Mixedwood subregion}

The Dry Mixedwood (DM) subregion represents a transition between the Central and Peace River Parklands and the Central Mixedwood subregions. This subregion occurs in three areas of the province. One section is located between the Central Parkland and the Central Mixedwood subregions in the southern portion of the boreal forest and includes the Onion Lake, Athabasca, Westlock plains and Whitefish and Frog Uplands ecodistricts (Strong and Thompson 
1995). A second area is located immediately east of Edmonton in the Cooking Lake upland ecodistrict. The third and largest area parallels the Peace River in northwestern Alberta from Grande Prairie to Fort Vermillion and includes the Debolt, Dunvegan, Falher, Smoky, Grimshaw, Manning, High Level and Boyer plains ecodistricts (Map 1).

Mean summer temperature averages $13.8^{\circ} \mathrm{C}$ and winter temperatures average $-10.5^{\circ} \mathrm{C}$, which is somewhat warmer than the Central Mixedwood subregion and somewhat cooler than the Parkland subregions. Mean annual precipitation averages $380 \mathrm{~mm}$ which is drier than the Central Mixedwood, but wetter than the Parkland subregions.

The modal plant community in this subregion is dominated by Aspen, with a variable understory dominated by rose, pea-vine, beaked hazelnut, saskatoon and marsh reedgrass. Jack pine stands are found on well drained, coarse-textured parent materials and poorly drained sites are dominated by black spruce, willows and sedge species.

\section{Central Mixedwood subregion}

The Central Mixedwood (CM) subregion is the largest in the province covering over $210,000 \mathrm{~km}^{2}$ or nearly $32 \%$ of the province (Strong and Leggat 1992)( Map 1). Mean annual summer temperatures average $13.5^{\circ} \mathrm{C}$ and winter temperatures average $-13^{\circ} \mathrm{C}$, which is somewhat colder than the adjacent Dry Mixedwood subregion. Annual precipitation averages $397 \mathrm{~mm}$ of precipitation which is wetter than the Dry Mixedwood.

The modal plant communities are vegetated by aspen and balsam poplar with understories composed of a variety of herbs and deciduous shrubs. White spruce and balsam fir are the climatic climax species but are not well represented because of the frequent occurrence of fire. On dry, well drained, coarse-textured soils jack pine dominates and the poorly drained sites are dominated by black spruce, willows and sedge species. These communities are very similar to the Dry Mixedwood subregion, but drier conditions of the Dry Mixedwood favours formation of a number of native grassland communities which are not found in the Central Mixedwood.

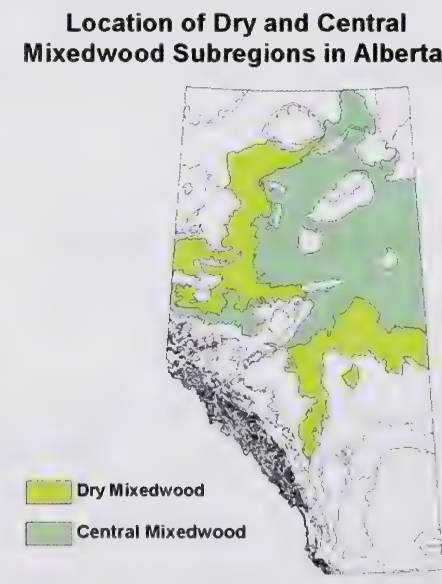




\section{Approach and Methods of Classification}

\section{Approach: Ecological classification hierarchy and terminology}

The system of classification in this guide was initially based on the community type approach of Mueggler (1988). Mueggler's system was chosen over the habitat type approach (Daubenmire 1952) or ecosystem association approach (Corns and Annas 1986) because it could classify plant communities irregardless of their successional status. However, as the philosophy of rangeland health and proper functioning condition of a site evolved, it became apparent (through data analysis) that there was a need to also organize the various plant communities based on their response to disturbance (i.e. disturbance vs. natural succession) within an area under similar environmental influences.

It was determined that the ecosystem classification system developed by Corns and Annas (1986) and Beckingham et al. (1996) could accommodate this additional requirement. Thus, the new system developed for rangelands is a combination of Mueggler (1988) and Beckingham et al. (1996). Consequently, this guide adopts a similar ecological unit classification hierarchy (ecosite, ecosite phase, plant community). In an effort to first, link the hierarchical system with the historic rangeland system, and second, to create a provincially standardized rangeland approach, slightly different classification terminology was developed. The new terms ecological site and ecological site phase (replacing Beckingham et al.'s [1996] ecosite and ecosite phase terms respectively), provide subtle distinction to recognize the blending of the old systems and still be recognizable to readers familiar with the original terminology. See figure 1 for a flow chart of both classification and general presentation of information.

\section{Methods: Plant community classification}

Sampling for this guide occurred within the Dry and Central Mixedwood subregions . This guide outlines the classification of 685 plots described in the Dry Mixedwood and 210 plots described in the Central Mixedwood subregions.

The procedure for inventory of plots followed the Range Survey Manual (1992) and uses the MF5 form. A plot consisted of a $10 \mathrm{~m} \times 10 \mathrm{~m}$ macroplot and ten randomly selected $1 \mathrm{~m} \mathrm{x} 1$ $\mathrm{m}$ microplots to record the canopy cover of shrubs and ten nested $20 \mathrm{~cm} \times 50 \mathrm{~cm}$ microplots to record the canopy cover of forbs and grass. For a description of the methodology for riparian plots done in the Mixedwood subregions see (Thompson and Hansen 2004). The data for each site was analyzed using the multivariate analysis techniques of classification and ordination. Classification is the assignment of samples to classes or groups based on the similarity of species. A polythetic agglomerative approach was used to group the samples. This technique assigns each sample to a cluster which has a single measure. It then agglomerates these clusters into a hierarchy of larger and larger clusters until finally a single cluster contains all the samples (Gauch 1982). Cluster analysis was performed in SAS and Euclidean distance was used as the 


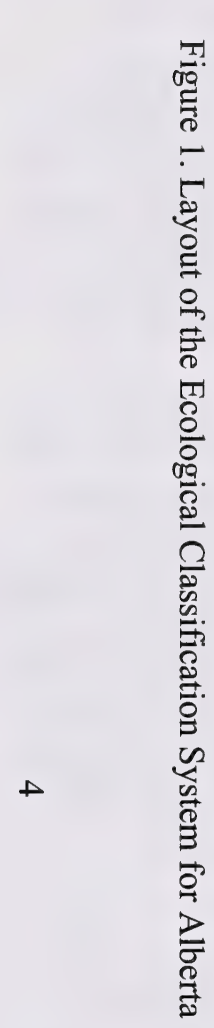

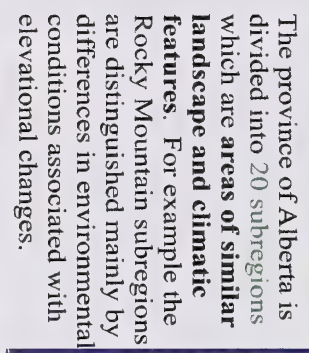

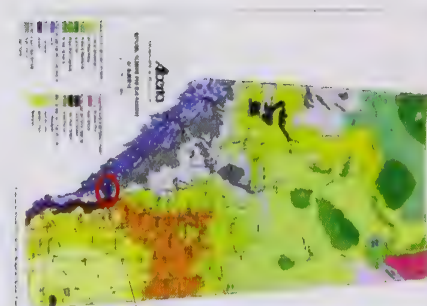

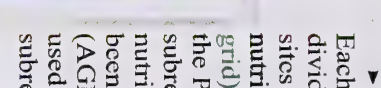
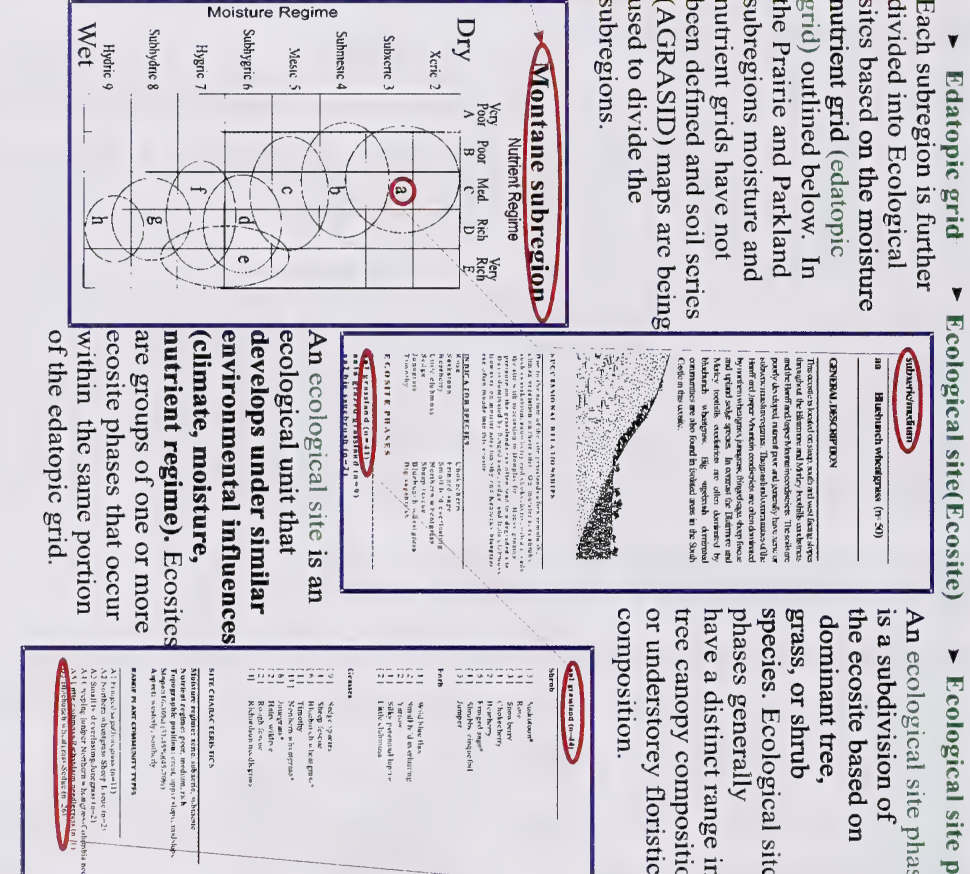

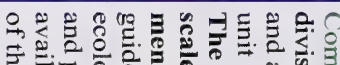

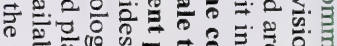

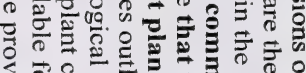

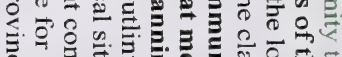

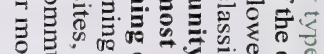

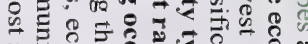

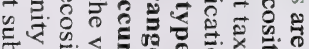
牙节产. og.

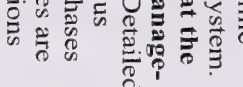

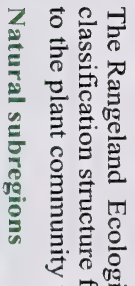

स

?

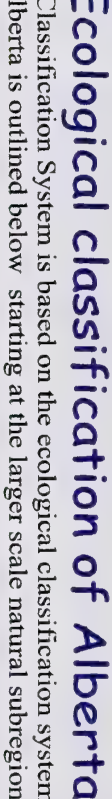

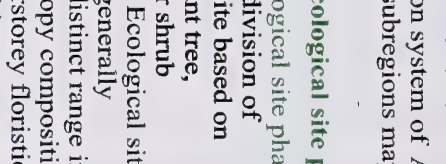

농

突. 길.

웅.

3

ำ

ถ 
Cluster Distance Measure and Ward's method was used in the Group Linkage Method. The groupings generated in cluster analysis were overlain on the site ordination to determine final groupings. Ordination was used to find relationships among species, communities and environmental variables. Ordination reduces the dimensionality of the data to 1-3 most important axes to which environmental gradients can be assigned. The ordination technique used in the analysis of the data was DECORANA (Detrended Correspondence Analysis).

DECORANA detrends and rescales the axes thereby reducing the arching and compression of axes problems associated with other ordination techniques (Reciprocal averaging, Principle Components Analysis). Once final groupings were determined on the ordination specific environmental variables can be assigned to the variation outlined on the ordination axes.

Plant community type summaries were generated in SAS, by averaging plant species composition, range in composition, and percent constancy of occurrence, among vegetation inventory plots which were part of a community type. Environmental data was subsequently sorted into the same plant community groupings to create the plant community descriptions outlined in this guide. The number of sample plots on which the description was based is also provided (e.g. $\mathrm{n}=16$ ).

\section{Range Management Concepts and Methods}

\section{Ecologically sustainable stocking rates}

Ecologically sustainable stocking rates (ESSR) values are suggested for each plant community. These values reflect the maximum number of livestock (e.g. hectares(ha)/animal unit month(AUM)) that can be supported by the plant community given inherent biophysical constraints and the ecological goal of sustainable health and proper functioning of the plant community. When the ESSR is multiplied by the area (e.g. ha) of a plant community polygon the result is termed ecologically sustainable carrying capacity (ESCC), and is expressed as AUMs. Often the ESCC must be adjusted for management factors (e.g. reduced livestock distribution), management goals (e.g. improve rangeland health, multiple use and values, etc.), drought conditions, and other natural phenomena impacting the site (e.g. forage quality, fire, pests, etc.). This adjusted/reduced value is the ecologically sustainable grazing capacity

(ESGC). The ESGC values are not provided in the plant community guide because the necessary adjustments are determined by the rangeland resource manager.

Suggested ESSR values were determined from a combination of clipping studies, longterm rangeland reference area data, estimated production, and historical grazing experience. In order to sustain ecological health and function of the plant community, the ESSR was based on the allocation of $25 \%$ of total production for forested plant community types, $50 \%$ of total production for grass and shrub land types within the Dry and Central Mixedwood subregions and the forage requirements one animal unit (i.e. $455 \mathrm{~kg}$ of dry matter per month). The remaining biomass production (carry over), is allocated for the maintenance of ecological functions (e.g. nutrient cycling, viable diverse plant communities, hydrological function, and soil protection, etc.) and plant community services (forage production, habitat maintenance, etc.). The allocation of biomass production in this manor is well established, and supported, by the scientific 
community and the amount required, varies with Natural Subregion (Holechek et al. 1995).

\section{Rangeland Health}

Range health is determined by comparing the functioning of ecological processes on an area (e.g. plant community polygon) of rangeland to a standard (i.e. RPC) described within an ecological site description. An ecological site is similar to the concept of range site, but a broader list of characteristics are described. An ecological site is defined by the Task Group on Unity and Concepts (1995) as, "a distinctive kind of land with specific physical characteristics that differs from other kinds of land in its ability to produce a distinctive kind and amount of vegetation". This guide can be used to determine the appropriate reference range plant community, within an ecological site, for a rangeland health assessment.

Rangeland health assessments are utilized to make a rapid determination of the ecological status of rangeland. We use range health terminology (healthy, healthy with problems, or unhealthy), to rank the ability of rangeland to perform certain ecological functions. These functions include: net primary production, maintenance of soil/site stability, capture and beneficial release of water, nutrient and energy cycling and plant species functional diversity. For a detailed description on how to assess rangeland health for various plant communities please refer to "Rangeland Health Assessment for Grassland, Forest and Tame Pasture" (Adams et al. 2003). A general range health category (Healthy, Healthy with problems, Unhealthy) has been added to each community type description, which can be used as a guide when doing range health assessments.

Range management objectives tend to favor the later stages of plant succession (lateseral to potential natural community (PNC) or good to excellent range condition) (Adams et al. 2003). Late seral plant communities tend to be superior in the efficient capture of solar energy, in cycling of organic matter and nutrients, in retaining moisture, in supporting wildlife habitat values and in providing the highest potential productivity for the site. In contrast, early seral stages represent plant communities with diminished ecological processes, which are less stable and more vulnerable to erosion and invasion by weeds and non-native species. They also have diminished resource values for livestock forage production, wildlife habitat and watershed protection (Adams et al. 2003). Healthy rangelands perform important ecological functions and provide a broader suite of goods and services. In most cases these late seral plant communities are used as reference range plant community (RPC), but sometimes management goals influence the choice of RPC (e.g. a cut block to be maintained as untimbered rangeland).

\section{How to use the guide}

\section{Organization of the guide}

This guide is an expansion of the Ecosites of Northern Alberta guide (Beckingham and Archibald 1996). It contains new information and it is recommended that the reader has access 
to relevant information from both guides. The community types in this guide are closely related to the ecosites and ecosite phases outlined in Ecosites of Northern Alberta (Beckingham and Archibald 1996), and are similarly arranged (e.g. Table 1). Table 1 and Table 6 are a reproduction of Figure 11 in Ecosites of Northern Alberta with community types in this guide further separated into reference range plant communities, successional communities and harvesting and fire communities. The "Successional community types" or "Harvesting and Fire succession" categories outline the successional sequence the community types undergo with heavy grazing pressure, harvesting or fire disturbance.

The majority of ecological site and ecological site phase summary tables as well as the plant community descriptions are recorded in Ecosites of Northern Alberta (Beckingham and Archibald 1996). Any new ecological sites and ecological site phases reported in this guide are summarized before the community type descriptions. The bulk of this guide is community descriptions which include information on the dominant plant species, canopy cover, environmental conditions, response to grazing, forage production and suggested ESSRs. When available, we have included plant community successional information to help us determine rangeland health and the successional relationships on an ecological site.

Generally, in both guides, ecological units within a subregion are classified by their position on the edatopic grid [a specific combination of soil moisture and soil nutrient regime] (Figures 3 and 6).

The information in this guide is presented and named by:

1. Subregion/Ecological area

a. Dry Mixedwood [DM]

b. Central Mixedwood [CM]

2. Dominant cover type

a. Native grasslands and Shrublands [A]

b. Tame forage communities [B]

c. Deciduous forest [C]

d. Mixedwood and Conifer forest [D]

NOTE: Each dominant cover type may overlay several ecological sites and ecological site phases. For example DMA community types occur in 8 ecological sites [aa, bb, c, d, dd, f, k, and 1$]$.

3. Community types are presented and named by:

a. Subregion/Ecological area and dominant cover type [e.g. DMA].

b. Position on the edatopic grid. Generally, communities are named/numbered from low moisture /nutrient status to high moisture/nutrient status. For example, DMA3 is a Plains wormwood/Sedge community on the"aa" xeric/poor ecological site, while DMA7 is a Saskatoon-snowberry/hairy wild rye community type on the "d" mesic medium ecological site.

NOTE: As additional information is collected and new ecological units are identified and described, an attempt is made to fit them into the pre-existing ones. At times the usual conventions of naming and organization have to be compromised to accommodate the new units. Sometimes it was necessary to add an additional letter to an existing name to wedge 
the new unit into the appropriate place within the pre-existing ones. For example, the extra letter in the new ecological site "dd" and the pre-existing ecological site " $d$ ".

\section{New information presented}

For the most part ecological sites and ecological site phases are the same in both this and the Ecosites of Northern Alberta guide (Beckingham and Archibald 1996), particularly for the forested community types. Analysis of plot data identified a number of new ecological sites for the grass and shrubland community types which were then described.

1. Dry Mixedwood (Table 1)

a. New ecological sites and New ecological site phases

i (aa)(xeric/poor) grassland

1. (aa1) Plains wormwood grassland

ii (bb)(subxeric/medium) grassland

1. (bb1) Western porcupine grass grassland

2. (bb2) Northern wheat grass grassland

iii (dd)(mesic/rich) grassland

1. (dd1) California oatgrass-slender wheat grass grassland

b. New ecological site phases (successional) within pre-existing ecological sites

1. (dla) grazed Aspen

2. (d1b) harvested Aspen

3. (d4) Shrubland

4. (e4) dogwood shrubland

5. (f4) horsetail/willow

6. (f5) horsetail/White birch

7. (g2) Saline

8. (j3) grassland poor fen

9. (k2a) grazed willow

10. (k3a) grazed meadow

\section{Central Mixedwood (Table 6)}

a. New ecological sites and New ecological site phases

i (aa)(xeric/poor) grassland

1. (aa1) Plains wormwood grassland

b. New ecological site phases within pre-existing ecological sites

1. (d4) Shrubland

2. (d1a) grazed Aspen

3. (d1c) burned Aspen

4. (d3c) burned White Spruce

5. (e4) dogwood shrubland

6. (j3) grassland poor fen

7. (k2a) grazed willow 


\section{Identifying plant community types}

There are two methods to identify plant community types in this guide. The first method uses a key within the dominant cover categories of native grass and shrubland, tame forage. deciduous, or mixedwood and conifer. The second method involves using soil moisture and nutrient information and indicator species to identify plant community types.

\section{Method 1. Use dichotomous key within dominant cover categories}

Step 1. Pick the appropriate subregion [DRY MIXEDWOOD or CENTRAL MIXEDWOOD].

Step 2. Pick the appropriate category the community type is in within each subregion.

A. The area does not have an overstory tree canopy and has not been cleared and broken, the community will fall under the NATIVE GRASSLANDS and SHRUBLANDS category.

B. The area has been cleared of trees, broken, and seeded down to tame forage species such as timothy or creeping red fescue, the community will be in the TAME GRASS category.

C. The DECIDUOUS category includes all plant communities that are dominated by deciduous tree species. Deciduous cutblocks are included here.

D. Communities which have begun to undergo succession from a deciduous to a conifer dominated overstory with an overstory cover greater than $15 \%$ for both deciduous and coniferous species fall into the MIXEDWOOD category. Those communities dominated by a conifer overstory, White spruce, Black spruce or Jack pine are classified in the CONIFER category.

Step 3. Turn to the appropriate section [e.g. DMA] and work through the key provided to determine the community type of the site you are evaluating.

Step 4. This step is necessary only if you are completing a rangeland health assessment. In order to determine the health status of the site in question, you must decide the appropriate reference range plant community $[\mathbf{R P C}]$ to compare it to. Depending on the type of disturbance [grazing, timber operations, etc.] successional pathways may differ. The RPC would usually be the plant community that is at the start of the pathway. Management goals can influence the choice of RPC. For example, if an Aspen rose community on a "d" ecological site [e.g. DMC2] had undergone timber harvest, had not been seeded with tame forage species and the goal was to maintain it as a native community with out a mature Aspen canopy, the appropriate RPC would be DMC10. Alternatively, if the site was to be cultivated, seeded and managed as a tame pasture, the appropriate RPC might be DMB12. 


\section{Method 2. Use edatope and indicator species}

Step 1. Pick the appropriate subregion [DRY MIXEDWOOD or CENTRAL MIXEDWOOD]. [e.g. DM]

Step 2. Determine the appropriate ecological site based on position on the edatopic grid for the subregion. First decide soil moisture status, then soil nutrient status of the site in question. Use any available soils information to assist [e.g. AGRASID, or PLC]. [e.g. DM - mesic/medium is the " $\mathrm{d}$ " low-bush cranberry ecological site or DM-d]

Step 3. Look up the possible ecological site phases within the selected ecological site on Table 1 or 6. [e.g. DM-d has "d1" low-bush cranberry Aspen; "d2" low-bush cranberry Aspen-White spruce; "d3"low-bush cranberry White spruce; and "d4" shrubland.]

Step 4. Select the appropriate ecological site phase by first determining the dominant overstory [i.e the highest layer of vegetation which can be either a tree, shrub, or grass species]. [e.g. For a site dominated by Aspen (i.e. DM-d1), the appropriate ecological site phase is " $\mathrm{d} 1$ " low-bush cranberry Aspen.]

Step 5. Select the appropriate community type. Within the selected ecological site phase, use indicator understory species to choose the closest matching community type. This information is shown in table 1 or 6 as part of the community type name [e.g. DMC7 Aspen/Saskatoon]. It is also detailed in the specific community type descriptions [i.e. species with the highest average canopy cover and consistency]. At times, the community in question does not seem to match any of the known/reported types. When this happens, consider the following information in the detailed community type descriptions.

1. In the general description text.

a. The number of plots utilized to describe the community [n=number of plots]. The greater the number of plots [i.e. information available], the greater the level of confidence in the clarity and accuracy of the description including the suggested ESSR.

b. Information about where the community is found on the landscape and response to disturbance and natural succession. Use this information together with your field experience to determine the likely hood of a similar situation occurring on the site in question.

2. Under Plant Composition heading.

a. The range of a plant species canopy cover. For example, a species with a range of $0-25 \%$ may not always be visible on the site, having $0 \%$ canopy cover or it may have up to $25 \%$ cover.

b. The consistency value. This indicates the percentage of the plots that the species was actually present. So if $n=16$ and consistency was $75 \%$, then the species occurred in 12 of the plots and not in 4 of them.

Step 6. This step is the same as step 4 in method 1 and is necessary only if you are completing a rangeland health assessment. In order to determine the health status 
of the site in question, you must decide the appropriate reference range plant community [RPC] to compare it to. Depending on the type of disturbance [grazing, timber operations, etc.] successional pathways may differ. The RPC would usually be the plant community that is at the start of the pathway. Management goals can influence the choice of RPC. For example, if an Aspen rose community on a "d" ecological site [e.g. DMC2] had undergone timber harvest, had not been seeded with tame forage species and the goal was to maintain it as a native community with out a mature Aspen canopy, the appropriate RPC would be DMC10. Alternatively, if the site was to be cultivated, seeded and managed as a tame pasture, the appropriate RPC might be DMB12. 


\section{Results}

This guide represents the analysis of 895 grass, shrubland, conifer and deciduous plots described in the Dry and Central Mixedwood subregions. These types are split into:

\section{Dry Mixedwood subregion}
A. Native grasslands and shrubland
33 types
B. Tame forage communities
13 types
C. Deciduous community types
21 types
D. Mixedwood and Conifer community types
14 types

\section{Central Mixedwood subregion}
A. Native grassland and shrubland
18 types
B. Tame forage communities
7 types
C. Deciduous community types
18 types
D. Mixedwood and Conifer community types
12 types

The dominant plant species, canopy cover, environmental conditions, forage production and suggested stocking rate are outlined for each community type. 


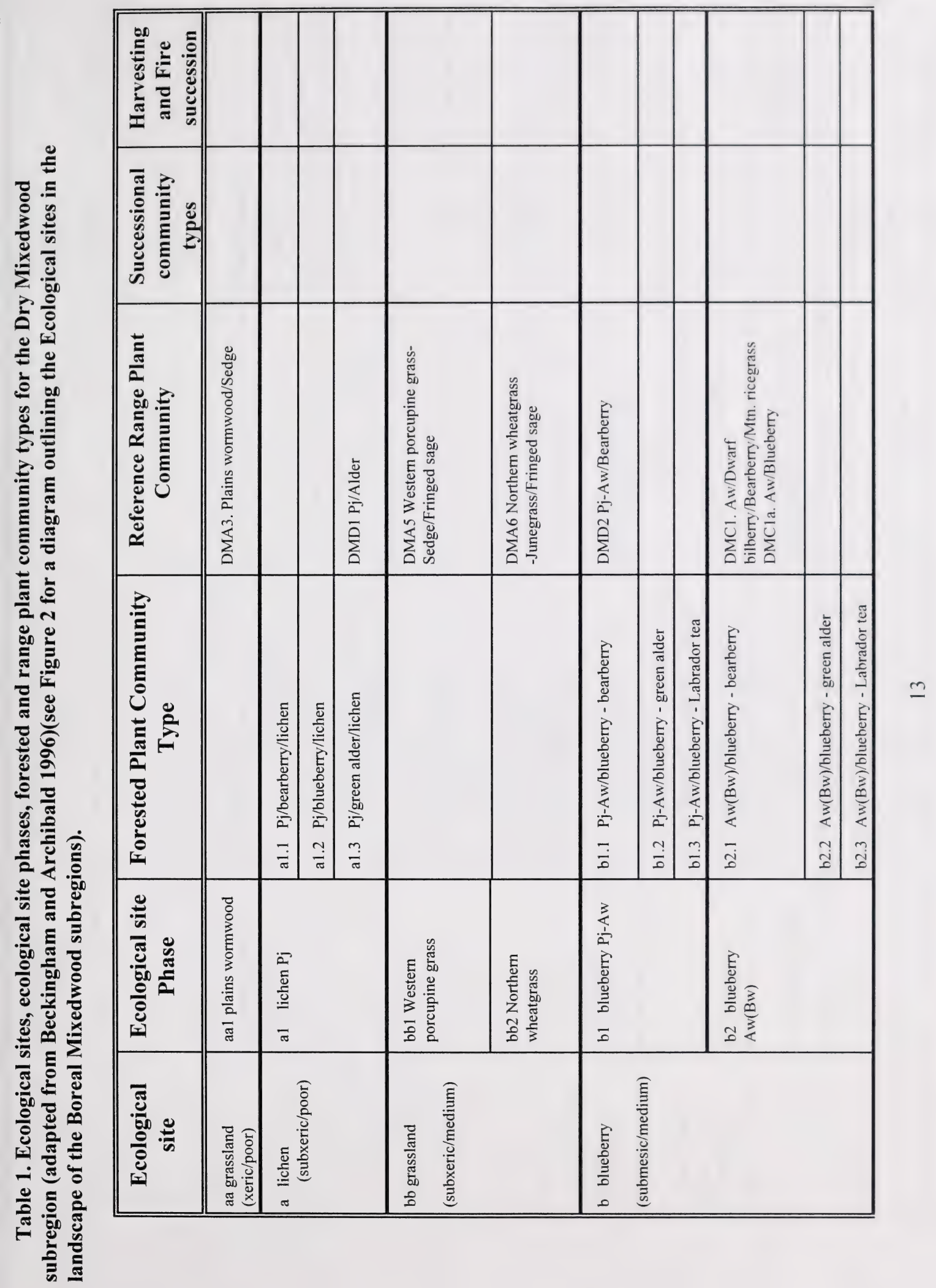




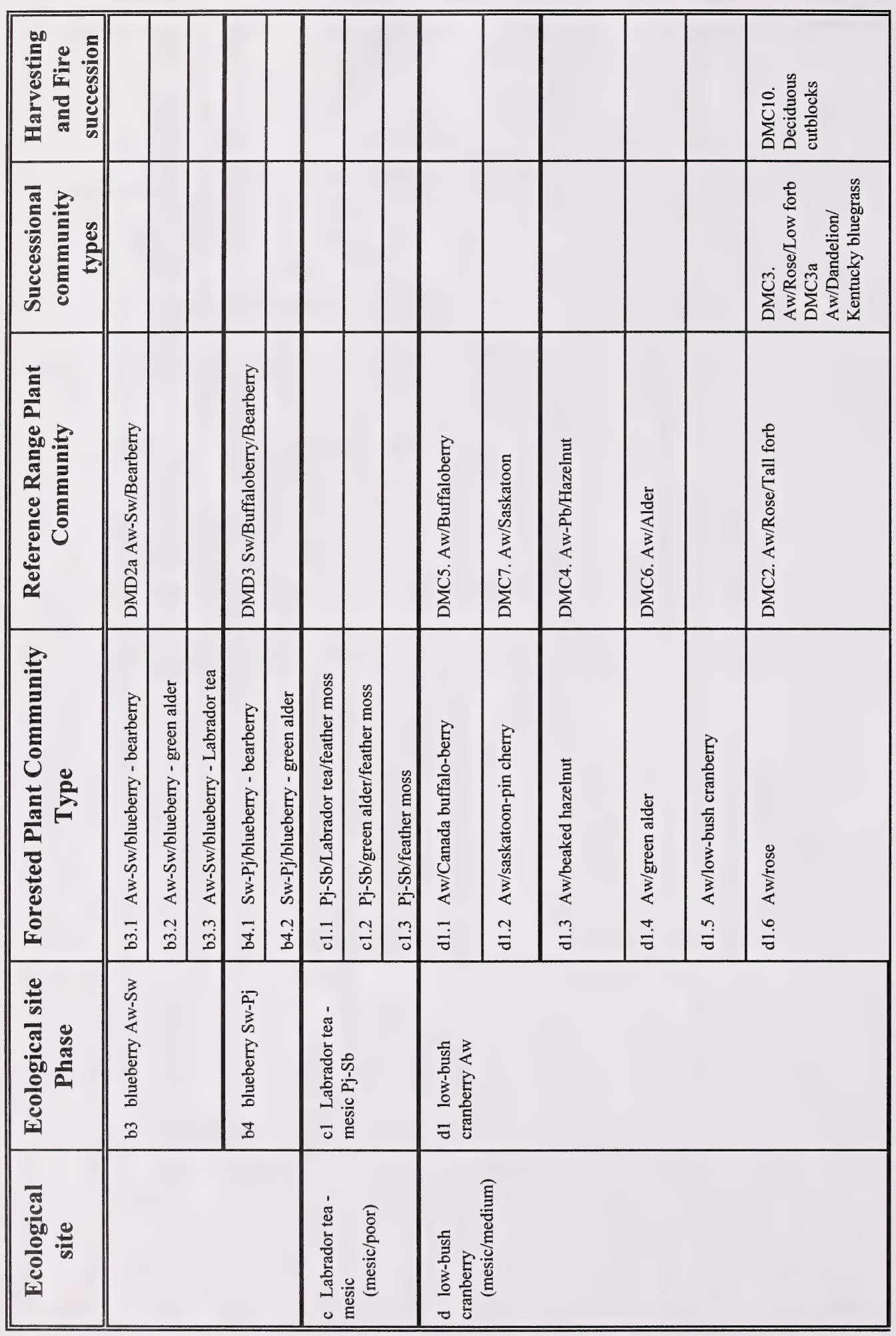




\begin{tabular}{|c|c|c|c|c|c|c|c|c|c|c|c|c|c|c|c|c|c|}
\hline 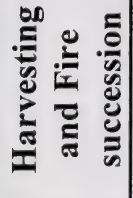 & & & & & & & & & & & & & & & & 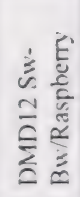 & \\
\hline 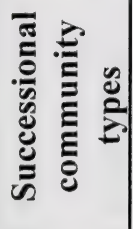 & & & & & & & & & & & & & & & & & 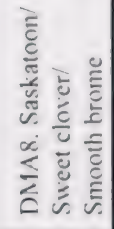 \\
\hline 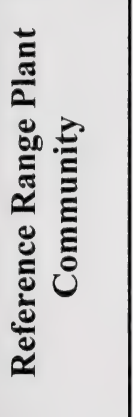 & $\begin{array}{l}0 \\
0 \\
0 \\
0 \\
0 \\
0 \\
0 \\
0 \\
0 \\
0 \\
0 \\
0 \\
0\end{array}$ & & & & & & 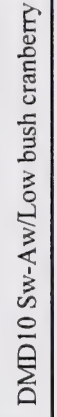 & 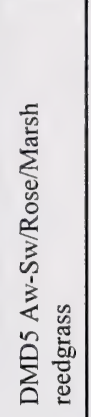 & & & & & 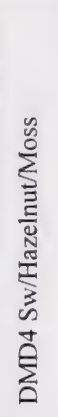 & 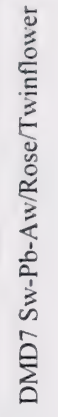 & & 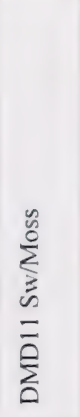 & 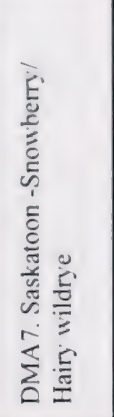 \\
\hline 氞 & 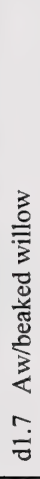 & 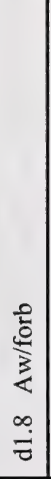 & 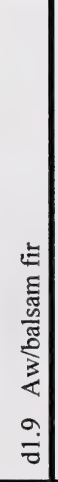 & 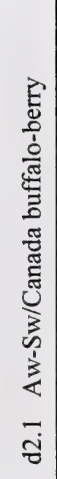 & 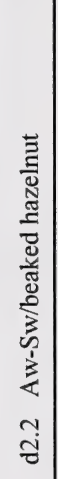 & 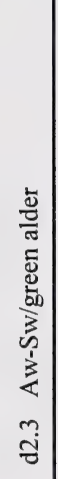 & 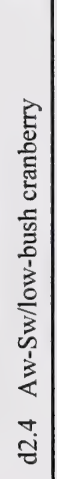 & 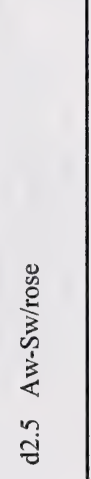 & 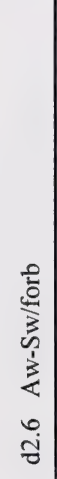 & 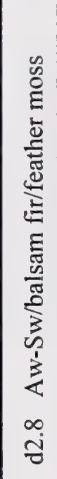 & 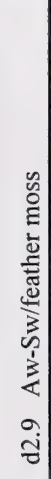 & 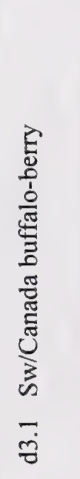 & 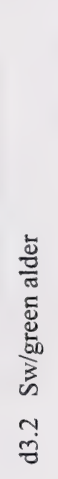 & 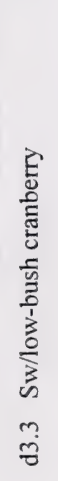 & 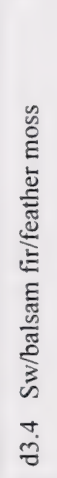 & 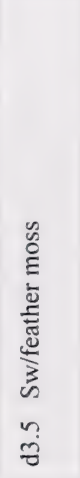 & \\
\hline 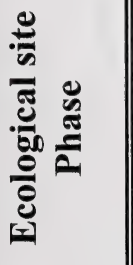 & & & & 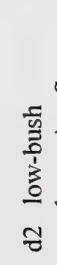 & & & & & & & & 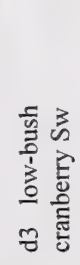 & & & & & 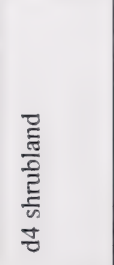 \\
\hline 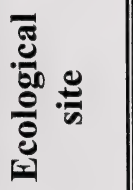 & & & & & & & & & & & & & & & & & \\
\hline
\end{tabular}




\begin{tabular}{|c|c|c|c|c|c|c|c|c|c|c|c|c|c|c|}
\hline 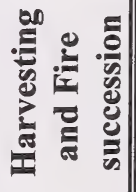 & & & & & & & & & & & & & & \\
\hline 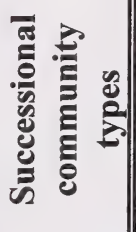 & & 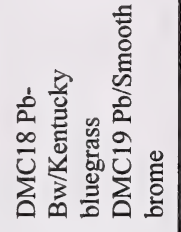 & & & & & & & & & & & & 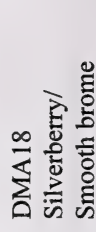 \\
\hline 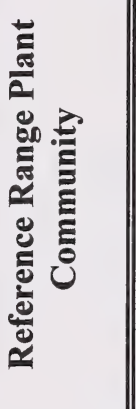 & 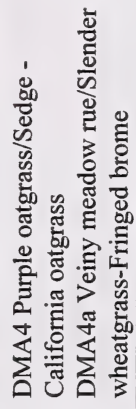 & 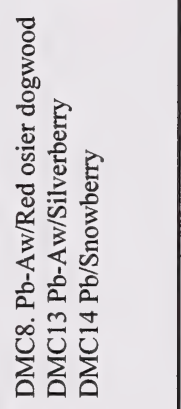 & 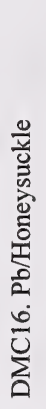 & 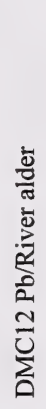 & 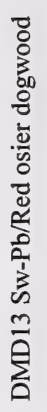 & 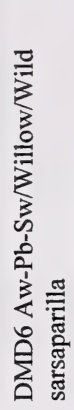 & & & & & & & & 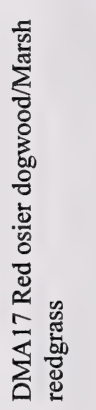 \\
\hline 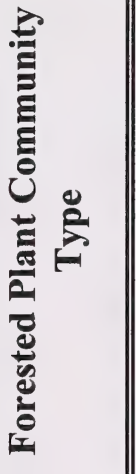 & & 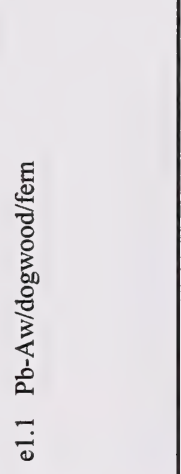 & 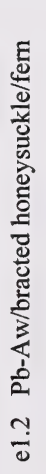 & 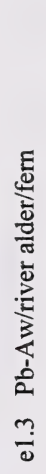 & 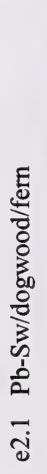 & 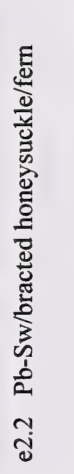 & 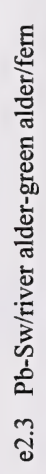 & 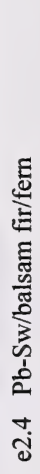 & 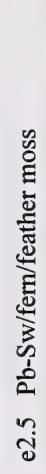 & 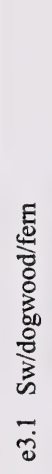 & 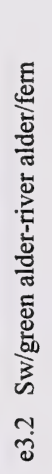 & 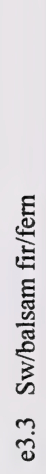 & 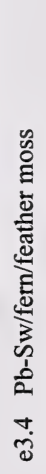 & \\
\hline 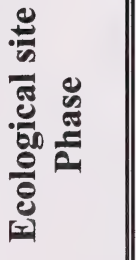 & 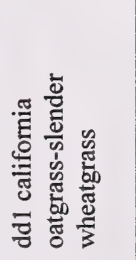 & $\begin{array}{l}3 \\
\sum_{1}^{3} \\
0 \\
0 \\
0 \\
0 \\
0 \\
00 \\
0 \\
0 \\
0\end{array}$ & & & $\begin{array}{l}3 \\
0 \\
0 \\
0 \\
0 \\
0 \\
0 \\
0 \\
0 \\
0 \\
0 \\
0\end{array}$ & & & & & 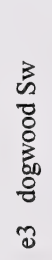 & & & & 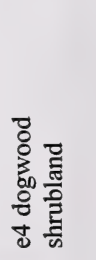 \\
\hline 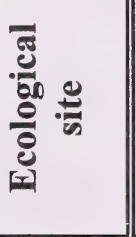 & 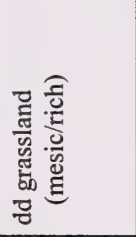 & 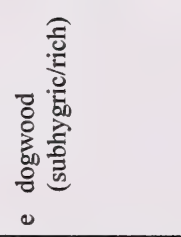 & & & & & & & & & & & & \\
\hline
\end{tabular}




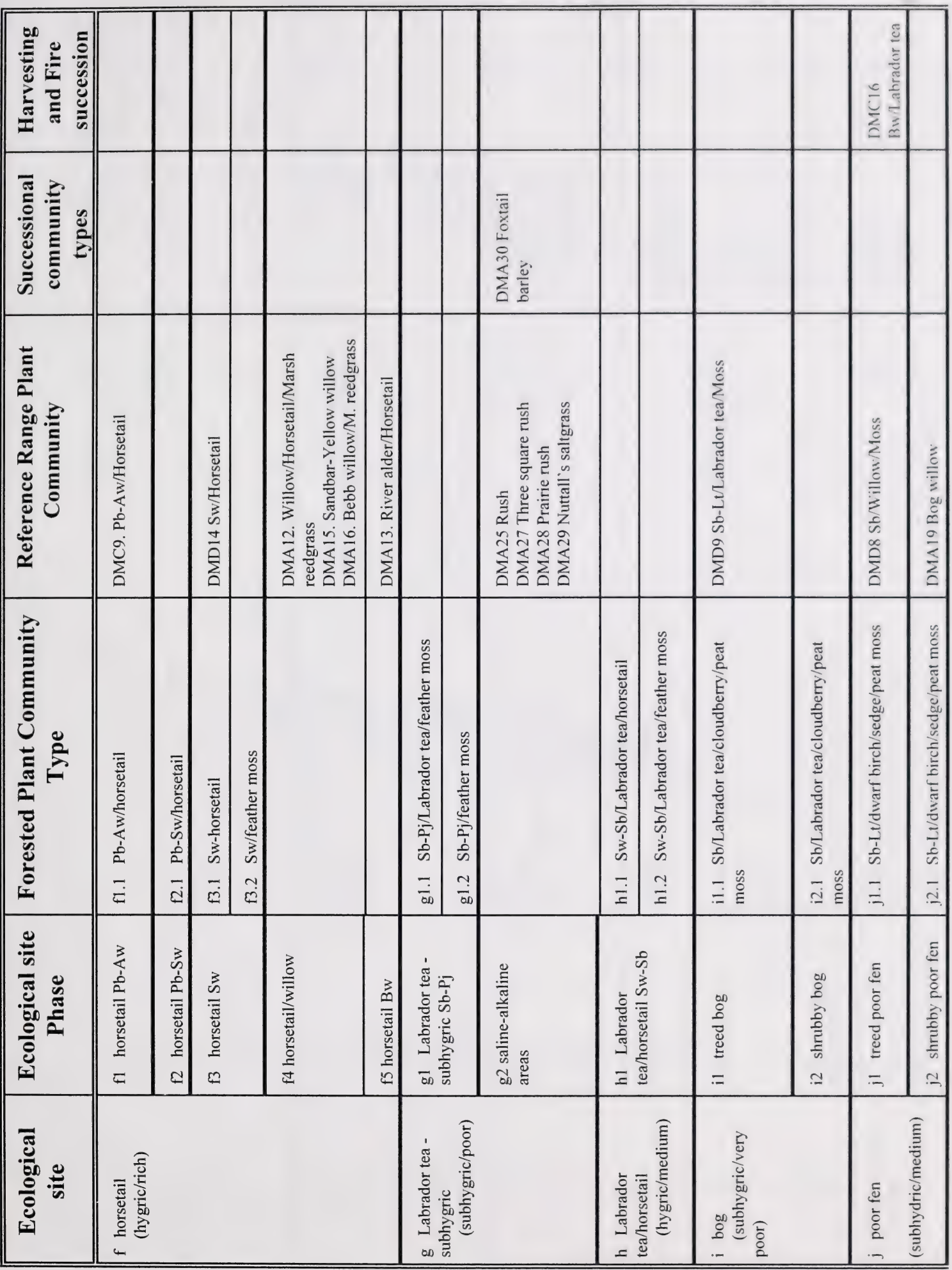




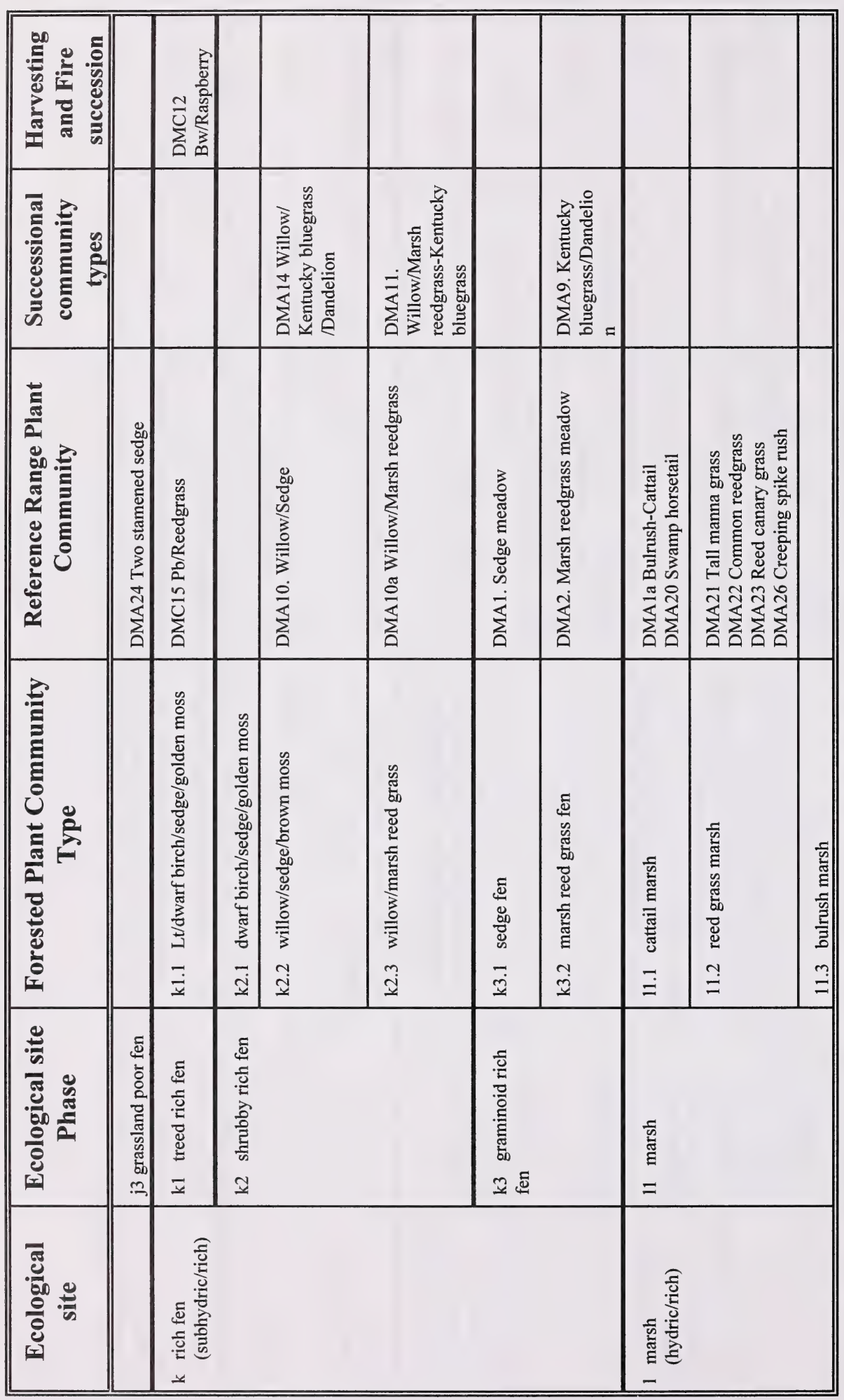



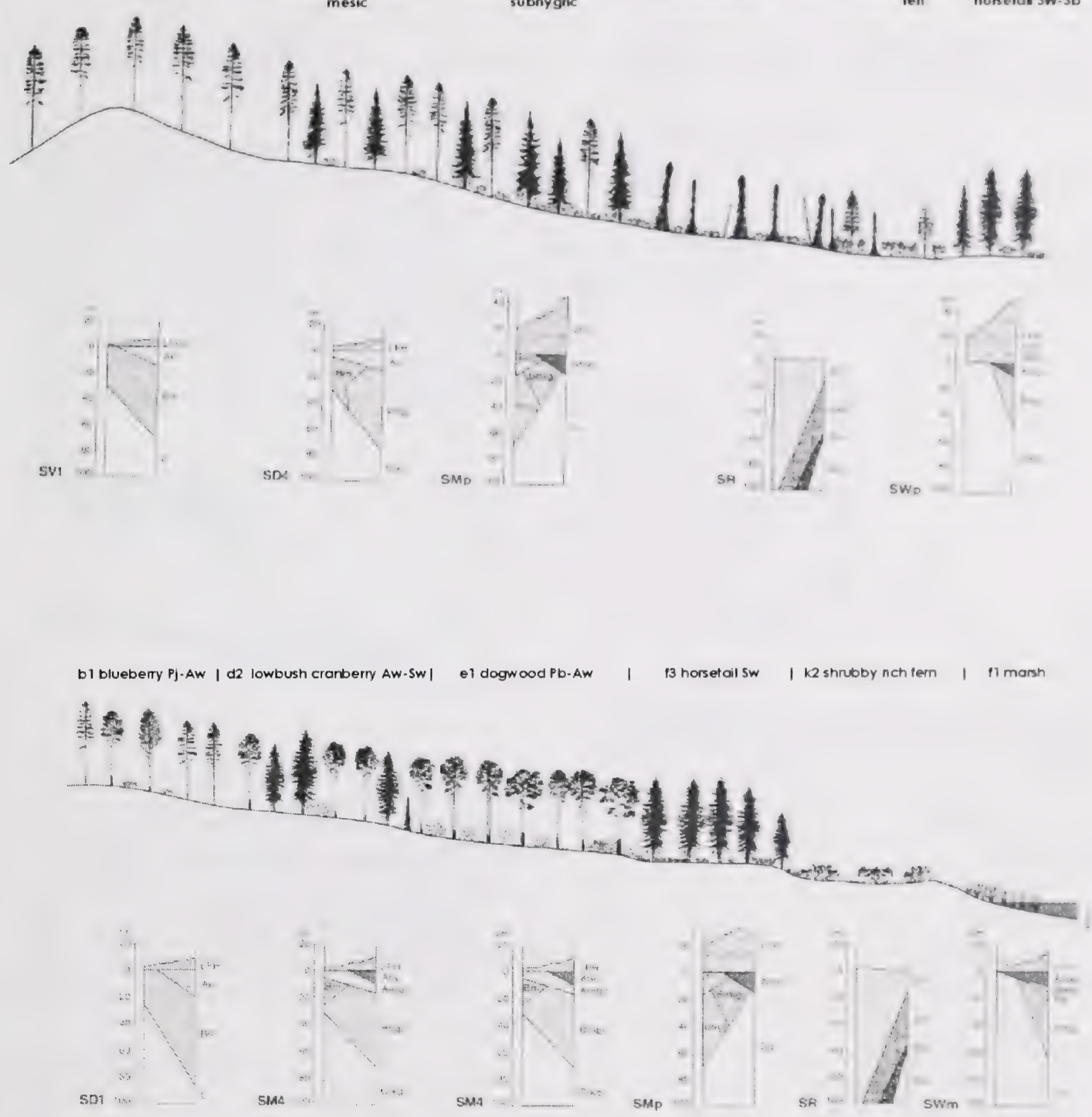

Source: Figure 12 Schematic cross section of the Boreal Mixedwood

represented by common ecosite phases and soil types. (Beckingham and Archibald 1996)

Figure 2. Landscape diagram of the Ecological sites described in the Boreal Mixedwood subregions. 
Figure 3. Edatopic grid for the Dry

Mixedwood subregion

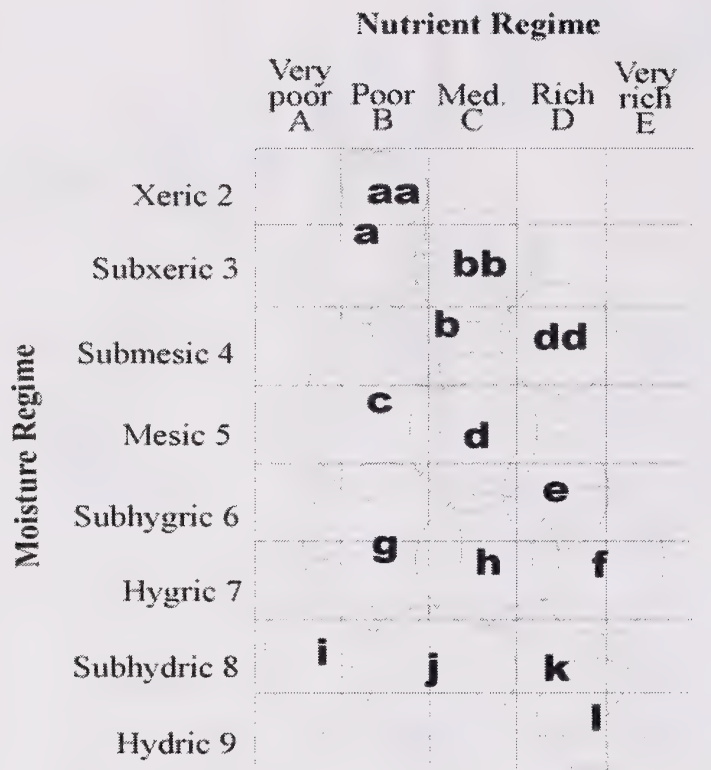

Ecological sites of the Dry Mixedwood subregion:

aa grassland

(xeric/poor)

a lichen

(subxeric/poor)

bb grassland

(subxeric/medium)

b blueberry

(submesic/medium)

c Labrador tea-mesic

(mesic/poor)

d low-bush cranberry

(mesic/medium)

dd grassland

(mesic/rich)

f horsetail

(hygric/rich)

g Labrador tea-subhygric

(subhygric/poor)

h Labrador tea/horsetail

(hygric/medium)

i bog

(subhygric/very poor)

j poor fen

(subhydric/medium)

$k$ rich fen

(subhydric/rich)

1 marsh

(hydric/rich)

e dogwood

(subhygric/rich) 


\section{GENERAL DESCRIPTION}

This ecosite is associated with small grassy openings within Jack pine and aspen forests. This site has dry conditions, with rapidly drained, nutrient poor soils. The parent materials are generally coarse textured eolian, glacialfluvial or fluvial eolian in origin. The high insolation and dry site conditions favour the growth of grassland species. These include Northern ricegrass, slender wheatgrass, Sedge, bearberry and plains wormwood In the moister sites (lower slope positions) aspen and shrubs ( saskatoon, rose) are quite common.

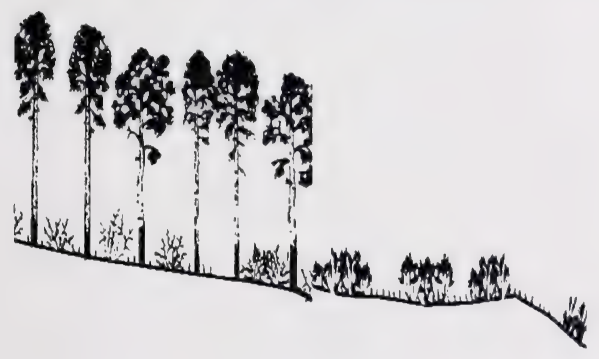

\section{SUCCESSIONAL RELATIONSHIPS}

Due to the nature of the site grasslands often remain the climax vegetation on these sites. In the moister lower slope positions shrubs often dominate the site with succession to aspen and spruce. On the drier hilltops and midslopes grasslands dominated by plains wormwood and northern ricegrass usually represent the climax vegetation. Heavy grazing pressure on the grasslands can often lead to a degraded site that is dominated by kentucky bluegrass on the moister sites.

\section{INDICATOR SPECIES}

\section{Saskatoon}

Rose

Snowberry

Beaked hazelnut

Plains wormwood

Bearberry

Strawberry

Sheep fescue

Northern ricegrass

Slender wheatgrass

Hairy wildrye

\section{SITE CHARACTERISTICS}

Moisture regime: xeric, subxeric, submesic

Nutrient regime: poor, medium

Topographic position: crest, upper, mid to lower slope Slope: $(0-2 \%)(5-10 \%)$

Aspect: south, southwest, west

\section{SOIL CHARACTERISTICS}

Organic thickness: $(0-2)$

Humus form: mor

Surface texture: SL, L

Effective texture: SL, S

Depth to Mottles/Gley: none

Drainage: rapid, well

Parent material: E, GF,FE,F

Soil subgroup: O.EB, E.DYB O.R, E.EB

\section{ECOSITE PHASES}

aal Plains wormwood (2) 


\section{RANGE PLANT COMMUNITY TYPES}

\section{CHARACTERISTIC SPECIES}

DMA3. Plains wormwood/Sedge

Forb

[ 8] Scouring rush

[ 12] Plains wormwood*

[1] Low goldenrod

[1] American vetch

[1] Yellow beardstongue

[ 10] Common yarrow

\section{Grasses}

[ 18 ] Sedge species*

[2] Sheep fescue*

[2] Creeping red fescue

[5] Kentucky bluegrass

\section{SITE CHARACTERISTICS}

Moisture regime: xeric, subxeric

Nutrient regime: poor,

Topographic position: crest, upper slope, midslope Slope: $5-10 \%, 10-20 \%$

Aspect: westerly, southerly

\section{SOIL CHARACTERISTICS}

\section{Organic thickness: $(0-2)$}

Humus form: mor

Surface texture: S, SL

Effective texture: $S$

Depth to Mottles/Gley: none

Drainage: rapid, well

Parent material: E, GF, FE

Soil subgroup: O.R, O.EB, E.EB 


\section{GENERAL DESCRIPTION}

This ecosite is associated with the south and west facing slopes along the Peace, Smoky and Wapiti rivers in the Dry Mixedwood subregion of Northwestern Alberta. This site has dry conditions, with rapidly drained, nutrient rich soils. The parent materials are generally glacio lacustrine, morainal, colluvial and fluvial in origin. The high insolation and dry site conditions favour the growth of grassland species. These include Western porcupine grass, Northern wheatgrass, Junegrass, Sedge and Fringed sage. In the moister draws aspen and shrubs (snowberry, saskatoon, chokecherry) are quite common.

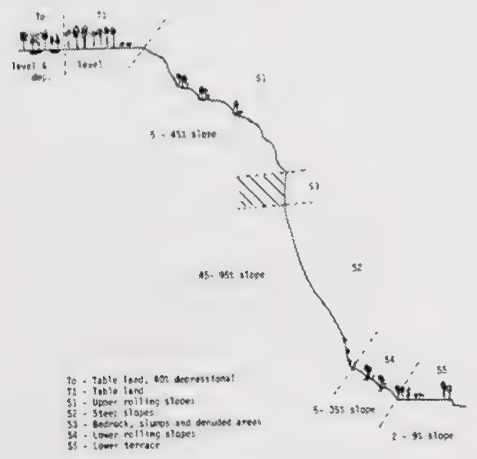

(Adams 1981)

\section{SUCCESSIONAL RELATIONSHIPS}

Due to the nature of the site grasslands often remain the climax vegetation on these sites. In the moister draws and lower slope positions aspen and spruce can succeed onto these grasslands. Frequent fire will often control the succession to trees in the moist areas. Heavy grazing pressure on the grasslands can often lead to a degraded site that is dominated by fringed sage, upland sedges and junegrass.

\section{INDICATOR SPECIES}

Western porcupine grass

Northern wheatgrass

Junegrass

Upland sedge

Green needlegrass

Saskatoon

Snowberry

\section{subxeric/medium}

\section{SITE CHARACTERISTICS}

Moisture regime: subxeric, submesic

Nutrient regime: poor, medium, rich

Topographic position: crest, upper slope, midslope

Slope: $27 \%, 45 \%, 90 \%$

Aspect: south, southwest, west

\section{SOIL CHARACTERISTICS}

Organic thickness: $(0-2)$

Humus form: mull

Surface texture: $\mathrm{L}, \mathrm{CL}$

Effective texture: C, SCL

Depth to Mottles/Gley: none

Drainage: rapid, well

Parent material: GF, M, C, F

Soil subgroup: O.BL, R.BL, O.MB,

S.GL,CA.DB,O.B,O.EB

\section{ECOSITE PHASES}

bb1 Western porcupine grass (7)

bb2 Northern wheatgrass (13) 


\section{bb1 Western porcupine grass $(n=7)$}

\section{CHARACTERISTIC SPECIES}

\section{Shrub}

[ 8 ] Fringed sage*

[1] Saskatoon

[2] Snowberry

Forb

[1] Little leaved everlasting

[1] White camas

[1] Loose flowered milkvetch

[2] Prairie crocus

[1] Wild blue flax

\section{Grasses}

\section{[ 15 ] Western porcupine grass*}

[15] Sedge species*

[ 5] Green needle grass*

[6] Junegrass*

[1] Western wheatgrass

[1] Kentucky bluegrass

[2] Northern wheatgrass

\section{SITE CHARACTERISTICS}

Moisture regime: subxeric, submesic

Nutrient regime: medium, rich

Topographic position: crest, upper slope, midslope

Slope: $25-35 \%, 35-72 \%$

Aspect: westerly, southerly

\section{SOIL CHARACTERISTICS}

Organic thickness: $(0-2)$

Humus form: mull

Surface texture: $\mathrm{L}, \mathrm{CL}$

Effective texture: $\mathrm{C}, \mathrm{SCL}$

Depth to Mottles/Gley: none

Drainage: rapid, well

Parent material: GF, M, C, F

Soil subgroup: O.BL, R.BL, O.MB, S.GL, CA.DB,O.B,

O.EB

\section{RANGE PLANT COMMUNITY TYPES}

DMA5. Western porcupine grass-Sedge/Fringed sage 
bb2 Northern wheatgrass $(n=13)$

\section{CHARACTERISTIC SPECIES}

Shrub

[6] Fringed sage*

[6] Saskatoon

[3] Snowberry

[3] Rose

Forb

[1] Little leaved everlasting

[1] Lindley's aster

[1] Showy locoweed

[1] Cut leaved anemone

[1] Wild blue flax

[2] Dandelion

\section{Grasses}

[3] Sedge species*

[2] Green needle grass*

[5] Junegrass*

[3] Western wheatgrass

[1] Richardson's needlegrass

[ 10] Northern wheatgrass*

\section{SITE CHARACTERISTICS}

Moisture regime: xeric, subxeric

Nutrient regime: poor, medium

Topographic position: crest, upper slope, midslope

Slope: $10-90 \%$

Aspect: westerly, southerly

\section{SOIL CHARACTERISTICS}

Organic thickness: $(0-2)$

Humus form: mull

Surface texture: L,CL

Effective texture: $\mathrm{C}, \mathrm{SCL}$

Depth to Mottles/Gley: none

Drainage: rapid, well

Parent material: GF, M, C, F

Soil subgroup: O.R, O.MB, O.EB, O.B, SZ.GL

\section{RANGE PLANT COMMUNITY TYPES}

DMA6. Northern wheatgrass-Junegrass/Fringed sage 


\section{CHARACTERISTIC SPECIES}

Tree

[ 4 ] Aspen

Shrub

[2] Beaked hazelnut

[17] Saskatoon*

[15] Snowberry*

[23 ] Rose*

Forb

[ 3 ] Northern bedstraw

[1] Strawberry

[2] Yellow peavine

[2] Lindley's aster

[2] American vetch

[1] Bearberry

[2] Common yarrow

\section{Grasses}

[4] Sedge species*

[1] Northern ricegrass

[3] Smooth brome

[2] Slender wheatgrass*

[1] Kentucky bluegrass

[2] Hairy wildrye

\section{SITE CHARACTERISTICS}

Moisture regime: submesic, mesic

Nutrient regime: medium

Topographic position: lower slope, midslope

Slope: 0-72\%

Aspect: westerly, southerly

\section{SOIL CHARACTERISTICS}

Organic thickness: (0-2)

Humus form: mor

Surface texture: L, SL

Effective texture: S, SL

Depth to Mottles/Gley: none

Drainage: rapid, well

Parent material: GF, M, C, F, GL

Soil subgroup: O.R, O.MB, O.EB, DG.SO, BR.GL 
Drainage: well, mod. well, imperfect

Parent material: GF, M, GL

Soil subgroup: O.GL, GR.GL, GL.GL

\section{CHARACTERISTIC SPECIES}

Tree

[48] Aspen*

[1] Balsam poplar

Shrub

[6] Raspberry

[1] Low bush cranberry

[4] Snowberry

[ 14] Rose*

Forb

[2] Northern bedstraw

[4] Strawberry*

[4] Yellow peavine

[4] Bunchberry*

[3] Lindley's aster

[3] Wild lily-of-the-valley*

[3] Dewberry

[4] Wintergreen*

[1] Dandelion*

[1] Clover species*

Grasses

[2] Marsh reedgrass

[3] Hairy wildrye

[1] Purple oatgrass*

[2] Slender wheatgrass*

[1] Kentucky bluegrass

\section{RANGE PLANT COMMUNITY TYPES}

DMC3. Aw/Rose/Low forb

DMC3a. Aw- $\mathrm{Pb} /$ Dandelion/Kentucky bluegrass

\section{SITE CHARACTERISTICS}

Moisture regime:, mesic

Nutrient regime: medium

Topographic position: mid, lower slope, level Slope: $0-5 \%$

Aspect: variable

\section{SOIL CHARACTERISTICS}

Organic thickness: (6-15), (0-5)

Humus form: mor, raw moder

Surface texture: SiL, SL, S, L

Effective texture: $\mathrm{C}, \mathrm{SiC}, \mathrm{CL}, \mathrm{SCL}, \mathrm{SiCL}$

Depth to Mottles/Gley: none, $(0-25)$ 
d1b Harvested Aw (n=4)

\section{CHARACTERISTIC SPECIES}

Tree

[ 20] Aspen

[ 1 ] Balsam poplar

Shrub

[ 5] Raspberry

[2] Saskatoon

[3] Snowberry

[ 19] Rose

[2] Low bush cranberry

Forb

[4] Northern bedstraw

[21 ] Strawberry

[1] Yellow peavine

[4] Lindley's aster

[1] American vetch

[4] Fireweed

[1] Bunchberry

Grasses

[ 17] Marsh reedgrass

[2] Northern ricegrass

[1] Hairy wildrye

[1] Slender wheatgrass

[2] Timothy

\section{SITE CHARACTERISTICS}

Moisture regime:, mesic

Nutrient regime: medium

Topographic position: mid, lower slope, level

Slope: $0-5 \%$

Aspect: variable

\section{SOIL CHARACTERISTICS}

Organic thickness: (6-15), (0-5)

Humus form: mor, raw moder

Surface texture: SiL, SL, S, L

Effective texture: C, SiC, CL, SCL, SiCL

Depth to Mottles/Gley: none, (0-25)

Drainage: well, mod. well, imperfect

Parent material: GF, M, GL
Soil subgroup: O.GL, GR.GL, GL.GL

\section{RANGE PLANT COMMUNITY TYPES}

DMC10. Deciduous cutblocks 


\section{GENERAL DESCRIPTION}

This ecosite is associated with the remnant prairies located throughout the Peace River district of Alberta. This site is associated with the dark colored solonetzic and chernomzic soils of the region. The parent materials are generally fine textured, slightly saline, fluvial, lacustrine or lacustrinetill in origin. The hard impermeable B horizon and slightly saline conditions tend to favour the growth of grassland species. These include Western porcupine grass, slender wheatgrass, sedge, California oatgrass and fringed sage Trees appear to be gradually moving into the old prairie remnants where the unfavorable characteristics of the solonetzic soils have been improved from many of the agricultural practices in the area.

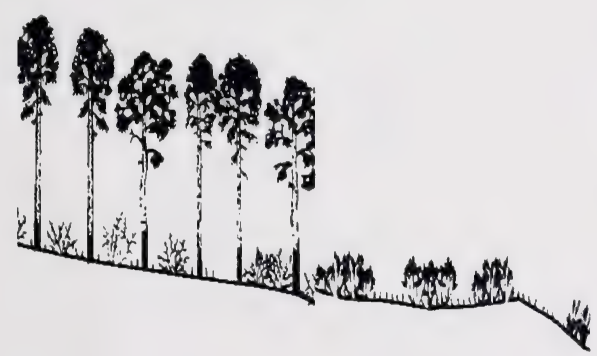

\section{SUCCESSIONAL RELATIONSHIPS}

Due to the nature of the site grasslands often remain the climax vegetation on these sites. However, industrial activities have greatly modified the original vegetation cover. Heavy grazing pressure on the remnant grasslands can often lead to a degraded site that is dominated by purple oatgrass, sedge, Kentucky bluegrass, dandelion and smooth brome.

\section{INDICATOR SPECIES}

\section{Saskatoon}

Rose

Snowberry

Strawberry

Veiny meadow rue

Dandelion

Common yarrow
California oatgrass

Sedge species

Kentucky bluegrass

Slender wheatgrass

Western porcupine grass

Purple oatgrass
Moisture regime: mesic, submesic

Nutrient regime: medium, rich

Topographic position: level, lower slope

Slope: $(0-5 \%)$

Aspect: south, southwest, west

\section{SOIL CHARACTERISTICS}

\author{
Organic thickness: $(0-5)$ \\ Humus form: mull \\ Surface texture: $\mathrm{L}, \mathrm{SiCL}$ \\ Effective texture: $\mathrm{C}, \mathrm{CL}$ \\ Depth to Mottles/Gley: none \\ Drainage: well, mod. well \\ Parent material: L \\ Soil subgroup: DB.SO, BL.SO, DB.SS, BL.SS, O.DB, \\ R.DB
}

\section{ECOSITE PHASES}

dd1 california oatgrass-slender wheatgrass

\section{mesic/rich}

\section{SITE CHARACTERISTICS}




\section{dd1 california oatgrass-slender wheatgrass $(n=6)$}

\section{CHARACTERISTIC SPECIES}

Shrub

[ 1] Saskatoon

[ 8] Snowberry*

[4] Rose

Forb

[2] Northern bedstraw

[9] Strawberry

[5] Common yarrow

[ 1 ] Three flowered avens

[ 5] Dandelion

[ 10] Veiny meadow rue*

[4] American vetch

\section{Grasses}

[6] California oatgrass*

[7] Western porcupine grass*

[9] Sedge species*

[14] Slender wheatgrass*

[ 8] Kentucky bluegrass

[3] Junegrass

[ 17] Purple oatgrass*

\section{SITE CHARACTERISTICS}

Moisture regime: mesic, submesic

Nutrient regime: medium, rich

Topographic position: level, lower slope

Slope: $(0-5 \%)$

Aspect: south, southwest, west

\section{SOIL CHARACTERISTICS}

Organic thickness: $(0-5)$

Humus form: mull

Surface texture: L, SiCL

Effective texture: $\mathrm{C}, \mathrm{CL}$

Depth to Mottles/Gley: none

Drainage: well, mod. well

Parent material: $\mathrm{L}$

Soil subgroup: DB.SO, BL.SO, DB.SS, BL.SS

\section{RANGE PLANT COMMUNITY TYPES}

DMA4. Purple oatgrass-California oatgrass-Sedge DMA4a. Veiny meadow rue/Slender wheatgrass-Fringed brome 
e4 dogwood shrubland $(n=10)$

\section{CHARACTERISTIC SPECIES}

Trees

[1] Balsam poplar

Shrub

[ 25] Red osier dogwood

[ 5] Snowberry*

[9] Rose

[32] Silverberry

Forb

[ 2] Horsetail

[3] Strawberry

[1] Common yarrow

[3] Veiny meadow rue*

[2] American vetch

Grasses

[3] Smooth brome

[3] Marsh reedgrass

[1] Sedge species*

[2] Kentucky bluegrass

\section{SITE CHARACTERISTICS}

Moisture regime: mesic, subhygric

Nutrient regime: rich

Topographic position: level, lower slope

Slope: (0-5\%)

Aspect: variable

\section{SOIL CHARACTERISTICS}

Organic thickness: (6-15)

Humus form: mor

Surface texture: $\mathrm{SiL}, \mathrm{Si}, \mathrm{SiC}, \mathrm{CL}$

Effective texture: $\mathrm{SiC}, \mathrm{C}$,

Depth to Mottles/Gley: (0-25)

Drainage: imperfect, poor, mod. well, well

Parent material: F, GL, M

Soil subgroup: O.LG, O.G, CU.R, GLCU.R

\section{RANGE PLANT COMMUNITY TYPES}

DMA17. Red osier dogwood/Marsh reedgrass

DMA 18. Silverberry/Smooth brome 


\section{CHARACTERISTIC SPECIES}

\section{RANGE PLANT COMMUNITY TYPES}

DMA12. Willow/Horsetail/Marsh reedgrass

DMA15. Sandbar willow

DMA16. Bebb willow/Marsh reedgrass

[65] Willow*

[1] Bracted honeysuckle

[10] Rose

Forb

[ 18] Horsetail*

[9] Arrow leaved coltsfoot

[ 8 ] Lindley's aster

[8] Bishop's cap

[5] Strawberry

[4] Veiny meadow rue

[4] Dewberry

[2] Fireweed

\section{Grasses}

$\begin{array}{ll}\text { [ 23 ] } & \text { Marsh reedgrass* } \\ \text { [1 ] } & \text { Hair-like sedge } \\ \text { [2] } & \text { Slender wheatgrass }\end{array}$

\section{SITE CHARACTERISTICS}

Moisture regime: subhygric, hygric, mesic

Nutrient regime: rich, medium

Topographic position: level, lower slope, toe

Slope: level (2-5\%)

Aspect: level, northerly

\section{SOIL CHARACTERISTICS}

Organic thickness: (6-15)

Humus form: mor

Surface texture: $\mathrm{SiL}, \mathrm{Si}, \mathrm{SiC}, \mathrm{CL}$

Effective texture: $\mathrm{SiC}, \mathrm{C}$,

Depth to Mottles/Gley: (0-25)

Drainage: imperfect, poor, mod. well, well

Parent material: F, GL, M

Soil subgroup: O.LG, O.G, CU.R, GLCU.R 


\section{CHARACTERISTIC SPECIES}

DMA13. River alder/Horsetail

Tree

[25] Paper birch*

[ 5] Larch

[3] White spruce

Shrub

[ 8 ] Bracted honeysuckle

[45] River alder*

[3] Willow

Forb

[ 27 ] Horsetail*

[6] Dewberry

[ 5 ] Bishop's cap

[3] Twinflower

[2] Sweet scented bedstraw

[1] Purple-stemmed aster

[1] American vetch

\section{Grasses}

[9] Marsh reedgrass*

[2] Sedge species

\section{SITE CHARACTERISTICS}

Moisture regime: subhygric, hygric, mesic

Nutrient regime: rich, medium

Topographic position: level, lower slope, toe

Slope: level (2-5\%)

Aspect: level, northerly

\section{SOIL CHARACTERISTICS}

\section{Organic thickness: (6-15)}

Humus form: mor

Surface texture: $\mathrm{SiL}, \mathrm{Si}, \mathrm{SiC}, \mathrm{CL}$

Effective texture: $\mathrm{SiC}, \mathrm{C}$,

Depth to Mottles/Gley: (0-25)

Drainage: imperfect, poor, mod. well, well

Parent material: F, GL, M

Soil subgroup: O.LG, O.G, CU.R, GLCU.R 


\section{CHARACTERISTIC SPECIES}

Shrub

[1] Sandbar willow

Forb

[ 1] Sea side arrowgrass

[1] Sea side buttercup

[ 1] Horsetail

\section{Grasses}

[12] Rush species

[20] Three square rush

[30 ] Prairie bulrush

[ 30 ] Nuttall's saltgrass

[25] Foxtail barley

\section{SITE CHARACTERISTICS}

Moisture regime: subhydric, hygric, hydric

Nutrient regime: medium, poor

Topographic position: level, lower slope, toe

Slope: level (2-5\%)

Aspect: level, northerly

\section{SOIL CHARACTERISTICS}

Organic thickness: $>80$

Humus form:

Surface texture: fibric, mesic

Effective texture: fibric, mesic, humic

Depth to Mottles/Gley: (0-25)

Drainage: imperfect, poor, very poor

Parent material: $\mathrm{O}, \mathrm{M}$

Soil subgroup: TY.M, R.G, TY.F, THU.M, R.HG, ME.OC
DMA25. Rush meadow

DMA27. Three square rush

DMA28. Prairie bulrush

DMA29. Nuttall's saltgrass

DMA30. Foxtail barley 


\section{CHARACTERISTIC SPECIES}

DMA24. Two stamened sedge

Shrub

[6] Bog willow

[1] Bog birch

Forb

[7] Buckbean

[5] Marsh cinquefoil

[3] Marsh marigold

Grasses

[ 82] Two stamened sedge

[1] Water sedge

\section{SITE CHARACTERISTICS}

Moisture regime: subhydric, hygric, hydric

Nutrient regime: medium, poor

Topographic position: level, lower slope, toe

Slope: level (2-5\%)

Aspect: level, northerly

\section{SOIL CHARACTERISTICS}

Organic thickness: $>80$

Humus form:

Surface texture: fibric, mesic

Effective texture: fibric, mesic, humic

Depth to Mottles/Gley: (0-25)

Drainage: imperfect, poor, very poor

Parent material: $\mathrm{O}, \mathrm{M}$

Soil subgroup: TY.M, R.G, TY.F, THU.M, R.HG, ME.OC 


\section{k2a grazed Willow $(n=13)$}

\section{CHARACTERISTIC SPECIES}

\section{Tree}

[ 1 ] Balsam poplar

\section{Shrub}

[ 1 ] Rose

[14] Willow*

Forb

[22] Dandelion*

[ 1 ] Clover*

[2] Mint

[1] Plantain

\section{Grasses}

[ 16] Kentucky bluegrass*

[ 12] Marsh reedgrass

[ 1 ] Foxtail barley

[1] Sedge species
Drainage: very poor, poor

Parent material: O, GL, L

Soil subgroup: R.G, R.HG, TY.F, O.F

\section{RANGE PLANT COMMUNITY TYPES}

DMA11. Willow/Marsh reedgrass-Kentucky bluegrass DMA14. Willow/Kentucky bluegrass/Dandelion

\section{SITE CHARACTERISTICS}

Moisture regime: hydric, subhydric, hygric

Nutrient regime: rich, medium, very rich

Topographic position: level, depression

Slope: level, (2-5\%)

Aspect: level

\section{SOIL CHARACTERISTICS}

Organic thickness: $>80,(6-15)$

Humus form: peatymor

Surface texture: fibric, C, mesic, SiL, humic

Effective texture: mesic, C,hC,fibric,SiC, humic

Depth to Mottles/Gley: (0-25) 


\section{RANGE PLANT COMMUNITY TYPES}

\section{CHARACTERISTIC SPECIES}

DMA9. Kentucky bluegrass-Rough hairgrass

Forb

[60] Dandelion*

[14] Strawberry*

[12] Yellow peavine

[11] Common yarrow

[ 7] Horsetail

[3] Smooth aster

[3] American vetch

Grasses

[ 18] Kentucky bluegrass*

[16] Rough hairgrass

[5] Slender wheatgrass

[4] Fringed brome

[2] Sedge species

\section{SITE CHARACTERISTICS}

Moisture regime: hydric, subhydric, hygric

Nutrient regime: rich, medium, very rich

Topographic position: level, depression

Slope: level, (2-5\%)

Aspect: level

\section{SOIL CHARACTERISTICS}

Organic thickness: $>80,(6-15)$

Humus form: peatymor

Surface texture: fibric, $\mathrm{C}$, mesic, $\mathrm{SiL}$, humic

Effective texture: mesic, $\mathrm{C}, \mathrm{hC}$, fibric, $\mathrm{SiC}$, humic

Depth to Mottles/Gley: (0-25)

Drainage: very poor, poor

Parent material: $\mathrm{O}, \mathrm{GL}, \mathrm{L}$

Soil subgroup: R.G, R.HG, TY.F, O.F 


\section{DRY MIXEDWOOD SUBREGION GRASSLAND AND SHRUBLAND COMMUNITY TYPES}

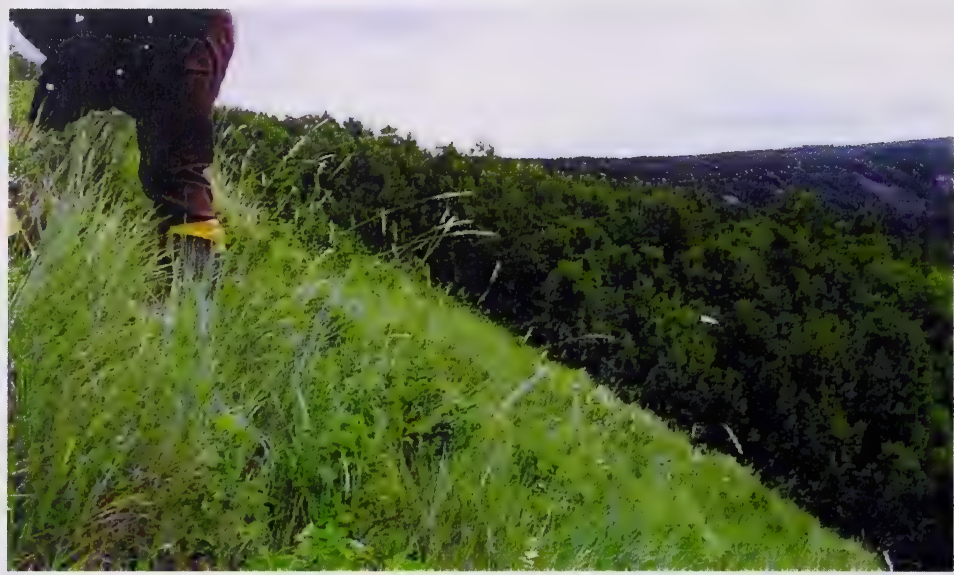

Photo 1. The Western porcupine grass-Sedge/Fringed sage community is found throughout the Dry Mixedwood subregion on the south-facing slopes of the Smoky, Wapiti and Peace Rivers. This community provides early spring forage for both wildlife and cattle.

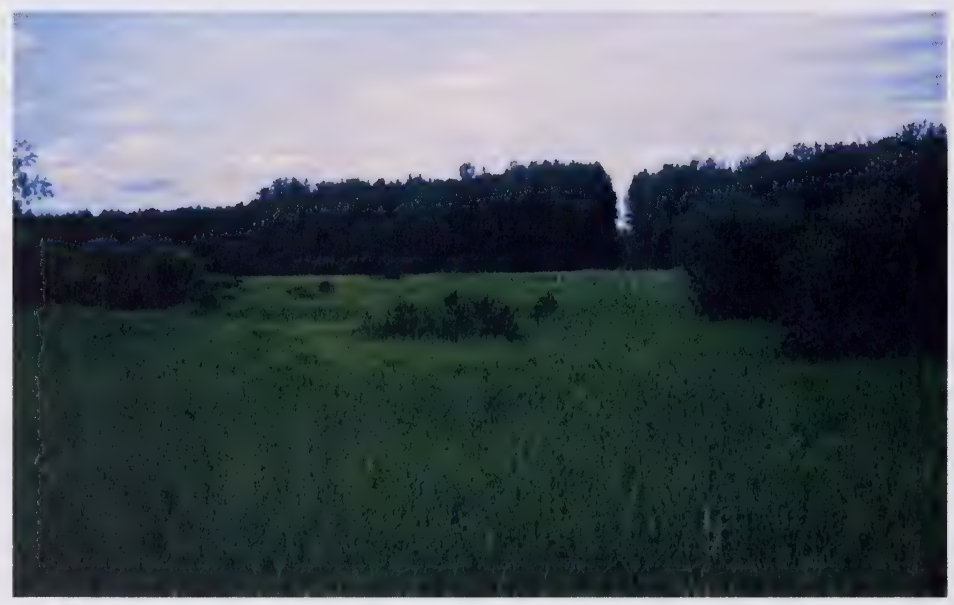

Photo 2. This picture represents the transition from sedge-marsh reedgrass meadows to willow sedge dominated community types. These community types provide a large amount of forage, but the moist conditions limit their use by livestock. 


\section{DM - NATIVE GRASS AND SHRUBLAND COMMUNITIES}

The Dry Mixedwood subregion represents the transition between the Boreal forest and Parkland subregions. Aspen Parkland-like vegetation can develop where site conditions or drought conditions occur in combination with the driest climatic conditions (Strong 1992). The Grande Prairie area is an example where a number of these conditions occur. It is within this area that a number of native upland grassland community types have been described. On steep, south-facing slopes of the Smoky, Wapiti and Peace Rivers with subxeric moisture regimes and medium nutrient regimes the Western porcupine grass-Sedge/Fringed sage and Northern wheatgrass/Fringed sage community types are common (Figure 1). The Purple oatgrass-SedgeCalifornia oatgrass community type is found on more upland sites with mesic moisture and medium nutrient regimes. Wilkinson and Johnston (1983) felt these grasslands to be the climax community type on Solonetzic soils. Indeed, Adams (1981) found the Western porcupine grassSedge dominated community on the Peace River slopes to be associated with Dark Gray Solods and Solonetzic Gray Luvisols. These grasslands provide important forage locally for both wildlife and domestic livestock. The grasslands of the south-facing river slopes are important spring forage sources because of early spring green-up.

On coarse textured, sandy soil, with submesic moisture and poor nutrient regimes which lack tree cover are found the Plains wormwood/Sedge and Saskatoon/Bearberry/Northern ricegrass community types. These community types are usually found in association with Jack pine dominated community types.

Wet freshwater (subhydric/rich) sites are associated with sedge, bulrush, cattail, creeping spike rush, swamp horsetail, common reedgrass, tall manna grass and marsh reedgrass dominated meadows. Sedge, bulrush, cattail, creeping spike rush, common reedgrass, tall manna grass and swamp horsetail species are usually associated with the areas of free standing water and reedgrass species tend to dominate the drier edges. Flat leaved willow and basket willow will invade into these meadows to form the Willow/Sedge and Willow/Marsh reedgrass community types. Rich, subhygric upland sites with better drainage are often dominated by Scouler's willow, Bebb's willow or red osier dogwood. These sites will often become dominated by trees in the absence of disturbance.

Boggy and acidic sites are often dominated by two stamened sedge and bog willow and will undergo succession to black spruce and larch in the absence of disturbance. A number of saline and alkaline sites were described in the Dry Mixedwood subregion. These sites are dominated by rush species, prairie bulrush, Nuttall's saltgrass, foxtail barley or three square rush. These saline communities are more common in the eastern part of the subregion. 


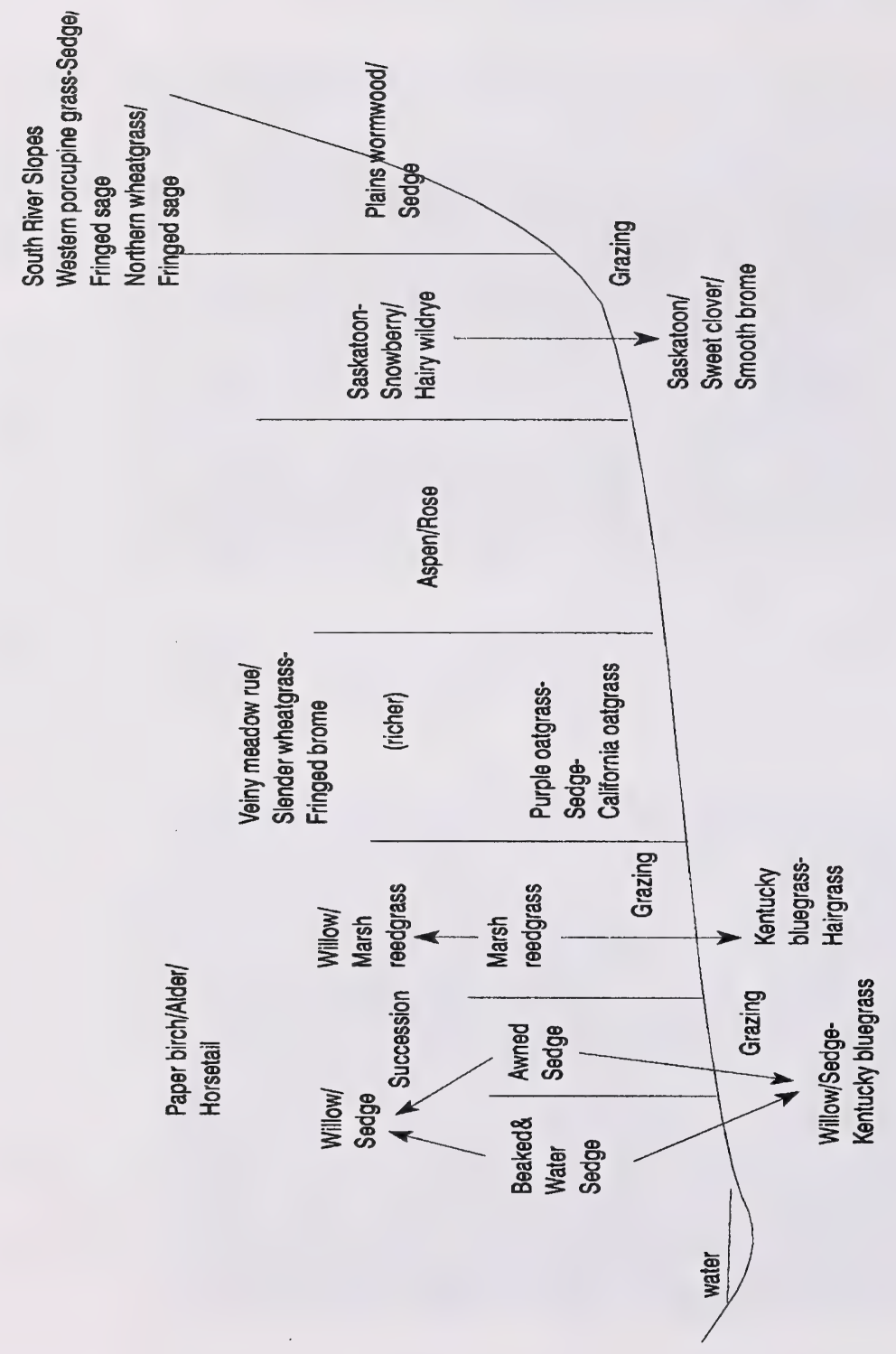

Figure 4. Overview of native grass and shrubland complex in the Dry Mixedwood subregion. 


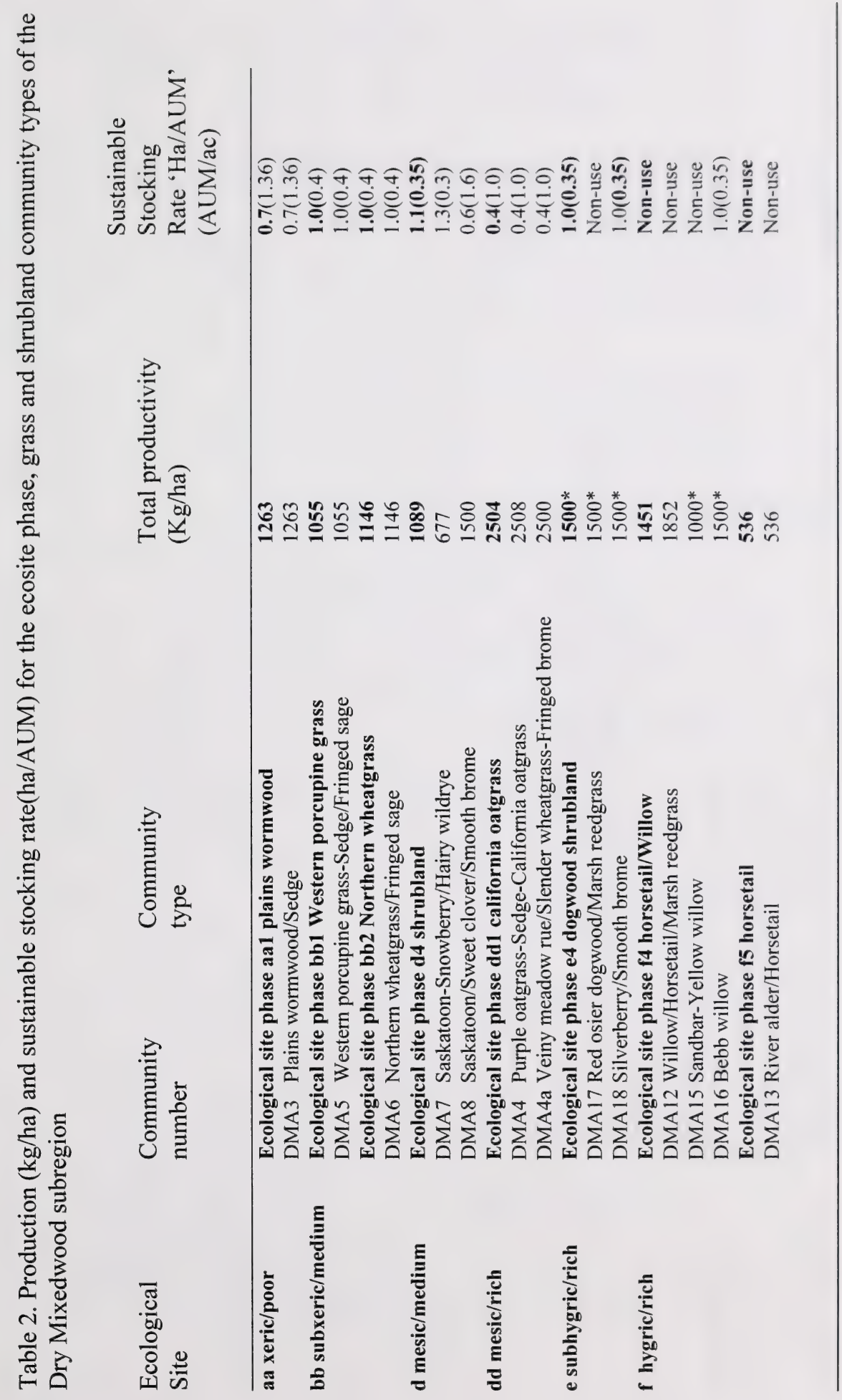




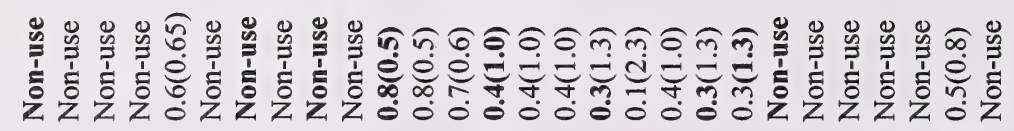

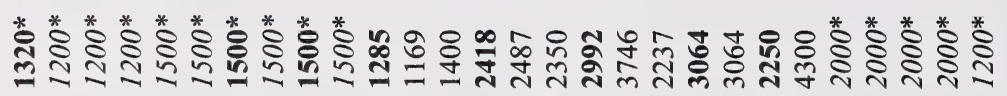

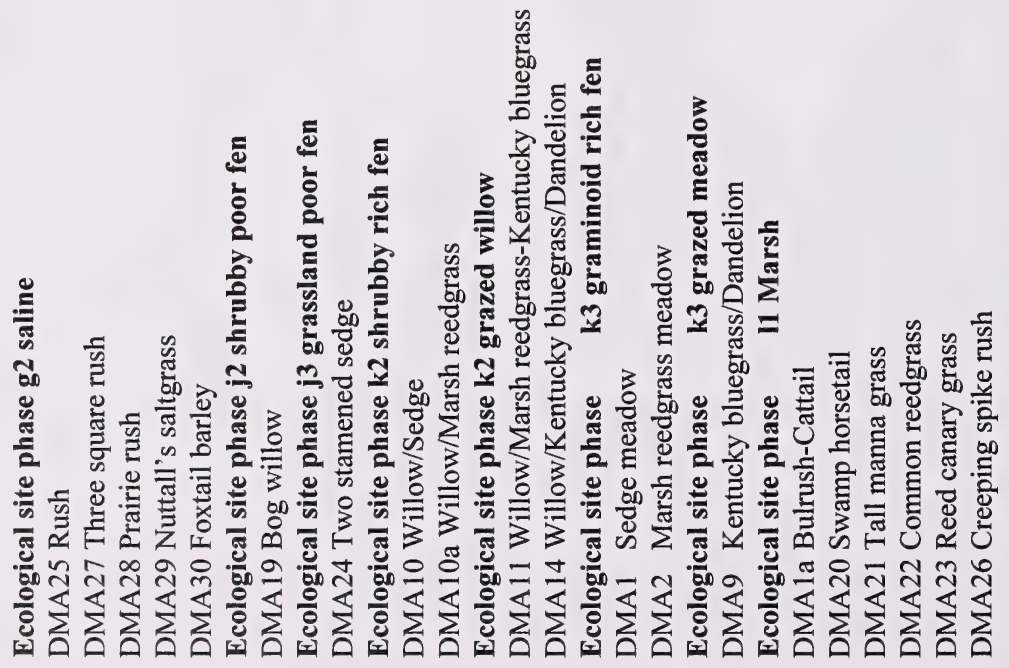

政

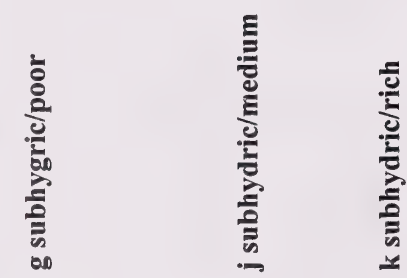

를 


\section{Dry Mixedwood Grass and Shrublands}

1. Shrub-dominated, willow, bog birch lowland sites or silverberry, river alder and red osier dogwood dominated upland sites......

Grass-dominated, or if shrub-dominated, upland species like hazelnut, saskatoon, or rose.

\section{SHRUB DOMINATED}

2. Red osier dogwood or river alder dominated sites..

Willow or silverberry dominated community types, sedge, marsh reedgrass, horsetail

dominate understory.

2a. Red osier dogwood dominated community.

River alder dominated community.

DMA17 Red osier dogwood/Marsh reedgrass

DMA13 River alder/Horsetail

3. Ungrazed communities dominated by horsetail, marsh reedgrass or sedge species, or moderately grazed community which has some Kentucky bluegrass. .4

Grazed community types dominated by Kentucky bluegrass and dandelion in the understory (little cover of native

species)...Willow/Kentucky Bluegrass/Dandelion (DMA14)

4. Ungrazed site, horsetail dominates understory. Willow/Horsetail/Marsh Reedgrass (DMA12)

Ungrazed or moderately grazed site, sedge or marsh reedgrass dominates understory.........5

.5. Wetland sedge species dominate understory.

Willow/Sedge (DMA10)

Upland sites dominated by willow or silverberry or boggy sites and riparian areas dominated by yellow willow, sandbar

willow or bog willow. $.5 \mathrm{a}$

5a Ungrazed or moderately grazed sites dominated by Marsh reedgrass in understory....... . .6

Willow or silverberry dominated uplands or willow dominated riparian areas or boggy areas......6a

6. Ungrazed marsh reedgrass dominates understory........Willow/Marsh Reedgrass (DMA10a) Moderately to heavily grazed sites with Kentucky bluegrass in understory

.Willow/Marsh Reedgrass-Kentucky Bluegrass (DMA 11)

6a Riparian areas dominated by sandbar and yellow willow DMA15 Sandbar - Yellow willow Upland sites dominated by Bebb willow, silverberry or boggy sites dominated by bog willow......6b

$6 \mathrm{~b}$ Boggy sites dominated by Bog willow.

DMA19 Bog willow

Upland sites dominated by Bebb willow or silverberry.

$6 \mathrm{c}$ Bebb willow dominated.

Silverberry dominated.

DMA16 Bebb willow/Marsh reedgrass

DMA18 Silverberry/Smooth brome

\section{GRASS DOMINATED}

7. Lowland sites dominated by sedge, marsh reed grass, Kentucky bluegrass, bulrush, swamp horsetail, creeping spike rush common reedgrass, reed canary grass or cattail (includes saline sites).

Upland sites or south facing slopes dominated by northern ricegrass, saskatoon, California oatgrass, sage, veiny meadow rue, slender wheatgrass or fringed brome.. .11

8. Ungrazed very wet sites dominated by native grass species. $8 \mathrm{a}$ Grazed sites dominated by Kentucky bluegrass..........................entucky Bluegrass/Dandelion (DMA9)

8a Saline sites dominated by three square rush, foxtail barley, Nuttall's saltgrass or baltic rush spp.....17 Fresh water or boggy sites dominated by cattails, bulrushes, sedge and Marsh reedgrass................9

9. Very wet sites with standing water, cattails, bulrush, swamp horsetail, tall manna grass, common reedgrass, reed canary grass, or creeping spike rush present. 9 a

Wet sites, dominated by sedge and marsh, narrow or northern reedgrass .10

9a. Bulrush or cattail dominated sites. DMA1 Bulrush-Cattail Drier sites, edge communities near free standing water. $.9 \mathrm{~b}$

9b Common reedgrass or reed canary grass dominated..................................................................9c

Tall manna grass, Creeping spike rush or swamp horsetail dominated...........................................9d

9c Common reedgrass dominated Reed canary grass dominated DMA23 Common reedgrass d Swamp horsetail dominated. DMA24 Reed canary grass Tall manna grass or creeping spike rush dominated

9e Tall manna grass dominated. DMA20 Swamp horsetail Creeping spike rush dominated.

DMA22 Tall manna grass

0 . Wet sites dominated by wetland sedge species DMA27 Creeping spike rush Drier sites dominated by marsh reedgrass. Marsh Reedgrass Meadow (DMA2)

10a Boggy sites dominated by two stamened sedge DMA25 Two stamened sedge Fresh water sites dominated by water, beaked or awned sedge.....DMA1 Sedge meadows 
11. Open meadow and grasslands or upland shrublands situated amongst jackpine and aspen stands. .12

South facing river slopes.

12. Mesic sites with medium to rich nutrient soils, dominated by purple oatgrass, sedge, slender wheatgrass, veiny meadow rue, fringed brome and California oatgrass. .13

Upland sandy sites, interspersed with jack pine or aspen

13. Mesic sites with medium nutrient regimes dominated by purple oatgrass, sedge, and California oatgrass species. Purple Oatgrass-Sedge-California Oatgrass (DMA4)

Richer sites, veiny meadow rue, slender wheatgrass, and fringed brome dominate the canopy. Veiny Meadow Rue/Slender Wheatgrass-Fringed Brome (DMA4a)

14. Very dry south facing hilltops dominated by Plains wormwood and sedge species

Plains Wormwood/Sedge (DMA3)

Moister sites dominated by hazelnut, saskatoon, bearberry, sweet clover, smooth brome, or northern ricegrass.

15. Native sites dominated by saskatoon

and snowberry

..Saskatoon-Snowberry/Hairy Wildrye (DMA7)

Grazed and disturbed sites dominated by saskatoon, sweet clover and smooth brome. Saskatoon/Sweet Clover/Smooth Brome (DMA8)

16. Moderate slopes dominated by western porcupine grass and sage. .Western Porcupine Grass-Sedge/Fringed Sage (DMA5) Very steep slopes or grazing modified communities dominated by northern wheatgrass and sage. .Northern Wheatgrass/Fringed Sage (DMA6)

17. Bulrush dominated sites

Nuttall's saltgrass, foxtail barley, or rush dominated

18. Prairie bulrush dominated

DMA29 Prairie bulrush

Three square rush dominated. DMA28 Three square rush

19. Nuttall's saltgrass dominated meadow DMA30 Nuttall's saltgrass Foxtail barley or Baltic rush dominated

20. Disturbed site dominated by foxtail barley DMA26 Rush 


\section{DMA1. Sedge meadows \\ (Carex aquatilis, C. rostrata, C. atherodes)}

$\mathbf{n = 4 1}$ This wetland community type is found near fresh water and can be dominated by water sedge. beaked sedge or awned sedge. The sedge meadow is a poorly drained community. As one moves to the drier edges marsh reedgrass becomes predominant. Willows will invade into both the sedge and marsh reedgrass dominated meadows. The sedge meadow community is very productive, but the high water table, particulary in the spring when the sedge species are most palatable, restricts livestock movement. One study done in the Yukon found that crude protein on these meadows declined from a high of $10 \%$ in May to less than $5 \%$ in September (Bailey et al. 1992).

Beaked sedge found in abundance in this community is usually associated with nitrogen rich conditions and moving water (Brierly et al. 1985). Water sedge is often found in abundance in this community type and is associated with calcium rich stagnant water (MacKinnon et al. 1992).

\section{PlaAN COMPOSITION Canopy COVER(\%)}

\section{SHRUBS}

MEAN RANGE CONST.

WILLOW SPP.

(Salix spp.)

$2 \quad 0-30 \quad 44$

\section{FORBS}

MARSH WILLOWHERB

(Epilobium palustris)

Dock

(Rumex acetosa)

SKULl CAP

(Scutellaria galericulata) 1

MINT

(Mentha arvensis)

\section{GRASSES}

BEAKED SEDGE

(Carex rostrata)

AWNED SEDGE

(Carex atherodes)

WATER SEDGE

(Carex aquatilis)

MARSH REEDGRASS

(Calamagrostis canadensis)3 $\quad 0-11 \quad 17$

\section{ENVIRONMENTAL VARIABLES}

\author{
MOISTURE REGIME (MEAN): \\ SUBHYDRIC-HYGRIC \\ NUTRIENT REGIME (MEAN \\ $\mathrm{RICH}$ \\ ELEVATION: \\ $586(579-600) \mathrm{M}$
}

SOIL DRAINAGE (MEAN):

POORLY TO VERY POORLY

RANGELAND HEALTH RATING:

HEALTHY

FORAGE PRODUCTION (KG/HA)

$$
\begin{array}{ll}
\text { GRASS } & 3673(1054-5028) \\
\text { FORB } & 73(0-80) \\
\text { SHRUB } & 40(0-120) \\
\text { TOTAL } & 3746(1254-5028)
\end{array}
$$

ECOLOGICALLY SUSTAINABLE STOCKING RATE

$0.1 \mathrm{Ha} / \mathrm{AUM}$ (Autumn only)(2.3 AUM/AC) 


\section{DMA1a. Bulrush-Cattail \\ (Scirpus acutus-Typha latifolia)}

$\mathbf{n = 1 8}$ This wetland community type is associated with standing water. This community is an emergent community found in standing water of ponds and sloughs. As one moves away from the water to the drier edges the sedge meadow communities are found. On the drier edges the marsh reedgrass community is found and willow are associated in the transition from the slough margin and the forest.

This community type would be rated as non-use for domestic livestock because of the extremely wet conditions.

\section{PLANT COMPOSITION CANOPY COVER(\%)}

MEAN RANGE CONST.

\section{FORBS}

ARUM-LEAVED ARROW HEAD

(Sagittaria cuneata) 1

NARROW LEAVED BURREED

(Sparganium eurycarpium)9

BULB BEARING WATER HEMLOCK

(Cicuta bulbifera)

\section{GRASSES}

COMMON GREAT BULRUSH

(Scripus validus)

GREAT BULRUSH

(Scirpus acutus)

CATTAIL

(Typha latifolia)

CREEPING SPIKE RUSH

(Eleocharis palustris)

SPANGLETOP

(Scholochloa festucacae) 5

\section{$0-3$}

17

$0-80 \quad 11$

0-3 11

6

29

27

$0-97$

$0-60$

11

$0-90 \quad 44$

$0-97 \quad 50$

3

$0-4$

22

0-97 5

\section{ENVIRONMENTAL VARIABLES}

MOISTURE REGIME (MEAN):

SUBHYDRIC-HYGRIC

NUTRIENT REGIME (MEAN

$\mathrm{RICH}$

ELEVATION:

$606 \mathrm{M}$

SOIL DRAINAGE (MEAN):

VERY POORLY

RANGELAND HEALTH RATING:

HEALTHY

FORAGE PRODUCTION (KG/HA)

GRASS 4300

TOTAL 4300

ECOLOGICALLY SUSTAINABLE STOCKING RATE

NON-USE 


\section{DMA2. Marsh reedgrass meadow \\ (Calamagrostis canadensis, C. inexpansa, C. stricta)}

$\mathbf{n}=\mathbf{1 2}$ This community is found on the edges of sedge meadows and moist draws where the water table is lower and can be dominated by either species of reedgrass. The lower water table makes this community accessible for most of the grazing season. Willow will invade onto these sites to form the Willow/Marsh reedgrass community type. Increased grazing pressure on these sites will cause marsh reedgrass to decline and their will be an invasion of Kentucky bluegrass and dandelion. These sites are highly productive for domestic livestock and should be rated as primary range.

\section{Plant COMPOSITION Canopy Cover(\%)} MEAN RANGE CONST.

\section{SHRUBS}

WILLOW SPP.

(Salix spp.)

$1 \quad 0-10 \quad 50$

FORBS

MINT

(Mentha arvense)

STINGING NETTLE

(Urtica dioica)

2

$0-20$

40

$3 \quad 0-10 \quad 33$

\section{GRASSES}

MARSH REEDGRASS

(Calamagrostis canadensis) 42

BALTIC RUSH

(Juncus balticus)

1

NORTHERN REEDGRASS

(Calamagrostis inexpansa) 10

WATER SEDGE

(Carex aquatilis)

NARROW REEDGRASS

(Calamagrostis stricta)
$1 \quad 0-3$

$15 \quad 0-70$

\section{ENVIRONMENTAL VARIABLES}

MOISTURE REGIME (MEAN):

SUBHYGRIC-HYGRIC

NUTRIENT REGIME (MEAN):

$\mathrm{RICH}$

ElEVATION:

603(600-606)M

SOIL DRAINAGE (MEAN):

POORLY

RANGELAND HEALTH RATING:

HEALTHY

\section{FORAGE PRODUCTION(KG/HA)}

$$
\begin{array}{ll}
\text { GRASS } & 1427(1254-1600) \\
\text { FORB } & 812(450-1174) \\
\text { TOTAL } & 2237(2050-2424)
\end{array}
$$

ECOLOGICALLY SUSTAINABLE STOCKING RATE $0.4 \mathrm{HA} / \mathrm{AUM}$ (1.0 AUM/AC) 


\section{DMA3. Plains wormwood/Sedge \\ (Artemisia campestris/Carex spp.)}

$\mathbf{n}=\mathbf{2}$ This community type is found on coarse textured, sandy soils. It is generally found on hilltops and southfacing slopes in openings among Jack pine on the uplands and black spruce in the lowlands. This community type was also described on similar site conditions in the Central Mixedwood subregion. This community would be considered either secondary or non-use range for domestic livestock because of the low forage production and fragile nature of the community.

\section{PlaAT COMPOSITION CANOPY COVER(\%)} MEAN RANGE CONST.

\section{FORBS}

SCOURING RUSH

(Equisetum hyemale)

PLAINS WORMWOOD

(Artemisia campestris) $\quad 12 \quad 8-15 \quad 100$

LOW GOLDENROD

(Solidago missouriensis) $110-2 \quad 50$

AMERICAN VETCH

(Vicia americana)

YELLOW BEARDSTONGUE

(Penstemon confertus)

GRASSES

KENTUCKY BLUEGRASS

(Poa pratensis)

CREEPING RED FESCUE

(Festuca rubra)

SEDGE

(Carex spp)

SHEEP FESCUE

(Festuca saximontana)
8

0-16 50

$1 \quad 0-2 \quad 50$

$1 \quad 0-1 \quad 50$

$5 \quad 0-9 \quad 50$

$2 \quad 0-4 \quad 50$

$18 \quad 1-34 \quad 100$

$2 \quad 1-3 \quad 100$

\section{ENVIRONMENTAL VARIABLES}

MOISTURE REGIME (MEAN): XERIC-SUBXERIC

NUTRIENT REGIME (MEAN): SUBMESOTROPHIC

ELEVATION:

$$
\text { 467(325-606) M }
$$

SOIL DRAINAGE:

RAPIDLY TO WELL

SLOPE(RANGE):

$$
16(10-22)
$$

ASPECT:

SOUTH TO WESTERLY

RANGELAND HEALTH RATING:

HEALTHY

FORAGE PRODUCTION (KG/HA)

GRASS 652

FORB 525

SHRUB 86

TOTAL 1263

ECOLOGICALLY SUSTAINABLE STOCKING RATE

$0.7 \mathrm{HA} / \mathrm{AUM}(1.4 \mathrm{AUM} / \mathrm{AC})$ 


\section{DMA4. Purple oatgrass-Sedge-California oatgrass \\ (Schizachne purpurascens-Carex spp.-Danthonia californica)}

$\mathbf{n = 4} \quad$ This community appears to be characteristic of dry grassy meadows on dark colored Solonetzic soils and gentle to level areas throughout the Dry Mixedwood subregion. Wilkinson and Johnson (1982), found there was a close correlation between large tracts of prairie vegetation and the distribution of solonetzic soils in the Peace River district of Alberta. They specifically described Western porcupine grass-Sedge/Fringed sage community on steep south -facing slopes and a Sedge-California oatgrass-Western porcupine grass on more gentle slopes. They felt the solonetzic soils supported grasslands and not forests because of their unfavourable ratios of $\mathrm{Ca}$ and $\mathrm{Na}$, hard, columnar B-horizon, and relatively impermeable clay pan close to the surface. This community type appears to more similar to their Sedge-California oatgrass-Western porcupine grass community type. It is likely the heavy grazing pressure of the described sites favours the growth of purple oatgrass over Western porcupine grass on these sites.

This community type would be rated as primary range. Indeed many of the sites described were old homestead sites.

Plant Composition Canopy Cover(\%) MEAN RANGE CONST.

SHRUBS

PRICKLY ROSE

(Rosa acicularis)

SNOWBERRY

(Symphoricarpos

occidentalis)

SASKATOON

(Amelanchier alnifolia)

FORBS

STRAWBERRY

(Fragaria virginiana)

MEADOW RUE

(Thalictrum venulosum)

100DANDELION

(Taraxacum officinale) $\quad 8 \quad 0-20 \quad 100$

YARROW

(Achllea millefolium)

AMERICAN VETCH

(Vicia americana)

GRASSES

PURPLE OATGRASS

(Schizachne purpurascens)25

SLENDER WHEATGRASS

(Agropyron trachycaulum) $12 \quad 6-18 \quad 100$

KENTUCKY BLUEGRASS

(Poa pratensis)

PRAIRIE SEDGE

(Carex prairea)

JUNEGRASS

(Koeleria macrantha)

CALIFORNIA OATGRASS
(Danthonia californica) $9 \quad 0-28 \quad 50$

\section{ENVIRONMENTAL VARIABLES}

MOISTURE REGIME (MEAN): MESIC

NUTRIENT REGIME (MEAN): MEDIUM

ELEVATION:

576-606(584) M

SOIL DRAINAGE (MEAN):

WELL

SLOPE \% (RANGE): 2(0-5)

ASPECT:

SOUTH TO WEST

RANGELAND HEALTH RATING:

HEALTHY WITH PROBLEMS

FORAGE PRODUCTION (KG/HA)

GRASS $1463(626-2578)$

FORB $818(500-1192)$

SHRUB 227(0-606)

TOTAL 2508(1600-3316)

ECOLOGICALLY SUSTAINABLE STOCKING RATE $0.4 \mathrm{HA} / \mathrm{AUM}$ (1.0 AUM/AC) 


\section{DMA4a. Veiny meadow rue/Slender wheatgrass-Fringed brome (Thalictrum venulosum/Agropyron trachycaulum-Bromus ciliatus)}

$\mathbf{n}=\mathbf{2}$ This community appears to be characteristic of dry grassy meadows on dark colored Chernozemic soils and gentle to level areas throughout the Dry Mixedwood subregion. This community type is likely associated with the large tracts of prairie vegetation described by Wilkinson and Johnson (1982) in the Peace River district of Alberta. They specifically described Western porcupine grass-Sedge/Fringed sage community on steep south facing slopes and a Sedge-California oatgrass-Western porcupine grass on more gentle slopes. They felt these grasslands were associated with the distribution of solonetzic soils in the Peace River area. This community type appears to be richer than the Sedge-California oatgrass-Western porcupine grass community described by Wilkinson and Johnson. The soils on this community are described as Chernozemic and the parent material is fluvial in origin.

These sites are very productive and should be rated as primary range.

\section{PLANT COMPOSITION CANOPY COVER(\%) \\ MEAN RANGE CONST.}

TREES

WHITE SPRUCE

(Picea glauca)

$1 \quad 0-1 \quad 50$

FORBS

STRAWBERRY

(Fragaria virginiana) $\quad 1 \quad 0-1 \quad 50$

MEADOW RUE

(Thalictrum venulosum) $\quad 23 \quad 15-30 \quad 100$

FIREWEED

(Epilobium angustifolum) $2 \quad 1-2 \quad 100$

YARROW

(Achllea millefolium) $\quad 1 \quad 0-2 \quad 50$

TALL LUNGWORT

(Mertensia paniculata) $\quad 9 \quad 2-15 \quad 100$

GRASSES

FRINGED BROME

(Bromus cilatus)

$15 \quad 10-20 \quad 100$

SLENDER WHEATGRASS

(Agropyron trachycaulum) $18 \quad 15-20 \quad 100$

WHITE SCALED SEDGE

(Carex xerantica)

10

9-10

100

MARSH REEDGRASS

(Calamagrostis canadensis) 1

$0-2 \quad 50$

\section{ENVIRONMENTAL VARIABLES}

MOISTURE REGIME (MEAN):

MESIC

NUTRIENT REGIME (MEAN): MEDIUM-RICH

ELEVATION:

472-587(530) M

SOIL DRAINAGE (MEAN):

MODERATELY WELL

SLOPE:

LEVEL

RANGELAND HEALTH RATING:

HEALTHY

FORAGE PRODUCTION (KG/HA)

TOTAL 2500 KG/HA *ESTIMATE

ECOLOGICALLY SUSTAINABLE STOCKING RATE $0.4 \mathrm{HA} / \mathrm{AUM}(1.0 \mathrm{AUM} / \mathrm{AC})$ 


\section{DMA5. Western porcupine grass-Sedge/Fringed sage (Stipa curtiseta-Carex spp./Artemisia frigida)}

$\mathbf{n}=7$ This community type is found on steep, south-facing slopes along the banks of the Peace, Smoky and Wapiti rivers throughout the Dry Mixedwood subregion. Wilkinson and Johnson (1982), found there was a close correlation between large tracts of prairie vegetation and the distribution of solonetzic soils in the Peace River district of Alberta. They specifically described Western porcupine grass-Sedge/Fringed sage community on steep south -facing slopes and a Sedge-California oatgrass-Western porcupine grass on more gentle slopes. They felt the solonetzic soils supported grasslands and not forests because of their unfavourable ratios of $\mathrm{Ca}$ and $\mathrm{Na}$, hard, columnar B-horizon, and relatively impermeable clay pan close to the surface. Adams (1981), found this community type as being a major source of spring forage for livestock in the Peace River area. He found that with increased grazing pressure sedge, junegrass, northern and western wheatgrass would increase as western porcupine grass declines. Often this community type is on steep enough slopes to be considered non-use for domestic livestock.

\section{Plant COMPOSITION Canopy Cover $(\%)$} MEAN RANGE CONST.

\section{SHRUBS}

FRINGED SAGE

(Artemisia frigida)

SASKATOON

(Amelanchier alnifolia) $\quad 1 \quad 0-2 \quad 71$

SNOWBERRY

(Symphoricarpos

occidentalis)

2

FORBS

LITTLE LEAVED EVERLASTING

(Antennaria parviflora)

BASTARD'S TOADFLAX

(Commandra umbellata) 1

PRAIRIE CROCUS

(Anemone patens)

$2 \quad 0-12 \quad 43$

PRICKLY PEAR CACTUS

(Opuntia fragilis)

1

GRASSES

WESTERN PORCUPINE GRASS

(Stipa curtiseta) 15

BLUNT SEDGE

(Carex obtusata)

GREEN NEEDLEGRASS

(Stipa viridula) 5

JUNEGRASS

(Koeleria macrantha) 6

WESTERN WHEATGRASS

(Agropyron smithii)

KENTUCKY BLUEGRASS

(Poa pratensis)

NORTHERN WHEATGRASS

(Agropyron dasystachyum)2

\section{ENVIRONMENTAL VARIABLES}

\section{MOISTURE REGIME (MEAN):} SUBXERIC-SUBMESIC

NUTRIENT REGIME (MEAN): POOR-MEDIUM

ELEVATION:

$$
\text { 442-606(503) M }
$$

SOIL DRAINAGE (MEAN): VERY RAPIDLY

SLOPE:

$$
35-82(59) \%
$$

ASPECT:

\section{SOUTH AND WEST}

RANGELAND HEALTH RATING: HEALTHY

\section{FORAGE PRODUCTION(KG/HA)}

GRASS $989(700-945)$

FORB 254(0-531)

SHRUB 5(0-20)

TOTAL $1055(752-1476)$

ECOLOGICALLY SUSTAINABLE STOCKING RATE $1.0 \mathrm{HA} / \mathrm{AUM}(0.4 \mathrm{AUM} / \mathrm{AC})$ 


\section{DMA6. Northern wheatgrass-Junegrass/Fringed sage \\ (Agropyron dasystachyum-Koeleria macrantha/Artemisia frigida)}

$\mathbf{n = 1 3}$ This community type is found on steep, south-facing slopes along the banks of the Peace, Smoky and Wapiti rivers throughout the Dry Mixedwood subregion. Adams (1981), felt this community type would form when the Western porcupine grass community was heavily to moderately grazed, but a number of plots were described in an area that had little grazing pressure. This community was located on a much steeper slope $(76 \%$ vs $35 \%$ ) than the previously described Western porcupine grass community type. It is likely that the drier site conditions and shallower and poorer nutrient soils favour the growth of northern wheatgrass over Western porcupine grass. This community type is located on a steep enough slope to be considered non-use for domestic livestock.

\section{PlaANT COMPOSITION CANOPY COVER(\%) \\ MEAN RANGE CONST.}

\section{SHRUBS}

FRINGED SAGE

(Artemisia frigida)

SASKATOON

(Amelanchier alnifolia)

ROSE

(Rosa acicularis)

SNOWBERRY

(Symphoricarpos

occidentalis)

FORBS

WILD BLUE FLAX

(Linum lewesii)

LINDLEY'S ASTER

(Aster ciliolatus)

SHOWY LOCOWEED

(Oxytropis splendens)

DANDELION

(Taraxacum offincinale) 1

GRASSES

NORTHERN WHEAT GRASS

(Agropyron dasystachyum )10

SEDGE SPP.

(Carex spp.)

RICHARDSON NEEDLEGRASS

(Stipa richardsonii) 1

JUNEGRASS

(Koeleria macrantha) 5

SLENDER WHEATGRASS

(Agropyron trachycaulum) 3
$6 \quad 0-20 \quad 80$

$6 \quad 0-15 \quad 95$

$3 \quad 0-15 \quad 62$

$3 \quad 0-10 \quad 69$

$1 \quad 0-4 \quad 23$

$1 \quad 0-3 \quad 46$

$0-2 \quad 39$

$0-2 \quad 62$

$0-17 \quad 75$

$0-7 \quad 63$

$0-4 \quad 15$

$0-20 \quad 77$

$0-30 \quad 46$

\section{ENVIRONMENTAL VARIABLES}

MOISTURE REGIME (MEAN):

XERIC-SUBXERIC

NUTRIENT REGIME (MEAN): POOR

ELEVATION: 345-606 M

SOIL DRAINAGE (MEAN): VERY RAPIDLY

SLOPE:

$68(10-90 \%)$

ASPECT:

SOUTH AND WEST

RANGELAND HEALTH RATING: HEALTHY

FORAGE PRODUCTION (KG/HA)

GRASS $600(500-798$

FORB 183(50-400)

SHRUB 309(220-450)

TOTAL 1146(1000-1350)

ECOLOGICALLY SUSTAINABLE STOCKING RATE $1.0 \mathrm{HA} / \mathrm{AUM}(0.4 \mathrm{AUM} / \mathrm{AC})$ 


\section{DMA7. Saskatoon-Snowberry/Hairy wildrye (Amelanchier alnifolia-Symphoricarpos occidentalis/Elymus innoratus)}

$\mathbf{n}=9 \quad$ This community represents small shrubby openings within aspen forests on southwest facing slopes and level areas. These sites have well developed Luvisolic soils with colluvial, glacialfluvial and glacial lacustrine parent materials. It is likely these shrubby openings are drier than the surrounding forest, which favours the growth of shrubs over trees. Forage productivity on these sites is only moderate averaging only $677 \mathrm{~kg} / \mathrm{ha}$. These sites are also heavily utilized by wildlife. As a result caution should be used when managing these sites for domestic livestock grazing in order to prevent overutilization.

\section{PLANT COMPOSITION CANOPY COVER(\%)} MEAN RANGE CONST.

\section{SHRUBS}

BLUEBERRY

(Vaccinium myrtilloides) $1 \quad 0-5 \quad 22$

CHOKECHERRY

$\begin{array}{llll}\text { (Prunus virginiana) } & 12 & 0-45 \quad 78\end{array}$

SNOWBERRY

(Symphoricarpos

occidentalis)

SASKATOON

(Amelanchier alnifolia)

PRICKLY ROSE

(Rosa acicularis)

FORBS

BEARBERRY

(Arctostaphylos uva-ursi)

STRAWBERRY

(Fragaria virginiana)

YELLOW PEAVINE

(Lathyrus ochroleucus) $\quad 1 \quad 0-2 \quad 78$

LINDLEY'S ASTER

(Aster ciliolatus)

GRASSES

NORTHERN RICEGRASS

(Oryzopsis pungens)

SLENDER WHEATGRASS

$10 \quad 1-30 \quad 100$

$15 \quad 6-65 \quad 100$

$13 \quad 5-27 \quad 100$

(Agropyron trachycaulum)3

BLUNT SEDGE

(Carex obtusata)

HAIRY WILDRYE

(Elymus innovatus)

KENTUCKY BLUEGRASS

(Poa pratensis)
$0-36 \quad 22$

$0-7 \quad 67$

$2 \quad 0-5 \quad 67$

$0-5 \quad 56$

$0-9 \quad 22$

$2 \quad 0-10 \quad 78$

$1 \quad 0-7 \quad 11$
$2 \quad 0-12 \quad 22$

\section{ENVIRONMENTAL VARIABLES}

MOISTURE REGIME (MEAN):

MESIC-SUBMESIC

NUTRIENT REGIME (MEAN): POOR

ELEVATION: $343-606(460) M$

SOIL DRAINAGE (MEAN): VERY RAPIDLY TO WELL

SLOPE (RANGE): 17(0-72)

ASPECT:

VARIABLE

RANGELAND HEALTH RATING:

HEALTHY

FORAGE PRODUCTION(KG/HA)

GRASS 344(124-564)

FORB 189(82-296)

SHRUB 144(104-184)

TOTAL $677(524-830)$

ECOLOGICALLY SUSTAINABLE STOCKING RATE $1.3 \mathrm{Ha} / \mathrm{AUM}(0.3 \mathrm{AUM} / \mathrm{AC})$ 


\section{DMA8. Saskatoon/Sweet clover/Smooth brome (Amelanchier alnifolia/Meliolatus officinalis/Bromus inermis)}

$\mathbf{n}=\mathbf{2}$ This community type appears to represent the Saskatoon-Snowberry/Hairy wildrye community type which has undergone disturbance by livestock. Sweet clover and smooth brome are both weedy species and occur essentially where roads and settlement occurs. Sweet clover is well adapted to growing on roadsides and in waste places. Unfortunately, forage production was not done for this community type, but sweet clover and brome can be very productive if grazed before they become overmature.

\section{PLANT COMPOSITION CANOPY COVER(\%)} MEAN RANGE CONST.

TREES

ASPEN

$\begin{array}{llll}\text { (Populus tremuloides) } & 5 & 4-5 & 100 \\ \text { SHRUBS } & \end{array}$

SNOWBERRY

(Symphoricarpos

occidentalis)

PRICKLY ROSE

(Rosa acicularis)

SASKATOON

$\begin{array}{llll}\text { (Amelanchier alnifolia) } & 7 & 1-13 & 100\end{array}$

FORBS

STRAWBERRY

$\begin{array}{llll}\text { (Fragaria virginiana) } & 1 & 1-2 & 100\end{array}$

CREAM COLORED VETCHLING(PEAVINE)

$\begin{array}{llll}\text { (Lathyrus ochroleucus) } & 2 & 1-3 & 100\end{array}$

NORTHERN BEDSTRAW

$\begin{array}{llll}\text { (Galium boreale) } & 5 & 1-8 & 100\end{array}$

SWEET CLOVER

$\begin{array}{llll}\text { (Meliolatus officinalis) } & 8 & 0-16 & 50\end{array}$

GRASSES

SMOOTH BROME

(Bromus inermis)

ROSS'S SEDGE

(Carex rossii)

TIMOTHY

(Phleum pratense)

KENTUCKY BLUEGRASS

(Poa pratensis)

$20 \quad 14-25 \quad 100$

$37 \quad 11-62 \quad 100$

00

50

$7 \quad 0-13 \quad 50$

$6 \quad 4-7 \quad 100$

$5 \quad 0-6 \quad 50$

$1 \quad 0-1 \quad 50$

\section{ENVIRONMENTAL VARIABLES}

MOISTURE REGIME (MEAN):

SUBMESIC-MESIC

NUTRIENT REGIME (MEAN):

POOR-MEDIUM

ELEVATION:

$455 \mathrm{M}$

SOIL DRAINAGE (MEAN):

WELL TO MODERATELY WELL

SLOPE (RANGE):

4(3-5)

ASPECT:

SOUTHERLY

RANGELAND HEALTH RATING: UNHEALTHY

FORAGE PRODUCTION(KG/HA)

TOTAL $1500 *$ ESTIMATE

ECOLOGICALLY SUSTAINABLE STOCKING RATE

$0.6 \mathrm{HA} / \mathrm{AUM}(0.65 \mathrm{AUM} / \mathrm{AC})$ 


\section{DMA9. Kentucky bluegrass/Dandelion \\ (Poa pratensis/Taraxacum officinale)}

$\mathbf{n}=\mathbf{2} \quad$ This community type represents a Marsh reedgrass meadow that has undergone heavy prolonged grazing pressure and is now dominated by Kentucky bluegrass, rough hairgrass and dandelion. This community is a fairly productive community type and the species are generally palatable to livestock when grazed in the vegetative state. but the extremely heavy grazing pressure which is needed to displace the native grass species indicates that there are livestock distribution problems that should be addressed.

\section{PLANT COMPOSITION CANOPY COVER(\%)} MEAN RANGE CONST.

\section{FORBS}

AMERICAN VETCH

(Vicia americana)

DANDELION

(Taraxacum officinale)

YELLOW PEAVINE

(Lathyrus ochroleucus)

WILD STRAWBERRY

(Fragaria virginiana)

YARROW

(Achillea millefolium)

HORSETAIL

(Equisetum arvense)

GRASSES

KENTUCKY BLUEGRASS

(Poa pratensis)

ROUGH HAIRGRASS

(Agrostis scabra)

SLENDER WHEATGRASS

(Agropyron trachycaulum) 3

FRINGED BROME

(Bromus ciliatus)

$\begin{array}{ccc}3 & 3-4 & 100 \\ 30 & 0-60 & 50 \\ 6 & 0-12 & 50 \\ 7 & 0-14 & 50 \\ 6 & 0-11 & 50 \\ 4 & 0-7 & 50 \\ 58 & 18-97 & 100 \\ 8 & 0-15 & 50 \\ 2 & 0-5 & 50 \\ 2 & 0-4 & 50\end{array}$

\section{ENVIRONMENTAL VARIABLES}

MOISTURE REGIME (MEAN): HYGRIC-SUBHYGRIC

NUTRIENT REGIME (MEAN): $\mathrm{RICH}$

ELEVATION: $697 \mathrm{M}$

SOIL DRAINAGE (MEAN): IMPERFECTLY

RANGELAND HEALTH RATING: UNHEALTHY

FORAGE PRODUCTION(KG/HA)

$\begin{array}{ll}\text { GRASS } & 1382 \\ \text { FORB } & 1682 \\ \text { TOTAL } & 3064\end{array}$

ECOLOGICALLY SUSTAINABLE STOCKING RATE $0.3 \mathrm{HA} / \mathrm{AUM}$ (1.3 AUM/AC) 


\section{DMA10. Willow/Sedge \\ (Salix spp./Carex spp.)}

$\mathbf{n}=\mathbf{2 7}$ This community type is found along the edges of sedge meadows and in moist depressions. Generally flat leaved willow and basket willow become established at the edges of the sedge meadows due to the shorter duration of standing water. Increased flooding and prolonged water logging may result in the disappearance of willow and a transition to a water sedge meadow.

These sites are fairly productive but difficult to graze due to the moist ground conditions and heavy shrub cover which reduces access and mobility within the area.

\section{Plant COMPOSITION CANopy COVER(\%)} MEAN RANGE CONST.

\section{SHRUBS}

BEBB WILLOW

(Salix bebbiana)

$5 \quad 0-65 \quad 47$

Flat LEAVED WILLOW

(Salix planifolia)

BASKET WILLOW

(Salix petiolaris)

$11 \quad 0-90 \quad 52$

$7 \quad 0-60$

37

FORBS

MiNT

(Mentha arvensis)

$1 \quad 0-5 \quad 44$

SKULLCAP

$\begin{array}{llll}\text { (Scutellaria galericulata) } & 1 & 0-10 & 52\end{array}$

STRAWBERRY

(Fragaria virginiana) $2 \quad 0-18 \quad 29$

DANDELION

(Taraxacum officinale) 3

ARROWED LEAVED COLTSFOOT

(Petasites sagittatus)

2

$0-22 \quad 30$

$0-30 \quad 41$

GRASSES

AWNED SEDGE

(Carex atherodes)

$12 \quad 0-70 \quad 59$

MARSH REEDGRASS

(Calamagrostis canadensis)2

BEAKED SEDGE

(Carex rostrata)

WATER SEDGE

(Carex aquatilis)

$\begin{array}{lll}11 & 1-42 & 70 \\ 9 & 0-80 & 63\end{array}$

\section{ENVIRONMENTAL VARIABLES}

MOISTURE REGIME (MEAN):

SUBHYDRIC

NUTRIENT REGIME (MEAN):

RICH

ELEVATION:

576-606(588) M

SOIL DRAINAGE (MEAN):

POORLY

RANGELAND HEALTH RATING:

HEALTHY

\section{FORAGE PRODUCTION(KG/HA)}

GRASS

FORB

673(344-1002)

SHRUB

470(52-888)

TOTAL

$11(0-22)$

1169(448-1890)

ECOLOGICALLY SUSTAINABLE STOCKING RATE $0.8 \mathrm{HA} / \mathrm{AUM}(0.5 \mathrm{AUM} / \mathrm{AC})$ 


\section{DMA10a. Willow/Marsh reedgrass \\ (Salix spp./Calamagrostis canadensis)}

$\mathbf{n}=\mathbf{1 8}$ This community type is found along the edges of sedge and marsh reedgrass meadows and in moist depressions. Predominantly flat leaved willow becomes established at the edges of these meadows due to the shorter duration of standing water. Increased flooding and prolonged water logging may result in the disappearance of willow and a transition to a marsh reedgrass and water sedge meadow.

These sites are fairly productive but difficult to graze due to the moist ground conditions and heavy shrub cover which reduces access and mobility within the area.

\section{Plant Compositioncanopy Cover(\%)}

\section{SHRUBS}

BEBB WILLOW

FLAT LEAVED WILLOW

(Salix planifolia)

BASKET WILLOW

(Salix petiolaris)

MEAN RANGE CONST.
(Salix bebbiana)

\section{FORBS}

SKULLCAP

(Scutellaria galericulata) 1

MARSH HEMP NETTLE

(Stachys palustris)

STRAWBERRY

(Fragaria virginiana)

STINGING NETTLE

(Urtica dioica)

HORSETAIL

(Equisetum arvense)

$\begin{array}{lll}3 & 0-20 & 44 \\ 26 & 0-70 & 75 \\ 3 & 0-20 & 38\end{array}$

44

75

38

\section{ENVIRONMENTAL VARIABLES}

MOISTURE REGIME (MEAN):

SUBHYDRIC

NUTRIENT REGIME (MEAN):

$\mathrm{RICH}$

ELEVATION:

$606 \mathrm{M}$

SOIL DRAINAGE (MEAN):

POORLY

RANGELAND HEALTH RATING:

HEALTHY

\section{FORAGE PRODUCTION(KG/HA)}

$\begin{array}{ll}\text { GRASS } & 1325(900-1750) \\ \text { FORB } & 75(50-200) \\ \text { TOTAL } & 1400(950-1850)\end{array}$

ECOLOGICALLY SUSTAINABLE STOCKING RATE

\section{GRASSES}

AWNED SEDGE

(Carex atherodes)

MARSH REEDGRASS

(Calamagrostis canadensis)22

BEAKED SEDGE

(Carex rostrata)

FOWL BLUEGRASS

(Poa palustris)
$2 \quad 0-10 \quad 38$

$2 \quad 0-10 \quad 38$

\section{$0.7 \mathrm{HA} / \mathrm{AUM}(0.55 \mathrm{AUM} / \mathrm{AC})$}




\section{DMA11. Willow/Marsh reedgrass-Kentucky bluegrass \\ (Salix spp./Calamagrostis canadensis-Poa pratensis)}

$\mathbf{n = 6}$ This community type is very similar to the Willow/Marsh reedgrass community type, but has been heavily grazed favouring the growth of Kentucky bluegrass and dandelion. Continued heavy grazing pressure will eventually lead to a understory community that is similar to the Willow/Kentucky bluegrass/dandelion dominated community type.

\section{Plant COMPOSITION CANOPY COVER(\%)} MEAN RANGE CONST.

\section{SHRUBS}

WILLOW SPP.

(Salix spp.)

SNOWBERRY

(Symphoricarpos

occidentalis)

FORBS

MINT

(Mentha arvensis)

DANDELION

(Taraxacum offincinale) $\quad 15 \quad 1-41 \quad 100$

BUSHY CINQUEFOIL

(Potentilla paradoxa) $\quad 1 \quad 0-2 \quad 67$

GRASSES

MARSH REEDGRASS

(Calamagrostis canadensis)15 3-42 100

KENTUCKY BLUEGRASS

(Poa pratensis)

BALTIC RUSH

(Juncus balticus)

FOXTAIL BARLEY

(Hordeum jubatum)
$17 \quad 10-35 \quad 100$

$1 \quad 0-1 \quad 17$

$2 \quad 0-6 \quad 83$

$17 \quad 4-32 \quad 100$

$2 \quad 0-9 \quad 17$

$1 \quad 0-3 \quad 83$

\section{ENVIRONMENTAL VARIABLES}

MOISTURE REGIME (MEAN): SUBHYGRIC

NUTRIENT REGIME (MEAN): $\mathrm{RICH}$

ELEVATION: 600-606 M

SOIL DRAINAGE (MEAN): IMPERFECTLY

RANGELAND HEALTH RATING: HEALTHY WITH PROBLEMS

FORAGE PRODUCTION(KG/HA)

GRASS 2487(1800-1922)

FORB 1129(176-2450)

SHRUB $\quad 5(0-28)$

TOTAL 2487(1800-4250)

ECOLOGICALLY SUSTAINABLE STOCKING RATE $0.4 \mathrm{HA} / \mathrm{AUM}(1.0 \mathrm{AUM} / \mathrm{AC})$ 


\section{DMA12. Willow/Horsetail/Marsh reedgrass \\ (Salix spp./Equisetum arvensis/Calamagrostis canadensis)}

$\mathbf{n}=12$ This community type appears to be transitional between the horsetail (hygric/rich) and shrubby rich fen (subhydric/rich) ecosites described by Beckingham and Archibald (1996). It has plant species characteristic of both ecosites. This community type is also similar to the Willow-Alder/Fern community described on moist, nutrient rich seepage areas in the Lower Foothills subregion (Lane et al. 2000). This community type is very productive, but the high shrub cover and slope conditions make it difficult to graze. Horsetail the principal forage species is generally unpalatable to domestic livestock and can be poisonous to livestock in large amounts (Lodge et al. 1968). Consequently, this community type should be rated as secondary or non-use range.

\section{Plant COMPOSITION CANOPY COVER(\%) MEAN RANGE CONST.}

\section{SHRUBS}

SCOULER'S WILLOW

(Salix scouleriana)

WILLOW SPP.

(Salix spp.)

BRACTED HONEYSUCKLE

(Lonicera involcrata)

RED OSIER DOGWOOD

(Cornus stolonifera)

FORBS

STINGING NETTLE

(Urtica dioica)

COMMON HORSETAIL

$\begin{array}{llll}\text { (Equisetum arvensis) } & 15 & 1-60 & 100\end{array}$

LARGE LEAVED YELLOW AVENS

(Geum macrophyllum)

DEWBERRY

(Rubus pubescens)

GRASSES

MARSH REEDGRASS

(Calamagrostis canadensis) 22

$\begin{array}{lll}53 & 0-90 & 92 \\ 5 & 0-65 & 8 \\ 2 & 0-10 & 67 \\ 5 & 0-30 & 83\end{array}$

$9 \quad 0-60 \quad 58$

$2 \quad 0-10$

67

$0-97 \quad 75$

\section{ENVIRONMENTAL VARIABLES}

MOISTURE REGIME (MEAN): SUBHYGRIC

NUTRIENT REGIME (MEAN): PERMESOTROPHIC

\section{ELEVATION:}

$667 \mathrm{M}$

SOIL DRAINAGE (MEAN): MODERATELY WELL

RANGELAND HEALTH RATING: HEALTHY

\section{FORAGE PRODUCTION(KG/HA)}

$\begin{array}{ll}\text { GRASS } & 580 \\ \text { FORB } & 1272 \\ \text { TOTAL } & 1852\end{array}$

ECOLOGICALLY SUSTAINABLE STOCKING RATE NON-USE 


\section{DMA13. River alder/Horsetail (Alnus tenuifolia/Equisetum arvensis)}

$\mathbf{n}=6 \quad$ This community represents lowland sites surrounding open water or nutrient rich river flood plains This community is part of the red osier dogwood ecological site. Succession in the absence of disturbance will likely be to balsam poplar and eventually white spruce. The high shrub cover limits access to livestock, consequently, this community type would be considered non-use.

Plant COMPOSITION CANOPY COVER(\%) MEAN RANGE CONST.

TREES

PAPER BIRCH

(Betula papyrifera)

$4 \quad 0-25 \quad 33$

LARCH

(Larix laricina)

SHRUBS

WILLOW SPP.

(Salix spp.)

RIVER ALDER

(Alnus tenuifolia)

BRACTED HONEYSUCKLE

(Lonicera involcrata)

RED OSIER DOGWOOD

(Cornus stolonifera)

FORBS

DEWBERRY

(Rubus pubescens)

BISHOP'S CAP

(Mitella nuda)

HORSETAIL

(Equisetum arvensis) $\quad 6 \quad 0-27 \quad 67$

HEMP NETTLE

(Galeopsis tetrahit)

GRASSES

SEDGE

(Carex spp.)

MARSH REEDGRASS

(Calamagrostis canadensis)5

NODDING WOOD REED

(Cinna latifolia)

SMOOTH BROME

(Bromus inermis)
$0-50$

33

2

$1 \quad 0-5 \quad 17$

$2 \quad 1-3 \quad 100$

$43 \quad 10-90 \quad 100$

$2 \quad 0-7 \quad 33$

$0-3 \quad 50$

$0-30 \quad 67$

$0-5 \quad 50$

3

$0-3 \quad 50$

$0-10 \quad 83$

$3 \quad 0-20 \quad 33$

$\begin{array}{lll}8 & 0-50 \quad 17\end{array}$

\section{ENVIRONMENTAL VARIABLES}

MOISTURE REGIME (MEAN): HYGRIC

NUTRIENT REGIME (MEAN): RICH

ELEVATION: $606 \mathrm{M}$

SOIL DRAINAGE (MEAN): IMPERFECTLY

RANGELAND HEALTH RATING: HEALTHY

\section{FORAGE PRODUCTION(KG/HA)}

$\begin{array}{ll}\text { GRASS } & 102 \\ \text { FORB } & 330 \\ \text { SHRUB } & 104 \\ \text { TOTAL } & 536\end{array}$

ECOLOGICALLY SUSTAINABLE STOCKING RATE NON-USE 


\section{DMA14. Willow/Kentucky bluegrass/Dandelion (Salix spp./Poa pratensis/Taraxacum officinale)}

$\mathbf{n}=7 \quad$ This community type is very similar to the Willow/ Marsh reedgrass community type, but has been heavily grazed favouring the growth of Kentucky bluegrass and dandelion. Continued heavy grazing pressure eventually leads to a understory community that is dominated by Kentucky bluegrass and dandelion

\section{PlaANT Compositioncanopy Cover(\%)}

\section{SHRUBS}

WILLOW SPP.

(Salix spp.)

MEAN RANGE CONST.

SCOULER'S WILLOW

(Salix scouleriana)

FLAT LEAVED WILLOW

(Salix planifolia)

FORBS

MINT

(Mentha arvensis)

DANDELION

(Taraxacum officinale)

PLANTAIN

(Plantago major)

STRAWBERRY

(Fragaria virginiana)

GRASSES

MARSH REEDGRASS

(Calamagrostis canadensis)5

KENTUCKY BLUEGRASS

(Poa pratensis)

FOWL BLUEGRASS

(Poa palustris)

SMOOTH BROME

(Bromus inermis) $\begin{array}{lll}8 & 0-20 \quad 85\end{array}$

$9 \quad 0-50 \quad 29$

$11 \quad 0-40 \quad 29$

$2 \quad 0-10 \quad 57$

$32 \quad 0-80 \quad 71$

$1 \quad 0-5 \quad 21$

$2 \quad 0-10 \quad 57$

$0-10 \quad 86$

$10 \quad 0-40 \quad 43$

$4 \quad 0-10 \quad 71$

$4 \quad 0-30 \quad 14$

\section{ENVIRONMENTAL VARIABLES}

MOISTURE REGIME (MEAN): SUBHYGRIC

NUTRIENT REGIME (MEAN): $\mathrm{RICH}$

ELEVATION: 600-606 M

SOIL DRAINAGE (MEAN): IMPERFECTLY

RANGELAND HEALTH RATING: UNHEALTHY

\section{FORAGE PRODUCTION(KG/HA)}

GRASS $\quad 1100(700-1500)$

FORB $\quad 1250(750-1750$

TOTAL 2350(2250-2450)

ECOLOGICALLY SUSTAINABLE STOCKING RATE $0.4 \mathrm{HA} / \mathrm{AUM}(1.0 \mathrm{AUM} / \mathrm{AC})$ 


\section{DMA15. Sandbar willow-Yellow willow (Salix exigua-Salix lutea)}

$\mathbf{n = 1 4}$ This community type occurs on moist alluvial deposits which are adjacent to streams and rivers. This community can persist for some time if the site is subject to frequent flooding. However in the absence of disturbance it will eventually undergo succession to a spruce dominated community type. Thompson and Hansen (2002) described this community in the grassland natural region of Southern Alberta. They found that this community type disappeared as one moved north into the Parkland and it was replaced by basket willow and flat leaved willow dominated community types. Typically there is little understory vegetation found in this community type and it should be rated as non-use for livestock.

\section{PLANT COMPOSITION CANOPY COVER (\%)} MEAN RANGE CONST.

\section{TREES}

BALSAM POPLAR

(Populus balsamifera) $\quad 1 \quad 0-3 \quad 42$

\section{SHRUBS}

SANDBAR WILLOW
(Salix exigua)

YELLOW WILLOW

(Salix lutea)

SHINING WILLOW

(Salix lucida)

\section{FORBS}

HORSETAIL

(Equisetum arvense)

SILVERWEED

(Potentilla anserina)

PLANTAIN

(Plantago major)

$\begin{array}{lll}32 & 0-60 & 86 \\ 11 & 0-40 & 86 \\ 2 & 0-30 & 29\end{array}$

$12 \quad 0-90 \quad 64$

$2 \quad 0-10 \quad 43$

$2 \quad 0-20 \quad 29$

\section{GRAMINOIDS}

SMALL FRUITED BULRUSH

(Scirpus microcarpus)

KENTUCKY BLUEGRASS

(Poa pratensis)

SMOOTH BROME

(Bromus inermis)

2

2

$9 \quad 0-90$

\section{ENVIRONMENTAL VARIABLES}

MOISTURE REGIME: HYGRIC

NUTRIENT REGIME: RICH

ELEVATION: $600 \mathrm{M}$

SOIL DRAINAGE: IMPERFECTLY

RANGELAND HEALTH RATING: HEALTHY

Forage Production (KG/HA)

TOTAL $\quad 1000 *$ ESTIMATE

ECOLOGICALLY SUSTAINABLE STOCKING RATE NON-USE 


\section{DMA16: Bebb willow/Marsh reedgrass \\ (Salix bebbiana/Calamagrostis canadensis)}

$\mathbf{n = 1 3}$ This community type is found along the drier edges of marsh reedgrass meadows and in moist depressions and represents the transition between the flat leaved willow and basket willow dominated shrublands and the upland forest. Bebb willow is an upland species that prefers well drained sites. This species of willow is often found in the understory of aspen and balsam poplar dominated community types. Increased flooding and prolonged water logging may result in the disappearance of Bebb willow and favour the growth of flat leaved willow. In contrast the continued drying of the site will favour the growth of balsam poplar. These sites are fairly productive but difficul to graze due to the moist ground conditions and heavy shrub cover which reduces access and mobility within the area.

\section{PLANT COMPOSITION CANOPY COVER (\%)} Mean Range CONST.

\section{TREeS}

BALSAM POPLAR

(Populus balsamifera)

$2 \quad 0-10$

23

\section{SHRUBS}

BEBB WILLOW

(Salix bebbiana)

SNOWBERRY

(Symphoricarpos

occidentalis)

RASPBERRY

(Rubus idaeus)

ROSE

(Rosa acicularis)

\section{FORBS}

HORSETAIL

(Equisetum arvense)

DANDELION

(Taraxacum officinale)

STRAWBERRY

(Fragaria virginiana)

CANADA GOLDENROD

(Solidago canadensis)

$\begin{array}{lll}23 & 1-90 & 100 \\ 1 & 0-10 & 31 \\ 2 & 0-10 & 46 \\ 10 & 0-80 & 54\end{array}$

$\begin{array}{lll}4 & 0-20 \quad 69\end{array}$

$1 \quad 0-3 \quad 46$

$3 \quad 0-30 \quad 62$

$2 \quad 0-20 \quad 39$

\section{GRASSES}

KENTUCKY BLUE GRASS

(Poa pratensis)

SMOOTH BROME

(Bromus inermis)

SEDGE

(Carex spp.)

MARSH REEDGRASS

(Calamagrostis

canadensis)

\section{ENVIRONMENTAL VARIABLES}

MOISTURE REGIME: SUBHYGRIC-HYGRIC

NUTRIENT REGIME: RICH

ELEVATION(MEAN): $600 \mathrm{M}$

SOIL DRAINAGE: MOD. WELL

RANGELAND HEALTH RATING:

HEALTHY

\section{Forage Production (KG/Ha)}

TOTAL $1500 *$ ESTIMATE

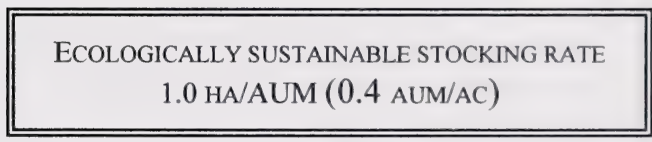




\section{DMA17: Red osier dogwood/Marsh reedgrass \\ (Cornus stolonifera/Calamagrostis canadensis)}

$\mathbf{n}=\mathbf{8}$ This community type was described on alluvial terraces, streambanks, abandoned channels on river floodplains and moist areas around springs and seeps. This community is much richer and has higher moisture levels than the adjacent upland aspen dominated forest, but it is much drier than the willow dominated shrublands in lower slope positions. In the absence of disturbance this community type will likely succeed to a balsam poplar and eventually white spruce dominated community type.

Livestock generally do not prefer this community type because of the dense nature of the understory, but heavy grazing pressure can reduce the understory cover and allow Kentucky bluegrass, timothy and smooth brome to invade.

\section{PlaAT COMPOSITION CANOPY COVER (\%)} MEAN RANGE CONST.

\section{TREES}

BALSAM POPLAR

(Populus balsamifera) $\quad 1 \quad 0-3 \quad 50$

\section{SHRUBS}

\section{RED OSIER DOGWOOD}

(Cornus stolonifera)

ROSE

(Rosa acicularis)

SNOWBERRY

(Symphoricarpos

occidentalis)

RASPBERRY

(Rubus idaeus)

$50 \quad 20-90 \quad 100$

$3 \quad 0-10 \quad 75$

$5 \quad 0-20 \quad 50$

$5 \quad 0-30 \quad 50$

\section{FORBS}

VEINY MEADOW RUE

(Thalictrum venulosum)

HORSETAIL

(Equisetum arvense)

AMERICAN VETCH

(Vicia americana)

$\begin{array}{lll}6 & 0-30 & 63 \\ 4 & 0-20 & 50 \\ 3 & 0-20 & 63\end{array}$

\section{GRASSES}

MARSH REEDGRASS

(Calamagrostis canadensis) $7 \quad 0-20 \quad 88$

FOWL BLUEGRASS

(Poa palustris)

$1 \quad 0-3 \quad 75$

\section{ENVIRONMENTAL VARIABLES}

MOISTURE REGIME: SUBHYGRIC

NUTRIENT REGIME : RICH

ELEVATION(MEAN): $600 \mathrm{M}$

SOIL DRAINAGE: MOD. WELL

RANGELAND HEALTH: HEALTHY

RANGELAND HEALTH RATING: HEALTHY

Forage Production (KG/HA)

TOTAL $1500 *$ ESTIMATED

ECOLOGICALLY SUSTAINABLE STOCKING RATE NON-USE 


\section{DMA18: Silverberry/Smooth brome \\ (Elaeagnus commutata/Bromus inermis)}

$\mathbf{n}=\mathbf{2}$ This community type has similar moisture and nutrient conditions to the previously described red osier dogwood dominated community type. Silverberry prefers moist, well drained seepage areas where overland flow provides additional moisture. This species can be found adjacent to streams and rivers, or seepage areas and snow accumulation areas adjacent to aspen stands. Thompson and Hansen (2002) found that these silverberry shrublands are often associated with disturbance in the grassland natural region of southern Alberta. Indeed, smooth brome is dominate in the understory of this community and it has likey invaded off the road allowance adjacent to this site. This community type is very productive because of the favourable moisture conditions, but as succession occurs to an aspen forest many of the palatable grass and forbs are often lost. This community will likely succeed to an $\mathrm{Pb} /$ Snowberry/Smooth brome dominated community type.

\section{PLANT COMPOSITION CANOPY COVER (\%) MEAN RANGE CONST.}

\section{TREES}

BALSAM POPLAR

(Populus balsamifera) $2 \quad 0-3 \quad 50$

\section{SHRUBS}

PRAIRIE ROSE

(Rosa arkansana)

SNOWBERRY

(Symphoricarpos

occidentalis)

SILVERBERRY

$\begin{array}{llll}\text { (Elaeagnus commutata) } & 65 & 50-80 & 100\end{array}$

\section{FORBS}

STINGING NETTLE

(Urtica dioica)

STRAWBERRY

(Fragaria virginiana) $\quad 5 \quad 0-10 \quad 50$

YARROW

(Achillea millefolium) $\quad 2 \quad 0-3 \quad 50$

\section{GRASSES}

KENTUCKY BLUE GRASS

(Poa pratensis)

SMOOTH BROME

(Bromus inermis)

QUACKGRASS

(Agropyron repens.)

$15 \quad 10-20 \quad 100$

$5 \quad 1-10 \quad 100$

$65 \quad 50-80 \quad 100$

$2 \quad 1-3 \quad 100$

ECOLOGICALLY SUSTAINABLE STOCKING RATE
TOTAL

$1500 *$ ESTIMATE

$1.0 \mathrm{HA} / \mathrm{AUM}(0.4 \mathrm{AUM} / \mathrm{AC})$

\section{ENVIRONMENTAL VARIABLES}

MOISTURE REGIME: SUBHYGRIC-MESIC

NUTRIENT REGIME: RICH

ELEVATION(MEAN): $600 \mathrm{M}$

SOIL DRAINAGE: WELL

RANGELAND HEALTH RATING:

HEALTHY WITH PROBLEMS

Forage Production (Kg/Ha) 


\section{DMA19: Bog willow \\ (Salix pedicellaris)}

$\mathbf{n}=4$ This community type was described on floating fens in the northern part of the Dry Mixedwood subregion near Gunn and Tulliby lake. Bog willow tends to prefer growing in swamps and fens throughout the Boreal forest of Northern Alberta (Johnson et al. 1995). The slight acidity on these sites limits productivity and these site are difficult to graze due to the moist ground conditions and heavy shrub cover which reduces access and mobility within the area. Consequently, this community should be rated as non-use.

\section{PLANT COMPOSITION CANOPY COVER (\%)} MEAN RANGE CONST.

\section{TREES}

PAPER BIRCH

(Betula papyrifera)

1

$0-1 \quad 50$

SHRUBS

BOG WILLOW

(Salix pedicellaris)

$\begin{array}{lll}70 & 50-90 & 100\end{array}$

FORBS

MARSH CINQUEFOIL

(Potentilla palustre)

SKULLCAP

(Scutellaria galericulata)

GRASSES

TWO STAMENED SEDGE

(Carex diandra)

WATER SEDGE

(Carex aquatilis)

NARROW REEDGRASS

(Calamagrostis stricta)

$8 \quad 0-20 \quad 75$

$15 \quad 0-40 \quad 75$

$13 \quad 0-50 \quad 50$

\section{ENVIRONMENTAL VARIABLES}

MOISTURE REGIME: SUBHYDRIC

NUTRIENT REGIME: MEDIUM

ELEVATION(MEAN): 600M

SOIL DRAINAGE: IMPERFECTLY

RANGELAND HEALTH RATING: HEALTHY

Forage Production (KG/HA)

TOTAL $\quad 1500 *$ ESTIMATE

ECOLOGICALLY SUSTAINABLE STOCKING RATE NON-USE 


\section{DMA20. Swamp horsetail \\ (Equisetum fluviatile)}

$\mathbf{n}=\mathbf{3} \quad$ This wetland community type is found near fresh water and is often associated with shallow water around lake shores or saturated wet spots in old river channels and sloughs. This community is often only found in small isolated spots or in narrow bands around the edge of lakes. As these areas dry, swamp horsetail is often replaced by sedge species. Swamp horsetail is generally unpalatable to livestock and the areas it grows in are often to wet for livestock to access. This community type should be rated as non-use.

\section{PLANT COMPOSITION CANOPY COVER(\%)}

\section{FORBS}

MEAN RANGE CONST.

SWAMP HORSETAIL

(Equisetum fluviatile)

MARSH WILLOW HERB

(Epilobium leptophyllum) $13 \quad 0-40 \quad 33$

SKULL CAP

(Scutellaria galericulata)

SMALL BEDSTRAW

(Galium trifidum)

$\begin{array}{lll}77 & 50-90 & 100 \\ 13 & 0-40 & 33 \\ 3 & 0-10 & 33 \\ 7 & 0-20 & 33\end{array}$

\section{GRASSES}

BEAKED SEDGE

(Carex rostrata)

WATER SEDGE

(Carex aquatilis)

CATTAIL

(Typha latifolia)
$7 \quad 0-20 \quad 33$

3

$0-10 \quad 33$

$8 \quad 0-20 \quad 66$

$1 \quad 0-1 \quad 33$

\section{ENVIRONMENTAL VARIABLES}

MOISTURE REGIME (MEAN): SUBHYDRIC-HYGRIC

NUTRIENT REGIME (MEAN): RICH

ELEVATION: $586(579-600) \mathrm{M}$

SOIL DRAINAGE (MEAN): POORLY TO VERY POORLY

RANGE HEALTH RATING:

HEALTHY

FORAGE PRODUCTION (KG/HA)

TOTAL 2000*ESTIMATE

ECOLOGICALLY SUSTAINABLE STOCKING RATE NON-USE 


\section{DMA21. Tall manna grass \\ (Glyceria grandis)}

$\mathbf{n}=\mathbf{3}$ This wetland community type is associated with the edge of the standing water of ponds, sloughs and slow meandering streams. As one moves away from the water to the drier edges the sedge meadow communities are found. This community is often only found in small isolated spots or in narrow bands around the edge of lakes. As these areas dry, tall manna grass is often replaced by sedge species. Tall manna grass is palatable to livestock, however, the areas it grows in are often to wet for livestock to access. This community type should be rated as non-use.

\section{Plant COMPOSITION CANOPY COVER(\%)}

$$
\text { MEAN RANGE CONST. }
$$

\section{FORBS}

SWAMP HORSETAIL

(Equisetum fluviatile)

MARSH WILLOWHERB

(Epilobium leptophyllum) 1

SMALL BEDSTRAW

(Galium trifidum)

\section{GRASSES}

TALL MANNA GRASS

(Glyceria grandis)

AWNED SEDGE

(Carex atherodes)

CATTAIL

(Typha latifolia)
$3 \quad 0-10 \quad 33$

$0-3 \quad 33$

$0-3 \quad 33$

$92 \quad 80-97 \quad 100$

$3 \quad 3-4 \quad 100$

$1 \quad 0-1 \quad 66$

\section{ENVIRONMENTAL VARIABLES}

MOISTURE REGIME (MEAN): SUBHYDRIC-HYGRIC

NUTRIENT REGIME (MEAN

PERMESOTROPHIC

ELEVATION:

$606 \mathrm{M}$

SOIL DRAINAGE (MEAN): VERY POORLY

RANGE HEALTH RATING: HEALTHY

FORAGE PRODUCTION (KG/HA)

GRASS 2000

TOTAL $2000 *$ ESTIMATE

ECOLOGICALLY SUSTAINABLE STOCKING RATE NON-USE 


\section{DMA22. Common reedgrass \\ (Phragmites australis)}

$\mathbf{n}=\mathbf{3}$ This community is found on the edges of shallow lakes and sloughs where the water table is near the surface for most of the growing season. Common reedgrass is common throughout the Boreal forest and this species is very important in binding the soil on river banks. The high sugar content of this plant makes it very palatable to livestock, but the moist ground conditions limits livestock use of these areas. This community type should be rated as non-use.

\section{Plant Composition Canopy COVER(\%)} MEAN RANGE CONST.

\section{SHRUBS}

WILLOW SPP.

(Salix spp.)

$$
2 \quad 0-5 \quad 33
$$

FORBS

MINT

(Mentha arvense)

SKULLCAP

(Scutellaria galericulata) 1

\section{GRASSES}

MARSH REEDGRASS

(Calamagrostis canadensis)1

COMMON REEDGRASS

(Phragmites australis)

AWNED SEDGE

(Carex atherodes)

CREEPING SPIKE RUSH

(Eleocharis palustris)

$\begin{array}{lll}58 & 13-80 & 100 \\ 2 & 0-5 & 66 \\ 3 & 0-10 & 33\end{array}$

\section{ENVIRONMENTAL VARIABLES}

MOISTURE REGIME (MEAN):

SUBHYDRIC

NUTRIENT REGIME (MEAN):

PERMESOTROPHIC

ELEVATION:

603(600-606)M

SOIL DRAINAGE (MEAN):

POORLY

RANGE HEALTH RATING:

HEALTHY

FORAGE PRODUCTION(KG/HA)
ECOLOGICALLY SUSTAINABLE STOCKING RATE

NON-USE 


\section{DMA23. Reed canary grass \\ (Phalaris arundinacea.)}

$\mathbf{n}=\mathbf{1}$ This community type is found along the edges of lakes, rivers, streams and pond margins. The European variety of this species has been widely distributed as a forage and often escapes from pastures and invades into the riparian and wetland areas, displacing more desirable species (Thompson and Hansen 2002). Once this species has invaded riparian areas it often forms monospecific stands because of its heavy sod forming habit (Thompson and Hansen 2002). Reed canary grass is moderately palatable to livestock and when it is grazed heavily the site often becomes invaded by thistle, dandelion and Kentucky bluegrass. This community type should be rated as secondary range and should be grazed in the spring when the canary grass is the most palatable.

\section{Plant COMPOSITION CANOPY COVER(\%)} MEAN RANGE CONST.

$\begin{array}{lccc}\begin{array}{l}\text { FORBS } \\ \text { CANADA THISTLE } \\ \text { (Cirsium arvense) }\end{array} & 20 & - & 100 \\ \begin{array}{l}\text { WATER SMARTWEED } \\ \text { (Polygonum amphibium) }\end{array} & 10 & - & 100 \\ \begin{array}{l}\text { SOW THISTL } \\ \text { (Sonchus spp.) }\end{array} & 3 & - & 100 \\ \begin{array}{l}\text { MARSH HEDGE-NETTLE } \\ \text { (Stachys palustris) }\end{array} & 3 & - & 100 \\ \begin{array}{l}\text { GRASSES } \\ \text { REDTOP } \\ \text { (Agrostis stolonifera) }\end{array} & 10 & - & 100 \\ \begin{array}{l}\text { REED CANARY GRASS } \\ \text { (Phalaris arundinacea) }\end{array} & 50 & - & 100 \\ \begin{array}{l}\text { SLENDER WHEATGRASS } \\ \text { (Agropyron trachycaulum) } 1\end{array} & - & 100\end{array}$

\section{ENVIRONMENTAL VARIABLES}

MOISTURE REGIME (MEAN): SUBHYDRIC

NUTRIENT REGIME (MEAN): PERMESOTROPHIC

ELEVATION:

$600 \mathrm{M}$

SOIL DRAINAGE: POORLY

RANGE HEALTH RATING: HEALTHY

FORAGE PRODUCTION (KG/HA)

TOTAL $2000 *$ ESTIMATE

ECOLOGICALLY SUSTAINABLE STOCKING RATE $0.5 \mathrm{HA} / \mathrm{AUM}$ 


\section{DMA24. Two stamened sedge}

\section{(Carex diandra)}

$\mathbf{n}=\mathbf{5}$ This community type was described in boggy areas adjacent to black spruce and larch dominated community types. Two stamened sedge tends to be found in the wetter areas where there is a floating mat of peat. As these areas dry out two stamened sedge will be replaced by willow, black spruce and larch species. Two stamened sedge is generally unpalatable to livestock and the areas it grows in are often too wet for livestock to access. This community type should be rated as non-use.

\section{Plant COMPOSITION Canopy COVER(\%)} MEAN RANGE CONST.

\section{SHRUBS}

BOG WILLOW

(Salix pedicellaris)

BOG BIRCH

(Betula glandulosa) $\quad 1 \quad 0-3 \quad 40$

FORBS

BUCK-BEAN

(Menyanthes trifoliata)

MARSH CINQUEFOIL

(Potentilla palustris)

MARSH MARIGOLD

(Caltha palustris)

GRASSES

TWO STAMENED SEDGE

(Carex diandra)

WATER SEDGE

(Carex aquatilis)

$\begin{array}{lll}6 & 0-30 & 40 \\ 1 & 0-3 & 40 \\ 7 & 0-20 & 60 \\ 5 & 0-10 & 60 \\ 3 & 0-10 & 40 \\ 82 & 60-90 & 100 \\ 1 & 0-3 & 40\end{array}$

\section{ENVIRONMENTAL VARIABLES}

MOISTURE REGIME (MEAN):

SUBHYDRIC

NUTRIENT REGIME (MEAN):

MESOTROPHIC

ELEVATION:

576-606(584) M

SOIL DRAINAGE (MEAN):

WELL

RANGE HEALTH RATING:

HEALTHY

FORAGE PRODUCTION (KG/HA)

TOTAL $1500 *$ ESTIMATE

ECOLOGICALLY SUSTAINABLE STOCKING RATE

NON-USE 


\section{DMA25. Rush meadow \\ (Juncus balticus, J. nodosus)}

$\mathbf{n}=2 \quad$ This community type was described on slightly saline sandy lakeshores. As the lake recedes rush species will invade into the sand of the lakeshore. Bailey et al. (1992) described rush dominated meadows in a saline sequence in the Yukon and Thompson and Hansen (2002) felt that rush dominated meadows were indicative of heavy grazing pressure in Southern Alberta. Rush species are generally unpalatable to livestock and these community types should be rated as non-use.

\section{PLANT COMPOSITION CANOPY COVER(\%)}

MEAN RANGE CONST.

TREES

BALSAM POPLAR

(Populus balsamifera)

\section{FORBS}

PRICKLY SOW THISTLE

(Sonchus asper)

GRASSES

BALTIC RUSH

(Juncus balticus)

KNOTTED RUSH

(Juncus nodosus)
1

0-1

50

2

0-3

50

$40 \quad 1-80$

100

$40 \quad 0-80 \quad 50$

\section{ENVIRONMENTAL VARIABLES}

MOISTURE REGIME (MEAN): SUBHYGRIC

NUTRIENT REGIME (MEAN): SUBMESOTROPHIC

ELEVATION:

$600 \mathrm{M}$

SOIL DRAINAGE (MEAN): MODERATELY WELL

RANGE HEALTH RATING: HEALTHY

FORAGE PRODUCTION (KG/HA)

TOTAL $1200 *$ ESTIMATE 


\section{DMA26. Creeping spike rush \\ (Eleocharis palustris)}

$\mathbf{n = 2}$ Thompson and Hansen (2002) described this type on somewhat alkaline sites in narrow bands along streams, rivers, lake margins and reservoirs. These sites are subject to yearly flooding. Typically these sites are almost pure stands of creeping spike rush. Creeping spike rush is generally unpalatable to livestock and the wet conditions limit livestock use. This community type should be rated as non-use.

\section{PLANT COMPOSITION CANOPY COVER(\%)} MEAN RANGE CONST.

\section{FORBS}

MARSH RAGWORT

(Senecio congestus)

SEASIDE BUTTERCUP

(Ranunculus cymbalaria) $10 \quad 0-20 \quad 50$

MARSH WILLOW HERB

(Epilobium palustre) $\quad 5 \quad 0-10 \quad 50$

COMMON BURREED

(Sparganium eurycarpum)5

GRASSES

CREEPING SPIKE RUSH

(Eleocharis palustris)

COMMON BULRUSH

(Scirpus acutus)

FOXTAIL BARLEY

(Hordeum jubatum)

$\begin{array}{lll}2 & 1-3 & 100 \\ 10 & 0-20 & 50 \\ 5 & 0-10 & 50 \\ 5 & 0-10 & 50\end{array}$

$60 \quad 50-70 \quad 100$

$5 \quad 0-10 \quad 50$

$2 \quad 0-3 \quad 50$

\section{ENVIRONMENTAL VARIABLES}

MOISTURE REGIME (MEAN):

$$
\text { SUBHYDRIC }
$$

NUTRIENT REGIME (MEAN):

ELEVATION:

$$
\text { PERMESOTROPHIC }
$$

$600 \mathrm{M}$

SOIL DRAINAGE (MEAN):

POORLY

RANGE HEALTH RATING:

HEALTHY

FORAGE PRODUCTION(KG/HA)

TOTAL $1200 *$ EsTIMATE

ECOLOGICALLY SUSTAINABLE STOCKING RATE NON-USE 


\section{DMA27. Three square rush \\ (Scirpus pungens)}

$\mathbf{n}=\mathbf{3}$ This community is an edge community forming dense stands along the edges of smaller streams, marshes and ponds. Three square rush is also tolerant of alkaline ( $\mathrm{pH} \mathrm{8.5)} \mathrm{and} \mathrm{saline} \mathrm{soils} \mathrm{(Thompson} \mathrm{and} \mathrm{Hansen} \mathrm{2002)} \mathrm{and}$ can be found adjacent to saline areas in conjunction with prairie bulrush in the southern part of the region. The palatability of this species is low to moderate. Consequently, three square rush communities are seldom grazed by livestock. This community should be rated non-use.

\section{PlaANT COMPOSITION CANOPY COVER(\%)}

MEAN RANGE CONST.

\section{SHRUBS}

SANDBAR WILLOW

(Salix exigua)

FORBS

SLENDER ARROW-GRASS

(Triglochin palustris)

SEASIDE BUTTERCUP

(Ranunculus cymbalaria) $2 \quad 0-3 \quad 66$

HORSETAIL

(Equisetum arvense) $\quad 3 \quad 0-10 \quad 33$

GRASSES

THREE SQUARE RUSH

(Scirpus pungens)

FOXTAIL BARLEY

(Hordeum jubatum)

NUTTALL'S SALTGRASS

(Puccinellia nuttalliana)

ROUGH HAIRGRASS

(Agrostis scabra)

$1 \quad 0-1 \quad 33$

$4 \quad 0-10 \quad 66$

$0-10 \quad 33$

$60 \quad 50-70 \quad 100$

$4 \quad 0-10 \quad 66$

$2 \quad 0-3 \quad 66$

$2 \quad 0-3 \quad 66$

\section{ENVIRONMENTAL VARIABLES}

MOISTURE REGIME (MEAN):

SUBHYGRIC

NUTRIENT REGIME (MEAN):

SUBMESOTROPHIC

ELEVATION:

$606 \mathrm{M}$

SOIL DRAINAGE (MEAN):

POORLY

RANGE HEALTH RATING:

HEALTHY

FORAGE PRODUCTION (KG/HA)

TOTAL $\quad 1200 *$ ESTIMATE

ECOLOGICALLY SUSTAINABLE STOCKING RATE

NON-USE 


\section{DMA28. Prairie bulrush \\ (Scirpus paludosus)}

$\mathbf{n}=\mathbf{2}$ This community type is often associated with alkaline and saline areas in semi-permanently flooded shallow edges of marshes and ponds (Thompson and Hansen 2002). Three square rush is often associated with the drier edges of this community type. The palatability of this species is low to moderate. Consequently, prairie bulrush communities are seldom grazed by livestock. This community should be rated non-use.

\section{PLANT COMPOSITION CANOPY COVER(\%)} MEAN RANGE CONST.

GRASSES

PRAIRIE BULRUSH

$\begin{array}{llll}\text { (Scirpus paludosus) } & 98 \quad-\quad 100\end{array}$

FOXTAIL BARLEY

$\begin{array}{llll}\text { (Hordeum jubatum) } & 1 & - & 100\end{array}$

NUTTALL'S SALTGRASS

$\begin{array}{llll}\text { (Puccinellia nuttalliana) } & 1 & - & 100\end{array}$

\section{ENVIRONMENTAL VARIABLES}

MOISTURE REGIME (MEAN):

SUBHYGRIC

NUTRIENT REGIME (MEAN):

SUBMESOTROPHIC

ELEVATION:

$600 \mathrm{M}$

SOIL DRAINAGE (MEAN):

POORLY

RANGE HEALTH RATING:

HEALTHY

FORAGE PRODUCTION(KG/HA)

TOTAL $1200 *$ ESTIMATE

ECOLOGICALLY SUSTAINABLE STOCKING RATE

SUGGESTED GRAZING CAPACITY

NON-USE 


\section{DMA29. Nuttall's saltgrass}

\section{(Puccinellia nuttalliana)}

$\mathbf{n}=\mathbf{2}$ This community is characteristic of saline and alkaline alluvial deposits adjacent to ponds, lake margins or seepage areas. This community type is fairly productive and heavy grazing will often lead to a community type dominated by foxtail barley. This community should be rated as secondary or primary range.

\section{PlaANT COMPOSITION CANOPY COVER(\%)}

\section{GRASSES}

MEAN RANGE CONST.

NUTTALL'S SALTGRASS

$\begin{array}{llll}\text { (Puccinellia nuttalliana) } & 97 & 97-98 & 100\end{array}$

PRAIRIE BULRUSH

(Scirpus paludosus)

FOXTAIL BARLEY

(Hordeum jubatum)

$2 \quad 0-3 \quad 50$

$1 \quad 0-1$

\section{ENVIRONMENTAL VARIABLES}

MOISTURE REGIME (MEAN): SUBHYGRIC

NUTRIENT REGIME (MEAN): SUBMESOTROPHIC

ELEVATION: $600 \mathrm{M}$

SOIL DRAINAGE (MEAN): MODERATELY WELL

RANGE HEALTH RATING: HEALTHY

FORAGE PRODUCTION(KG/HA)

TOTAL $\quad 1500 *$ ESTIMATE

ECOLOGICALLY SUSTAINABLE STOCKING RATE

$0.6 \mathrm{HA} / \mathrm{AUM}$ 


\section{DMA30. Foxtail barley \\ (Hordeum jubatum)}

$\mathbf{n}=\mathbf{2}$ This community represents a disturbance community. It can result from heavy grazing of tame pastures or native meadows in slightly saline areas. This community can also form on the edges of receding lake shores. As the lake drys foxtail barley will invade onto the drier edges. Foxtail barley is generally unpalatable to livestock and the seeds can get stuck in the animals mouth causing sores. Despite the high productivity of these sites they are often never used by livestock and should be rated as non-use.

\section{PLANT COMPOSITION CANOPY COVER(\%)} MEAN RANGE CONST.

\section{FORBS}

SEASIDE BUTTERCUP

(Ranunculus cymbalaria) $2 \quad 0-3 \quad 50$

DANDELION

(Taraxacum officinale) $\quad 1 \quad 0-1 \quad 50$

SEA SIDE ARROW-GRASS

(Triglochin maritima) $\quad 1 \quad 0-1 \quad 50$

GRASSES

FOXTAIL BARLEY

(Hordeum jubatum)

NUTTALL'S SALTGRASS

(Puccinellia nuttalliana)

CREEPING SPIKE RUSH

(Eleocharis palustris)

THREE SQUARE RUSH

(Scirpus pungens)

$\begin{array}{lll}1 & 0-1 & 50 \\ 80 & 80-81 & 100 \\ 1 & 1-2 & 100 \\ 5 & 0-10 & 50 \\ 2 & 0-3 & 50\end{array}$

\section{ENVIRONMENTAL VARIABLES}

MOISTURE REGIME (MEAN):

SUBHYGRIC

NUTRIENT REGIME (MEAN):

SUBMESOTROPHIC

ELEVATION:

$600 \mathrm{M}$

SOIL DRAINAGE (MEAN): IMPERFECTLY

RANGE HEALTH RATING: HEALTHY

FORAGE PRODUCTION(KG/HA)

TOTAL $1500 *$ ESTIMATE
ECOLOGICALLY SUSTAINABLE STOCKING RATE NON-USE 


\section{DRY MIXEDWOOD SUBREGION}

\section{TAME FORAGE COMMUNITIES}

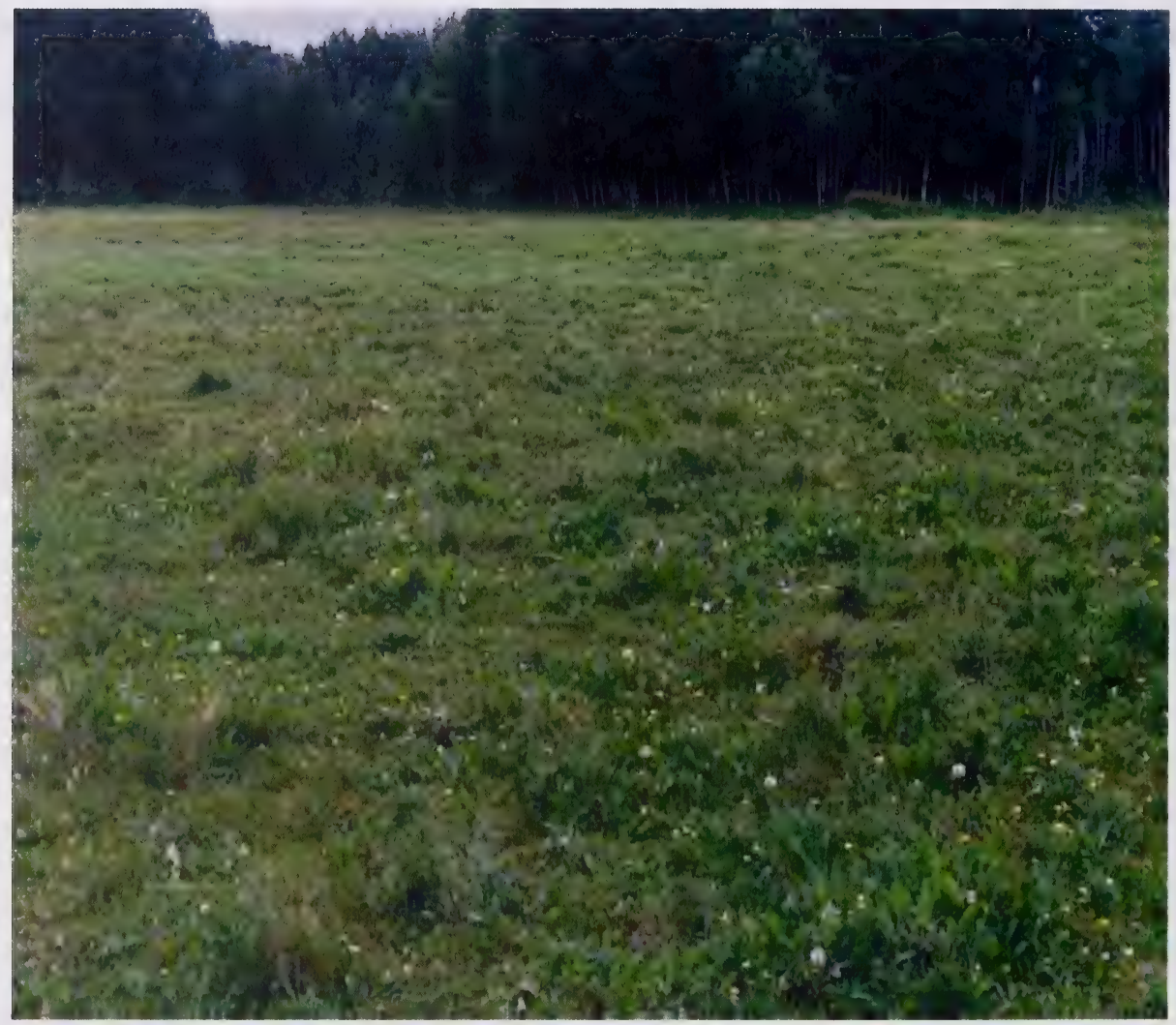

Photo 3. Typical Range improvement clearing in the Dry Mixedwood subregion 


\section{DM - TAME FORAGE COMMUNITIES}

(Cleared areas that have been broken and seeded to tame forage)

Throughout the Dry mixedwood subregion there are sites that have been deforested, broken, and seeded to tame forage. Usually these areas are mesic and moderately well to well drained with good nutrient levels. Because most of these tame forage stands are established on similar sites, the most influential factors affecting plant species composition are stand establishment and grazing regime.

Stand establishment is important because it determines what the initial plant species composition is going to be. Seed bed preparation and the type of seed sown are the two most important factors influencing stand establishment. Seed bed preparation is important because it helps to determine how well the sown seed germinates and establishes. If the seed bed is not well prepared the tame forage stand may establish poorly and native species can become a dominant component of the plant community.

After the stand is established, the grazing regime applied to the stand will determine the plant species composition. Generally, a light to moderate amount of grazing allows the stand to maintain itself while sustained heavy grazing causes the stand to degrade. Damage to a stand due to over grazing occurs more readily while the stand is establishing than it does when the stand is established. This is because the forage plants in an establishing stand have not had time to develop energy reserves in their roots, and are therefore, more susceptible to grazing induced damage.

Well distributed light to moderate grazing will normally maintain a forage stand similar to what was seeded on the site. These stands are generally the most productive and provide the best grazing opportunities for livestock. They are normally considered to be in good to excellent range health. Non use or very light grazing often results in the stand becoming dominated by the forage species that is most competitive under an ungrazed situation. Plant community changes which occur under heavy grazing are dependent on the grazing history (level of use, season of use and duration of the grazing regime). Overgrazed community types develop over a long period of repeated overgrazing. If weedy species such as Tall Buttercup or Canada thistle, become established on overgrazed sites, they can quickly become a dominant species.

We have organized the tame pasture communities within a moisture gradient dry (submesic), mesic and moist (subhygric). Within each moisture regime we have organized the communities along a grazing succession gradient. We have also identified successional sequences that occur in the absence of disturbance. These communities are often dominated by shrub and tree species. The successional diagram for tame pastures in the Dry Mixedwood subregion is outlined below. 
Successional sequences of tame pasture communities in the Dry Mixedwood subregion

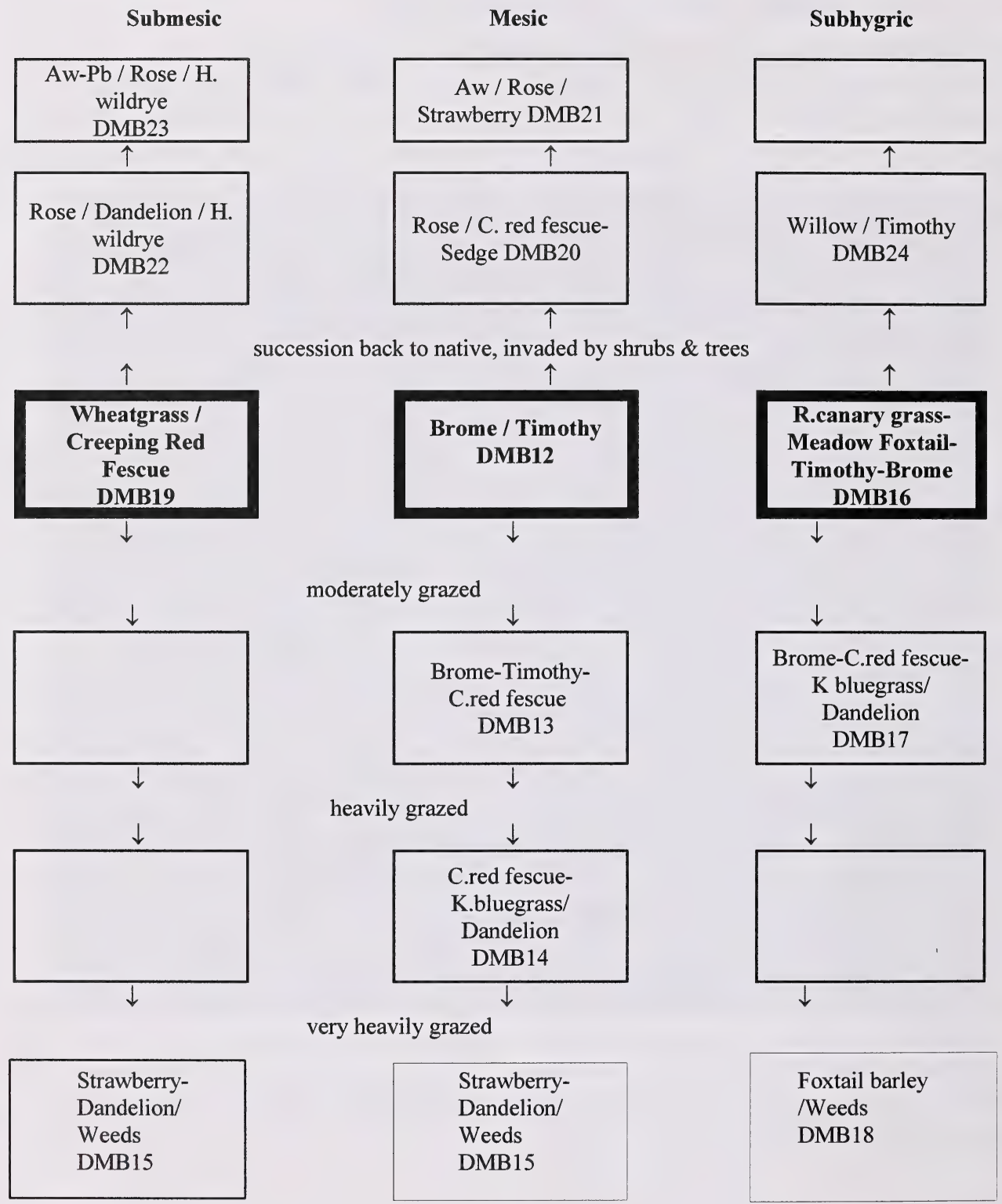




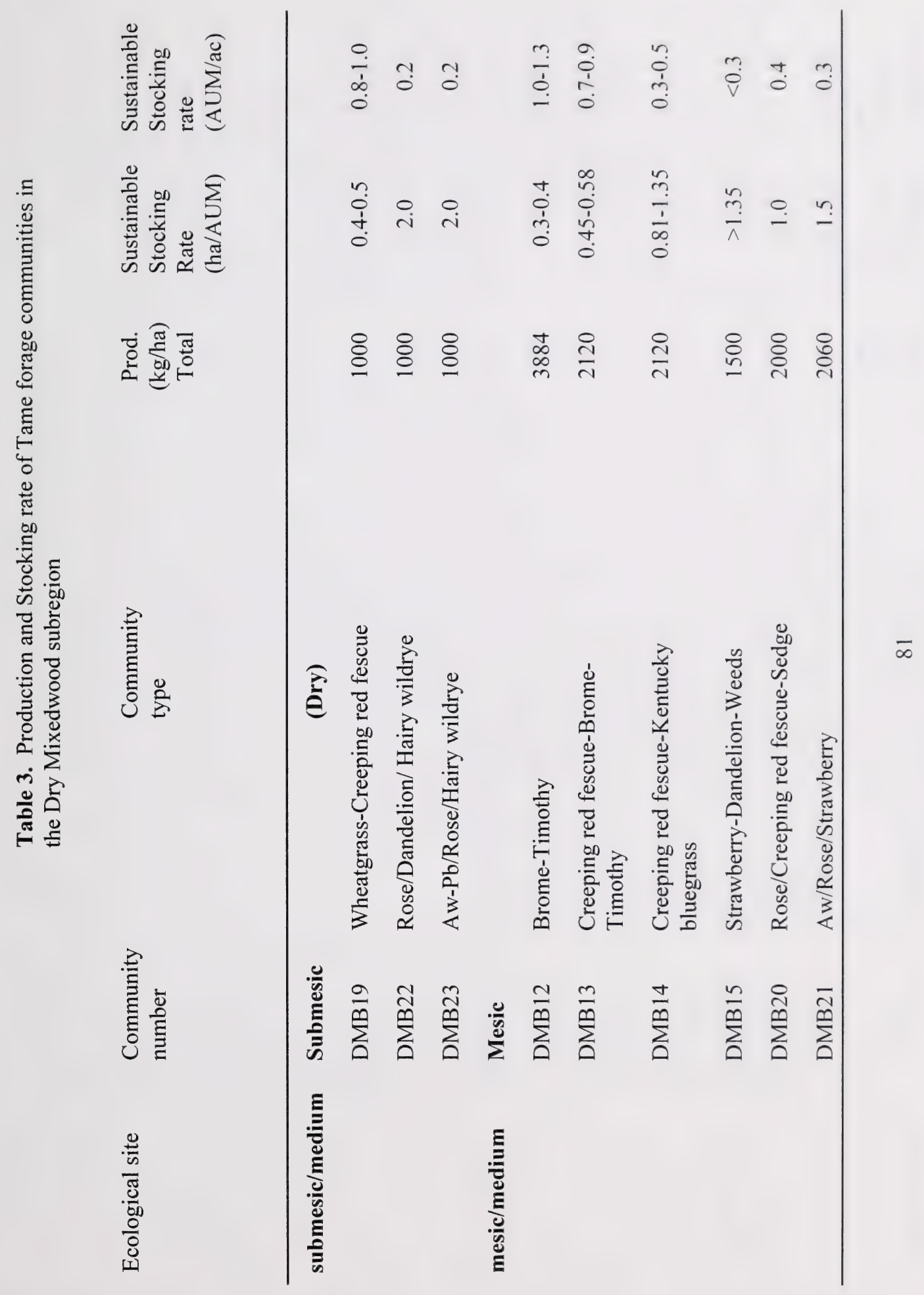




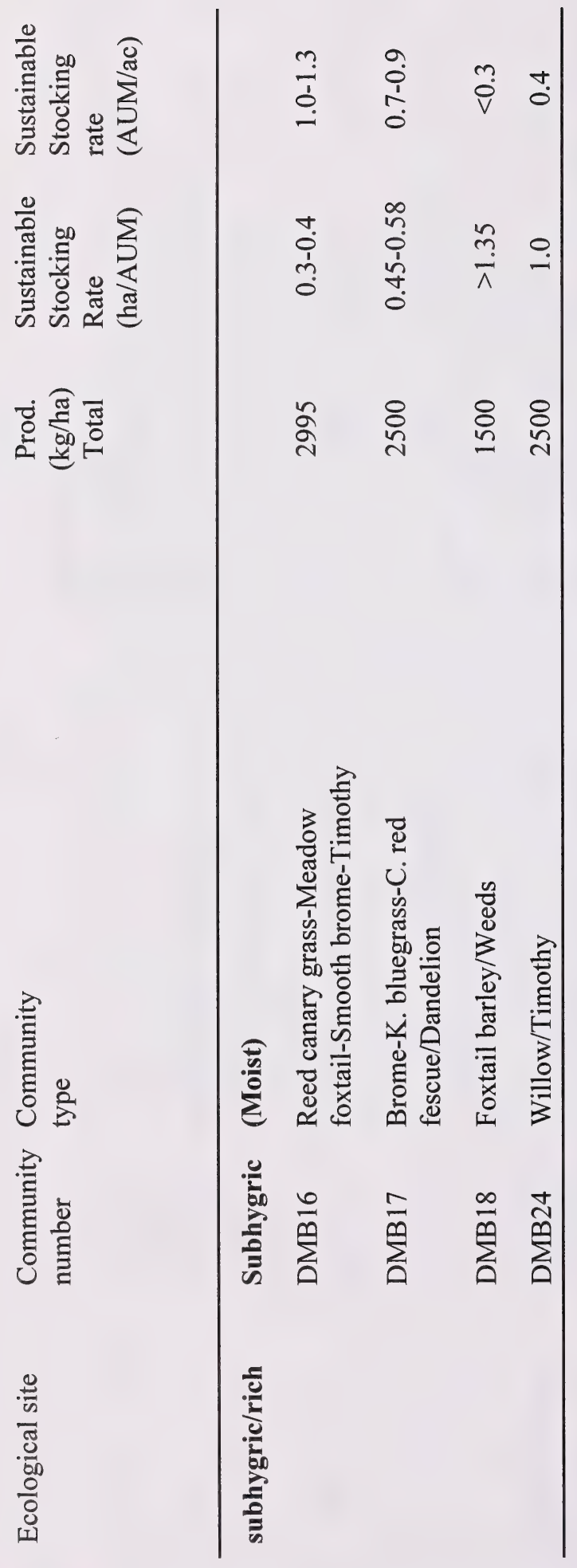

$\infty$ 


\section{Tame Grass Plant Communities - Dry Mixedwood Subregion}

1. Tame forage stand dominated by tall productive species............................. 2

Tame forage stand modified by grazing, aspen or shrub invasion..................... 4

2. Subhygric sites dominated by reed canary grass, meadow foxtail or timothy........

Reed Canary Grass / Meadow Foxtail / Timothy (DMB16)

Mesic or submesic sites dominated by smooth brome, meadow brome, wheatgrass, timothy or other tall, productive species........................................................................ 3

3. Submesic sites with wheatgrass and creeping red fescue.

Wheatgrass-Creeping Red Fescue (DMB19)

Mesic sites dominated by smooth brome, meadow brome, timothy or other tall, productive spp

....Brome / Timothy (DMB12)

4. Tame pasture invaded by aspen, balsam poplar or shrub species........................9 Species composition modified by grazing.........................................................5

5. Pasture moderately to heavily grazed; tall, productive spp. and grazing resistant spp codominate the site..........Creeping Red Fescue- Brome-Timothy(DMB13)

Pasture heavily to very heavily grazed; grazing resistant and / or weedy spp dominate

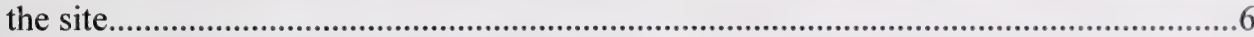

6. Pasture heavily grazed; grazing resistant spp dominate the site, dandelion, strawberry

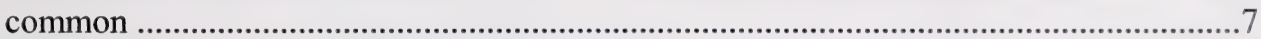

Pasture very heavily grazed; weedy invaders dominate the site............................... 8

7. Moist sites; dominated by grazing resistant spp.

... Brome-Creeping Red Fescue- Kentucky Bluegrass / Dandelion (DMB17)

Mesic sites; dominated by grazing resistant spp.

... Creeping Red Fescue / Kentucky Bluegrass / Dandelion (DMB14)

8. Mesic or submesic sites dominated by strawberry, dandelion, Canada thistle and other weedy spp .........Strawberry / Dandelion / Weeds (DMB15)

Subhygric sites dominated by foxtail barley, Canada thistle or other weedy spp.

Foxtail barley / Weeds (DMB18)

9. Old tame pastures with Aspen and Balsam Poplar invasion.

Newer tame pastures with shrub invasion, little tree growth

10. Mesic sites with strawberry Aw/Rose/Strawberry (DMB21)

Submesic sites with hairy wildrye Aw-Pb/Rose/Hairy wildrye (DMB23)

11. Submesic sites dominated by hairy wildrye and rose

..... Rose/Dandelion/Hairy wildrye (DMB22)

Mesic to subhygric sites.

12. Mesic sites with marsh reedgrass and sedge

Rose/Creeping red fescue-Sedge (DMB20)

Subhygric sites with willow invading.

Willow/Timothy (DMB24) 


\section{DMB12. Brome-Timothy \\ (Bromus inermis, B. biebersteinii-Phleum pratense)}

$\mathbf{n}=9$ This community type represents healthy condition tame pasture on mesic sites that were seeded with a timothy, smooth brome, meadow brome, creeping red fescue, alfalfa, clover mixture. Timothy establishes much quicker than creeping red fescue or smooth brome on pastures that have been recently seeded. Eventually creeping red fescue and smooth brome will outcompete timothy and this community will likely become dominated by creeping red fescue and smooth brome. Heavy to moderate grazing pressure will cause the tall growing grass species (Brome, timothy) to decline and allows low growing Kentucky bluegrass and dandelion to increase to form communities DMB13 and DMB14. Continued heavy grazing pressure will eventually lead to a community dominated by dandelion and weeds (DMB15). Light or no grazing or poor seed establishment will allow native trees, shrubs, forbs and grass to invade onto these sites to form communities DMB20 and 21.

\section{PLANT COMPOSITION CANOPY COVER(\%) MEAN RANGE CONST.}

SHRUBS

PRICKLY ROSE

(Rosa acicularis)

FORBS

CLOVER

(Trifolium spp.)

DANDELION

(Taraxacum officinale)

WILD STRAWBERRY

(Fragaria virginiana)

GRASSES

CREEPING RED FESCUE

(Festuca rubra)

TIMOTHY

(Phleum pratense)

KENTUCKY BLUEGRASS

(Poa pratensis)

SMOOTH BROME

(Bromus inermis)

MEADOW BROME

(Bromus biebersteinii)

$1 \quad 0-4$

22

$4 \quad 0-30$

44

$10 \quad 0-45$

67

$15 \quad 0-47 \quad 78$

7

8

8

0-35

78

$0-60 \quad 46$

$1 \quad 0-3 \quad 33$

49

25-77 100

$10 \quad 0-56 \quad 44$

\section{ENVIRONMENTAL VARIABLES}

MOISTURE REGIME (MEAN): MESIC-SUBHYGRIC

NUTRIENT REGIME (MEAN):
MEDIUM-RICH

ELEVATION:

457-606 (587)M

SOIL DRAINAGE (MEAN):

WELL TO MODERATELY WELL

RANGELAND HEALTH RATING:

HEALTHY

FORAGE PRODUCTION (KG/HA)

TOTAL 3884

ECOLOGICAL SUSTAINABLE STOCKING RATE

0.3-0.4 HA/AUM (1.0-1.3 AUM/AC) 


\section{DMB13. Creeping red fescue- Brome-Timothy \\ (Festuca-rubra-Bromus spp.-Phleum pratense)}

$\mathbf{n = 1 2}$ This community type develops on mesic sites that were seeded to a mixture of brome, timothy or other productive species with some grazing resistant species like creeping red fescue, A history of moderate to heavy grazing pressure results in a decline in the proportions of tall, productive species and an increase in the grazing resistant species. Heavy continuous grazing will allow Kentucky bluegrass and dandelion to invade into the stand to form a Kentucky bluegrass or Quackgrass/Dandelion dominated community type. This community type is usually considered to be in fair to good condition (healthy with problems).

\section{Plant Composition Canopy Cover(\%)}

TREES

MEAN RANGE CONST.

\section{ASPEN}

(Populus tremuloides)

SHRUBS

ROSE

(Rosa acicularis)

FORBS

Clover

(Trifolium spp.)

DANDELION

(Taraxacum officinale)

STRAWBERRY

(Fragaria virginiana)

GRASSES

CREEPING RED FESCUE

(Festuca rubra)

TIMOTHY

(Phleum pratense)

KENTUCKY BLUEGRASS

(Poa pratensis)

SMOOTH BROME

(Bromus inermis)

$\begin{array}{lll}2 & 0-10 & 33 \\ 2 & 0-5 & 75\end{array}$

$19 \quad 0-72 \quad 83$

$10 \quad 0-31 \quad 83$

$8 \quad 0-35 \quad 50$

$41 \quad 9-78 \quad 100$

$9 \quad 0-25 \quad 83$

$5 \quad 0-23 \quad 67$

$15 \quad 0-75 \quad 50$

\section{ENVIRONMENTAL VARIABLES}

MOISTURE REGIME (MEAN):

MESIC

NUTRIENT REGIME (MEAN):

MEDIUM

ELEVATION:

$609 \mathrm{M}$

SoIL DRAINAGE (MEAN):

WELL

RANGELAND HEALTH RATING:

HEALTHY WITH PROBLEMS

FORAGE PRODUCTION(KG/HA)

TOTAL 2120

ECOLOGICAL SUSTAINABLE STOCKING RATE $0.45-0.58$ HA/AUM $(0.7-0.9$ AUM/AC) 


\section{DMB14. Creeping red fescue-Kentucky bluegrass/Dandelion (Festuca rubra-Poa pratensis/ Taraxacum officinale)}

$\mathbf{n}=\mathbf{3 1}$ This community is representative of heavily grazed mesic sites and is dominated by grazing resistant species like Kentucky bluegrass, creeping red fescue or quackgrass. Heavy grazing tends to favour the growth of these low-growing or rhizomatuous species and that of weedy or disturbance induced species such as dandelion. These sites have poor health ratings and lower production than community types dominated by species like timothy and brome.

\section{PLANT COMPOSITION CANOPY COVER(\%)} MEAN RANGE CONST.

\section{SHRUBS}

RASPBERRY.

(Rubus idaeus.)

FORBS

ClOVER

(Trifolium spp.)

DANDELION

(Taraxacum officinale)

STRAWBERRY

(Fragaria virginiana)

GRASSES

CREEPING RED FESCUE

(Festuca rubra)

ТIMOTHY

(Phleum pratense)

SMOOTH BROME

(Bromus inermis)

KENTUCKY BLUEGRASS

(Poa pratensis)

QUACKGRASS

(Agropyron repens)
$1 \quad 0-30 \quad 25$

$13 \quad 0-45 \quad 100$

$21 \quad 0-42 \quad 91$

$2 \quad 0-4 \quad 72$

$15 \quad 0-75 \quad 40$

$3 \quad 0-13 \quad 53$

$2 \quad 0-3 \quad 25$

$15 \quad 0-36 \quad 78$

$5 \quad 0-45 \quad 20$

\section{ENVIRONMENTAL VARIABLES}

MOISTURE REGIME (MEAN): MESIC

NUTRIENT REGIME (MEAN): MEDIUM

ELEVATION:

576-701(658)M

SOIL DRAINAGE (MEAN):

WELL

RANGELAND HEALTH RATING:

UNHEALTHY

FORAGE PRODUCTION (KG/HA)

TOTAL 2120

ECOLOGICAL SUSTAINABLE STOCKING RATE 0.81-1.35 HA/AUM (0.3-0.5 AUM/AC) 


\section{DMB15. Strawberry-Dandelion-Weeds}

(Fragaria virginiana-Taraxacum officinale-Cirsium arvensis)

$\mathbf{n}=\mathbf{6}$ This community represents extremely heavily grazed mesic pasture sites. Generally, all that is left growing on these areas is dandelion. There also tends to be a lot of bare soil, which provides a place for noxious weeds (Canada thistle) to become established. This community would be rated unhealthy.

\section{Plant Composition Canopy Cover(\%)} MEAN RANGE CONST.

FORBS

ClOVER

(Trifolium spp.)

DANDELION

$\begin{array}{llll}\text { (Taraxacum officinale) } \quad 44 & 19-75 & 100\end{array}$

CANADA THISTLE

(Cirsium arvense)

GRASSES

TIMOTHY

(Phleum pratense)

CREEPING RED FESCUE

(Festuca rubra)

KENTUCKY BLUEGRASS

(Poa pratensis)

$\begin{array}{lll}1 & 0-6 & 50 \\ 44 & 19-75 & 100 \\ 5 & 0-29 & 33 \\ 2 & 0-7 & 67 \\ 1 & 0-1 & 67 \\ 17 & 3-74 & 100\end{array}$

\section{ENVIRONMENTAL VARIABLES}

MOISTURE REGIME (MEAN):

MESIC

NUTRIENT REGIME (MEAN):

MEDIUM

ELEVATION:

$455 \mathrm{M}$

SOIL DRAINAGE (MEAN):

WELL

RANGELAND HEALTH RATING:

UNHEALTHY

FORAGE PRODUCTION (KG/HA)

TOTAL 1500

ECOLOGICAL SUSTAINABLE STOCKING RATE

$>1.35 \mathrm{HA} / \mathrm{AUM}(<0.3 \mathrm{AUM} / \mathrm{AC})$ 


\section{DMB16. Reed canary grass-Meadow foxtail-Smooth brome-Timothy (Phalaris arundinacea-Alopecurus pratensis-Bromus inermis-Phleum pratense)}

$\mathbf{n}=\mathbf{2}$ This community type represents seeded areas on moist (subhygric) rich sites. Reed canary grass and meadow foxtail establish quickly in wet places that have been disturbed and will dominate very wet sites. Care should be taken when seeding reed canary grass. It appears that the commercial cultivars can be very invasive (Invasive plants of natural habitats 1992). In areas that have supported reed canary grass monocultures for extended periods many have seed banks devoid of other species. Meadow foxtail also seems particularly prone to increasing on moister grazed sites as it starts growth and heads out early. Meadow foxtail becomes unpalatable and is avoided by livestock if it is not grazed early enough in the spring.

Plant COMPOSITION CANOPY COVER(\%) MEAN RANGE CONST.

FORBS

AMERICAN VETCH

(Vicia americana)

DANDELION

(Taraxacum officinale)

STRAWBERRY

(Fragaria virginiana)

ClOVER

(Trifolium spp.)

GRASSES

REED CANARY GRASS

(Phalaris arundinacea)

TIMOTHY

(Phleum pratense)

SMOOTH BROME

(Bromus inermis)

CREEPING RED FESCUE

(Festuca rubra)

MEADOW FOXTAIL

(Alopecurus pratensis)

$\begin{array}{lll}1 & 0.1 & 50 \\ 3 & 2-3 & 100 \\ 13 & 0-26 & 50 \\ 15 & 6-25 & 100 \\ 28 & 0-55 & 50 \\ 5 & 2-7 & 100 \\ 21 & 0-41 & 50 \\ 6 & 1-11 & 100 \\ 11 & 0-22 & 50\end{array}$

\section{ENVIRONMENTAL VARIABLES}

MOISTURE REGIME (MEAN): SUBHYGRIC

NUTRIENT REGIME (MEAN): $\mathrm{RICH}$

ELEVATION: 579-606M

SOIL DRAINAGE (MEAN): WELL

RANGELAND HEALTH RATING:

HEALTHY

FORAGE PRODUCTION(KG/HA)

TOTAL 2995

ECOLOGICAL SUSTAINABLE STOCKING

RATE

0.3-0.4 HA/AUM (1.0-1.3 AUM/Ac) 


\section{DMB17. Brome-Creeping red fescue-Kentucky bluegrass/Dandelion (Bromus spp.-Festuca rubra-Poa pratensis/Taraxacum officinale)}

$\mathbf{n}=\mathbf{3}$ This community represents moderately grazed subhygric sites. Heavy continuous grazing will allow Kentucky bluegrass and dandelion to invade into the stand to form a Kentucky bluegrass or Quackgrass/Dandelion dominated community type. Continued heavy grazing pressure may eventually lead to site dominated by foxtail barley. This community type is usually considered to be in fair to good condition (healthy with problems).

\section{Plant COMPOSITION CANOPY COVER(\%)}

MEAN RANGE CONST.

\section{FORBS}

\section{ClOVER}

(Trifolium spp.) $\quad 19 \quad 6-31 \quad 100$

DANDELION

\section{(Taraxacum officinale)}

HORSETAIL

(Equisetum arvense)

STRAWBERRY

(Fragaria virginiana)

\section{GRASSES}

CREEPING RED FESCUE

(Festuca rubra)

MEADOW BROME

(Bromus biebersteinii)

SEDGE

(Carex spp.)

KENTUCKY BLUEGRASS

(Poa pratensis)

TIMOTHY

(Phleum pratense)

$29 \quad 15-38 \quad 100$

$4 \quad 2-6 \quad 100$

$2 \quad 1-2 \quad 100$

$62 \quad 40-80 \quad 100$

$21 \quad 17-23 \quad 100$

$16 \quad 1-45 \quad 100$

$5 \quad 1-10 \quad 100$

$\begin{array}{lll}3 & 2-4 & 100\end{array}$

\section{ENVIRONMENTAL VARIABLES}

MOISTURE REGIME (MEAN): SUBHYGRIC

NUTRIENT REGIME (MEAN): PERMESOTROPHIC

ELEVATION:

$$
667 \mathrm{M}
$$

SOIL DRAINAGE (MEAN): MODERATELY WELL

RANGELAND HEALTH RATING:

HEALTHY WITH PROBLEMS

FORAGE PRODUCTION(KG/HA)

TOTAL 2500

ECOLOGICAL SUSTAINABLE STOCKING RATE $0.45-0.58 \mathrm{HA} / \mathrm{AUM}(0.7-0.9 \mathrm{AUM} / \mathrm{AC})$ 


\section{DMB18. Foxtail barley/Weeds \\ (Hordeum jubatum/Cirsium arvensis)}

$\mathbf{n}=\mathbf{1}$ This community type develops on heavily grazed subhygric moist sites. This community was found in depressional areas and on river flood plains. Foxtail barley is also well adapted to growing on saline soils (Bailey et al. 1992). It is likely that the soils of this site are slightly saline. This community type would be considered nonuse because the principle forage species foxtail barley is generally unpalatable to livestock. Foxtail barley can also cause injury to livestock. The sharp seeds and awns may work their way into tongues, gums, eyes, noses or skins of animals.

PLANT COMPOSITION CANOPY COVER(\%) MEAN RANGE CONST.

\section{FORBS}

\section{ALFALFA}

(Medicago falcata.)

DANDELION

(Taraxacum officinale)

SWEET CLOVER

(Melilotus officinalis)

Clover

(Trifolium spp.)

GRASSES

FOXTAIL BARLEY

(Hordeum jubatum)

SMOOTH BROME

(Bromus inermis)

ТIMOTHY

(Phleum pratense)

FOWL BLUEGRASS

(Poa palustris)

MEAN RANGE CONST.

\section{ENVIRONMENTAL VARIABLES}

\author{
MOISTURE REGIME (MEAN): \\ SUBHYGRIC \\ NUTRIENT REGIME (MEAN): \\ RICH \\ ELEVATION: \\ 457-606(597)M
}

SOIL DRAINAGE (MEAN):

WELL

RANGELAND HEALTH RATING:

HEALTHY

FORAGE PRODUCTION(KG/HA)

TOTAL 1500

ECOLOGICAL SUSTAINABLE STOCKING RATE

$>1.35 \mathrm{HA} / \mathrm{AUM}(<0.3 \mathrm{AUM} / \mathrm{AC})$ 


\section{DMB19. Wheatgrass-Creeping red fescue-Timothy (Agropyron pectiniforme-Festuca rubra-Phleum pratense)}

$\mathbf{n}=\mathbf{1}$ This community type occurs on cleared pastures that were seeded on submesic (dry) sites in the eastern part of the subregion near St. Paul. These sites occur on very stoney well drained soils and it was thought crested wheatgrass would grow well in these site conditions. These pastures were seeded in the late 1980's with a mixture of pubscent wheatgrass, timothy, creeping red fescue, alfalfa, crested wheatgrass and sweet clover. Crested wheatgrass and creeping red fescue were found to dominate the dry hilltops and timothy was found on the moist lowland sites. There was little evidence of pubescent wheatgrass, alfalfa or sweet clover surviving from the original mix. These pastures often undergo succession to a shrub dominated community (DMB 11 ) and then a deciduous dominated community type (DMB9).

\section{Plant COMPOSITION CANOPY COVER(\%)} MEAN RANGE CONST.

SHRUBS

PRICKLY ROSE

(Rosa acicularis)

FORBS

Clover

(Trifolium spp.)

DANDELION

(Taraxacum offincinale) $29 \quad-\quad 100$

BEARBERRY

(Arctostaphylos uva-ursi)

GRASSES

CREEPING RED FESCUE

(Festuca rubra)

CRESTED WHEATGRASS

(Agropyron pectiniforme) $11 \quad-\quad 100$

TIMOTHY

(Phleum pratense)

KENTUCKY BLUEGRASS

(Poa pratensis)

$\begin{array}{lll}5 & - & 100 \\ 3 & - & 100\end{array}$

100

100

100

100

100

100

\section{ENVIRONMENTAL VARIABLES}

MOISTURE REGIME (MEAN):

SUBMESIC

NUTRIENT REGIME (MEAN):

MESOTROPHIC

ELEVATION:

$579 \mathrm{M}$

SOIL DRAINAGE (MEAN):

WELL

RANGELAND HEALTH RATING:

HEALTHY

FORAGE PRODUCTION(KG/HA)

TOTAL 1000

ECOLOGICAL SUSTAINABLE STOCKING RATE

0.4-0.5 HA/AUM (0.8-1.0 AUM/AC) 


\section{DMB20. Rose/Creeping red fescue-Sedge \\ (Rosa acicularis/Festuca rubra-Carex spp.)}

$\mathbf{n}=\mathbf{5} \quad$ As seeded pastures undergo succession back to a deciduous dominated forest they are often invaded by rose and willow before the trees become dominant. This community represents an early successional community of DMB21. Burning, cultivation and spraying with herbicide are all options that can be considered in order to control shrub regrowth. On mesic sites marsh reedgrass tends to be the native grass that invades. In contrast hairy wildrye will invade on drier sites.

Plant COMPOSITION CANOPY COVER(\%) MEAN RANGE CONST.

TREES

ASPEN

(Populus tremuloides)

6

0-15 40

SHRUBS

PRICKLY ROSE

(Rosa acicularis)

FORBS

Clover

(Trifolium spp.)

DANDELION

(Taraxacum officinale)

WILD STAWBERRY

(Fragaria virginiana)

GRASSES

CREEPING RED Fescue

(Festuca rubra)

TIMOTHY

(Phleum pratense)

KENTUCKY BLUEGRASS

(Poa pratensis)

HAIRY WILDRYE

(Elymus innovatus)

MARSH REEDGRASS

(Calamagrostis canadensis)3

SEDGE

(Carex spp.)
$7 \quad 0-14 \quad 80$

$2 \quad 1-7 \quad 100$

$1-23 \quad 100$

$19 \quad 0-64 \quad 80$

$4 \quad 0-12 \quad 60$

$2 \quad 0-7 \quad 60$

$1 \quad 0-3 \quad 40$

$0-13 \quad 20$

$0-24 \quad 80$
$12 \quad 1-25 \quad 100$

\section{ENVIRONMENTAL VARIABLES}

MOISTURE REGIME (MEAN):

MESIC

NUTRIENT REGIME (MEAN):

MEDIUM

ELEVATION:

603(600-606)M

SOIL DRAINAGE (MEAN):

WELL

RANGELAND HEALTH RATING:

HEALTHY WITH PROBLEMS

FORAGE PRODUCTION(KG/HA)

TOTAL 2000

ECOLOGICAL SUSTAINABLE STOCKING RATE

$1.0 \mathrm{HA} / \mathrm{AUM}(0.4 \mathrm{AUM} / \mathrm{AC})$ 


\section{DMB21. Aw/Rose/Strawberry \\ (Populus tremuloides/Rosa acicularis/Fragaria virginiana)}

$\mathbf{n}=\mathbf{5} \quad$ This community type occurs in mesic cultivated pastures that are being invaded by aspen. No grazing pressure or only light grazing pressure allows aspen to recolonize these cultivated pastures. Burning, cultivation and spraying with herbicide are all options that can be considered in order to control aspen regrowth.

Plant Composition Canopy Cover(\%) MEAN RANGE CONST.

\section{TREES}

ASPEN

(Populus tremuloides) $\quad 14 \quad 8-20 \quad 100$

BALSAM POPLAR

$\begin{array}{llll}\text { (Populus balsamifera) } & 1 & 0-1 & 40\end{array}$

SHRUBS

PRICKLY ROSE

(Rosa acicularis)

WILLOW

(Salix bebbiana)

SNOWBERRY

(Symphoricarpos

occidentalis)

FORBS

CLOVER

(Trifolium spp.)

DANDELION

(Taraxacum offincinale) $\quad 15 \quad 0-40 \quad 80$

WILD STAWBERRY

$\begin{array}{llll}\text { (Fragaria virginiana) } & 5 & 2-12 & 100\end{array}$

GRASSES

CREEPING RED FESCUE

$\begin{array}{llll}\text { (Festuca rubra) } & 2 & 0-5 & 40\end{array}$

TIMOTHY

KENTUCKY BLUEGRASS

(Poa pratensis)

HAIRY WILDRYE

(Elymus innovatus)

MARSH REEDGRASS

(Calamagrostis canadensis)1

$3 \quad 1-4 \quad 100$

$1 \quad 0-4 \quad 20$

$1 \quad 0-2 \quad 60$

$2 \quad 0-5 \quad 60$

$1 \quad 0-4 \quad 20$

$5 \quad 0-8 \quad 60$

$6 \quad 1-15 \quad 100$

$0-4 \quad 40$

\section{ENVIRONMENTAL VARIABLES}

MOISTURE REGIME (MEAN):

MESIC

NUTRIENT REGIME (MEAN):

MESOTROPHIC

ELEVATION:

$600 \mathrm{M}$

SOIL DRAINAGE (MEAN):

WELL

RANGELAND HEALTH RATING:

HEALTHY WITH PROBLEMS

FORAGE PRODUCTION(KG/HA)

TOTAL 2060

ECOLOGICAL SUSTAINABLE STOCKING RATE $1.5 \mathrm{HA} / \mathrm{AUM}(0.3 \mathrm{AUM} / \mathrm{AC})$ 


\section{DMB22. Rose/Dandelion/Hairy wildrye (Rosa acicularis/Taraxacum officinale/Elymus innovatus)}

$\mathbf{n}=\mathbf{1}$ This community represents early invasion of shrubs onto drier (submesic) sites on pastures in the St. Paul area of the subregion. As seeded pastures undergo succession back to a deciduous dominated forest they are often invaded by rose and willow before the trees become dominant. This community represents an early successional community of DMB23. Burning, cultivation and spraying with herbicide are all options that can be considered in order to control shrub regrowth.

\section{PLANT COMPOSITION CANOPY COVER(\%)} MEAN RANGE CONST.

TREES

ASPEN

$\begin{array}{llll}\text { (Populus tremuloides) } & 1 & - & 100\end{array}$

BALSAM POPLAR

(Populus balsamifera) $\quad 1 \quad-\quad 100$

SHRUBS

PRICKLY ROSE

(Rosa acicularis)

WILLOW

(Salix bebbiana)

SNOWBERRY

(Symphoricarpos

occidentalis)

FORBS

ClOVER

(Trifolium spp.)

DANDELION

(Taraxacum offincinale)

WILD STAWBERRY

(Fragaria virginiana)

GRASSES

CREEPING RED FESCUE

$\begin{array}{llll}\text { (Festuca rubra) } & 8 & - & 100\end{array}$

TIMOTHY

(Phleum pratense)

KENTUCKY BLUEGRASS

(Poa pratensis)

HAIRY WILDRYE

(Elymus innovatus)

CRESTED WHEATGRASS

(Agropyron pectiniforme)

\section{ENVIRONMENTAL VARIABLES}

MOISTURE REGIME (MEAN): SUBMESIC

NUTRIENT REGIME (MEAN): MEDIUM

ELEVATION:

$600 \mathrm{M}$

SOIL DRAINAGE (MEAN):

WELL

RANGELAND HEALTH RATING:

HEALTHY WITH PROBLEMS

\section{FORAGE PRODUCTION(KG/HA)}

TOTAL 1000

ECOLOGICAL SUSTAINABLE STOCKING.RATE $2.0 \mathrm{HA} / \mathrm{AUM}(0.2 \mathrm{AUM} / \mathrm{AC})$ 


\section{DMB23. Aw-Pb/Rose/Hairy wildrye \\ (Populus tremuloides-P. balsamifera/Rosa acicularis/Elymus innovatus)}

$\mathbf{n}=\mathbf{1}$ This community represents old pastures on dry sandy sites that were cleared of trees and aerial seeded with brome, timothy, crested wheatgrass and creeping red fescue in the 1980's near St. Paul. In the absence of disturbance these sites have been slowly encroached by trees and the understory has been invaded by hairy wildrye. These sites are moderately productive and are easily accessible to livestock. Consequently, they should be rated as primary or secondary range.

\section{PLANT COMPOSITION CANOPY COVER(\%)} MEAN RANGE CONST.

\section{TREES}

ASPEN

(Populus tremuloides)

BALSAM POPLAR

(Populus balsamifera)

SHRUBS

PRICKLY ROSE

(Rosa acicularis)

WILLOW

(Salix bebbiana)

SNOWBERRY

(Symphoricarpos

occidentalis)

FORBS

ClOVER

(Trifolium spp.)

DANDELION

(Taraxacum offincinale)

WILD STAWBERRY

(Fragaria virginiana)

GRASSES

CREEPING RED FESCUE

(Festuca rubra)

SLENDER WHEATGRASS

(Agropyron trachycaulum) 1

KENTUCKY BLUEGRASS

(Poa pratensis)

HAIRY WILDRYE

(Elymus innovatus)

CRESTED WHEATGRASS

(Agropyron pectiniforme) 1

\section{ENVIRONMENTAL VARIABLES}

MOISTURE REGIME (MEAN):

SUBMESIC

NUTRIENT REGIME (MEAN):

MEDIUM

ELEVATION:

$600 \mathrm{M}$

SOIL DRAINAGE (MEAN):

WELL

RANGELAND HEALTH RATING:

HEALTHY WITH PROBLEMS

100

100

100

FORAGE PRODUCTION(KG/HA)

TOTAL 1000

ECOLOGICAL SUSTAINABLE STOCKING RATE $2.0 \mathrm{HA} / \mathrm{AUM}(0.2 \mathrm{AUM} / \mathrm{AC})$ 


\section{DMB24. Willow/Timothy \\ (Salix spp./Phleum pratense)}

$\mathbf{n}=\mathbf{1}$ This community represents invasion of shrubs and trees onto tame pasture on moister sites. Willow favours growing on these moist, richer sites and will often invade off the edges of the pasture. Burning, cultivation and spraying with herbicide are all options that can be considered in order to control shrub regrowth.

Plant Composition Canopy COVER(\%) MEAN RANGE CONST.

SHRUBS

WILLOW

(Salix bebbiana)

$19 \quad 8-30 \quad 100$

FORBS

CLOVER

(Trifolium spp.)

DANDELION

$\begin{array}{llll}\text { (Taraxacum offincinale) } & 27 & 4-49 & 100\end{array}$

WILD STAWBERRY

$\begin{array}{llll}\text { (Fragaria virginiana) } & 6 & 0-12 & 100\end{array}$

HORSETAIL

(Equisetum arvense) $\quad 1 \quad 0-2 \quad 50$

GRASSES

TIMOTHY

(Phleum pratense)

SEDGE

(Carex spp.)

KENTUCKY BLUEGRASS

(Poa pratensis)

SMOOTH BROME

(Bromus inermis)

$3 \quad 2-3 \quad 100$

$0-12 \quad 100$

$0-2-50$

$45 \quad 43-46 \quad 100$

$5 \quad 0-9 \quad 50$

$1 \quad 0-1 \quad 50$

$2 \quad 0-3 \quad 50$

\section{ENVIRONMENTAL VARIABLES}

MOISTURE REGIME (MEAN): SUBHYGRIC

NUTRIENT REGIME (MEAN): PERMESOTROPHIC

ELEVATION:

$600 \mathrm{M}$

SOIL DRAINAGE (MEAN): WELL

RANGELAND HEALTH RATING: HEALTHY WITH PROBLEMS

FORAGE PRODUCTION(KG/HA)

TOTAL 2500

ECOLOGICAL SUSTAINABLE STOCKING RATE 1.0 $\mathrm{HA} / \mathrm{AUM}(0.4 \mathrm{AUM} / \mathrm{AC})$ 


\section{DRY MIXEDWOOD SUBREGION}

\section{DECIDUOUS FOREST COMMUNITY TYPES}

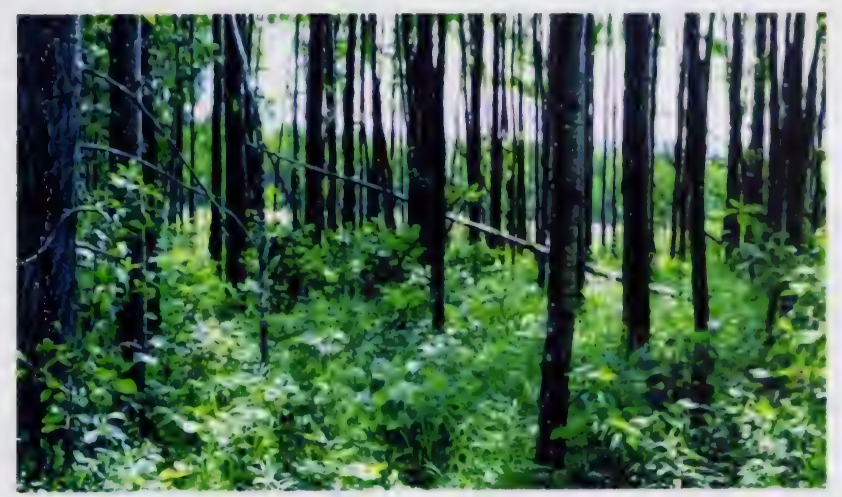

Photo 4. Aw/Rose/Tall forb community type in the Dry Mixedwood subregion

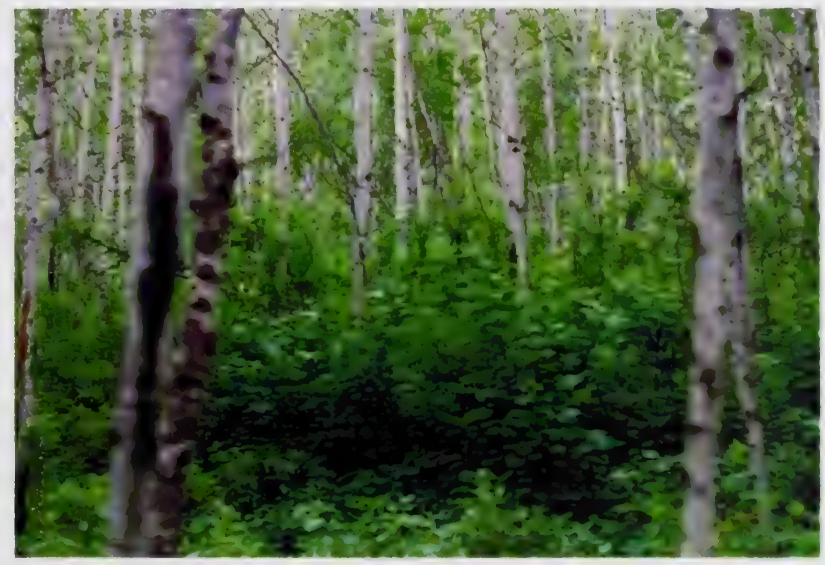

Phote 5. Aw/Hazelnut community is very common in the eastern ecodistricts of the Dry Mixedwood subregion 


\section{DM - DECIDUOUS FOREST COMMUNITIES}

All of the deciduous stands sampled in the Dry Mixedwood subregion were dominated by aspen and balsam poplar and had a significant rose understory. In both Brierley et al. (1985) and Beckingham's (1993) deciduous classifications in the same subregion rose was the dominant or codominant understory shrub species in nearly every aspen-dominated community type. In Beckingham's classification rose was present in 205 of the 209 aspen-dominated stands. Rose is well adapted to a wide variety of site conditions with a moderate supply of nutrients. The moisture regime can vary from submesic to subhygric and the sites can be well to imperfectly drained.

It appears the secondary forb and shrub species in association with rose characterize the ecological conditions of aspen forest types in the Dry Mixedwood subregion. Indeed, many of the deciduous types in Beckingham's classification were based on the secondary shrub species.

In the Dry Mixedwood 22 deciduous community types were described. The Aw/Blueberry type is found on well-drained, sandy sites in association with jack pine stands and the Aw/Dwarf bilberry/Bearberry/Mountain ricegrass community is found on slightly moister sites with loamy sand textures. The Aspen/Alder type is found on moist, moderately drained sites at higher elevations and the Aspen/Rose(Aw/Rose/Tall forb, Aw/Rose/Low forb, Aw/RoseHazelnut, Aw/Buffaloberry-Rose and Aw/Saskatoon-Rose) site types are moderately welldrained, with mesic moisture and mesotrophic nutrient regimes. Beckingham (1993), felt the Aspen/Buffaloberry type occurred on somewhat nutrient-poor soils. The Aspen/Rose/Tall and Low forb community types occupy similar site conditions. The difference between these two types may be related to grazing pressure. The Aspen/Rose/Low forb type has a low total cover of forbs (48\%), whereas the Aspen/Rose/Tall forb type has a high total cover of forbs (81\%). The increased grazing pressure in the Aspen/Rose/Low forb type may have caused a reduction in forb cover. The Aspen/Hazelnut type is found on mesic, well-drained sites and appears to be the reference deciduous type for this subregion, particularly in the more eastern ecodistricts. The hazelnut-dominated community types were very common within the eastern ecodistricts in the southern part of the subregion (St. Paul, Bonnyville, Smoky Lake). The presence of hazelnut appears to be indicative of warmer sites (Beckingham 1993) and have some fire history (Downing and Karpuk 1992).

A number of balsam poplar-dominated community types were described in the western and eastern ecodistricts. These communities are typical of forests situated along the flood plains of rivers and seepage areas in lower slope positions. The Balsam poplar-Aspen/Horsetail and Balsam poplar-Aspen/Willow type are found on moist poorly drained sites adjacent to some willow shrublands.

The sequence of the dominant community types in the landscape of the eastern ecodistricts is outlined in figure 5 . 


\section{Ecological site phase: d.1 low bush cranberry - Aw} DMC 10

$<N$

DMC 8a
timber harvest

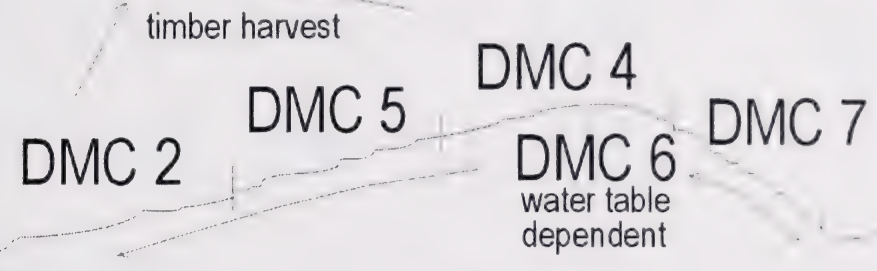

heavy grazing

DMC 3

sustained heavy grazing

$\mathrm{DMC} 3 \mathrm{a}$

NOTE: DMC 5 may not be present in eastern areas of the Dry Mixedwood subregion.

Figure 5. Overview of deciduous communities in the Dry Mixedwood subregion. 


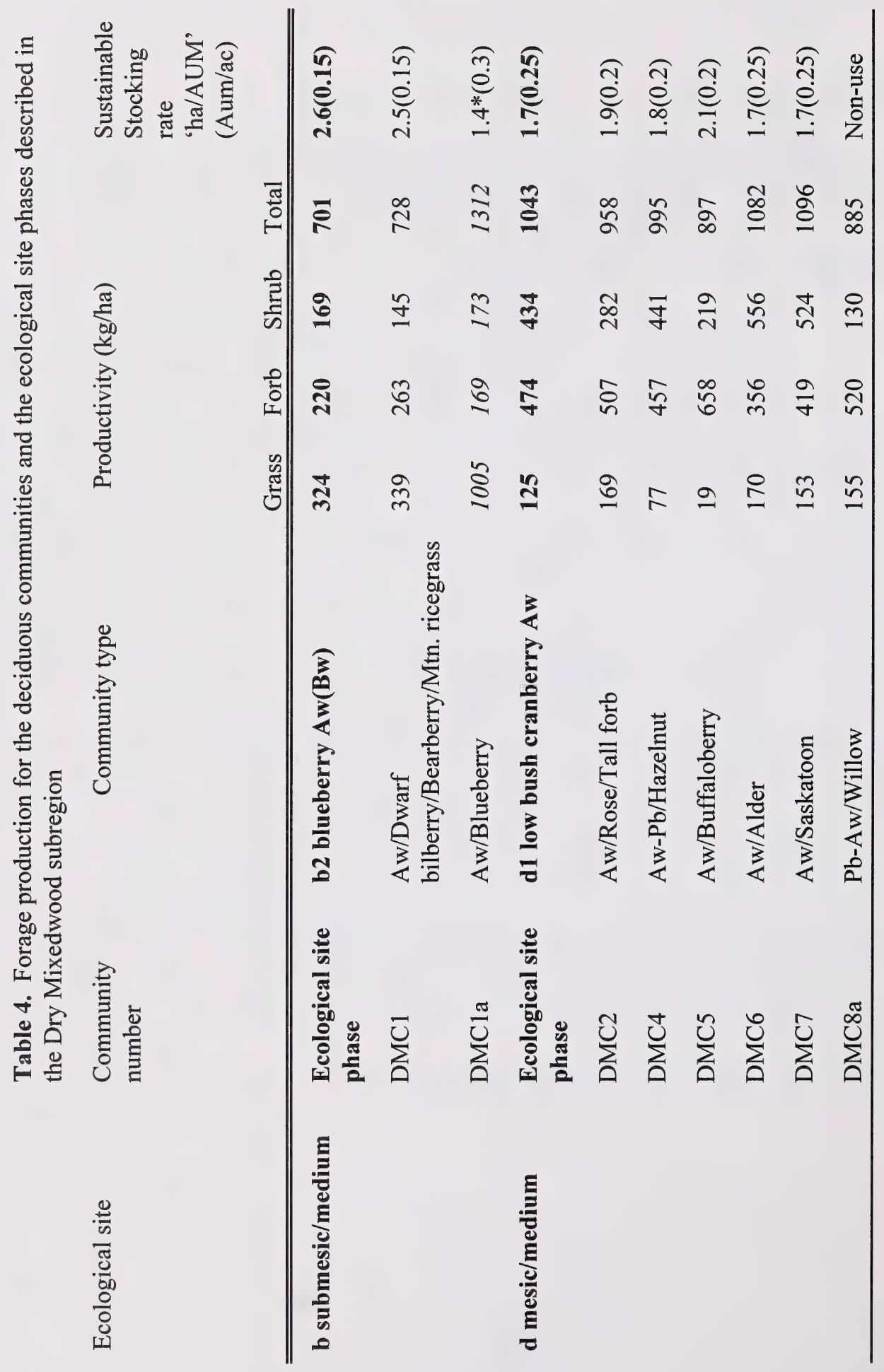

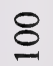




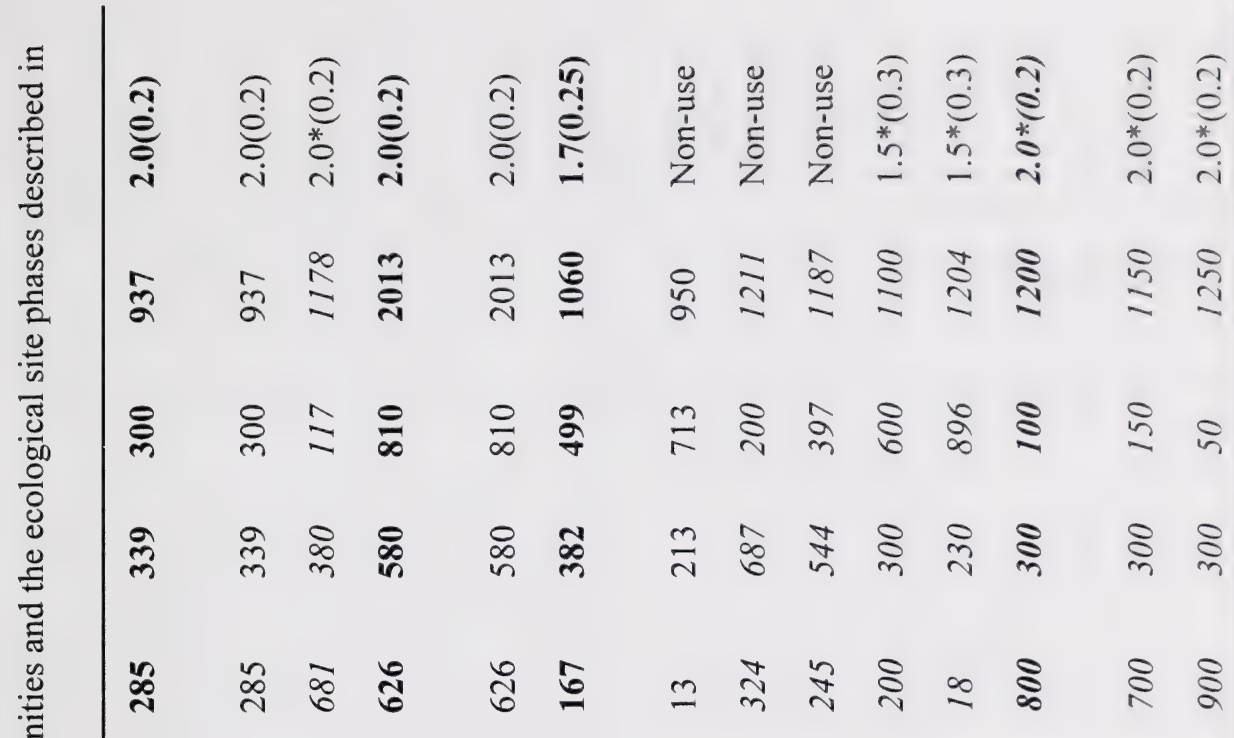

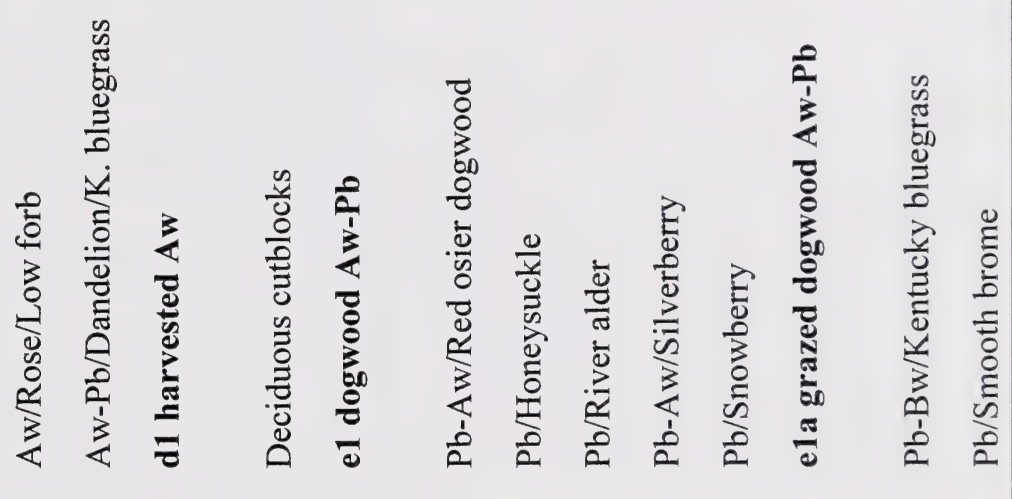




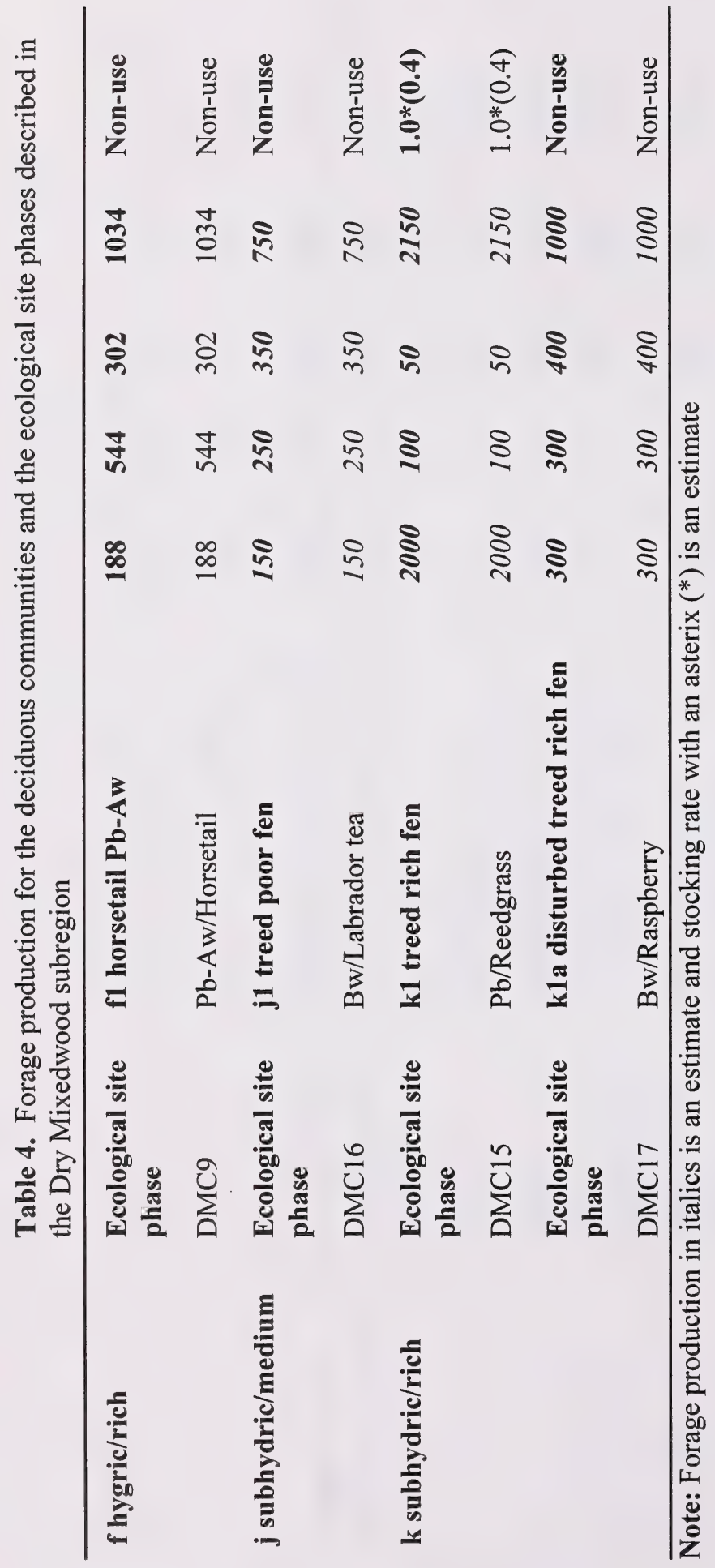




\section{Deciduous Community Types - Dry Mixedwood}

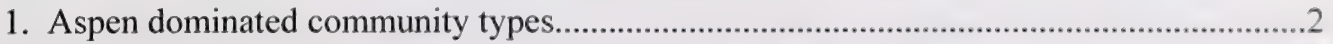

Balsam poplar and paper birch dominated community types........................................11

2. Dry sandy sites with blueberry and bearberry in understory............................................

Mesic sites dominated by alder, buffaloberry, saskatoon, rose, forbs and hazelnut (also includes grazed sites).

3. Sandy sites dominated by blueberry

DMC1a Aw/Blueberry

Loamy sand sites dominated by dwarf bilberry, bearberry and Mtn. ricegrass

DMC1 Aw/D.bilberry/Bearberry/Mtn. ricegrass

4. Grazed sites dominated by dandelion, Kentucky bluegrass or clover

DMC3a Aw/Dandelion/Kentucky bluegrass

Ungrazed sites dominated by alder, buffaloberry, saskatoon, hazelnut or rose................. 5

5. Beaked hazelnut dominates the understory............DMC4 Aw-Pb/Hazelnut

Sites dominated by alder, buffaloberry, rose, saskatoon (includes cutblocks).................6

6. Alder dominates the understory..................................DMC6 Aw/Alder

Buffaloberry, saskatoon, rose dominate understory (includes cutblocks)

7. Slope communities dominated by saskatoon......... DMC7 Aw/Saskatoon

Rose or buffaloberry dominated understory (includes cutblocks)...................................

8. Buffaloberry dominates the understory.....................DMC5 Aw/Buffaloberry

Rose and forb dominated understory (includes cutblocks).............................................

9. Deciduous cutblocks.

DMC10 Deciduous cutblocks

Rose, forb dominated understory.

10. Tall forb dominated (fireweed, showy aster, yellow peavine, wild sarsaparilla....

DMC2 Aw/Rose/Tall forb

Low forb dominated (bunchberry, twinflower, strawberry, wintergreen)

DMC3 Aw/Rose/Low forb

11. Ungrazed birch or balsam poplar dominated sites

Grazed birch or balsam poplar dominated sites (dandelion, Kentucky bluegrass) .......12

12. Smooth brome dominates understory.....

.DMC19 Pb/Smooth brome

Kentucky bluegrass and dandelion dominated.........DMC18 Pb/Dandelion/K. bluegrass

13. Balsam poplar dominated.

Paper birch dominated

14. Boggy area that has recently burned..............................MC16 Bw/Labrador tea

Wet sites with recent beaver activity....

DMC17 Bw/Raspberry

15. Very wet site, grass meadows invaded by balsam poplar...DMC15 Pb/Reedgrass

Upland sites dominated by red osier dogwood, river alder, snowberry, silverberry, horsetail or willow.

16. Riverine forests dominated by red osier dogwood in understory..DMC8 Pb-Aw/Red osier dogwood

Willow, river alder, snowberry, horsetail or silverberry dominates understory.

17. Willow dominates the understory...

DMC8a Pb-Aw/Willow

River alder, snowberry, horsetail or silverberry dominates understory.

18. Riverine forest dominated by river alder.

DMC12 Pb/River alder 
Snowberry, horsetail or silverberry dominates understory.....

19. Very moist sites dominated by horsetail in the understory..DMC9 Pb-Aw/Horsetail Snowberry or silverberry dominates understory .20

20. Snowberry dominates the understory DMC14 Pb/Snowberry Silverberry dominates the understory DMC13 Pb-Aw/Silverberry 


\section{DMC1. Aw/Dwarf bilberry/Bearberry/Mountain ricegrass \\ (Populus tremuloides/Vaccinium caespitosum/Arctostaphylos uva-ursi/Oryzopsis asperifolia)}

$\mathbf{n}=26$ This community type is found on dry, well-drained, loamy-sand sites and is part of the blueberry ecosite outlined by Beckingham and Archibald (1996). The canopy cover of aspen is open allowing for easy access by livestock, but the dry site conditions and poorer nutrient status limit the amount of regrowth after grazing. If this community type is managed for one rotation a year, it can contribute significantly to the overall carrying capacity of a lease. This community type would be considered to be primary range for domestic livestock.

Plant COMPOSITION CANOPY COVER(\%) MEAN RANGE CONST.

Trees

TREMBLING ASPEN

(Populus tremuloides)

WHITE SPRUCE

(Picea glauca)

43

20-75

100

$2 \quad 0-15 \quad 42$

SHRUBS

PRICKLY ROSE

(Rosa acicularis)

SASKATOON

(Amelanchier alnifolia)

BLUEBERRY

(Vaccinium myrtillus)

DWARF BILBERRY

(Vaccinium caespitosum) 4

FORBS

BEARBERRY

(Arctostaphylos uva-ursi)

TWINFLOWER

(Linnaea borealis)

LINDLEY'S ASTER

(Aster ciliolatus)

WILD LILY OF THE VALIEY

(Maianthemum canadense)5

YELLOW PEAVINE

(Lathyrus ochroleucus) 7

STRAWBERRY

(Fragaria virginiana)

GRASSES

MOUNTAIN RICE GRASS

(Oryzopsis asperfolia)

HAIRY WILDRYE

(Elymus innovatus)

PURPLE OATGRASS

(Schizachne purpurascens)3

NORTHERN RICEGRASS
(Oryzopsis pungens) $1 \quad 0-10 \quad 35$

\section{ENVIRONMENTAL VARIABLES}

MOISTURE REGIME:

SUBMESIC

NUTRIENT REGIME:

POOR

ELEVATION:

$455 \mathrm{M}$

SOIL DRAINAGE:

WELL

RANGELAND HEALTH RATING:

HEALTHY

FORAGE PRODUCTION(KG/HA)

GRASS 339(166-442)

FORBS 263(64-610)

SHRUBS $145(56-266)$

TOTAL $728(230-1284)$

ECOLOGICALLY SUSTAINABLE STOCKING

RATE

$2.5 \mathrm{HA} / \mathrm{AUM}(0.15 \mathrm{AUM} / \mathrm{AC})$ 


\section{DMC1a. Aw/Blueberry \\ (Populus tremuloides/Vaccinium myrtillus)}

$\mathbf{n = 1}$ This community type is found on dry, well-drained, sandy sites interspersed with stands of jack pine and is part of the blueberry ecosite outlined by Beckingham and Archibald (1996). The canopy cover of aspen is open allowing for easy access by livestock, but the dry site conditions and poorer nutrient status limit the amount of regrowth after grazing. If this community type is managed for one rotation a year, it can contribute significantly to the overall carrying capacity of a lease. This community type would be considered to be primary range for domestic livestock.

\section{PLANT COMPOSITION CANOPY COVER(\%) MEAN RANGE CONST.}

TREES

TREMBLING ASPEN

(Populus tremuloides) $\quad 35 \quad-\quad 100$

\section{SHRUBS}

PRICKLY ROSE

(Rosa acicularis)

LOW BUSH CRANBERRY

(Viburnum edule)

BLUEBERRY

(Vaccinium myrtillus)

100

100

100

\section{ENVIRONMENTAL VARIABLES}

\author{
MOISTURE REGIME: \\ SUBMESIC-SUBXERIC \\ NUTRIENT REGIME: \\ POOR \\ ELEVATION: \\ $455 \mathrm{M}$ \\ SOIL DRAINAGE: \\ WELL \\ RANGELAND HEALTH RATING: \\ HEALTHY
}

\section{FORBS}

BEARBERRY

(Arctostaphylos uva-ursi) $20 \quad-\quad 100$

TWINFLOWER

(Linnaea borealis) $\quad 5 \quad-\quad 100$

STRAWBERRY

100

$\begin{array}{llll}\text { (Fragaria virginiana) } & 5 & - & 100\end{array}$

WILD LILY OF THE VALLEY

(Maianthemum canadense)15 - 100

WILD SARSAPARILLA

(Aralia nudicaulis)

$7 \quad-\quad 100$

GRASSES

100

HAIRY WILDRYE

(Elymus innovatus)

NORTHERN RICEGRASS

(Oryzopsis pungens)

$5 \quad-\quad 100$

$4 \quad-\quad 100$

LICHEN

REINDEER LICHEN

(Cladina mitis)

$1 \quad-\quad 100$

\section{FORAGE PRODUCTION(KG/HA)}

GRASS 1005

FORBS 169

SHRUBS 138

TOTAL $1312 *$ ESTIMATE

ECOLOGICALLY SUSTAINABLE STOCKING RATE

$1.4 \mathrm{HA} / \mathrm{AUM}(0.3 \mathrm{AUM} / \mathrm{AC})$ 


\section{DMC2. Aw/Rose/Tall forb \\ (Populus tremuloides/Rosa acicularis/Tall forbs)}

$\mathbf{n}=\mathbf{7 1}$ This community type is part of the low bush cranberry ecosite outlined by Beckingham and Archibald (1996). This community type is also very similar to the Aspen/Rose/Low forb community type, but the cover of forbs is much higher. This appears to be related to the grazing pressure. The higher the grazing pressure on the Aw/Rose/Tall forb community type appears to cause a reduction in the cover of tall growing forbs (wild sarsaparilla. fireweed, peavine, showy aster) and favours the growth of low growing forbs (bunchberry, dewberry, wintergreen, strawberry). This community type is providing a moderate amount of forage for domestic livestock and would be considered primary range on a forested disposition.

\section{Plant COMPOSITION CANOPY COVER(\%)} MEAN RANGE CONST.

\section{TREES}

TREMBLING ASPEN

(Populus tremuloides)

BALSAM POPLAR

(Populus balsamifera) $\quad 4 \quad 0-10 \quad 58$

SHRUBS

HAZELNUT

(Corylus cornuta)

$2 \quad 0-12 \quad 34$

WILD RED RASPBERRY

(Rubus idaeus)

$4 \quad 0-10 \quad 83$

BRACTED HONEYSUCKLE

$\begin{array}{llll}\text { (Lonicera involucrata) } & 2 & 0-32 & 45\end{array}$

PRICKLY ROSE

(Rosa acicularis)

$12 \quad 0-24 \quad 91$

LOW BUSH CRANBERRY

(Viburnum edule)

$5 \quad 0-36 \quad 76$

FORBS

FIREWEED

(Epilobium angustifolium) 3

$0-7 \quad 61$

DEWBERRY OR RUNNING RASPBERRY

(Rubus pubescens) 4

\section{(Petasites palmatus) 2}

$0-7 \quad 87$

WILD STRAWBERRY

(Fragaria virginiana)

$2 \quad 0-10 \quad 78$

LINDLEY'S ASTER

(Aster ciliolatus)

YELLOW PEAVINE

(Lathyrus ochroleucus)

$\begin{array}{lll}3 \quad 0-4 & 87\end{array}$

WILD SARSAPARILLA

(Aralia nudicaulis)

GraSSES

MARSH REED GRASS

(Calamagrostis canadensis) 5

HAIRY WILDRYE
(Elymus innovatus)

3

0-30

70

\section{ENVIRONMENTAL VARIABLES}

MOISTURE REGIME:

MESIC TO SUBHYGRIC

NUTRIENT REGIME:

MEDIUM TO RICH

ELEVATION:

455-606(496) M

PERCENT SLOPE GRADIENT:

0 - 25(5)

SOIL DRAINAGE:

WELL TO MODERATELY WELL

RANGELAND HEALTH RATING:

HEALTHY

\section{FORAGE PRODUCTION(KG/HA)}

$\begin{array}{ll}\text { GRASS } & 169(0-444) \\ \text { FORBS } & 507(72-988) \\ \text { SHRUBS } & 282(118-378) \\ \text { TOTAL } & 958(624-1810)\end{array}$

\section{ECOLOGICALLY SUSTAINABLE STOCKING RATE \\ $1.9 \mathrm{HA} / \mathrm{AUM}(0.2 \mathrm{AUM} / \mathrm{AC})$}




\section{DMC3. Aw/Rose/Low forb \\ (Populus tremuloides/Rosa acicularis/Low forbs)}

$\mathbf{n}=\mathbf{6 0}$ This community type is part of the low bush cranberry ecosite described by Beckingham and Archibald (1996) and is very similar to the Aw/Rose/Tall forb community type previously described. The difference in the community types appears to be related to the grazing pressure. The higher the grazing pressure on the Aw/Rose/Tall forb community type appears to cause a reduction in the cover of tall growing forbs (wild sarsaparilla, fireweed, peavine, showy aster) and favours the growth of low growing forbs (bunchberry, dewberry, wintergreen, strawberry). This community type is providing a moderate amount of forage for domestic livestock and would be considered primary range on a forested disposition.

\section{Plant COMPOSITION CANOPY COVER(\%)}

MEAN RANGE CONST.

TREES

TREMBLING ASPEN

(Populus tremuloides)

BALSAM POPLAR

(Populus balsamifera)

$46 \quad 15-85 \quad 100$

SHRUBS

WILLOW SPP.

(Salix spp.)

SASKATOON

(Amelanchier alnifolia)

(Rubus idaeus)

SNOWBERRY

(Symphoricarpos

occidentalis)

PRICKLY ROSE

(Rosa acicularis)

FORBS

FIREWEED

(Epilobium angustifolium)

$\begin{array}{lcc}\text { DEWBERRY OR RUNNING RASPBERRY } \\ \text { (Rubus pubescens) } & 3 & 0-30\end{array}$

PALMATE-LEAVED COLTSFOOT

(Petasites palmatus)

(Fragaria virginiana)

LINDLEY's ASTER

(Aster ciliolatus)

BUNCHBERRY

(Cornus canadensis)

WILD SARSAPARILLA

(Aralia nudicaulis)

WINTERGREEN

(Pyrola asarifolia)

$1 \quad 0-30 \quad 53$

$3 \quad 0-12 \quad 92$

$2 \quad 0-12 \quad 82$

$\begin{array}{lll}5 & 0-22 \quad 83\end{array}$

$\begin{array}{lll}1 & 0-9 & 38\end{array}$

$3 \quad 0-20 \quad 85$

\section{GRASSES}

MARSH REED GRASS

(Calamagrostis canadensis)2 $\quad 0-20 \quad 77$

SLENDER WHEATGRASS

(Agropyron trachycaulum) $2 \quad 0-40 \quad 33$

HAIRY WILDRYE

$\begin{array}{llll}\text { (Elymus innovatus) } & 3 & 0-22 & 73\end{array}$

\section{ENVIRONMENTAL VARIABLES}

MOISTURE REGIME:

MESIC TO SUBHYGRIC

NUTRIENT REGIME:

MEDIUM TO RICH

ELEVATION:

455-697(524) M

PERCENT SLOPE GRADIENT:

0 - 5

SOIL DRAINAGE:

WELL TO MODERATELY WELL

RANGELAND HEALTH RATING:

HEALTHY WITH PROBLEMS

FORAGE PRODUCTION(KG/HA)

GRASS

285(12-996)

FORBS 339(90-842)

SHRUBS 300(0-896)

TOTAL 937(414-2074)

\section{ECOLOGICALLY SUSTAINABLE STOCKING RATE \\ $2.0 \mathrm{HA} / \mathrm{AUM}(0.2 \mathrm{AUM} / \mathrm{AC})$}




\section{DMC3a. Aw-Pb/Dandelion/Kentucky bluegrass \\ (Populus tremuloides-P. balsamifera/Taraxacum officinale/Poa pratensis )}

$\mathbf{n}=6$ This community represents the $\mathrm{Aw}$ or $\mathrm{Pb} / \mathrm{Rose} / \mathrm{Tall}$ forb community that has recieved prolonged heavy grazing. This community type often occurs in relatively small isolated patches created by intensive grazing adjacent to water, salt or temporary holding areas. The species richness and diversity of native shrubs, forbs, and grass is reduced and replaced by grazing resistant clover, dandelion and Kentucky bluegrass.

\section{Plant COMPOSITION Canopy COVER(\%)}

MEAN RANGE CONST.

\section{TREES}

TREMBLING ASPEN

(Populus tremuloides)

BALSAM POPLAR

$\begin{array}{llll}\text { (Populus balsamifera) } \quad 32 & 20-40 & 100\end{array}$

SHRUBS

WILLOW SPP.

(Salix spp.)

WILD RED RASPBERRY

(Rubus idaeus)

SNOWBERRY

(Symphoricarpos

occidentalis)

PRICKLY ROSE

(Rosa acicularis)

FORBS

ClOVER

(Trifolium spp.)

DEWBERRY OR RUNNING RASPBERRY

(Rubus pubescens)

DANDELION

(Taraxacum officinale)

WILD STRAWBERRY

$\begin{array}{llll}\text { (Fragaria virginiana) } & 3 & 1-3 & 100\end{array}$

SHOWY ASTER

(Aster conspicuus)

BUNCHBERRY

(Cornus canadensis) $\quad 1 \quad 0-1 \quad 50$

WILD SARSAPARILLA

(Aralia nudicaulis)

WINTERGREEN

(Pyrola asarifolia)

GRASSES

MARSH REED GRASS
(Calamagrostis canadensis) $1 \quad 0-3 \quad 67$

KENTUCKY BLUEGRASS

$\begin{array}{llll}\text { (Poa pratensis) } & 4 & 0-10 & 83\end{array}$

HAIRY WILDRYE

(Elymus innovatus) $\quad 2 \quad 0-10 \quad 50$

\section{ENVIRONMENTAL VARIABLES}

MOISTURE REGIME:

MESIC TO SUBHYGRIC

NUTRIENT REGIME:

MESOTROPHIC TO PERMESOTROPHIC

ELEVATION:

455-697(524) M

PERCENT SLOPE GRADIENT:

0 - 5

SOIL DRAINAGE:

WELL TO MODERATELY WELL

RANGELAND HEALTH RATING:

UNHEALTHY

\section{FORAGE PRODUCTION(KG/HA)}

GRASS $\quad 681$

FORBS $\quad 380$

SHRUBS $\quad 117$

TOTAL 1178*ESTIMATE

\section{ECOLOGICALLY SUSTAINABLE STOCKING RATE \\ 1.5 HA/AUM $(0.25$ AUM/AC)}




\section{DMC4. Aw-Pb/Hazelnut \\ (Populus tremuloides-P. balsamifera/Corylus cornuta)}

$\mathbf{n}=\mathbf{4 5}$ Beaked hazelnut is a common component of many of the deciduous stands in both the western and eastern ecodistricts of the Dry Mixedwood subregion. The presence of hazelnut appears to be indicative of warmer sites and have some fire history (Downing and Karpuk 1992). This community tends to occur on moderately to well drained, fine-textured and gently sloping till deposits. The total forage productivity of this community type is only moderate, but the majority of the production is coming from hazelnut, which is largely unpalatable to livestock at proper stocking levels. The high cover of hazelnut also restricts access to livestock, limiting the forage availability. This community type would be rated as secondary range.

\section{PLANT COMPOSITION CANOPY COVER(\%)} MEAN RANGE CONST.

TREES

TREMBLING ASPEN

(Populus tremuloides) $\quad 38 \quad 3-75 \quad 100$

BALSAM POPLAR

(Populus balsamifera) $5 \quad 0-60 \quad 38$

PAPER BIRCH

(Betula papyrifera) $20-70 \quad 4$

SHRUBS

HAZELNUT

(Corylus cornuta)

PRICKLY ROSE

(Rosa acicularis)

SNOWBERRY

(Symphoricarpos

occidentalis, albus)

SASKATOON

(Amelanchier alnifolia)

(Viburnum edule)

FORBS

LINDLEY'S ASTER

(Aster ciliolatus)

DEWBERRY OR RUNNING RASPBERRY

(Rubus pubescens)

PEAVINE

(Lathyrus ochroleucus) $\quad 5 \quad 1-10 \quad 100$

AMERICAN VETCH

(Vicia americana)

$39 \quad 12-70 \quad 100$

$9 \quad 0-25 \quad 82$

$4 \quad 4-10 \quad 100$

$4 \quad 0-18 \quad 89$

$3 \quad 0-16 \quad 71$

BUNCHBERRY

(Cornus canadensis)

WILD SARSAPARILLA

(Aralia nudicaulis)

GRASSES

MARSH REED GRASS

(Calamagrostis canadensis)4

\section{ENVIRONMENTAL VARIABLES}

MOISTURE REGIME:

MESIC TO SUBHYGRIC

NUTRIENT REGIME:

MEDIUM TO RICH

ELEVATION:

$455 \mathrm{M}$

PERCENT SLOPE GRADIENT:

$0-15 \%$

SOIL DRAINAGE:

WELL TO MODERATELY WELL

RANGELAND HEALTH RATING:

HEALTHY

FORAGE PRODUCTION(KG/HA)

GRASS 77(2-200)

FORBS 457(398-520)

SHRUBS 441(348-522)

TOTAL 995(830-1180)

ECOLOGICALLY SUSTAINABLE STOCKING

RATE

$1.8 \mathrm{HA} / \mathrm{AUM}(0.2 \mathrm{AUM} / \mathrm{AC})$ 


\section{DMC5. Aw/Buffaloberry \\ (Populus tremuloides/Shepherdia canadensis)}

$\mathbf{n = 5}$ This community type was found on mesic sites at higher elevations in the Saddle and Birch hills. Beckingham (1993) felt the Aw/Buffaloberry type was slightly drier and had a slightly poorer nutrient regime than the modal Aw/Rose community types. This type is providing a moderate amount of forage for domestic livestock, but the drier site conditions and poorer nutrient status will limit regrowth after grazing. Buffaloberry the predominant shrub species in this community type, is generally unpalatable to livestock.

\section{Plant COMPOSITION CANOPY COVER(\%)} MEAN RANGE CONST.

\section{TREES}

TREMBLING ASPEN

(Populus tremuloides) $\quad 52 \quad 30-85 \quad 100$

SHRUBS

BUFFALOBERRY

(Shepherdia canadensis) $25 \quad 11-38 \quad 100$

WILD RED RASPBERRY

(Rubus idaeus) $\quad 3 \quad 0-8$

BRACTED HONEYSUCKLE

(Lonicera involucrata) $\quad 1 \quad 0-2 \quad 20$

PRICKLY ROSE

$\begin{array}{llll}\text { (Rosa acicularis) } & 8 & 2-17 & 100\end{array}$

LOW BUSH CRANBERRY

(Viburnum edule) $\quad 3 \quad 0-14 \quad 40$

FORBS

BUNCHBERRY

(Cornus canadensis) $\quad 8 \quad 0-21 \quad 80$

DEWBERRY OR RUNNING RASPBERY

$\begin{array}{llll}\text { (Rubus pubescens) } & 2 & 0-9 & 60\end{array}$

YELLOW PEAVINE

$\begin{array}{llll}\text { (Lathyrus ochroleucus) } & 8 & 1-18 & 100\end{array}$

TWINFLOWER

(Linnaea borealis) $\quad 3 \quad 0-8 \quad 60$

GRASSES

MARSH REED GRASS

(Calamagrostis canadensis)2 $\quad 1-7 \quad 80$

HAIRY WILDRYE

$\begin{array}{llll}\text { (Elymus innovatus) } & 5 & 1-15 & 100\end{array}$

\section{ENVIRONMENTAL VARIABLES}

MOISTURE REGIME:

MESIC

NUTRIENT REGIME:

MEDIUM

ELEVATION:

455-758(556) M

PERCENT SLOPE GRADIENT:

$0-15$

SOIL DRAINAGE:

WELL

RANGELAND HEALTH RATING:

HEALTHY

\section{FORAGE PRODUCTION(KG/HA)}

GRASS $\quad$ 19(12-26)

FORBS 658(426-986)

SHRUBS 219(0-392)

TOTAL 897(840-1006)

\section{ECOLOGICALLY SUSTAINABLE STOCKING RATE \\ $2.1 \mathrm{HA} / \mathrm{AUM}(0.2 \mathrm{AUM} / \mathrm{AC})$}




\section{DMC6. Aw/Alder \\ (Populus tremuloides/Alnus crispa)}

$\mathrm{n}=7$ This community type was described at a higher elevation $(600 \mathrm{~m})$ in the Dry Mixedwood subregion. Brierly et al. (1985) and Beckingham (1993) both described aspen-alder communities at higher elevations $(>600 \mathrm{~m})$. It appears that the presence of alder may indicate a transition from the Dry Mixedwood into the Lower Foothills subregion.

This community type is providing a moderate amount of forage for domestic livestock, but the high cover of alder will limit access. Consequently, this community type should be rated as secondary range.

\section{Plant COMPOSITION CANOPY COVER(\%)}

MEAN RANGE CONST.

\section{TREES}

BALSAM POPLAR

(Populus balsamifera)

TREMBLING ASPEN

(Populus tremuloides) $\quad 45 \quad 25-60 \quad 100$

SHRUBS

PRICKLY ROSE

(Rosa acicularis)

WILD RED RASPBERRY

(Rubus idaeus)

LOW BUSH CRANBERRY

(Viburnum edule)

GREEN ALDER

(Alnus crispa)

FORBS

CREAM-COLOURED VETCHLING

(Lathyrus ochroleucus) 4

BUNCHBERRY

(Cornus canadensis) 12

STRAWBERRY

$\begin{array}{llll}\text { (Fragaria virginiana) } & 2 & 1-3 & 100\end{array}$

WILD SARSAPARILLA

(Aralia nudicaulis)

GRASSES

$20 \quad 5-40 \quad 100$

MARSH REEDGRASS

SLENDER WHEATGRASS

(Agropyron trachycaulum)2
$0-6 \quad 83$

$0-30 \quad 83$

100

$0-65 \quad 83$

$0-5 \quad 50$

\section{ENVIRONMENTAL VARIABLES}

MOISTURE REGIME:

MESIC

NUTRIENT REGIME:

MEDIUM

ELEVATION:

$600 \mathrm{M}$

PERCENT SLOPE GRADIENT:

$5 \%$

SOIL DRAINAGE:

WELL

RANGELAND HEALTH RATING:

HEALTHY

\section{FORAGE PRODUCTION(KG/HA)}

$\begin{array}{ll}\text { GRASS } & 170 \\ \text { FORBS } & 356 \\ \text { SHRUBS } & 556 \\ \text { TOTAL } & 1082\end{array}$

ECOLOGICALLY SUSTAINABLE STOCKING RATE

$1.7 \mathrm{HA} / \mathrm{AUM}(0.2 \mathrm{AUM} / \mathrm{AC})$ 


\section{DMC7. Aw/Saskatoon \\ (Populus tremuloides/Amelanchier alnifolia)}

$\mathbf{n}=9 \quad$ This community type is found on mesic, well drained south facing slopes that overlook rivers and creeks. Generally, hazelnut, chokecherry, saskatoon and snowberry are indicative of the Dry Mixedwood subregion and are usually found associated with each other. When saskatoon predominates it usually occurs on south and west facing slopes. Saskatoon provides important browse for wild ungulates. Livestock also find saskatoon palatable and in areas where there is extensive cattle grazing this species can be heavily browsed.

\section{PLANT COMPOSITION CANOPY COVER(\%)}

TREES

TREMBLING ASPEN

(Populus tremuloides)

BALSAM POPLAR

(Populus balsamifera)

SHRUBS

SASKATOON

(Amelanchier alnifolia)

PRICKLY ROSE

(Rosa acicularis)

WILD RED RASPBERRY

(Rubus idaeus)

SNOWBERRY

(Symphoricarpos

occidentalis)

CHOKECHERRY

(Prunus virginiana)

FORBS

MEAN RANGE CONST.

YELLOW PEAVINE

(Lathyrus ochroleucus) $\quad 2 \quad 0-10 \quad 78$

STRAWBERRY

(Fragaria virginiana) $\quad 1 \quad 0-10 \quad 78$

DEWBERRY OR RUNNING RASPBERRY

$\begin{array}{llll}\text { (Rubus pubescens) } & 2 & 0-10 & 67\end{array}$

WIID SARSAPARILLA

(Arailia nudicaulis)

$6 \quad 0-20$

89

GRASSES

MARSH REED GRASS

(Calamagrostis canadensis)3

$1-10$

\section{ENVIRONMENTAL VARIABLES}

MOISTURE REGIME:

MESIC

NUTRIENT REGIME: MEDIUM

ELEVATION: 455-630 M

PERCENT SLOPE GRADIENT:

$$
\text { 4(3-5\%) }
$$

ASPECT:

SOUTHERLY-WESTERLY

SOIL DRAINAGE:

WELL

RANGELAND HEALTH RATING:

HEALTHY

\section{FORAGE PRODUCTION(KG/HA)}

$\begin{array}{ll}\text { GRASS } & 153(42-264) \\ \text { FORBS } & 419(250-587) \\ \text { SHRUBS } & 524(514-534) \\ \text { TOTAL } & 1096(826-1365)\end{array}$

\begin{tabular}{|} 
ECOLOGICALLY SUSTAINABLE STOCKING \\
RATE \\
$1.7 \mathrm{HA} / \mathrm{AUM}(0.25 \mathrm{AUM} / \mathrm{AC})$ \\
\hline
\end{tabular} 


\section{DMC8. Pb-Aw/Red osier dogwood \\ (Populus balsamifera-P. tremuloides/Cornus stolonifera)}

$\mathbf{n = 5 1}$ This community type is typical of river floodplains throughout the Dry Mixedwood subregion. This community type tends to have a subhygric moisture and rich nutrient regime. Beckingham and Archibald (1996) found this community type on mid to lower slope topographic positions or near water courses where they recieve nutrient-rich seepage or flood waters for a portion of the growing season. This community type is one of the most productive in the Dry Mixedwood subregion, but the high cover of shrubs limits access to livestock. The high cover of tall growing shrubs (alder, red osier dogwood) also limits the growth of low shrubs, forbs and grass the principle forage species for domestic livestock in deciduous forests. As a result, this community should be rated as secondary or non-use range.

\section{PlaANT Composition Canopy Cover (\%) \\ MEAN RANGE CONST.}

TREES

BALSAM POPLAR

(Populus balsamifera)

TREMBLING ASPEN

(Populus tremuloides) $\quad 20 \quad 0-60 \quad 69$

PAPER BIRCH

(Betula papyrifera) $\quad 4 \quad 0-50 \quad 41$

SHRUBS

RED OSIER DOGWOOD

(Cornus stolonifera)

WILD RED RASPBERRY

(Rubus idaeus)

PRICKLY ROSE

(Rosa acicularis)

LOW BUSH CRANBERRY

(Viburnum edule)

FORBS

HORSETAIL

(Equisetum arvense)

WILD SARSAPARILLA

(Aralia nudicaulis)

PEAVINE

(Lathyrus ochroleucus)

FIREWEED

(Epilobium angustifolium) 3

GRASSES

MARSH REED GRASS

(Calamagrostis canadensis)4

$\begin{array}{lll}37 & 0-80 & 84 \\ 20 & 0-60 & 69 \\ 4 & 0-50 & 41\end{array}$

$23 \quad 8-70 \quad 100$

$4 \quad 0-18 \quad 60$

$\begin{array}{lll}9 & 0-18 & 78\end{array}$

$\begin{array}{lll}7 & 0-30 \quad 78\end{array}$

$4 \quad 0-10 \quad 73$

$7 \quad 0-40 \quad 80$

$0-4 \quad 57$

$0-20 \quad 61$

$0-50 \quad 75$

\section{ENVIRONMENTAL VARIABLES}

MOISTURE REGIME:

SUBHYGRIC

NUTRIENT REGIME:

RICH

ELEVATION:

455-606 M

SOIL DRAINAGE:

MODERATELY WELL

RANGELAND HEALTH RATING:

HEALTHY

FORAGE PRODUCTION(KG/HA)

GRASS 13(0-50)

FORBS 213(150-250)

SHRUBS 713(400-900)

TREE 13(0-50)

TOTAL 950(600-1150)

ECOLOGICALLY SUSTAINABLE STOCKING

RATE

NON-USE 


\section{DMC8a. Pb-Aw/Willow \\ (Populus balsamifera-P. tremuloides/Salix spp.)}

$\mathbf{n}=\mathbf{6} \quad$ This community type is typical of aspen forests adjacent to sloughs and wet meadows. The edges of the sedge meadows tend to be willow dominated. This community type represents the transition from the meadow edge into the aspen and balsam poplar dominated forest. This community type is relatively moist and nutrient rich. but the high cover of willow limits the light reaching the forest floor inhibiting the growth of understory shrub. forbs and grass. As a result there is little forage for domestic livestock. This community type would be rated as secondary or non-use range.

\section{Plant COMPOSITION CANOPY COVER(\%)} MEAN RANGE CONST.

\section{TREES}

BALSAM POPLAR

(Populus balsamifera)

TREMBLING ASPEN

(Populus tremuloides) $\quad 16 \quad 0-50 \quad 67$

PAPER BIRCH

$\begin{array}{llll}\text { (Betula papyrifera) } & 7 & 0-20 & 50\end{array}$

\section{SHRUBS}

WILLOW SPP.

(Salix spp.)

WILD RED RASPBERRY

(Rubus idaeus)

PRICKLY ROSE

BRACTED HONEYSUCKLE

(Lonicera involcrata)

FORBS

STRAWBERRY

(Fragaria virginiana)

LINDLEY'S ASTER

(Aster ciliolatus)

TALL LUNGWORT

(Mertensia paniculata) $\quad 4 \quad 1-20 \quad 100$

DEWBERRY

$\begin{array}{llll}\text { (Rubus pubescens) } & 2 & 1-4 & 100\end{array}$

HORSETAIL

(Equisetum arvense)

GRASSES

MARSH REED GRASS

(Calamagrostis canadensis)5

\section{ENVIRONMENTAL VARIABLES}

MOISTURE REGIME:

SUBHYGRIC

NUTRIENT REGIME:

$\mathrm{RICH}$

ELEVATION:

455-606 M

SOIL DRAINAGE:

MODERATELY WELL

RANGELAND HEALTH RATING:

HEALTHY

FORAGE PRODUCTION(KG/HA)

GRASS $\quad 130(0-260)$

FORBS 525(350-700)

SHRUBS 155(50-260)

TREE 75(0-150)

TOTAL 885(660-1110)

\section{ECOLOGICALLY SUSTAINABLE STOCKING RATE \\ NON-USE}




\section{DMC9. Pb-Aw/Horsetail}

\section{(Populus balsamifera-Populus tremuloides/Equisetum arvense)}

$\mathbf{n}=\mathbf{5}$ This community occupies lowland sites adjacent to black spruce and willow lowlands. It is very moist and nutrient-rich. Horsetail types in the other subregions also tend to be moister and richer than the modal Aw/Rose types. Past overgrazing pressure appears to have been heavy at one of the sites has resulted in an alteration of understory species composition and productivity. Overuse appears to lower species diversity and allows horsetail to increase in cover.

\section{PLANT COMPOSITION CANOPY COVER(\%)} MEAN RANGE CONST.

TREES

ASPEN

(Populus tremuloides)

BALSAM POPLAR

(Populus balsamifera)

SHRUBS

PRICKLY ROSE

(Rosa acicularis)

HONEYSUCKLE

(Lonicera involcrata)

RED OSIER DOGWOOD

(Cornus stolonifera)

FORBS

HORSETAIL

$\begin{array}{llll}\text { (Equisetum arvense) } & 30 & 5-60 & 100\end{array}$

BUNCHBERRY

(Cornus canadensis) $\quad 1 \quad 0-6 \quad 40$

TALL LUNGWORT

(Mertensia paniculata)

DEWBERRY

(Rubus pubescens)

VEINY MEADOW RUE

(Thalictrum venulosum)

BISHOP's CAP

(Mitella nuda)

STRAWBERRY

(Fragaria virginiana)

GRASSES

MARSH REED GRASS

(Calamagrostis canadensis) 2
$25 \quad 0-85 \quad 60$

$41 \quad 0-75 \quad 80$

$3 \quad 1-10 \quad 100$

$0-16 \quad 60$

$0-8 \quad 60$

$0-7 \quad 60$

$0-5 \quad 80$

$0-4 \quad 20$

$0-3 \quad 40$

$0-2 \quad 60$

$0-5 \quad 80$

\section{ENVIRONMENTAL VARIABLES}

MOISTURE REGIME:

HYGRIC

NUTRIENT REGIME:

$\mathrm{RICH}$

ELEVATION:

590-667 M

SOIL DRAINAGE:

IMPERFECTLY

RANGELAND HEALTH RATING:

HEALTHY

\section{FORAGE PRODUCTION(KG/HA)}

$\begin{array}{ll}\text { GRASS } & 188(104-272) \\ \text { FORBS } & 544(450-638) \\ \text { SHRUBS } & 302(0-604) \\ \text { TOTAL } & 1034(910-1158)\end{array}$

ECOLOGICALLY SUSTAINABLE STOCKING

RATE

NON-USE 


\section{DMC10. Deciduous cutblocks and unseeded clearings \\ (Populus tremuloides)}

$\mathbf{n}=\mathbf{4}$ This community type represents deciduous cutblocks and clearings that have not been seeded to tame forage species. Marsh reedgrass and strawberry initially dominated these areas. As succession occurs an understory of aspen and rose predominate. As the tree cover increases the understory species structure and diversity declines. Initially these clearings are very productive for domestic livestock until the trees grow back and limit accessiblity. Care should be taken when grazing these cutblocks that the trees are not damaged and there is sufficient regrowth to regenerate the cutblock.

\section{PLANT COMPOSITION CANOPY COVER(\%)}

\section{UNDERSTORY TREES}

BALSAM POPLAR

(Populus balsamifera) $\quad$ T $\quad 0-1 \quad 25$

ASPEN

(Populus tremuloides) $\quad 19 \quad 11-28 \quad 100$

SHRUBS

PRICKLY ROSE

$\begin{array}{llll}\text { (Rosa acicularis) } & 18 & 9-22 & 100\end{array}$

LOW BUSH CRANBERRY

(Viburnum edule) 2
SNOWBERRY OR BUCKBRUSH

(Symphoricarpos

$\begin{array}{llll}\text { occidentalis) } & 3 & 0-11 & 75\end{array}$

WILD RED RASPBERRY

(Rubus idaeus)

$5 \quad 0-16 \quad 50$

FORBS

WILD STRAWBERRY

(Fragaria virginiana) 22

PALMATE-LEAVED COLTSFoOT

(Petasites palmatus) $2 \quad 0-6 \quad 25$

DEWBERRY OR RUNNING RASPBERRY

$\begin{array}{llll}\text { (Rubus pubescens) } & 2 & 0-8 & 50\end{array}$

NORTHERN BEDSTRAW

(Galium boreale)

LINDLEY'S ASTER

$4 \quad 0-14 \quad 75$

(Aster ciliolatus)

$4 \quad 0-12 \quad 75$

GRASSES

MARSH REED GRASS

(Calamagrostis canadensis) 17

\section{ENVIRONMENTAL VARIABLES}

MOISTURE REGIME: MESIC

NUTRIENT REGIME: MESOTROPHIC

ELEVATION: 455-727(636) M

PERCENT SLOPE GRADIENT: LEVEL

SOIL DRAINAGE: WELL

RANGELAND HEALTH RATING: HEALTHY

\section{FORAGE PRODUCTION(KG/HA)}

$\begin{array}{ll}\text { GRASS } & 623 \\ \text { FORBS } & 580 \\ \text { SHRUBS } & 810 \\ \text { TOTAL } & 2013\end{array}$

\section{ECOLOGICALLY SUSTAINABLE STOCKING RATE $2.0 \mathrm{HA} / \mathrm{AUM}(0.2 \mathrm{AUM} / \mathrm{AC})$}




\section{DMC11. Pb/Honeysuckle (Populus balsamifera/Lonicera involcrata)}

$\mathbf{n}=\mathbf{8}$ This community type occupies mid to lower slope positions which receive nutrient rich seepage from upslope. It has similar moisture and nutrient regimes to the red osier dogwood dominated sites, but it has a very low cover of red osier dogwood which distinguishes this community type from the red osier dogwood dominated communities. The lack of red osier dogwood cover may be indicative of increased grazing pressure or this community may represent the transition to the Lower Foothills subregion. Indeed Lane et al. (2000) described an Aw/Honeysuckle in the Lower Foothills subregion and red osier dogwood was not as common in this subregion. This community type has a very diverse shrub and forb layer, but the high cover of shrubs often restricts access to livestock, limiting forage availability. This community type should be rated as secondary range.

\section{Plant COMPOSITION CANOPY COVER(\%)

MEAN RANGE CONST.

TREES

BALSAM POPLAR

(Populus balsamifera) $\quad 53 \quad 20-70 \quad 100$

ASPEN

(Populus tremuloides) $\quad 8 \quad 0-20 \quad 75$

SHRUBS

LOW BUSH CRANBERRY

(Viburnum edule) $\quad 4 \quad 0-20 \quad 63$

PRICKLY ROSE

(Rosa acicularis)

BRACTED HONEYSUCKLE

(Lonicera involucrata)

RASPBERRY

(Rubus idaeus)

FORBS

DEWBERRY OR RUNNING RASPBERRY

(Rubus pubescens) $\quad 3 \quad 0-10 \quad 88$

BUNCHBERRY

(Cornus canadensis) $\quad 4 \quad 0-20 \quad 88$

WILD STRAWBERRY

$\begin{array}{llll}\text { (Fragaria virginiana) } & 3 & 0-10 & 88\end{array}$

SHOWY ASTER

$\begin{array}{llll}\text { (Aster conspicuus) } & 3 & 1-10 & 100\end{array}$

TALL LUNGWORT

$\begin{array}{llll}\text { (Mertensia paniculata) } & 2 & 0-5 & 88\end{array}$

YELLOW PEAVINE

(Lathyrus ochroleucus) $110-3 \quad 88$

GRASSES

MARSH REED GRASS

(Calamagrostis canadensis)2 $\quad 1-3 \quad 100$

\section{ENVIRONMENTAL VARIABLES}

MOISTURE REGIME:

SUBHYGRIC

NUTRIENT REGIME:

RICH

ELEVATION:

590--648(630) M

PERCENT SLOPE GRADIENT:

0

SOIL DRAINAGE:

MODERATELY WELL

RANGELAND HEALTH RATING:

HEALTHY

FORAGE PRODUCTION(KG/HA)

GRASS $\quad 324$

FORBS $\quad 687$

SHRUBS $\quad 200$

TOTAL $1211 *$ ESTIMATE

ECOLOGICALLY SUSTAINABLE STOCKING

RATE

$1.5 \mathrm{HA} / \mathrm{AUM}(0.3 \mathrm{AUM} / \mathrm{AC})$ 


\section{DMC12. Pb/River alder \\ (Populus balsamifera/Alnus tenuifolia)}

$\mathbf{n}=\mathbf{2} \quad$ This community is found on lower slopes along natural drainages or areas with high water tables. River alder persists on moist sites and is replaced by green alder on drier upper slope positions. This community is similar to the $\mathrm{Pb}-\mathrm{Aw}$ /River alder community described by Beckingham and Archibald (1996) in the Boreal Mixedwood of Northern Alberta and is part of the dogwood ecosite. Production of this community type is very high because of the high moisture and nutrient conditions, however a large component of the total forage production is coming from alder which is generally unpalatable to livestock. This community should be rated as secondary or non-use range.

\section{Plant COMPOSITION CANOPY COVER(\%)} MEAN RANGE CONST.

\section{TREES}

BALSAM POPLAR

(Populus balsamifera) $\quad 50 \quad 50-60 \quad 100$

SHRUBS

PRICKLY ROSE

(Rosa acicularis)

RIVER ALDER

$5 \quad 1-10 \quad 100$

(Alnus tenuifolia)

RED OSIER DOGWOOD

(Cornus stolonifera)

FORBS

HORSETAIL

(Equisetum arvense) $\quad 5 \quad 1-10 \quad 100$

DANDELION

(Taraxacum officinale)

STAR FLOWERED SOLOMON SEAL

(Smilacina stellata)

$55 \quad 50-60 \quad 100$

$12 \quad 3-20 \quad 100$

$1-3+100$

$1-3 \quad 100$

\section{GRASSES}

MARSH REED GRASS

(Calamagrostis canadensis)1

HAIRY WILDRYE

(Elymus innovatus)
$1-2 \quad 100$

$1 \quad 1-2 \quad 100$

\section{$0-1 \quad 50$}

$0-1 \quad 50$

\section{ENVIRONMENTAL VARIABLES}

MOISTURE REGIME:

SUBHYGRIC

NUTRIENT REGIME:

$\mathrm{RICH}$

ELEVATION:

$556-646(587) M$

PERCENT SLOPE GRADIENT:

$1-5(3) \%$

SOIL DRAINAGE:

MODERATELY WELL

RANGELAND HEALTH RATING:

HEALTHY

\section{FORAGE PRODUCTION(KG/HA)}

$\begin{array}{ll}\text { GRASS } & 245 \\ \text { FORBS } & 544 \\ \text { SHRUBS } & 397 \\ \text { TOTAL } & 1187 \text { *ESTIMATE }\end{array}$

\section{ECOLOGICALLY SUSTAINABLE STOCKING RATE \\ NON-USE}

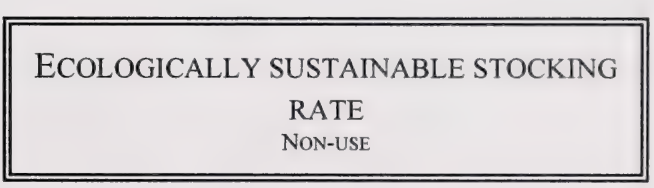




\section{DMC13. Pb-Aw/Silverberry \\ (Populus balsamifera-P. tremuloides/Elaeagnus commutata)}

$\mathbf{n}=3$ This community is scattered throughout the Dry Mixedwood subregion. It appears to represent the invasion of aspen and balsam poplar onto silverberry shrublands. Silverberry thickets can occur on alluvial floodplain terraces, in V-shaped ravines and swale-like depressions where overland flows provide additional moisture (Thompson and Hansen 2002). The open nature and high productivity on these silverberry dominated communities make them attractive to livestock grazing. The understory of these communities are often dominated by Kentucky bluegrass, smooth brome and dandelion. Thompson and Hansen (2002) felt that silverberry dominated communities represented a grazing disclimax of red osier dogwood communities in Southern Alberta. This community should be rated as secondary range for domestic livestock.

\section{PLANT COMPOSITION CANOPY COVER(\%) MEAN RANGE CONST.}

TREES

TREMBLING ASPEN

(Populus tremuloides)

$17 \quad 0-50 \quad 33$

BALSAM POPLAR

(Populus balsamifera)

SHRUBS

PRICKLY ROSE

(Rosa acicularis)

YELLOW WILLOW

(Salix lutea)

SILVERBERRY

(Elaeagnus commutata)

FORBS

DANDELION

(Taraxacum officinale)

SHOWY ASTER

(Aster conspicuus)

HORSETAIL

STRAWBERRY

(Fragaria virginiana)

YELLOW PEAVINE

(Lathyrus ochroleucus)

GRASSES

KENTUCKY BLUEGRASS

(Poa pratensis)

SMOOTH BROME

(Bromus inermis)

$31 \quad 3-50 \quad 100$

$11 \quad 3-20 \quad 100$

$4 \quad 1-10 \quad 100$

$23 \quad 20-30 \quad 100$

$2 \quad 1-3 \quad 100$

$1 \quad 1-3 \quad 100$

$4 \quad 0-10 \quad 66$

$4 \quad 0-10 \quad 66$

$1 \quad 1-2 \quad 100$

$5 \quad 1-10 \quad 100$

$4 \quad 0-10 \quad 66$
ENVIRONMENTAL VARIABLES

MOISTURE REGIME: SUBHYGRIC

NUTRIENT REGIME: $\mathrm{RICH}$

ELEVATION: 570-690(630) M

SOIL DRAINAGE: MODERATELY WELL

RANGELAND HEALTH RATING: HEALTHY

\section{FORAGE PRODUCTION(KG/HA)}

$\begin{array}{ll}\text { GRASS } & 200 \\ \text { FORBS } & 300 \\ \text { SHRUBS } & 600 \\ \text { TOTAL } & 1100 \text { *ESTIMATE }\end{array}$

\footnotetext{
ECOLOGICALLY SUSTAINABLE STOCKING RATE

$1.5 \mathrm{HA} / \mathrm{AUM}(0.3 \mathrm{AUM} / \mathrm{AC})$
} 


\section{DMC14. Pb/Snowberry \\ (Populus balsamifera/Symphoricarpos occidentalis)}

$\mathbf{n}=\mathbf{3}$ This community type occupies small seepage areas on slopes above creeks and rivers in the Dry Mixedwood subregion. Snowberry prefers well drained habitats and has been found to quite common on forested slopes and river flood plains throughout the Boreal forest (Lane et al. 2000). The presence of balsam poplar indicates that the moisture content is sufficient to support its growth in this community. This community type is usually found in only small isolated spots. Consequently it will contribute little to the overall carrying capacity of a lease.

\section{Plant COMPOSITION CANOPY COVER(\%)}

MEAN RANGE CONST.

\section{TREES}

TREMBLING ASPEN

(Populus tremuloides)

BALSAM POPLAR

(Populus balsamifera)

SHRUBS

PRICKLY ROSE

(Rosa acicularis)

SNOWBERRY

(Symphoricarpos

occidentalis)
RED OSIER DOGWOOD

(Cornus stolonifera)

FORBS

BUNCHBERRY

(Cornus canadensis)

STRAWBERRY

(Fragaria virginiana)

NORTHERN BEDSTRAW

(Galium boreale)

DANDELION

(Taraxacum officinale)

YELLOW PEAVINE

(Lathyrus ochroleucus)

GRASSES

MARSH REED GRASS

(Calamagrostis canadensis)1

KENTUCKY BLUEGRASS

(Poa pratensis)

$5 \quad 0-11 \quad 66$

$36 \quad 3-80 \quad 100$

$17 \quad 10-20 \quad 100$

$22 \quad 6-20 \quad 100$

$5 \quad 1-10 \quad 100$

$3 \quad 0-6 \quad 66$

$2 \quad 1-3 \quad 100$

$1 \quad 1-2 \quad 100$

$2 \quad 0-3 \quad 66$

$6 \quad 3-15 \quad 100$

0-1 $1 \quad 66$

$3 \quad 0-10 \quad 33$

\section{ENVIRONMENTAL VARIABLES}

MOISTURE REGIME:

SUBHYGRIC

NUTRIENT REGIME:

$\mathrm{RICH}$

ELEVATION:

556-709(624) M

SOIL DRAINAGE:

MODERATELY WELL

RANGELAND HEALTH RATING:

HEALTHY

\section{FORAGE PRODUCTION(KG/HA)}

GRASS $\quad 18$

FORBS $\quad 230$

SHRUBS $\quad 896$

TOTAL $1204 *$ EsTIMATE

ECOLOGICALLY SUSTAINABLE STOCKING

RATE

1.5 HA/AUM (0.3 AUM/AC) 


\section{DMC15. Pb/Reedgrass \\ (Populus balsamifera/Calamagrostis stricta) \\ (Populus balsamifera/Calamagrostis stricta)}

$\mathbf{n}=\mathbf{2}$ This community type is not common in the Dry Mixedwood subregion. It appears to represent the invasion of balsam poplar onto reedgrass and reed canary grass dominated meadows. As sloughs and small lakes dry up the edge communities become drier which favours the growth of trees and shrubs. If drying continues this community will likely succeed to a $\mathrm{Pb} / \mathrm{Red}$ osier dogwood dominated community type. However, if flooding increases balsam poplar will likely decline. This community type is very productive for domestic livestock and the open nature of the understory also allows for good access. This community should be rated as primary range.

\section{PLANT COMPOSITION CANOPY COVER(\%)}

\section{TREES}

BALSAM POPLAR

(Populus balsamifera) $\quad 40 \quad 20-60 \quad 100$

SHRUBS

RED OSIER DOGWOOD

(Cornus stolonifera)

\section{FORBS}

THISTLE

(Cirsium arvense)

HORSETAII

(Equisetum arvense)

SOW THISTLE

(Sonchus arvensis)

DANDELION

(Taraxacum officinale)

GRASSES

NARROW REED GRASS

(Calamagrostis stricta)

REED CANARY GRASS

(Phalaris arundinacea)

WATER SEDGE

(Carex aquatilis)

\section{MEAN RANGE CONST.}




\section{DMC16. Bw/Labrador tea \\ (Betula papyrifera/Ledum groenlandicum)}

$\mathbf{n = 1} \quad$ This community type represents a treed poor fen ecosite that was recently burned in Elk Island National Park. The poor fen ecosite is intermediate in nutrient regime between the bog and the rich fen ecosites (Beckingham and Archibald 1996). The presence of Labrador tea and short sedge is indicative of the acidic soil conditions. Treed poor fens are often dominated by black spruce in the Boreal Mixedwood (Beckingham and Archibald 1996), however the frequent fire regime in the park has burned the black spruce canopy and the site has become dominated by paper birch a early successional species in these boggy areas. These boggy community types are often too wet for domestic livestock and the species growing in them are often unpalatable. This community should be rated as non-use.

\section{Plant COMPOSITION Canopy Cover(\%)}

\section{TREES}

MEAN RANGE CONST.

PAPER BIRCH

(Betula papyrifera)

ASPEN

$\begin{array}{llll}\text { (Populus tremuloides) } & 1 & - & 100\end{array}$

SHRUBS

LABRADOR TEA

(Ledum groenlandicum) $40 \quad-\quad 100$

CURRANT

(Ribes triste)

RASPBERRY

(Rubus idaeus)

BLUEBERRY

(Vaccinium myrtilloides) $20 \quad-\quad 100$

FORBS

FIREWEED

(Epilobium angustifolium) $1 \quad-\quad 100$

SKULL CAP

(Scutellaria galericulata) $1 \quad$ - $\quad 100$

GRASSES

MARSH REED GRASS

(Calamagrostis canadensis)3 - 100

SHORT SEDGE

(Carex curta)

100

\section{ENVIRONMENTAL VARIABLES}

MOISTURE REGIME:

SUBHYDRIC

NUTRIENT REGIME:

POOR

ELEVATION:

$625 \mathrm{M}$

SOIL DRAINAGE:

POORLY

RANGELAND HEALTH RATING:

HEALTHY

FORAGE PRODUCTION(KG/HA)

GRASS $\quad 150$

FORBS $\quad 250$

SHRUBS $\quad 350$

TOTAL $\quad 750 *$ ESTIMATE
ECOLOGICALLY SUSTAINABLE STOCKING

RATE

NON-USE 


\section{DMC17. Bw/Raspberry \\ (Betula papyrifera/Rubus idaeus)}

$\mathbf{n}=2 \quad$ This community type was described adjacent to old beaver dams. Cutting of the adjacent tree canopy and the increased moisture around the dam favours the growth of paper birch and raspberry. Both species are early successional and will rapidly dominate a site after disturbance. As the site drys and undergoes succession it will likely succeed to willow and eventually balsam poplar and white spruce. This community occupies small areas adjacent to the ponds and sloughs and is generally too wet for livestock. It should be rated as non-use.

\section{PLANT COMPOSITION CANOPY COVER(\%)} MEAN RANGE CONST.

\section{TREES}

PAPER BIRCH

$\begin{array}{llll}\text { (Betula papyrifera) } & 85 & 80-90 & 100\end{array}$

ASPEN

(Populus tremuloides) $\quad 1 \quad 0-1 \quad 50$

SHRUBS

ROSE

\section{(Rosa acicularis)}

SCOULER'S WILLOW

(Salix scouleriana)

RASPBERRY

(Rubus ideaus)

FORBS

FIREWEED

(Epilobium angustifolium) 1

BUNCHBERRY

(Cornus cornuta) $2 \quad 0-3 \quad 50$

GRASSES

MARSH REED GRASS

(Calamagrostis canadensis) 15

\section{ENVIRONMENTAL VARIABLES}

\author{
MOISTURE REGIME: \\ SUBHYDRIC \\ NUTRIENT REGIME: \\ $\mathrm{RICH}$ \\ ELEVATION: \\ $625 \mathrm{M}$ \\ SOIL DRAINAGE: \\ POORLY \\ RANGELAND HEALTH RATING: \\ HEALTHY
}

\section{FORAGE PRODUCTION(KG/HA)}

$\begin{array}{ll}\text { GRASS } & 300 \\ \text { FORBS } & 300 \\ \text { SHRUBS } & 400 \\ \text { TOTAL } & 1000 \text { *ESTIMATE }\end{array}$

ECOLOGICALLY SUSTAINABLE STOCKING

RATE

NON-USE 


\section{DMC18. Pb-Bw/Kentucky bluegrass \\ (Populus balsamifera-Betula papyrifera/Poa pratensis)}

$\mathbf{n}=\mathbf{5}$ This community represents a $\mathrm{Pb}$ or $\mathrm{Bw} / \mathrm{Red}$ osier dogwood community that has recieved prolonged heavy grazing. This community type often occurs in relatively small isolated patches created by intensive grazing adjacent to water, salt or temporary holding areas. The species richness and diversity of native shrubs, forbs, and grass is reduced and replaced by grazing resistant species like clover, dandelion and Kentucky bluegrass.

\section{PLANT COMPOSITION CANOPY COVER(\%)}

TREES

TREMBLING ASPEN

(Populus tremuloides)

BALSAM POPLAR

(Populus balsamifera) $\quad 54 \quad 30-80 \quad 100$

PAPER BIRCH

$\begin{array}{llll}\text { (Betula papyrifera) } & 8 & 0-40 & 60\end{array}$

SHRUBS

WILLOW SPP.

(Salix spp.)

WILD RED RASPBERRY

(Rubus idaeus)

SNOWBERRY

(Symphoricarpos

occidentalis)

PRICKLY ROSE

(Rosa acicularis)

FORBS

MEAN RANGE CONST.

FIREWEED

(Epilobium angustifolium) 1

$3 \quad 3-4 \quad 100$

$4 \quad 0-10 \quad 80$

DEWBERRY OR RUNNING RASPBERRY

(Rubus pubescens) $\quad 4 \quad 0-20 \quad 80$

ClOVER

(Trifolium spp.)

$2 \quad 0-10 \quad 60$

WILD STRAWBERRY

$\begin{array}{llll}\text { (Fragaria virginiana) } & 3 & 1-3 & 100\end{array}$

SHOWY ASTER

$\begin{array}{llll}\text { (Aster conspicuus) } & 2 & 1-3 & 100\end{array}$

Horsetail

$\begin{array}{llll}\text { (Equisetum arvense) } & 2 & 1-3 & 100\end{array}$

DANDELION

(Taraxacum officinale) $4 \quad 1-10 \quad 100$

GRASSES

MARSH REED GRASS

(Calamagrostis canadensis)1
KENTUCKY BLUEGRASS

$\begin{array}{llll}\text { (Poa pratensis) } & 9 & 1-20 & 100 \\ \text { QUACKGRASS } & & & \\ \text { (Agropyron repens) } & 1 & 0-3 & 60\end{array}$

(Agropyron repens) $\quad 1 \quad 0-3 \quad 60$

\section{ENVIRONMENTAL VARIABLES}

MOISTURE REGIME:

SUBHYGRIC

NUTRIENT REGIME: $\mathrm{RICH}$

ELEVATION: 455-697(524) M

PERCENT SLOPE GRADIENT:

$0-5$

SOIL DRAINAGE:

MODERATELY WELL

RANGELAND HEALTH RATING:

UNHEALTHY

\section{FORAGE PRODUCTION(KG/HA)}

$\begin{array}{ll}\text { GRASS } & 700 \\ \text { FORBS } & 300 \\ \text { SHRUBS } & 150 \\ \text { TOTAL } & 1150 * \text { ESTIMATE }\end{array}$

\section{ECOLOGICALLY SUSTAINABLE STOCKING RATE \\ $2.0 \mathrm{HA} / \mathrm{AUM}(0.2 \mathrm{AUM} / \mathrm{AC})$}




\section{DMC19. Pb/Smooth brome \\ (Populus balsamifera/Bromus inermis)}

$\mathbf{n}=\mathbf{2}$ This community type is similar to the previously described red osier dogwood dominated balsam poplar dominated community types, but has a high cover of smooth brome in the understory. Smooth brome is an introduced grass that can increase with increased grazing pressure, but smooth brome is also highly invasive and can invade into ungrazed areas. The invasion of non-native invaders onto the site makes this community moderately productive for domestic livestock. .

\section{Plant COMPOSITION CANOPY COVER(\%)}

\section{TREES}

BALSAM POPLAR

$\begin{array}{llll}\text { (Populus balsamifera) } \quad 70 & 60-80 & 100\end{array}$

SHRUBS

WILLOW SPP.

(Salix spp.)

WILD RED RASPBERRY

$\begin{array}{llll}\text { (Rubus idaeus) } & 10 & 0-20 & 50\end{array}$

SNOWBERRY

(Symphoricarpos

$\begin{array}{llll}\text { occidentalis) } & 7 & 3-10 & 100\end{array}$

RED OSIER DOGWOOD

(Cornus stolonifera) $\quad 10 \quad 1-20 \quad 100$

FORBS

Clover

$\begin{array}{llll}\text { (Trifolium spp.) } & 1 & 0-1 & 50\end{array}$

HORSETAIL

$\begin{array}{llll}\text { (Equisetum arvense) } & 5 & 1-10 & 100\end{array}$

DANDELION

$\begin{array}{llll}\text { (Taraxacum officinale) } \quad 2 & 1-3 & 100\end{array}$

STAR FLOWERED SOLOMON SEAL

$\begin{array}{llll}\text { (Smilacina stellata) } & 7 & 3-10 & 100\end{array}$

SHOWY ASTER

$\begin{array}{llll}\text { (Aster conspicuus) } & 2 & 1-3 & 100\end{array}$

RICHARDSON GERANIUM

(Geranium richardsonii) $\quad 10 \quad 0-20 \quad 50$

HEMP-NETTLE

(Galeopsis tetrahit) $\quad 5 \quad 0-10 \quad 50$

GRASSES

SMOOTH BROME

$\begin{array}{llll}\text { (Bromus inermis) } & 10 & 1-20 & 100\end{array}$

KENTUCKY BLUEGRASS

(Poa pratensis)

(Agropyron repens)

$\begin{array}{lll}10 & 1-20 & 100\end{array}$

$5 \quad 0-10 \quad 50$
$2 \quad 0-3 \quad 50$

\section{ENVIRONMENTAL VARIABLES}

MOISTURE REGIME:

SUBHYGRIC

NUTRIENT REGIME:

RICH

ELEVATION:

455-697(524) M

PERCENT SLOPE GRADIENT:

0 - 5

SOIL DRAINAGE:

WELL TO MODERATELY WELL

RANGELAND HEALTH RATING:

UNHEALTHY

\section{FORAGE PRODUCTION(KG/HA)}

$\begin{array}{ll}\text { GRASS } & 900 \\ \text { FORBS } & 300 \\ \text { SHRUBS } & 50 \\ \text { TOTAL } & 1250 * \text { ESTIMATE }\end{array}$

\begin{tabular}{|}
\hline ECOLOGICALLY SUSTAINABLE STOCKING \\
RATE \\
$2.0 \mathrm{HA} / \mathrm{AUM}(0.2 \mathrm{AUM} / \mathrm{AC})$ \\
\hline
\end{tabular}




\section{DRY MIXEDWOOD SUBREGION}

\section{CONIFEROUS AND MIXEDWOOD FOREST COMMUNITIES}

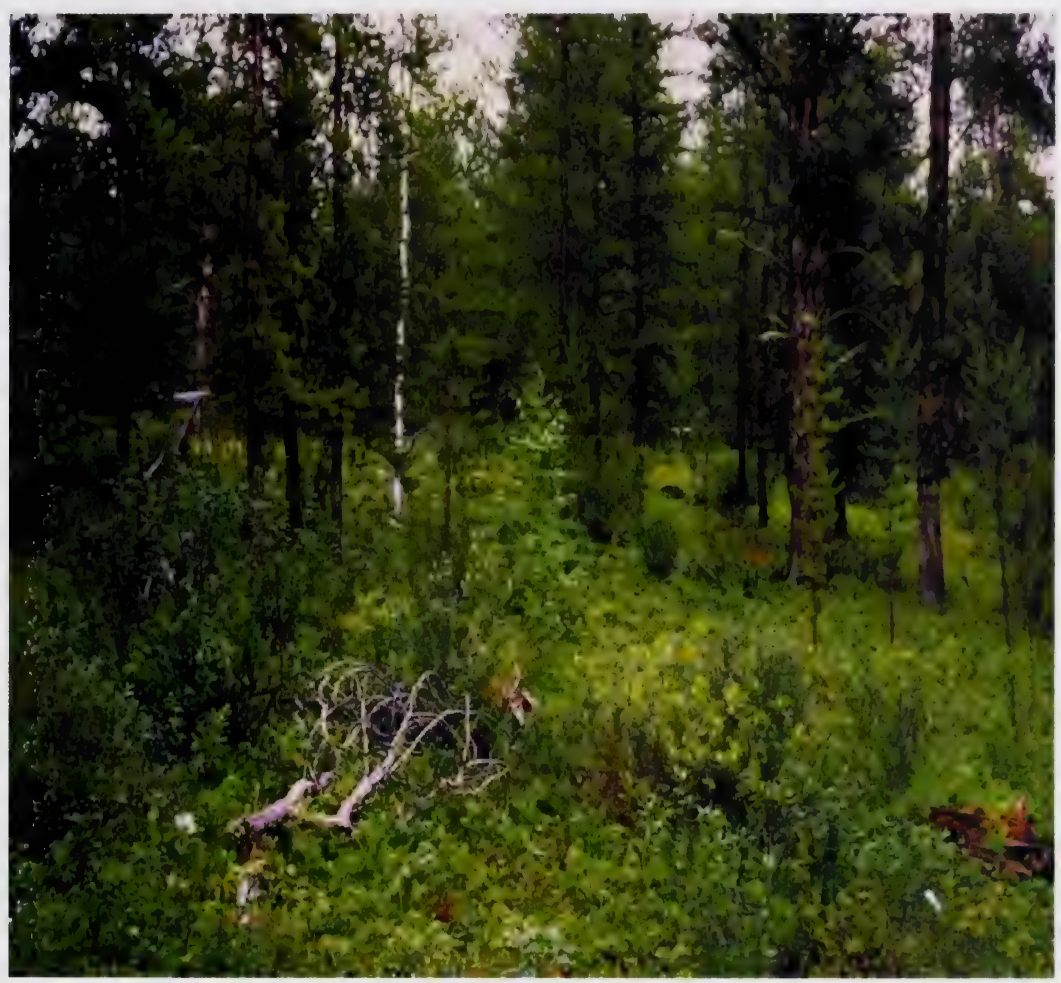

Photo 6. $\mathrm{Pj} /$ Bearberry community type in the Dry Mixedwood subregion 


\section{DM-CONIFEROUS AND MIXEDWOOD FORESTS}

The mixedwood and coniferous community types described in this guide represent seven ecological sites (ecosites) as described by Beckingham and Archibald (1996). On sites with subxeric moisture and poor nutrient regimes, coarse textured, sandy soils open stands of jack pine generally dominate $(\mathrm{Pj} / \mathrm{Alder}, \mathrm{Pj} / \mathrm{Bearberry})$. These community types commonly have a carpet of lichens covering the forest floor and a thin organic layer typically less than $5 \mathrm{~cm}$ thick (Beckingham and Archibald 1996).

On slightly moister sites with submesic moisture and medium nutrient regimes aspen grows in conjunction with jack pine to form the $\mathrm{Pj}-\mathrm{Aw} / \mathrm{Bearberry}$ community type. On slightly moister sites Aw-Sw/Bearberry and Sw/Buffaloberry/Bearberry communities are found. The soils of these community types continue to be coarse-textured but the moisture and nutrient conditions are more favourable to the growth of aspen and spruce.

The mesic/medium sites are generally dominated by white spruce (Sw/Moss) and mixedwood communities of aspen and spruce (Aw-Sw/Rose/Marsh reedgrass, $\mathrm{Aw}-\mathrm{Pb}$ $\mathrm{Sw} / \mathrm{Willow} / \mathrm{Wild}$ sarsparilla, Sw-Pb-Aw/Rose/Twinflower, Sw-Aw/Low bush cranberry). These communities represent the reference ecological site for the Boreal Mixedwood subregion (Beckingham and Archibald 1996). Generally, these sites have moderately fine to fine-textured till or glaciolacustrine parent materials. Pioneer deciduous species (aspen, balsam poplar and birch) are replaced with white spruce and balsam fir as these sites develop successionally. With succession shade tolerant plants take over the herbaceous layer as conifers dominate the canopy. These shade tolerant species are unproductive and often unpalatable for domestic livestock. Forage productivity declines from $2.3 \mathrm{ha} / \mathrm{AUM}$ in a deciduous community to $2.3-8.6 \mathrm{ha} / \mathrm{AUM}$ in a mixedwood community to less than 10 ha/AUM in a conifer community.

Black spruce and larch communites generally dominate on wetter sites with subhygric to subhydric moisture regimes and poor to medium nutrient regimes to form the $\mathrm{Sb} / \mathrm{Willow} / \mathrm{Moss}$ and $\mathrm{Sb}-\mathrm{Lt} / \mathrm{Labrador}$ tea/Moss community types. Larch is more tolerant of excessive moisture and is indicative of an enriched nutrient status, while black spruce is typical in areas of stagnating ground water with poor nutrient status (Hay et al. 1985). Generally, these community types are considered non-use for domestic livestock. In contrast on the richer sites red osier dogwood and horsetail dominate the understory to form the Sw/Horsetail and $\mathrm{Sw}-\mathrm{Pb} / \mathrm{Red}$ osier dogwood dominated communities.

Beckingham and Archibald (1996), provide a good description on how the conifer and mixedwood community types are arranged in the landscape. 


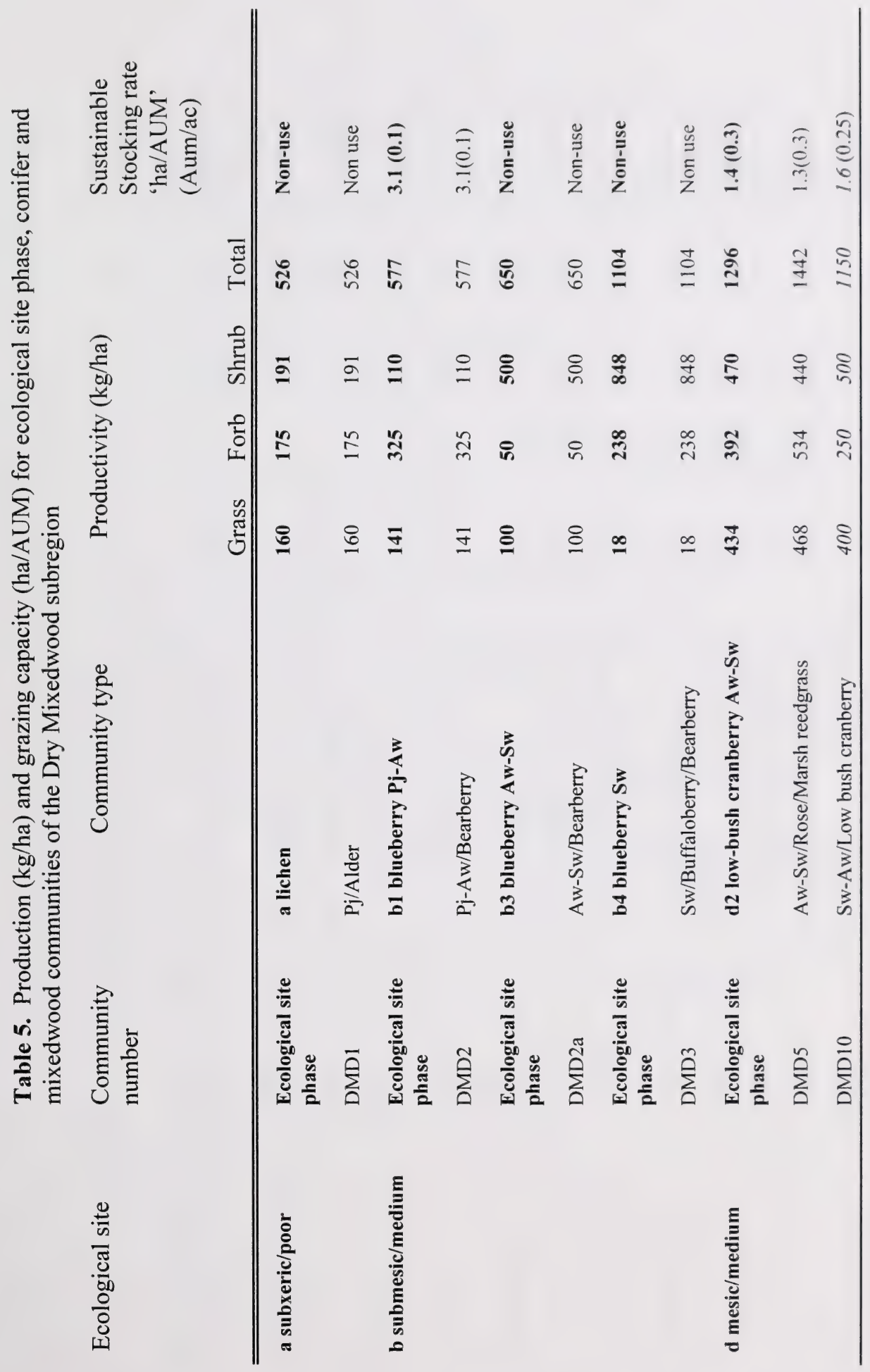




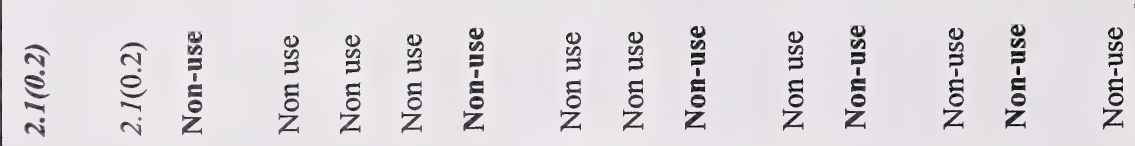

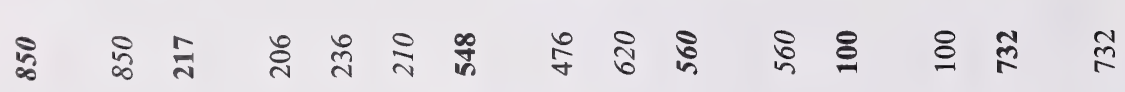

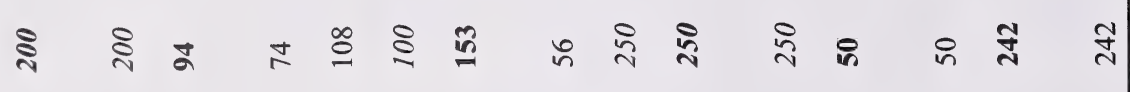

ㅇํㅇ

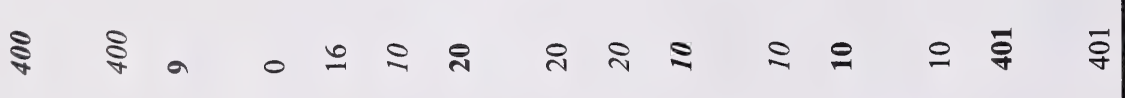

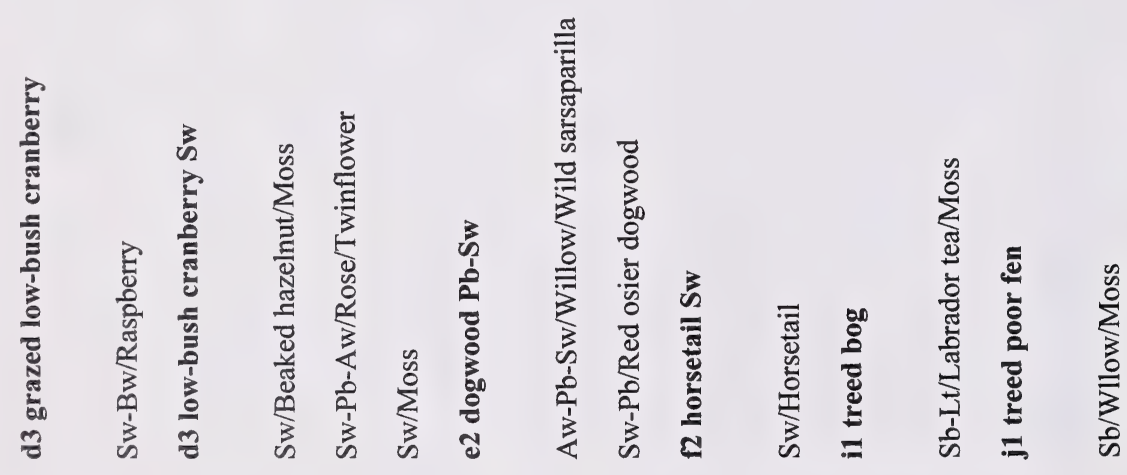

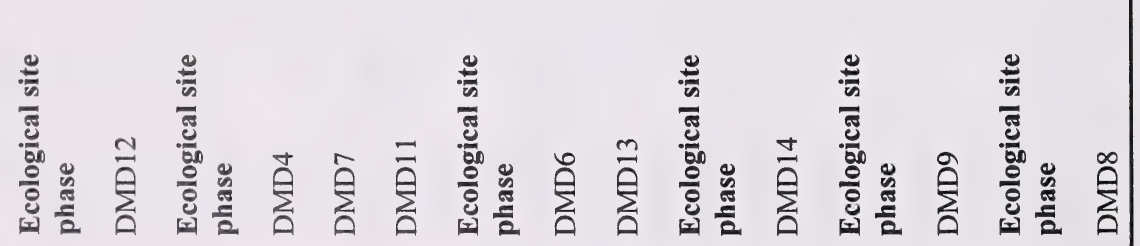

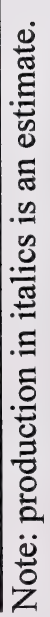




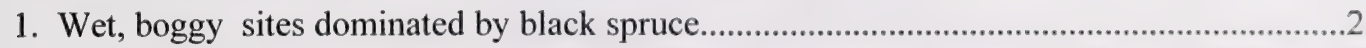
Moist, mesic or dry sandy sites dominated by spruce, aspen, balsam poplar or jackpine... .3

2. Richer nutrient sites with willow and sedge dominating understory Sb/Willow/Moss (DMD8) Poorer sites with Labrador tea and larch present..........Sb-Lt/Labrador Tea/Moss (DMD9)

3. Mesic or subhygric sites dominated by spruce, aspen, balsam poplar.................................

Dry, sandy sites dominated by jack pine............................................................................

4. Mixedwood types dominated by a mixture of conifer and deciduous trees.........................

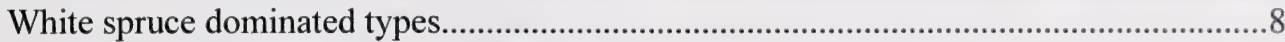

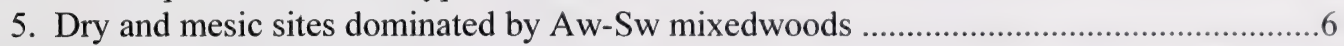

Balsam poplar present, moister, richer sites....................................................................

6. Typical mesic site, with rose and marsh reedgrass. Aw-Sw/Rose/Marsh Reedgrass (DMD5)

Drier sites dominated by bearberry Aw-Sw/Bearberry (DMD2a)

7. Willow, wild sarsaparilla, red osier dogwood or horsetail dominate understory.... Twinflower dominates understory, poorer nutrient sites .Sw-Pb-Aw/Rose/Twinflower (DMD7)

8. Mesic sites dominated by hazelnut, moss, low bush cranberry or raspberry.....

Poorer nutrient sites, buffaloberry, bearberry dominate understory. Sw/Buffaloberry/Bearberry (DM

9. Bearberry dominates, alder low in cover or absent. Alder dominates understory. .Pj-Aw/Bearberry (DMD2)

10. Hazelnut dominates the understory. Pj/Alder (DMD1) Mesic sites dominated by low bush cranberry, moss or raspberry..................................11

11. Moss dominates understory, little shrub cover. Sw/Moss (DMD11) Raspberry or Low bush cranberry dominate the understory...

12. Raspberry dominates understory, disturbed sites......Sw-Bw/Raspberry (DMD12) Low bush cranberry dominates understory...........Sw-Aw/Low bush cranberry (DMD10)

13. Willow dominated understory. Sw-Pb-Aw/Willow/Moss (DMD6) Red osier dogwood or horsetail dominates understory

14. Red osier dogwood dominates understory Horsetail dominates understory. $\mathrm{Sw}-\mathrm{Pb} / \mathrm{Red}$ osier dogwood (DMD13) Sw/Horsetail (DMD14) 


\section{DMD1. Pj/Alder \\ (Pinus banksianal Alnus crispa)}

$\mathbf{n}=2$ This community type is found on dry, rapidly drained, sandy soils with a poor nutrient status. Consequently, production is quite low. Cattle will utilize these areas due to the easy access, however overutilization will quickly deplete the area of forage. This community type would be rated as secondary or non-use range.

\section{PERCENT COMPOSITION CANOPY COVER(\%)} MEAN RANGE CONST.

TREES

JACK PINE

(Pinus banksiana)

$43 \quad 35-50 \quad 100$

SHRUBS

GREEN ALDER

(Alnus crispa)

PRICKLY ROSE

(Rosa acicularis)

SASKATOON

$\begin{array}{llll}\text { (Amelanchier alnifolia) } & 5 & 1-8 & 100\end{array}$

FORBS

TWIN-FLOWER

(Linnaea borealis)

BEARBERRY

(Arctostaphylos uva-ursi) $9 \quad 0-18 \quad 50$

YELLOW PEAVINE

(Lathyrus ochroleucus) $\quad 4 \quad 0-8 \quad 50$

STRAWBERRY

Fragaria virginiana) $\quad 2 \quad 1-2 \quad 100$

GRASSES

SEDGES

(Carex spp.)

HAIRY WILD RYE

(Elymus innovatus)

NORTHERN RICEGRASS

(Oryzopsis pungens)
$6 \quad 0-12 \quad 50$

$33 \quad 30-35 \quad 100$

$9 \quad 7-10 \quad 100$

100

\section{0}

50

100

$1-11 \quad 100$

$3 \quad 1-4 \quad 100$

$6 \quad 1-10 \quad 100$
ENVIRONMENTAL VARIABLES

MOISTURE REGIME:

SUBXERIC

NUTRIENT REGIME

POOR

ELEVATION:

$606 \mathrm{M}$

SOIL DRAINAGE:

RAPIDLY

PERCENT SLOPE GRADIENT:

$2-8 \%$

RANGELAND HEALTH RATING:

HEALTHY

FORAGE PRODUCTION(KG/HA)

$\begin{array}{ll}\text { GRASS } & 160 \\ \text { FORBS } & 175 \\ \text { SHRUBS } & 191\end{array}$

ECOLOGICALLY SUSTAINABLE STOCKING RATE NON-USE 


\section{DMD2. Pj-Aw/Bearberry \\ (Pinus banksiana/Arctostaphylos uva-ursi)}

$\mathbf{n = 4}$ This community represents a jack pine forest with a secondary canopy of aspen. It is very similar to the Pj/Alder community type, but it is found on slightly moister soils with better nutrient regimes. These conditions favour the growth of aspen. Like the previous community, cattle will utilize these areas due to the easy access, however over-utilization will quickly deplete the forage supply. This community type would be rated as secondary range and should be grazed on a single rotation per year.

Plant Composition Canopy Cover(\%)

\section{TREES}

MEAN RANGE CONST.

JACK PINE

(Pinus banksiana) $\quad 45 \quad 30-45 \quad 100$

ASPEN

(Populus tremuloides) $\quad 13 \quad 10-20 \quad 100$

SHRUBS

SASKATOON

(Amelanchier alnifolia) $\quad 9 \quad 1-15 \quad 100$

PRICKLY ROSE

$\begin{array}{llll}\text { (Rosa acicularis) } & 6 & 4-8 & 100\end{array}$

BLUEBERRY

(Vaccinium myrtilloides) $7 \quad 0-2 \quad 75$

FORBS

BEARBERRY

(Arctostaphylos uva-ursi) $15 \quad 7-64 \quad 100$

NORTHERN BEDSTRAW

(Galium boreale) $2 \quad 1-3 \quad 100$

WILD LILY-OF-THE-VALLEY

(Maianthemum canadense)2

CREAM-COLOURED VETCHLING

(Lathyrus ochroleucus) $\quad 7 \quad 3-7 \quad 100$

GRASSES

HAIRY WILD RYE

(Elymus innovatus)

MOSSES

$10 \quad 2-16 \quad 100$

Moss SPP.

2

$0-7 \quad 25$

\section{ENVIRONMENTAL VARIABLES}

MOISTURE REGIME:

SUBMESIC

NUTRIENT REGIME:

POOR

ELEVATION:

$606 \mathrm{M}$

SOIL DRAINAGE:

RAPIDLY

PERCENT SLOPE GRADIENT:

$0-5$

RANGELAND HEALTH RATING:

HEALTHY

\section{FORAGE PRODUCTION(KG/HA)}

$\begin{array}{ll}\text { GRASS } & 141 \\ \text { FORBS } & 325 \\ \text { SHRUBS } & 110 \\ \text { TOTAL } & 577\end{array}$

ECOLOGICALLY SUSTAINABLE STOCKING RATE $3.1 \mathrm{HA} / \mathrm{AUM}(<0.1 \mathrm{AUM} / \mathrm{AC})$ 


\section{DMD2a. Aw-Sw/Bearberry \\ (Populus tremuloides-Picea glauca/Arctostaphylos uva-ursi)}

$\mathbf{n}=\mathbf{1}$ This community type was found on a small, sandy hillcrest with a high water table. It is similar to the Sw/Buffaloberry/Bearberry (DMD3) community type, but this community is successionally younger. The majority of productivity is from bearberry which is unpalatable to livestock. Consequently, this community type would be considered nonuse range.

\section{Plant COMPOSITION CANOPY COVER(\%)} MEAN RANGE CONST.

\section{TREES}

WHITE SPRUCE

(Picea glauca)

ASPEN

(Populus tremuloides)

SHRUBS

SASKATOON

(Amelanchier alnifolia) $1 \quad-\quad 100$

PRICKLY ROSE

$\begin{array}{llll}\text { (Rosa acicularis) } & 2 & - & 100\end{array}$

BLUEBERRY

(Vaccinium myrtilloides) $2 \quad$ - 100

FORBS

BEARBERRY

(Arctostaphylos uva-ursi) $37 \quad-\quad 100$

STRAWBERRY

(Fragaria virginiana) $\quad 1 \quad-\quad 100$

YELLOW PEAVINE

$\begin{array}{lll}\text { (Lathyrus ochroleucus) } & 4 \quad-\quad 100\end{array}$

WILD-LILY-OF-THE VALLEY

(Maianthemum canadense)3

GRASSES

HAIRY WILDRYE

(Elymus innovatus)

NORTHERN RICEGRASS

(Oryzopsis pungens)

\section{ENVIRONMENTAL VARIABLES}

MOISTURE REGIME:

SUBMESIC

NUTRIENT REGIME:

POOR

ELEVATION:

$606 \mathrm{M}$

SOIL DRAINAGE:

WELL

PERCENT SLOPE GRADIENT:

$$
0-4
$$

RANGELAND HEALTH RATING:

HEALTHY

FORAGE PRODUCTION(KG/HA)

GRASS $\quad 100$

FORBS 50

SHRUBS $\quad 500$

TOTAL 650

ECOLOGICALLY SUSTAINABLE STOCKING RATE NON-USE 


\section{DMD3. Sw/Buffaloberry/Bearberry \\ (Picea glauca/ Shepherdia canadensis/Arctostaphylos uva-ursi)}

$\mathbf{n = 1}$ This community type represents a very open spruce forest. It was found on a small, sandy hillcrest with a high water table. The site may have a high $\mathrm{pH}$ and be somewhat nutrient poor as indicated by the abundance of buffaloberry (Beckingham 1993). The majority of productivity is from buffaloberry which is unpalatable to livestock. Consequently. this community type would be considered non-use range.

\section{Plant COMPOSITION CANOPY COVER(\%)} MEAN RANGE CONST.

\section{TREES}

WHITE SPRUCE

(Picea glauca)

$$
10
$$

100

SHRUBS

BUFFALOBERRY

(Shepherdia canadensis) 48

PRICKLY ROSE

(Rosa acicularis)

BLUEBERRY

(Vaccinium myrtilloides)

SNOWBERRY

(Symphoricarpos

occidentalis)

5

FORBS

BEARBERRY

(Arctostaphylos uva-ursi) 19

TWINFLOWER

(Linnaea borealis)

YELLOW PEAVINE

(Lathyrus ochroleucus)

TOADFLAX

(Comandra umbellata)

GRASSES

MOUNTAIN RICEGRASS

(Oryzopsis asperifolia)

NORTHERN RICEGRASS

(Oryzopsis pungens)

SEDGE

(Carex spp.)

100

100

100

100

100

100

100

$2 \quad-\quad 100$

$8 \quad-\quad 100$

$6 \quad-\quad 100$

$5 \quad-\quad 100$

\section{ENVIRONMENTAL VARIABLES}

MOISTURE REGIME:

SUBMESIC

NUTRIENT REGIME:

POOR
ECOLOGICALLY SUSTAINABLE STOCKING RATE NON-USE

\section{ELEVATION:}

$606 \mathrm{M}$

SoIl DRAINAGE:

WELL

PERCENT SLOPE GRADIENT:

0 - 4

RANGELAND HEALTH RATING:

HEALTHY

FORAGE PRODUCTION(KG/HA)

$\begin{array}{lc}\text { GRASS } & 18 \\ \text { FORBS } & 238 \\ \text { SHRUBS } & 848 \\ \text { TOTAL } & 1104\end{array}$




\section{DMD4. Sw/Beaked hazelnut/Moss (Picea glauca/Corylus cornuta/Moss)}

$\mathbf{n}=\mathbf{1}$ This is a mature white spruce forest which represents the climax or near climax vegetation for the area. The northerly aspect of this community type has probably protected the site from past disturbance by fires and allowed the community to undergo succession. The high canopy of spruce limits the light reaching the forest floor, limiting the growth of grasses and forbs. As a result, the forage productivity of this community type is very low. This community would be considered non-use.

\section{Plant COMPOSITION CANOpY COVER(\%) \\ MEAN RANGE CONST.}

TREES

WHITE SPRUCE

(Picea glauca)

PAPER BIRCH

(Betula papyrifera)

SHRUBS

HAZELNUT

(Corylus cornuta)

BOG CRANBERRY

(Vaccinium vitis-idaea)

PRICKLY ROSE

(Rosa acicularis)

FORBS

BEARBERRY

(Arctostapylos uva-ursi)

TWINFLOWER

(Linnaea borealis)

BASTARD TOADFLAX

(Geocaulon lividum)

STRAWBERRY

(Fragaria virginiana)

Mosses

MOSS SPP.

$\begin{array}{lll}60 & - & 100 \\ 5 & - & 100 \\ 12 & - & 100 \\ 6 & - & 100 \\ 6 & - & 100\end{array}$

- 100

$8 \quad-\quad 100$

$2 \quad-\quad 100$

$2 \quad-\quad 100$

$73 \quad-\quad 100$
ENVIRONMENTAL VARIABLES

MOISTURE REGIME:

MESIC

NUTRIENT REGIME:

MEDIUM

ELEVATION:

$606 \mathrm{M}$

SOIL DRAINAGE:

WELL

PERCENT SLOPE GRADIENT:

$5 \%$

ASPECT:

NORTHERLY

RANGELAND HEALTH RATING:

HEALTHY

FORAGE PRODUCTION(KG/HA)

$\begin{array}{ll}\text { GRASS } & 0 \\ \text { FORBS } & 132 \\ \text { SHRUBS } & 74 \\ \text { TOTAL } & 206\end{array}$

ECOLOGICALLY SUSTAINABLE STOCKING RATE NON-USE 


\section{DMD5. Aw-Sw/Rose/Marsh reedgrass \\ (Populus tremuloides-Picea glauca/Rosa acicularis/Calamagrostis canadensis)}

$\mathbf{n = 1}$ This community represents a highly productive aspen community that is succeeding to white spruce. The presence of tall forbs wild sarsaparilla and fireweed indicate a high nutrient regime and a light grazing regime. At present this community type has a good level of forage for domestic livestock.

\section{Plant COMPOSITION CANopy COVER(\%)}

MEAN RANGE CONST.

\section{TREES}

WHITE SPRUCE

(Picea glauca)

$55 \quad 50-60 \quad 100$

POPULUS TREMULOIDES

(Populus tremuloides) $\quad 53 \quad 35-70 \quad 100$

SHRUBS

PRICKLY ROSE

(Rosa acicularis)

WILD RED RASPBERRY

$\begin{array}{llll}\text { (Rubus idaeus) } & 8 & 0-15 & 50\end{array}$

BRISTLY BLACK CURRANT

(Ribes lacustre)

LOW BUSH CRANBERRY

$\begin{array}{llll}\text { (Viburnum edule) } & 8 & 6-10 & 100\end{array}$

FORBS

BUNCHBERRY

(Cornus canadensis) $\quad 4 \quad 0-8 \quad 50$

FIELD HORSETAIL

(Equisetum arvense) $\quad 2 \quad 0-3 \quad 50$

TALL LUNGWORT

$\begin{array}{llll}\text { (Mertensia paniculata) } & 4 & 1-7 & 100\end{array}$

WILD SARSAPARILLA

(Aralia nudicaulis)

DEWBERRY

(Rubus pubscens)

FIREWEED

(Epilobium angustifolium)2

GRASSES

MARSH REEDGRASS

(Calamagrostis canadensis) 17

\section{ENVIRONMENTAL VARIABLES}

MOISTURE REGIME:

MESIC-SUBHYGRIC

NUTRIENT REGIME:

MEDIUM TO RICH

ELEVATION:

455-600(527) M

SOIL DRAINAGE:

WELL TO MODERATELY WELL

RANGELAND HEALTH RATING:

HEALTHY

FORAGE PRODUCTION(KG/HA)

$\begin{array}{ll}\text { GRASS } & 468 \\ \text { FORBS } & 534 \\ \text { SHRUBS } & 440 \\ \text { TOTAL } & 1442\end{array}$

ECOLOGICALLY SUSTAINABLE STOCKING

RATE

$1.3 \mathrm{HA} / \mathrm{AUM}(0.3 \mathrm{AUM} / \mathrm{AC})$ 


\section{DMD6. Aw-Pb-Sw/Willow/Wild sarsaparilla}

(Populus tremuloides-Populus balsamifera-Picea glauca/Salix spp./Aralia nudicaulis)

$\mathrm{n}=1$ This community type has similar moisture and nutrient conditions to the $\mathrm{Aw}-\mathrm{Pb}$ and $\mathrm{Pb} / \mathrm{Red}$ osier dogwoodRose community types, but this community is successionally more advanced. The abundance of tall shrubs limits the amount of light reaching the forest floor, which limits forage production. As a result, this community type would be considered non-use or marginal secondary range for domestic livestock.

\section{PlaANT COMPOSITION CANOPY COVER(\%) \\ MEAN RANGE CONST.}

TREES

ASPEN

(Populus tremuloides) 35

WHITE SPRUCE

(Picea glauca)

PAPER BIRCH

(Betula papyrifera)

BALSAM POPLAR

$\begin{array}{llll}\text { (Populus balsamifera) } & 25 & - & 100\end{array}$

SHRUBS

GREEN ALDER

(Alnus crispa)

WILLOW

(Salix spp.)

LOW BUSH CRANBERRY

(Viburnum edule)

PRICKLY ROSE

(Rosa acicularis)

FORBS

WILD SARSAPARILLA

(Aralia nudicaulis)

BISHOP's CAP

(Mitella nuda)

CANADA VIOLET

(Viola canadensis)

LADY FERN

(Athyrium filix-femina)

DEWBERRY

(Rubus pubescens)
15

10

45

25

10

10

13

13

$11 \quad-\quad 100$

$11 \quad-\quad 100$

$-\quad 100$

4

\section{ENVIRONMENTAL VARIABLES}

MOISTURE REGIME:

SUBHYGRIC

NUTRIENT REGIME:

$\mathrm{RICH}$

ELEVATION:

$606 \mathrm{M}$

SOIL DRAINAGE:

MODERATELY WELL

PERCENT SLOPE GRADIENT:

$20 \%$

ASPECT:

EAST

RANGELAND HEALTH RATING:

HEALTHY

FORAGE PRODUCTION(KG/HA)

$\begin{array}{ll}\text { GRASS } & 20 \\ \text { FORBS } & 400 \\ \text { SHRUBS } & 56 \\ \text { TOTAL } & 476\end{array}$

ECOLOGICALLY SUSTAINABLE STOCKING RATE

NON-USE 


\section{DMD7. Sw-Pb-Aw/Rose/Twinflower \\ (Picea glauca-Populus balsamifera-Populus tremuloides/Rosa acicularis/Linnaea borealis)}

$\mathbf{n}=\mathbf{1} \quad$ This community is similar to the previous described $\mathrm{Aw}-\mathrm{Pb}-\mathrm{Sw} / \mathrm{Willow} / \mathrm{Wild}$ sarsaparilla community type but is found on slightly drier sites with a poorer nutrient regime. Succession of this community type will likely be to a White spruce /Moss dominated community type. The thick overstory limits the growth of shrubs, forbs and grass. Consequently, there is little forage for domestic livestock. This community type would be considered non-use.

\section{Plant COMPOSITION CANOPY COVER(\%)} MEAN RANGE COSNT.

\section{TREES}

\section{WHITE SPRUCE}

(Picea glauca)

TREMBLING ASPEN

(Populus tremuloides)

BALSAM POPLAR

(Populus balsamifera)

\section{SHRUBS}

SNOWBERRY

\section{(Symphoricarpos}

occidentalis)

PRICKLY ROSE

(Rosa acicularis)

BRACTED HONEYSUCKLE

(Lonicera involcrata)

BUFFALO-BERRY

(Shepherdia canadensis)

FORBS

TWIN-FLOWER

\section{(Linnaea borealis)}

BUNCHBERRY

(Cornus canadensis)

WINTERGREEN

(Pyrola asarifolia)

DEWBERRY

(Rubus pubscens)

BISHOP'S CAP

(Mitella nuda)

$$
35
$$

20

30

100

100

100

0

Mosses

Moss SPP.

\section{3}

18

5

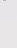

$71 \quad 100$

\section{ENVIRONMENTAL VARIABLES}

\section{MOISTURE REGIME:}

MESIC TO SUBHYGRIC

NUTRIENT REGIME:

MEDIUM

ELEVATION:

$606 \mathrm{M}$

SOIL DRAINAGE:

WELL TO MODERATELY WELL

RANGELAND HEALTH RATING:

HEALTHY

\section{FORAGE PRODUCTION(KG/HA)}

$\begin{array}{lc}\text { GRASS } & 16 \\ \text { FORBS } & 112 \\ \text { SHRUBS } & 108 \\ \text { TOTAL } & 236\end{array}$

ECOLOGICALLY SUSTAINABLE STOCKING RATE NON-USE 


\section{DMD8. Sb/Willow/Moss \\ (Picea mariana/Salix spp./Moss)}

$\mathbf{n}=\mathbf{2}$ This community type is part of the poor fen ecosite (Beckingham and Archibald 1996) because it has an intermediate nutrient regime between the bog and rich fen ecosites. Drainage on this community type is poor to very poor, but has some movement of water through the site. This community type has a well developed shrub layer and the grass layer consists mainly of marsh reedgrass and sedge species. The productivity of this type is moderate, but the high water table limits access to domestic livestock. This community would be rated as non-use.

\section{Plant COMPOSITION Canopy Cover(\%)} MEAN RANGE CONST.

\section{TREES}

BLACK SPRUCE

(Picea mariana)

SHRUBS

WILLOW SPP.

(Salix spp.)

BOG BIRCH

(Betula glandulosa)

FORBS

STEMLESS RASPBERRY

(Rubus arctica)

HORSETAIL

(Equisetum arvense) $\quad 18 \quad 15-20 \quad 100$

BISHOP's CAP

(Mitella nuda)

GRASSES

MARSH REEDGRASS

(Calamagrostis canadensis)10 $\quad 9-11 \quad 100$

HAIR-LIKE SEDGE

(Carex capillaris)

Mosses

Moss SPP.
$15 \quad 14-16 \quad 100$

$35 \quad 20-50 \quad 100$

$17 \quad 8-25 \quad 100$

$4 \quad 2-5 \quad 100$

$6 \quad 1-10 \quad 100$

$5-10 \quad 100$

$99-100 \quad 100$

\section{ENVIRONMENTAL VARIABLES}

MOISTURE REGIME:

SUBHYDRIC

NUTRIENT REGIME:

MEDIUM

ELEVATION:

606-697(657) M

SOIL DRAINAGE:

POORLY

RANGELAND HEALTH RATING:

HEALTHY

FORAGE PRODUCTION(KG/HA)

$\begin{array}{ll}\text { GRASS } & 401 \\ \text { FORBS } & 89 \\ \text { SHRUBS } & 242 \\ \text { TOTAL } & 732\end{array}$

ECOLOGICALLY SUSTAINABLE STOCKING RATE NON-USE 


\section{DMD9. Sb-Lt/Labrador tea/Moss \\ (Picea mariana-Larix laricina/Ledum groenlandicum/Moss)}

$\mathbf{n}=\mathbf{3}$ This community type is very similar to the previously described community type, but the nutrient status is poorer. This community type appears to be related to the bog ecosite described by Beckingham and Archibald (1996). The bog ecosite commonly has organic soils consisting of slowly decomposing peat moss. This community type is considered non-use for livestock, due to the lack of forage and poor accessibility.

\section{PLANT COMPOSITION CANOPY COVER(\%)}

MEAN RANGE CONST.

TREES

LARCH

(Larix laricina)

BLACK SPRUCE

(Picea mariana)

\section{SHRUBS}

WILLOW SPP.

(Salix spp.)

LABRADOR TEA

(Ledum groenlandicum) 23

BOG BIRCH

(Betula glandulosa)

FORBS

DWARF BRAMBLE

(Rubus pedatus)

HORSETAIL

(Equisetum arvense)

DWARF SCOURING RUSH

(Equisetum scirpoides)

$10 \quad 1-15 \quad 100$

$30 \quad 10-60 \quad 100$

GRASSES

MARSH REEDGRASS

(Calamagrostis canadensis)3

GOLDEN SEDGE

(Carex aurea)

BEAKED SEDGE

(Carex rostrata)

FOWL BLUEGRASS

(Poa palustris)

Mosses

MOSS SPP.

$21 \quad 8-35 \quad 100$

$10-35 \quad 100$

$24 \quad 0-39 \quad 100$

$8 \quad 0-25 \quad 66$

$21 \quad 0-45 \quad 66$

$8 \quad 0-25 \quad 33$

$1-4 \quad 100$

$0-15 \quad 33$

$0-7 \quad 66$

$1 \quad 0-2 \quad 33$

$95 \quad 10-60 \quad 100$

\section{ENVIRONMENTAL VARIABLES}

MOISTURE REGIME:

SUBHYDRIC

NUTRIENT REGIME:

VERY POOR

ELEVATION:

576-606 M

SOIL DRAINAGE:

POORLY

RANGELAND HEALTH RATING:

HEALTHY

\section{FORAGE PRODUCTION(KG/HA)}

$\begin{array}{ll}\text { GRASS } & 10 \\ \text { FORBS } & 40 \\ \text { SHRUBS } & 50 \\ \text { TOTAL } & 100\end{array}$

ECOLOGICALLY SUSTAINABLE STOCKING RATE NON-USE 


\section{DMD10. Sw-Aw/Low bush Cranberry \\ (Picea glauca-Populus tremuloides/Viburnum edule)}

$\mathbf{n}=\mathbf{5}$ This community is similar to community DMD5 Aw-Sw, but is successional more advanced. As succession continues in the absence of disturbance on these sites there will be a corresponding drop in forage production. A spruce dominated forest generally produces about $1 / 3$ of an undisturbed deciduous dominated community type. This community should be rated as secondary range.

\section{Plant COMPOSITION CANopy COVER(\%)}

MEAN RANGE CONST.

TREES

WHITE SPRUCE

(Picea glauca)

POPULUS TREMULOIDES

(Populus tremuloides) 14

SHRUBS

PRICKLY ROSE

(Rosa acicularis)

WILD RED RASPBERRY

(Rubus idaeus) 5

RED OSIER DOGWOOD

(Cornus stolonifera)

LOW BUSH CRANBERRY

(Viburnum edule)

FORBS

BUNCHBERRY

(Cornus canadensis) 3

FIELD HORSETAIL

(Equisetum arvense) 1

TALL LUNGWORT

(Mertensia paniculata) 1

WILD SARSAPARILLA

(Aralia nudicaulis)

DEWBERRY

(Rubus pubscens)

FIREWEED

(Epilobium angustifolium)2

GRASSES

MARSH REEDGRASS

(Calamagrostis canadensis)3

$28 \quad 20-40 \quad 100$

$1-30 \quad 100$

3-10 100

$0-10 \quad 80$

$0-30 \quad 80$

$1-10 \quad 100$

$0-10 \quad 80$

$0-3 \quad 60$

$1-3 \quad 100$

$0-30 \quad 80$

$1-3 \quad 100$

$0-3 \quad 80$

$0-10 \quad 80$

\section{ENVIRONMENTAL VARIABLES}

MOISTURE REGIME: MESIC-SUBHYGRIC

NUTRIENT REGIME: MEDIUM TO RICH

ELEVATION: 455-600(527) M

SOIL DRAINAGE:

WELL TO MODERATELY WELL

RANGELAND HEALTH RATING:

HEALTHY

FORAGE PRODUCTION(KG/HA)

GRASS $\quad 400$

FORBS $\quad 250$

SHRUBS $\quad 500$

TOTAL $1150 *$ ESTIMATE

ECOLOGICALLY SUSTAINABLE STOCKING RATE

$1.6 \mathrm{HA} / \mathrm{AUM}(0.25 \mathrm{AUM} / \mathrm{AC})$ 


\section{DMD11. Sw/Moss \\ (Picea glauca/Moss spp.)}

$\mathbf{n}=\mathbf{1}$ This community is similar to community DMD10 Sw-Aw, but is successional more advanced. As succession continues in the absence of disturbance on these sites there will be a corresponding drop in forage production. A spruce dominated forest generally produces about $1 / 3$ of an undisturbed deciduous and mixed wood dominated community types. This community should be rated as non-use.

\section{Plant COMPOSITION Canopy Cover(\%)} MEAN RANGE CONST.

\section{TREES}

WHITE SPRUCE

(Picea glauca)

\section{SHRUBS}

PRICKLY ROSE

(Rosa acicularis)

BRACTED HONEYSUCKLE

(Lonicera involcrata) 3

RED OSIER DOGWOOD

(Cornus stolonifera) 3

LOW BUSH CRANBERRY

(Viburnum edule) 1

FORBS

BUNCHBERRY

(Cornus canadensis) 1

FIELD HORSETAIL

(Equisetum arvense)

TWINFLOWER

(Linnaea borealis) 10

DEWBERRY

(Rubus pubscens)

GRASSES

PURPLE OATGRASS

(Schizachne purpurascens)3

\section{ENVIRONMENTAL VARIABLES}

MOISTURE REGIME: MESIC-SUBHYGRIC

NUTRIENT REGIME: MEDIUM TO RICH

ELEVATION: $600 \mathrm{M}$

SOIL DRAINAGE: WELL TO MODERATELY WELL

RANGELAND HEALTH RATING: HEALTHY

\section{FORAGE PRODUCTION(KG/HA)}

$\begin{array}{ll}\text { GRASS } & 10 \\ \text { FORBS } & 100 \\ \text { SHRUBS } & 100 \\ \text { TOTAL } & 210^{*} \text { ESTIMATE }\end{array}$

$$
\begin{gathered}
\text { ECOLOGICALLY SUSTAINABLE STOCKING } \\
\text { RATE } \\
\text { NON-USE }
\end{gathered}
$$




\section{DMD12. Sw-Bw/Raspberry \\ (Picea glauca-Betula papyrifera/Rubus ideaus)}

$\mathbf{n = 1}$ This community type was described near Astotin Lake in Elk Island National Park. It represents a site that has had historic beaver activity and since has undergone succession to a spruce dominated community. Cutting of the adjacent tree canopy and the increased moisture around the dam favours the growth of paper birch and raspberry. Both species are early successional and will rapidly dominate a site after disturbance. This community occupies small areas adjacent to the ponds and sloughs and therefore will contribute little to the overall carrying capacity of a lease. It should be rated as non-use.

\section{PlaAT COMPOSITION CANOPY COVER(\%) \\ MEAN RANGE CONST.}

TREES

WHITE SPRUCE

(Picea glauca)

PAPER BIRCH

(Betula papyrifera) 20

SHRUBS

PRICKLY ROSE

(Rosa acicularis)

BRACTED HONEYSUCKLE

(Lonicera involucrata) 3

RASPBERRY

(Rubus idaeus)

LOW BUSH CRANBERRY

(Viburnum edule) 3

FORBS

WILD SARSAPARILLA

(Aralia nudicaulis) 10

HEMP NETTLE

(Galeopsis tetrahit) $\quad 10$

FIREWEED

(Epilobium angustifolium) 3

SHOWY ASTER

(Aster conspicuus)

GRASSES

QUACKGRASS

(Agropyron repens) 3

SMOOTH BROME

(Bromus inermis)
100

$-\quad 100$
$-\quad 10$

100

100

100

100

$-\quad 100$

100

100

100

100

100

100

\section{ENVIRONMENTAL VARIABLES}

MOISTURE REGIME: MESIC-SUBHYGRIC

NUTRIENT REGIME: MEDIUM

ELEVATION:

$600 \mathrm{M}$

SOIL DRAINAGE:

WELL TO MODERATELY WELL

RANGELAND HEALTH RATING:

HEALTHY

FORAGE PRODUCTION(KG/HA)

TOTAL $850 *$ ESTIMATE

ECOLOGICALLY SUSTAINABLE STOCKING RATE

$2.1 \mathrm{HA} / \mathrm{AUM}(0.2 \mathrm{AUM} / \mathrm{AC})$ 


\section{DMD13. Sw-Pb/Red osier dogwood \\ (Picea glauca-Populus balsamifera/Cornus stolonifera)}

$\mathbf{n}=\mathbf{6}$ This community is similar to community $\mathrm{DMC} 8 \mathrm{~Pb}-\mathrm{Aw} / \mathrm{Red}$ osier dogwood, but is successional more advanced. As succession continues in the absence of disturbance on these sites there will be a corresponding drop in forage production. A spruce dominated forest generally produces about $1 / 3$ of an undisturbed deciduous dominated community type. This community should be rated as secondary range.

\section{Plant COMPOSITION Canopy Cover(\%)}

MEAN RANGE CONST.

\section{TREES}

WHITE SPRUCE

(Picea glauca) 23

BALSAM POPLAR

(Populus balsamifera) 30

SHRUBS

PRICKLY ROSE

RED OSIER DOGWOOD

(Cornus stolonifera)

RIVER ALDER

(Alnus tenuifolia)

LOW BUSH CRANBERRY

(Viburnum edule)

FORBS

WILD SARSAPARILLA

HORSETAIL

(Equisetum arvense)

STAR FLOWERED SOLOMON SEAL

(Smilacina stellata)

BUNCHBERRY

(Cornus canadensis)

GRASSES

MARSH REEDGRASS

(Calamagrostis canadensis)2 $\quad 0-3 \quad 67$

KENTUCKY BLUEGRASS

(Poa pratensis)

3

REDTOP

(Agrostis stolonifera) 6
$1-40 \quad 100$

$20-60 \quad 100$

3-20 $\quad 100$

$3-20 \quad 100$

$3-30 \quad 100$

$0-10 \quad 67$

$0-10 \quad 67$

$1-10 \quad 100$

$1-3 \quad 100$

$0-30 \quad 83$

$0-10 \quad 83$

$0-20 \quad 83$

\section{ENVIRONMENTAL VARIABLES}

MOISTURE REGIME: SUBHYGRIC

NUTRIENT REGIME: $\mathrm{RICH}$

ELEVATION: $600 \mathrm{M}$

SOIL DRAINAGE:

WELL TO MODERATELY WELL

RANGELAND HEALTH RATING:

HEALTHY

FORAGE PRODUCTION(KG/HA)

TOTAL $\quad 620 *$ EsTIMATE

ECOLOGICALLY SUSTAINABLE STOCKING

RATE

NON-USE 


\section{DMD14. Sw/Horsetail \\ (Picea glauca/Equisetum arvense)}

$\mathbf{n}=5 \quad$ This community type is wet and nutrient rich. These sites are commonly found on fluvial or glaciolacustrine parent materials where flooding or seepage enhances the substrate nutrient supply. With high water tables, wet soil conditions organic matter tends to accumulate which favours the growth of horsetails. Generally horsetails are unpalatable to livestock and the wet ground conditions limit access. Consequently, this community type should be rated as non-use.

\section{Plant COMPOSITION CANopy Cover(\%)} MEAN RANGE CONST.

TREES

WHITE SPRUCE

(Picea glauca)

BALSAM POPLAR

(Populus balsamifera)

PAPER BIRCH

$\begin{array}{llll}\text { (Betula papyrifera) } & 9 & 0-40 & 80\end{array}$

SHRUBS

PRICKLY ROSE

(Rosa acicularis) $\quad 3 \quad 1-10 \quad 100$

RED OSIER DOGWOOD

(Cornus stolonifera)

BRACTED HONEYSUCKLE

(Lonicera involucrata)

LOW BUSH CRANBERRY

(Viburnum edule)

\section{FORBS}

DEWBERRY

(Rubus pubescens)

HORSETAIL

(Equisetum arvense) 32

BISHOP'S CAP

(Mitella nuda)

BUNCHBERRY

(Cornus canadensis)

GRASSES

MARSH REEDGRASS

(Calamagrostis canadensis)1

NODDING WOOD REED

(Cinna latifolia)

1
$20-60 \quad 100$

$0-10 \quad 60$

$0-10 \quad 80$

$0-3 \quad 80$

$0-3 \quad 60$

$1-3 \quad 100$

$30-40 \quad 100$

$0-3 \quad 80$

$0-10 \quad 80$

$0-3 \quad 60$

$0-3 \quad 40$

\section{ENVIRONMENTAL VARIABLES}

MOISTURE REGIME: HYGRIC

NUTRIENT REGIME:

$\mathrm{RICH}$

ELEVATION:

$600 \mathrm{M}$

SOIL DRAINAGE: POOR TO MODERATELY WELL

RANGELAND HEALTH RATING:

HEALTHY

FORAGE PRODUCTION(KG/HA)

TOTAL $\quad 560 *$ ESTIMATE

ECOLOGICALLY SUSTAINABLE STOCKING

RATE

NON-USE 


\section{CENTRAL MIXEDWOOD SUBREGION}

This subregion is the largest in the province covering over $210,000 \mathrm{~km}^{2}$ or nearly $32 \%$ of the province (Strong and Leggat 1992)( Map 1). Mean annual summer temperatures average $13.5^{\circ} \mathrm{C}$ and winter temperatures average $-13{ }^{\circ} \mathrm{C}$, which is somewhat colder than the adjacent Dry Mixedwood subregion. Annual precipitation averages $397 \mathrm{~mm}$ of precipitation which is wetter than the Dry Mixedwood.

The modal plant communities are vegetated by aspen and balsam poplar with understories composed of a variety of herbs and deciduous shrubs. White spruce and balsam fir are the climatic climax species but are not well represented because of the frequent occurrence of fire. On dry, well drained, coarse-textured soils jack pine dominates and the poorly drained sites are dominated by black spruce, willows and sedge species. These reference communities are very similar to the Dry Mixedwood subregion, but the drier conditions of the Dry Mixedwood favours the formation of a number of native grassland communities, which are not found in the Central Mixedwood. Table 6 outlines the ecological sites, ecological site phases and reference range plant community types in the Central Mixedwood subregion. There are a number of new ecological sites (ecosites) and ecological site phases (ecosite phases) which are not found in the guide "Ecosites of Northern Alberta" (Beckingham and Archibald 1996) and they are outlined here. The new ecosite includes (aa) grass/shrubland and the new ecosite phases include (aa1) plains wormwood, (d4)shrubland, (e4) shrubland, and (j3) grassland poor fen (Table 6). The "Successional communities" or "Harvesting and Fire succession" categories (Table 1 and 6) outline the successional sequence the community type will undergo with increased grazing pressure or with harvesting or fire disturbance. There are a number of ecological site phase tables which summarize these successional communities. These include (d1a) grazed Aw, (d1c) burned Aw, (d3c) burned Sw, and (k2a) grazed willow.

The 55 range plant communities described in the Central Mixedwood subregion are arranged into 4 categories. These include:

\section{Central Mixedwood subregion}

CMA. Native grass and shrubland

CMB. Tame grasslands

CMC. Deciduous community types

CMD. Mixedwood and Conifer community types
18 types

7 types

18 types

12 types

The dominant plant species, canopy cover, environmental conditions, forage production and grazing capacity (when available) are outlined for each community type. 


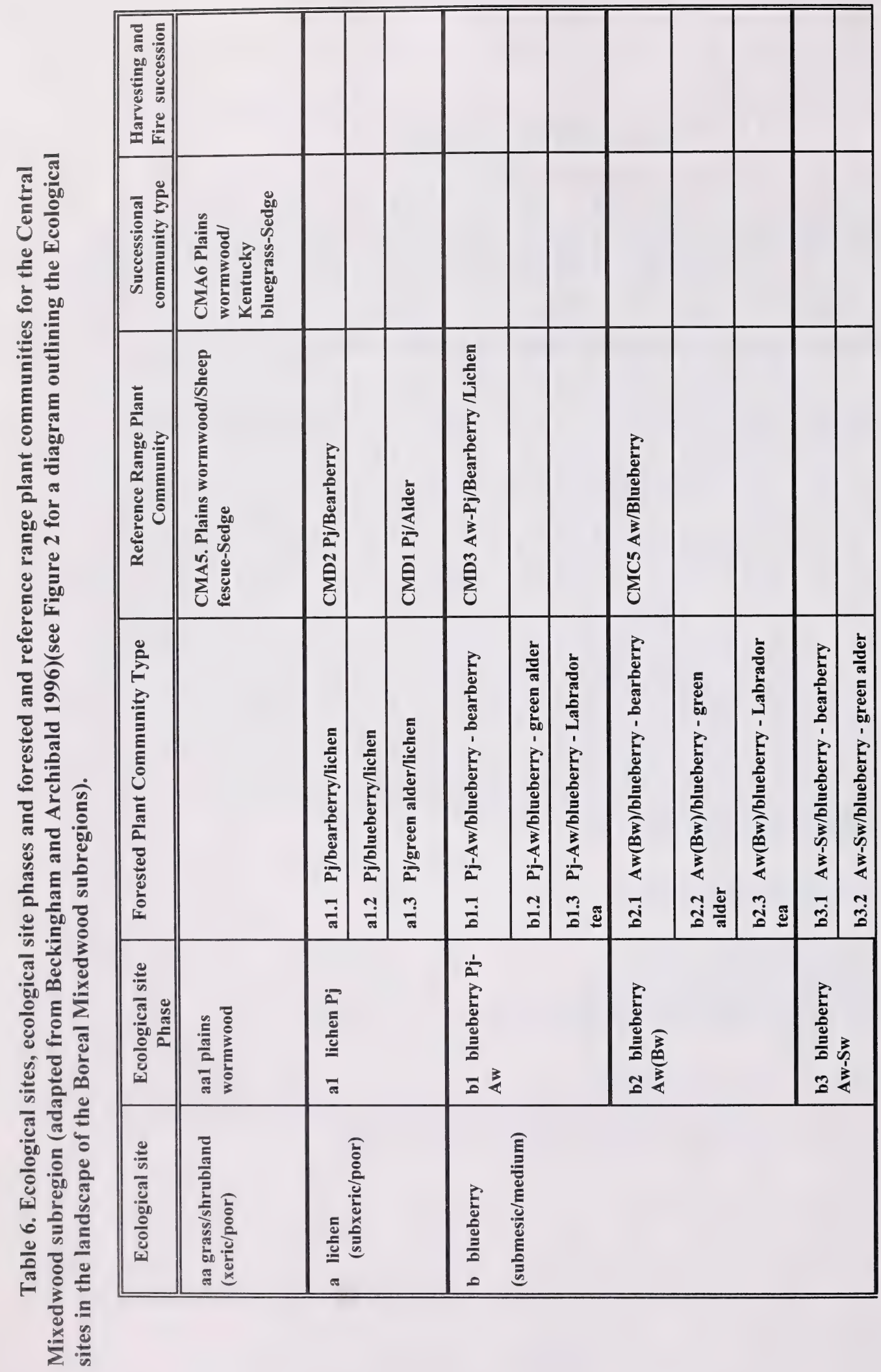

$\stackrel{\infty}{ \pm}$ 


\begin{tabular}{|c|c|c|c|c|c|c|c|c|c|c|c|c|}
\hline 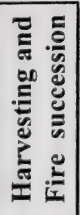 & & & & & & & & & & & & \\
\hline 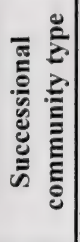 & & & & & & & & & & & 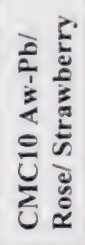 & 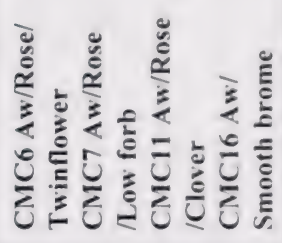 \\
\hline 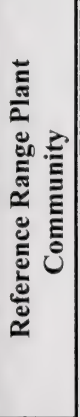 & & & & & & & 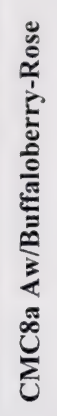 & 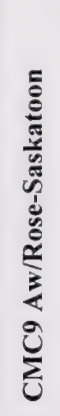 & 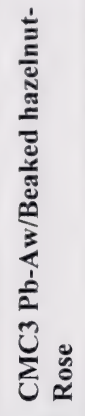 & 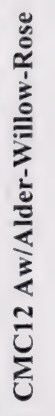 & & 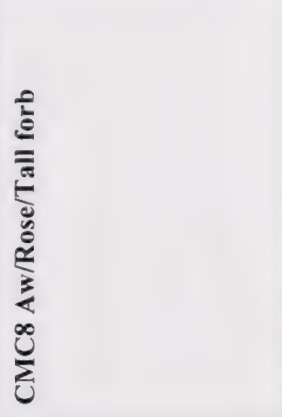 \\
\hline 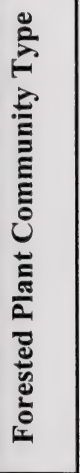 & 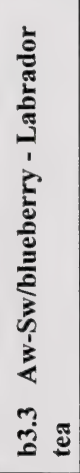 & 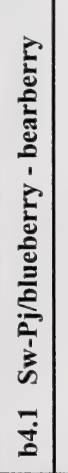 & 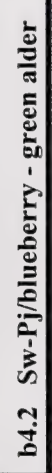 & 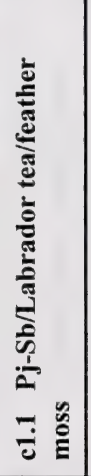 & 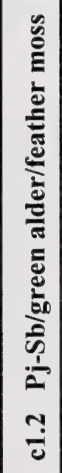 & 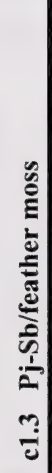 & 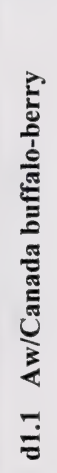 & 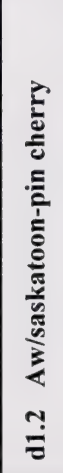 & 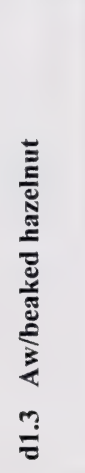 & 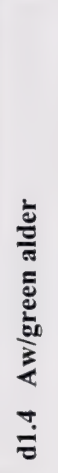 & 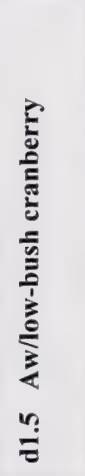 & 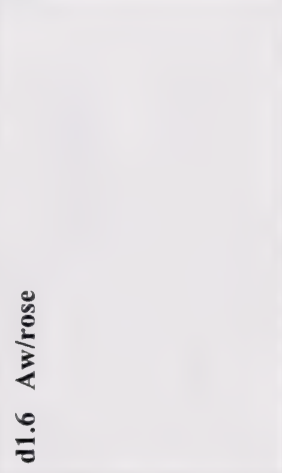 \\
\hline 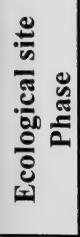 & & 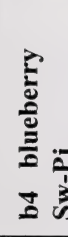 & & 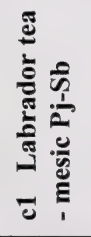 & & & 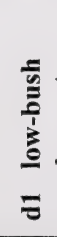 & & & & & \\
\hline 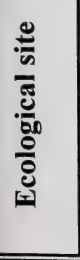 & & & & : & & & $\frac{\bar{c}}{\stackrel{0}{0}}$ & & & & & \\
\hline
\end{tabular}




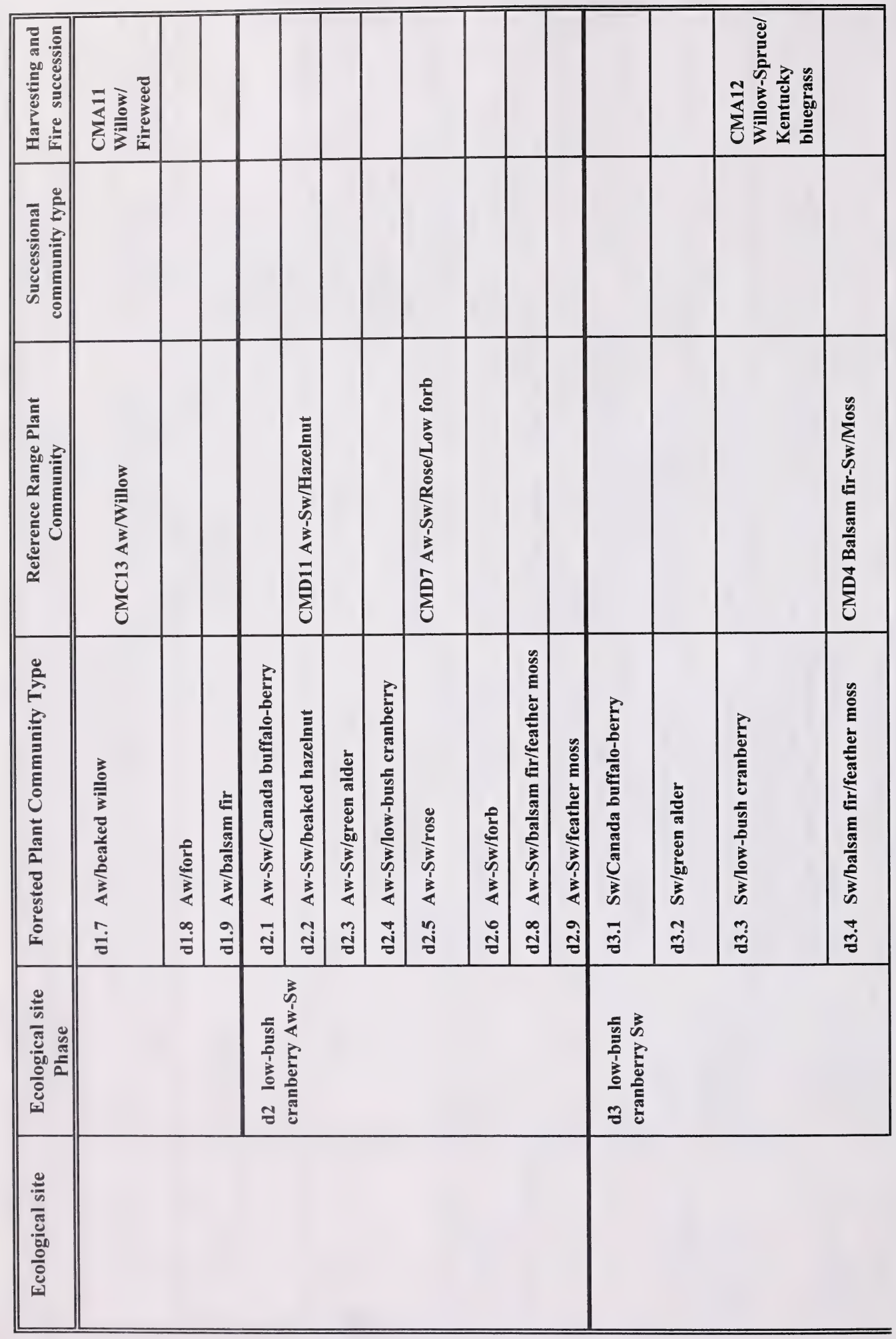

๓ 


\begin{tabular}{|c|c|c|c|c|c|c|c|c|c|c|c|c|c|c|}
\hline 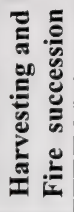 & & & & & & & & & & & & & & \\
\hline 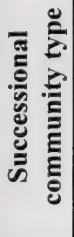 & 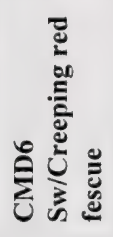 & 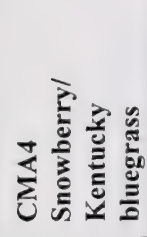 & & & & & & & & & & & & \\
\hline 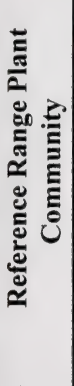 & 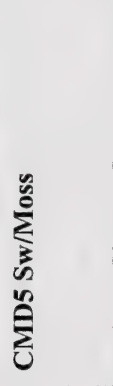 & & 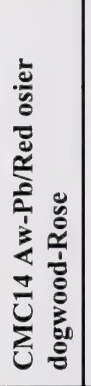 & 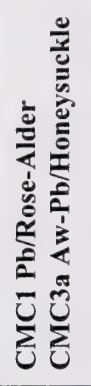 & 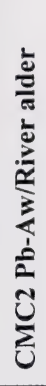 & & & & & & & & & \\
\hline 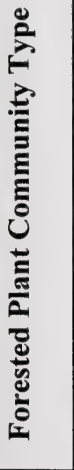 & 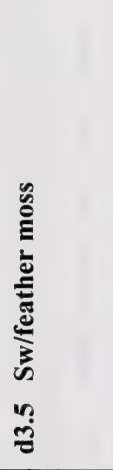 & & 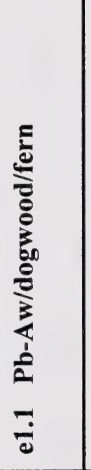 & 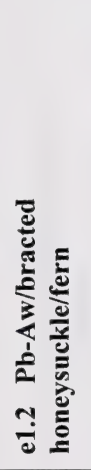 & 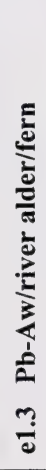 & 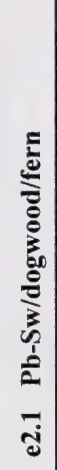 & 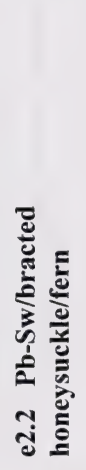 & 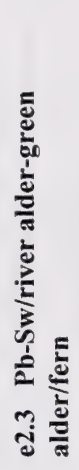 & 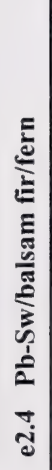 & 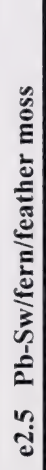 & 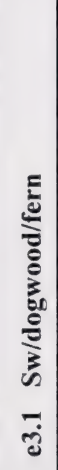 & 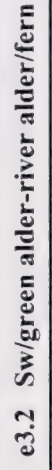 & 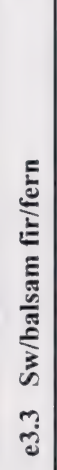 & 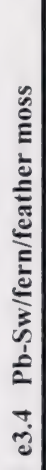 \\
\hline 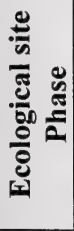 & & 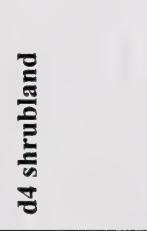 & 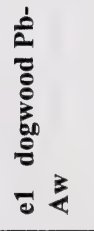 & & & 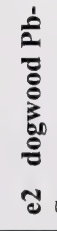 & & & & & 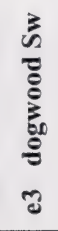 & & & \\
\hline 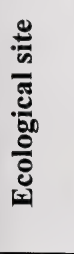 & & & 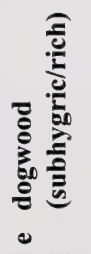 & & & & & & & & & & & \\
\hline
\end{tabular}




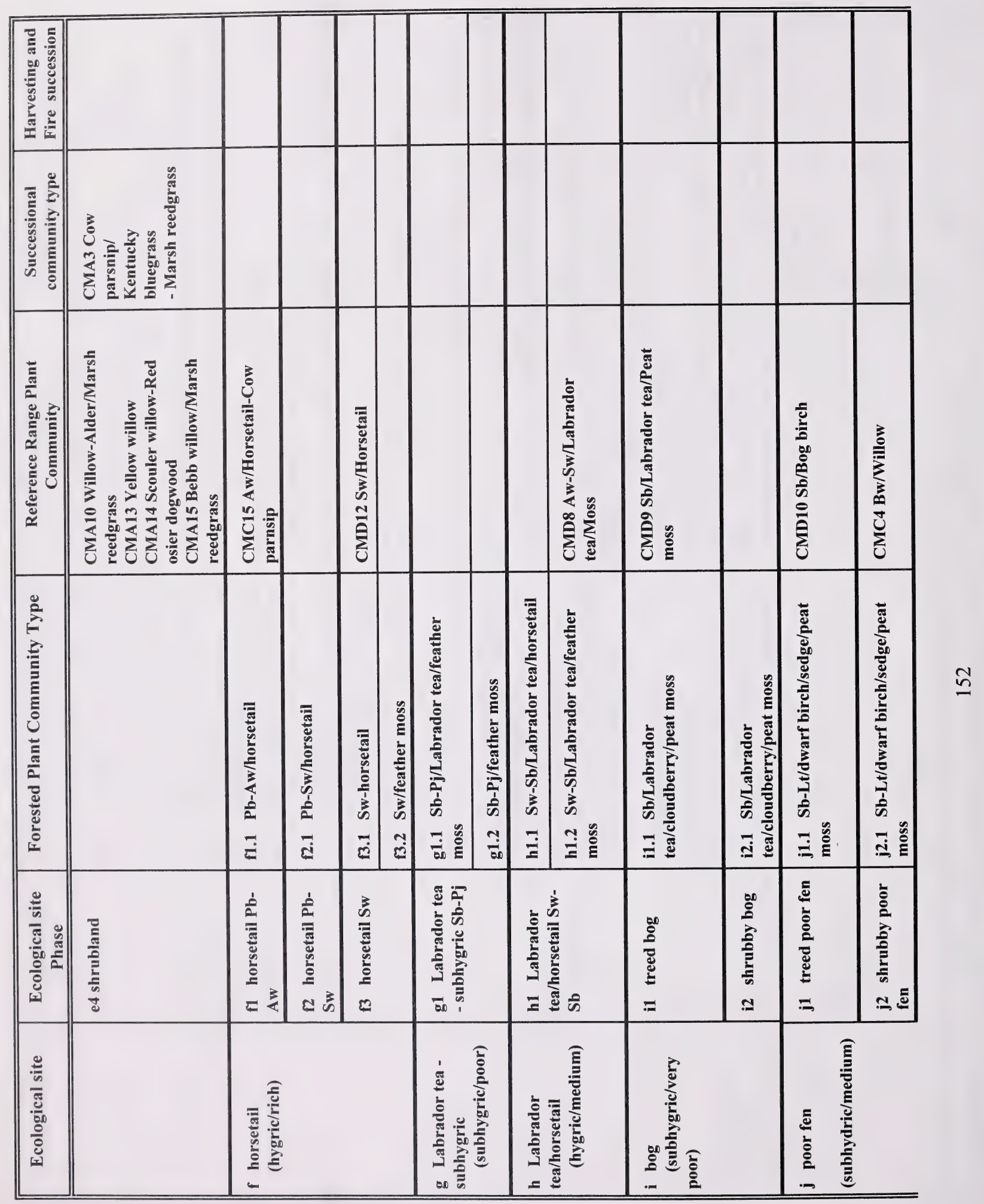




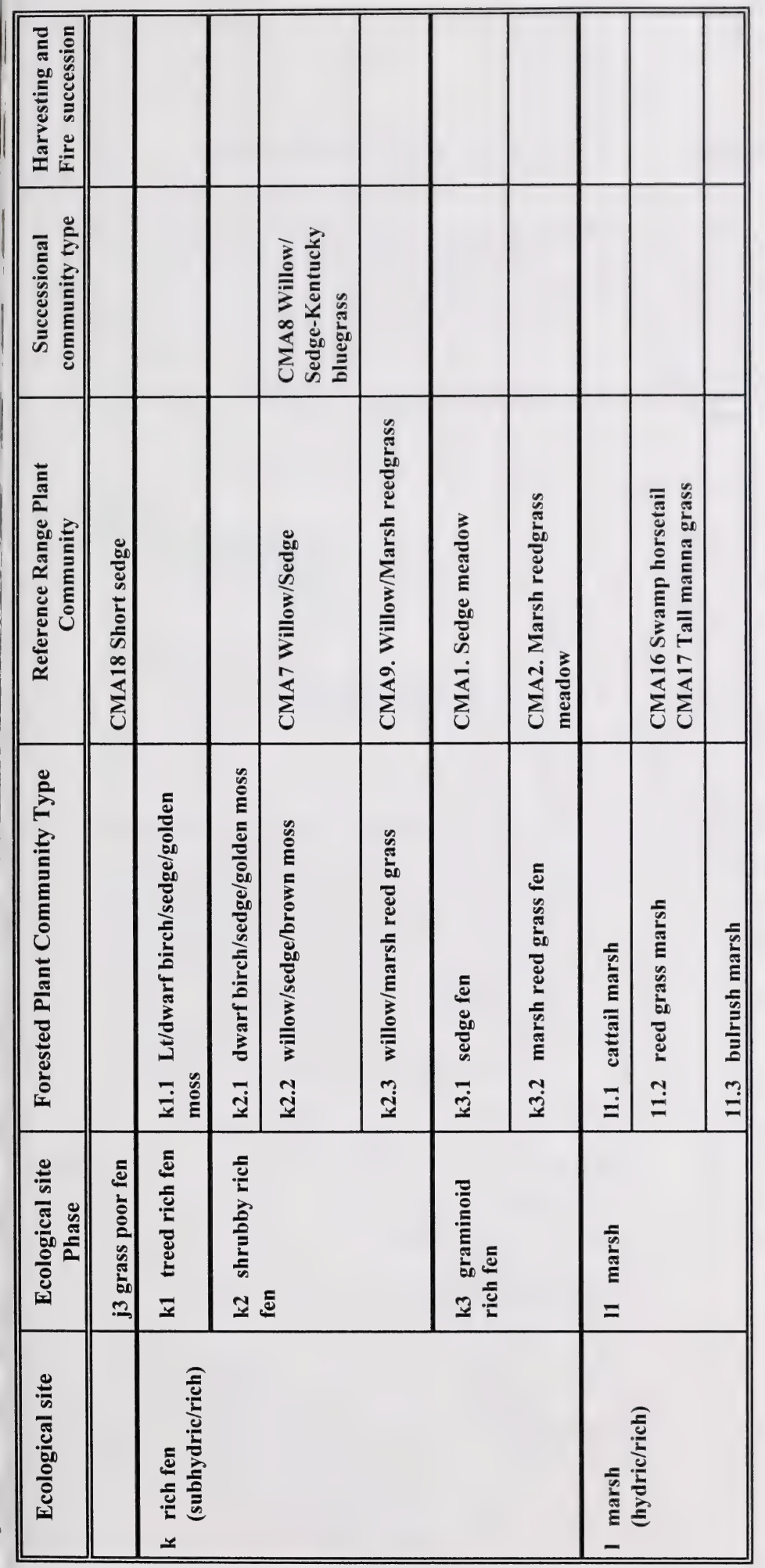


Figure 6. Edatopic grid for the Central Mixedwood subregion.

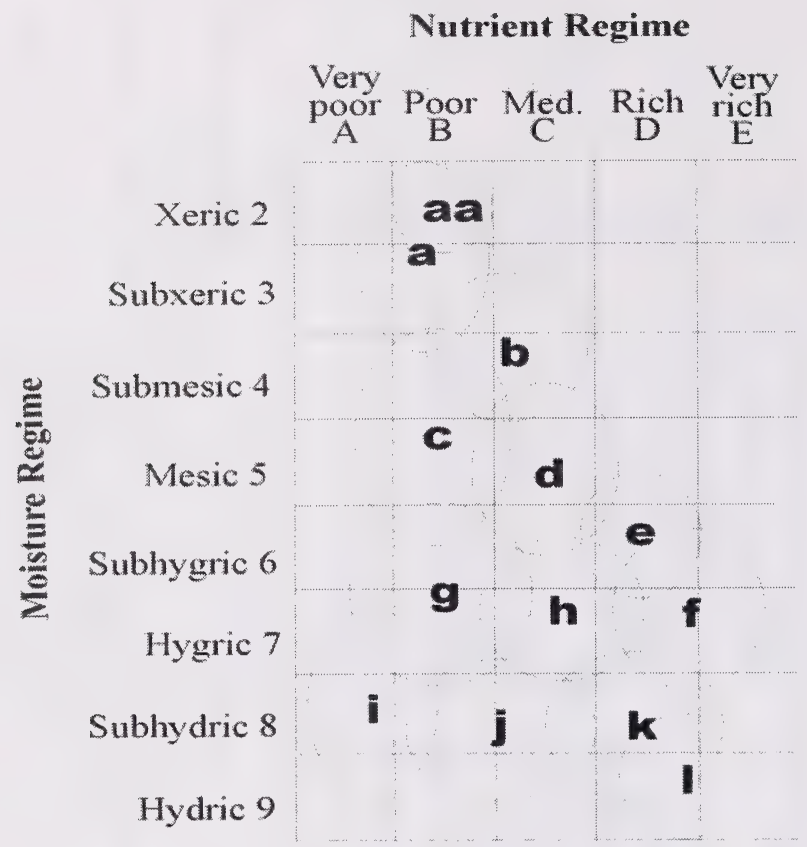

Ecological sites for the Central Mixedwood subregion

aa grassland
(xeric/poor)
a lichen
(subxeric/poor)
b blueberry
(submesic/medium)
c Labrador tea-mesic
(mesic/poor)
d low-bush cranberry
(mesic/medium)
e dogwood
(subhygric/rich)

$\mathrm{f}$ horsetail

(hygric/rich)

g Labrador tea-subhygric

(subhygric/poor)

h Labrador tea/horsetail

(hygric/medium)

i bog

(subhygric/very poor)

j poor fen

(subhydric/medium)

$\mathrm{k}$ rich fen

(subhydric/rich)

1 marsh

(hydric/rich) 


\section{GENERAL DESCRIPTION}

This ecosite is associated with small grassy openings within Jack pine and aspen forests. This site has dry conditions, with rapidly drained, nutrient poor soils. The parent materials are generally coarse textured eolian, glacialfluvial or fluvial eolian in origin. The high insolation and dry site conditions favour the growth of grassland species. These include Northern ricegrass, slender wheatgrass, Sedge, bearberry and plains wormwood In the moister sites (lower slope positions) aspen and shrubs (saskatoon, rose) are quite common.

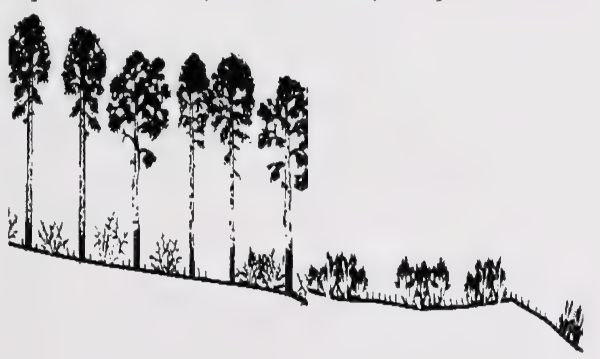

\section{SUCCESSIONAL RELATIONSHIPS}

Due to the nature of the site grasslands often remain the climax vegetation on these sites. In the moister lower slope positions shrubs often dominate the site with succession to aspen and spruce. On the drier hilltops and midslopes grasslands dominated by plains wormwood and northern ricegrass usually represent the climax vegetation. Heavy grazing pressure on the grasslands can often lead to a degraded site that is dominated by kentucky bluegrass, dandelion, and sedge species.

\section{INDICATOR SPECIES}

\section{Blueberry}

Rose

Saskatoon

Plains wormwood

Bearberry

Strawberry

Fleabane

Clover

Sheep fescue

Northern ricegrass

Slender wheatgrass

Kentucky bluegrass

Hairy wildrye

\section{SITE CHARACTERISTICS}

Moisture regime: xeric, subxeric, submesic

Nutrient regime: poor, medium

Topographic position: crest, upper, mid to lower slope

Slope: $(0-2 \%)(5-10 \%)$

Aspect: south, southwest, west

\section{SOIL CHARACTERISTICS}

Organic thickness: $(0-2)$

Humus form: mor

Surface texture: SL, $L$

Effective texture: SL, S

Depth to Mottles/Gley: none

Drainage: rapid, well

Parent material: E, GF,FE,F

Soil subgroup: O.EB, E.DYB O.R

\section{ECOSITE PHASES}

aal Plains wormwood (4) 


\section{CHARACTERISTIC SPECIES}

Shrub

[3] Saskatoon

[1] Rose

[3] Blueberry

Forb

[2] Fleabane

[9] Plains wormwood*

[2] Low goldenrod

[1] Scouring rush

[ 1] Clover

[1] Common yarrow

Grasses

[9] Sedge species*

[4] Sheep fescue*

[1] Creeping red fescue

[17] Kentucky bluegrass

[4] Northern ricegrass*

[3] Purple oatgrass

\section{RANGE PLANT COMMUNITY TYPES}

DMA3. Plains wormwood/Sheep fescue-Sedge

DMA4. Plains wormwood/Kentucky bluegrass-Sedge

\section{SITE CHARACTERISTICS}

Moisture regime: xeric, subxeric

Nutrient regime: poor,

Topographic position: crest, upper slope, midslope Slope: $5-10 \%$

Aspect: westerly, southerly

\section{SOIL CHARACTERISTICS}

Organic thickness: $(0-2)$

Humus form: mor

Surface texture: S, SL

Effective texture: $\mathrm{S}$

Depth to Mottles/Gley: none

Drainage: rapid, well

Parent material: E, GF, FE 


\section{CHARACTERISTIC SPECIES}

Tree

[46] Aspen*

[10] Balsam poplar

[3] White spruce

Shrub

[4] Raspberry

[2] Low bush cranberry

[6] Snowberry

[ 14] Rose*

[6] Willow

Forb

[2] Northern bedstraw

[ 5] Strawberry*

[3] Yellow peavine

[3] Bunchberry*

[ 8] Twinflower*

[2] Wild lily-of-the-valley

[3] Dewberry

[4] Wintergreen

[1] Dandelion*

[2] Clover species*

\section{Grasses}

$\begin{array}{ll}\text { [ 4 ] } & \text { Marsh reedgrass* } \\ \text { [ 2 ] } & \text { Hairy wildrye } \\ \text { [ 1] } & \text { Purple oatgrass } \\ \text { [ 1 ] } & \text { Kentucky bluegrass* }\end{array}$

\section{SITE CHARACTERISTICS}

Moisture regime:, mesic

Nutrient regime: medium

Topographic position: mid, lower slope, level

Slope: $0-5 \%$

Aspect: variable

\section{SOIL CHARACTERISTICS}

Organic thickness: (6-15), (0-5)

Humus form: mor, raw moder

Surface texture: SiL, SL, S, L

Effective texture: C, SiC, CL, SCL, SiCL

Depth to Mottles/Gley: none, (0-25)

Drainage: well, mod. well, imperfect

Parent material: GF, M, GL

Soil subgroup: O.GL, GR.GL, GL.GL

\section{RANGE PLANT COMMUNITY TYPES}

CMC6. Aw/Rose/Twinflower

CMC7. Aw/Rose/Low forb

CMC10. Aw- $\mathrm{Pb} /$ Rose/Strawberry

CMC11. Aw/Rose/Clover 
CMA11. Willow/Fireweed

\section{CHARACTERISTIC SPECIES}

Tree

[1] Aspen*

[1] White birch

Shrub

[1] Raspberry

[21] Willow

Forb

[ 37 ] Fireweed*

[2] Strawberry*

[2] Large leafed avens

[2] Common yarrow

[1] Harebell

\section{Grasses}

[ 19] Marsh reedgrass*

Mosses

[11] Moss species

\section{SITE CHARACTERISTICS}

Moisture regime:, mesic

Nutrient regime: medium

Topographic position: mid, lower slope, level

Slope: 0-5\%

Aspect: variable

\section{SOIL CHARACTERISTICS}

Organic thickness: (6-15), (0-5)

Humus form: mor, raw moder

Surface texture: $\mathrm{SiL}, \mathrm{SL}, \mathrm{S}, \mathrm{L}$

Effective texture: C, SiC, CL, SCL, SiCL

Depth to Mottles/Gley: none, (0-25)

Drainage: well, mod. well, imperfect

Parent material: GF, M, GL

Soil subgroup: O.GL, GR.GL, GL.GL 


\section{CHARACTERISTIC SPECIES}

\begin{tabular}{ll}
\hline Tree & \\
{$\left[\begin{array}{ll}8] & \text { Larch } \\
{[5]} & \text { Aspen } \\
{[25]} & \text { White spruce* }\end{array}\right.$}
\end{tabular}

Shrub

[6] Rose

[50] Willow

Forb

[ 22 ] Clover species*

[5] Strawberry

[6] Bishop's cap

[ 1] Common yarrow

[14] Dandelion*

[2] Canada thistle

\section{Grasses}

[1] Fringed brome

[ 77 ] Kentucky bluegrass*

\section{SITE CHARACTERISTICS}

Moisture regime:, mesic, subhygric, submesic

Nutrient regime: medium, rich, poor

Topographic position: mid, lower slope, level

Slope: (0-5), level, (6-9)

Aspect: variable

\section{SOIL CHARACTERISTICS}

Organic thickness: $(6-15),(0-5)$

Humus form: mor,

Surface texture: SiL, SL, S, CL, L

Effective texture: C, CL, SiC, SCL, SiCL

Depth to Mottles/Gley: none, (0-25)

Drainage: well, mod. well, imperfect

Parent material: GF, M, GL, GF/M, L

Soil subgroup: O.GL, BR.GL, GL.GL, E.EB 
Parent material: GF, F, GL

Soil subgroup: O.GL, GL.GL

\section{RANGE PLANT COMMUNITY TYPES}

CMA4. Snowberry/Kentucky bluegrass

\section{CHARACTERISTIC SPECIES}

Tree

[1] Aspen

Shrub

[9] Raspberry

[19] Snowberry*

[3] Rose

[5] Willow

Forb

[1] Northern bedstraw

[1] Strawberry

[1] Large leafed avens

[2] Cow parsnip

[1] Fireweed

[2] Common yarrow

[1] American vetch

[2] Canada thistle

[32] Dandelion*

[29] Clover species*

\section{Grasses}

[ 7 ] Marsh reedgrass*

[3] Sedge species

[7] Slender wheatgrass

[ 38 ] Kentucky bluegrass*

\section{SITE CHARACTERISTICS}

Moisture regime:, mesic, subhygric

Nutrient regime: medium, rich

Topographic position: level

Slope: 0-5\%

Aspect: variable

\section{SOIL CHARACTERISTICS}

Organic thickness: (0-5)

Humus form: moder

Surface texture: SiL, SL, L

Effective texture: SCL, SiCL, SiL

Depth to Mottles/Gley: none, (0-25)

Drainage: well, mod. well, 


\section{CHARACTERISTIC SPECIES}

Tree

[ 5] Paper birch

[1] White spruce

\section{Shrub}

[ 14] Raspberry

[ 5] Bracted honeysuckle

[4] Rose

[11] Green alder

[ 12] River alder

[39] Willow*

[ 10] Yellow willow

[ 10] Red osier dogwood

Forb

[1] Northern bedstraw

[1] Strawberry

[ 9] Horsetail*

[9] Cow parsnip*

[4] Fireweed

[4] Wild sarsaparilla

[2] American vetch

[ 5] Dandelion

\section{Grasses}
[ 29] Marsh reedgrass*
[ 5] Sedge species
[1] Slender wheatgrass
[3] Kentucky bluegrass

\section{SITE CHARACTERISTICS}

Moisture regime:, subhygric, mesic,

Nutrient regime: rich, medium,

Topographic position: level

Slope: $0-5 \%$

Aspect: variable

\section{SOIL CHARACTERISTICS}

Organic thickness: $(6-15),(0-5)$

Humus form: mor, raw moder

Surface texture: SiL, SL, L, S, SCL

Effective texture: $\mathrm{SiC}, \mathrm{C}, \mathrm{CL}, \mathrm{SiCL}, \mathrm{SCL}, \mathrm{S}$

Depth to Mottles/Gley: none, (0-25)

Drainage: imperfect, mod. well, poor, well

Parent material: GL, F

Soil subgroup: O.LG, O.G, GL.GL

\section{RANGE PLANT COMMUNITY TYPES}

CMA3. Cow parsnip/Kentucky bluegrass-Marsh reedgrass

CMA10. Willow-Alder/Marsh reedgrass

CMA13. Yellow willow

CMA14. Scouler willow-Red osier dogwood

CMA15. Bebb willow/Marsh reedgrass 


\section{RANGE PLANT COMMUNITY TYPES}

CMA8. Willow/Sedge/Kentucky bluegrass

\section{CHARACTERISTIC SPECIES}

Shrub

\section{[3] Rose}

[25 ] Willow*

Forb

[3] Mint

[2] Large leaved avens

[1] Dock

[5] Dandelion

[9] Clover species*

[2] Plantain

[3] Strawberry*

[4] Arrow-leaved coltsfoot

\section{Grasses}

[1] Marsh reedgrass

[ 44 ] Sedge species*

[3] Fowl bluegrass

[21 ] Kentucky bluegrass*

\section{SITE CHARACTERISTICS}

Moisture regime:, hydric, subhydric, hygric

Nutrient regime: rich, medium, very rich

Topographic position: level, depression

Slope: $0-5 \%$

Aspect: variable

\section{SOIL CHARACTERISTICS}

Organic thickness: $>80,(6-15),(0-5)$

Humus form: peatmor

Surface texture: fibric, C, mesic, SiL, humic

Effective texture: mesic, C, hC, fibric, $\mathrm{SiC}$, humic

Depth to Mottles/Gley: none, (0-25)

Drainage: very poor, poor

Parent material: O, GL, L

Soil subgroup: R.G, R.HG, TY.F, O.G 
i3 grassland $(n=1)$

CHARACTERISTIC SPECIES

Shrub

[1] Willow*

Forb

[1] Water hemlock

[1] Skull cap

\section{Grasses}

[10] Northern reedgrass*

[60] Short sedge

[ 20] Water sedge

\section{SITE CHARACTERISTICS}

Moisture regime:, subhydric

Nutrient regime: medium,

Topographic position: level

Slope: $0-5 \%$

Aspect: variable

\section{SOIL CHARACTERISTICS}

Organic thickness: $>80$

Humus form:

Surface texture: fibric

Effective texture: fibric

Depth to Mottles/Gley: not applicable

Drainage: very poor

Parent material: $O$

Soil subgroup: TY.F

\section{RANGE PLANT COMMUNITY TYPES}

CMA18. Short sedge 


\section{CENTRAL MIXEDWOOD SUBREGION}

\section{GRASSLAND AND SHRUBLAND COMMUNITY TYPES}

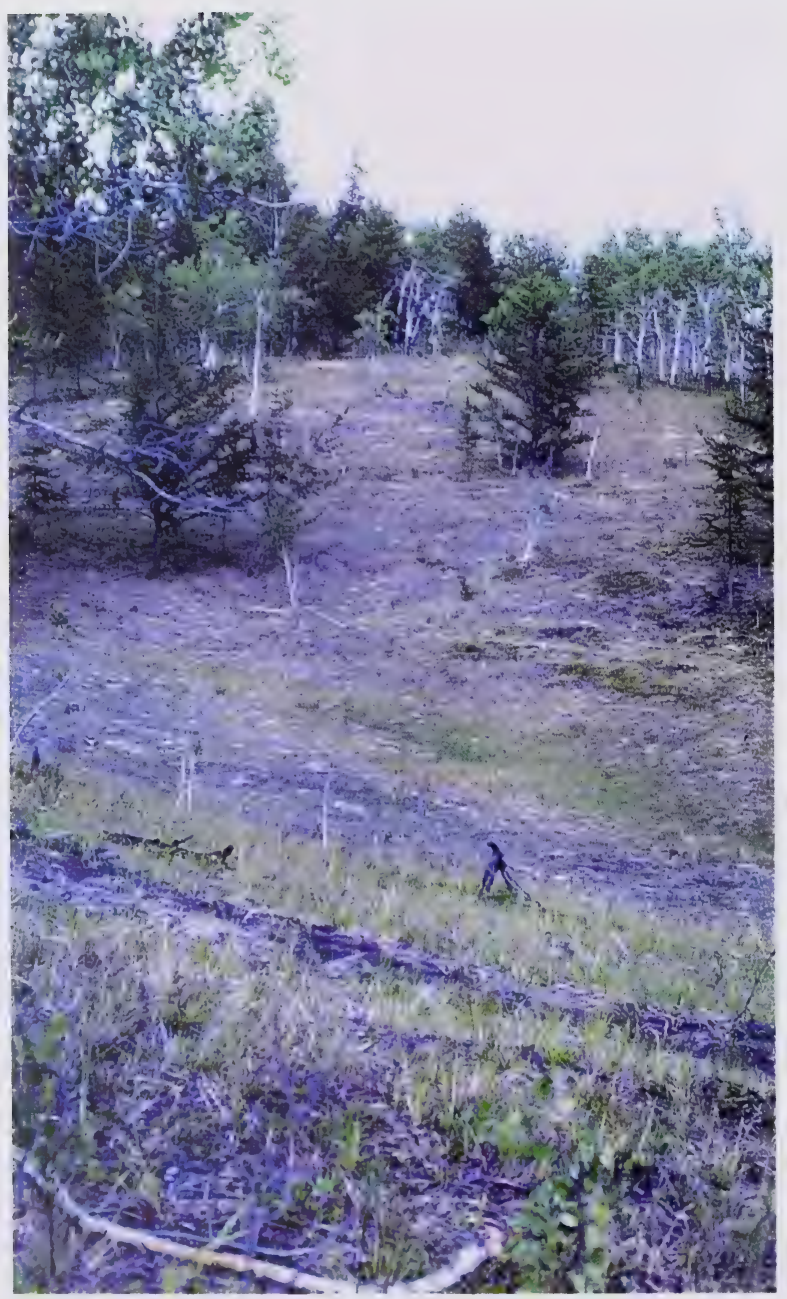

Photo 7. This picture represents the Plains wormwood/Sheep fescue-Sedge community type. This community type is common on dry sandy hills throughout the Central Mixedwood subregion. 


\section{CM - NATIVE GRASS AND SHRUBLAND COMMUNITIES}

Upland native grasslands are very rare in the Central Mixedwood subregion. The communities that have been described, occur on coarse textured, sandy soil, with xeric to subxeric moisture and poor nutrient regimes which lack tree cover. This includes the Plains wormwood/Sheep fescue-Sedge community type. This community type is usually found in association with jack pine dominated community types. Heavy grazing of this community type can lead to a Kentucky bluegrass-Sedge/Plains wormword dominated type on slightly moister sites. On level, gravelly, well-drained sites adjacent to streams and rivers a snowberry dominated community type is common. This community is extensively grazed by livestock to form the Snowberry/Kentucky bluegrass dominated type (Figure 3).

Wetter (subhydric/rich) sites are associated with sedge, swamp horestail, tall manna grass and marsh reedgrass dominated meadows. Sedge and swamp horsetail species are usually associated with the areas of free standing water, whereas, tall manna grass and marsh reedgrass dominate the better drained, drier edges. Willow will invade into these meadows to form the Willow/Sedge and Willow/Marsh reedgrass community types. Under grazing pressure these community types tended to be invaded by dandelion, clover and Kentucky bluegrass to form the Willow/Sedge-Kentucky bluegrass community type.

Fire is an important part of the ecology of the Central Mixedwood subregion. There are a number of shrubland community types which have a strong fire origin. These include the Willow-River alder/Marsh reedgrass, Willow/Fireweed and Willow-Spruce/ Kentucky bluegrass dominated community types. Other upland shrub communities which are found on nutrient rich, seepage areas include the Scouler and Bebb willow dominated communities. 

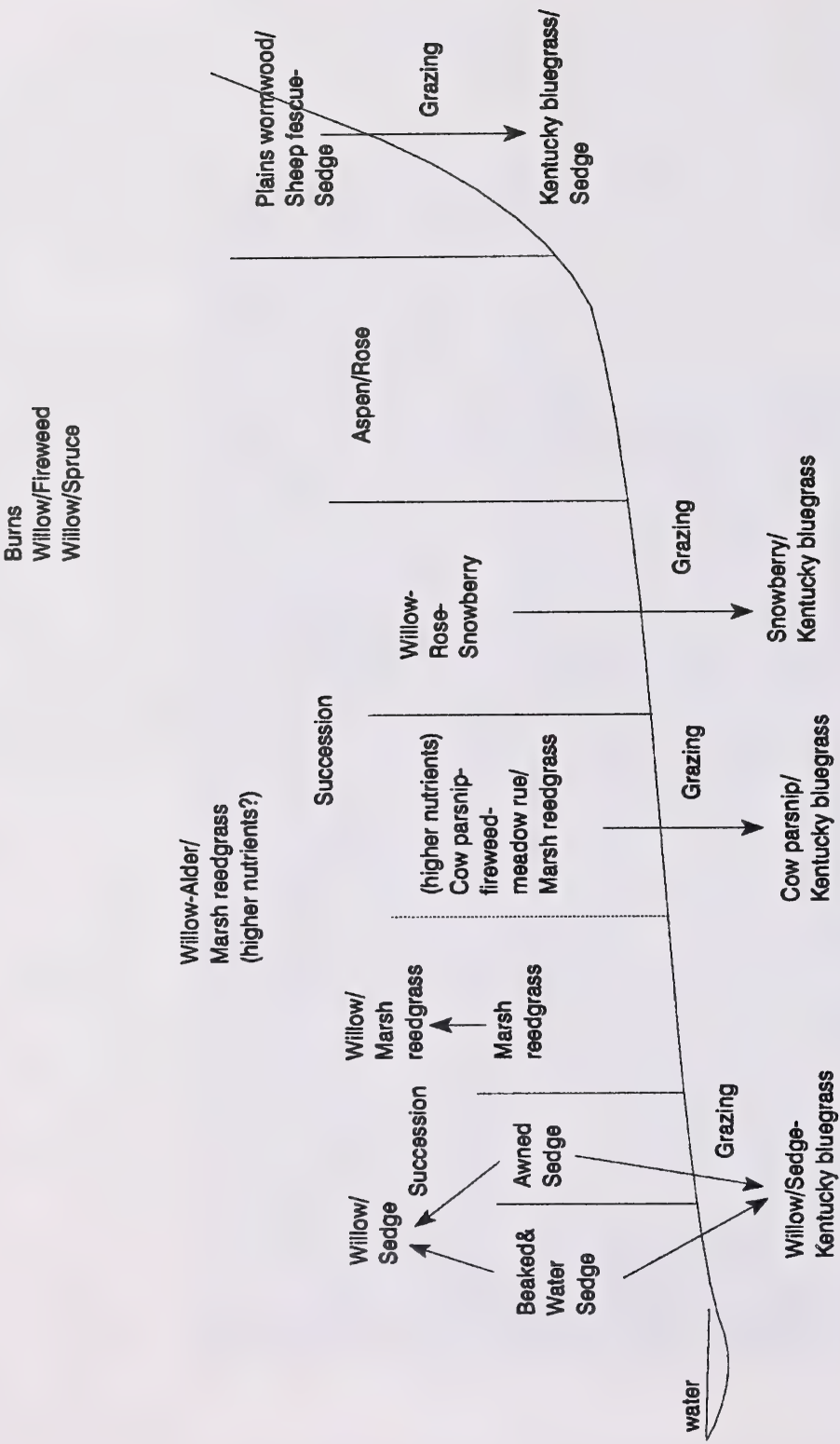

Figure 7. Overview of native shrub and grassland communities in the Central Mixedwood subregion. 


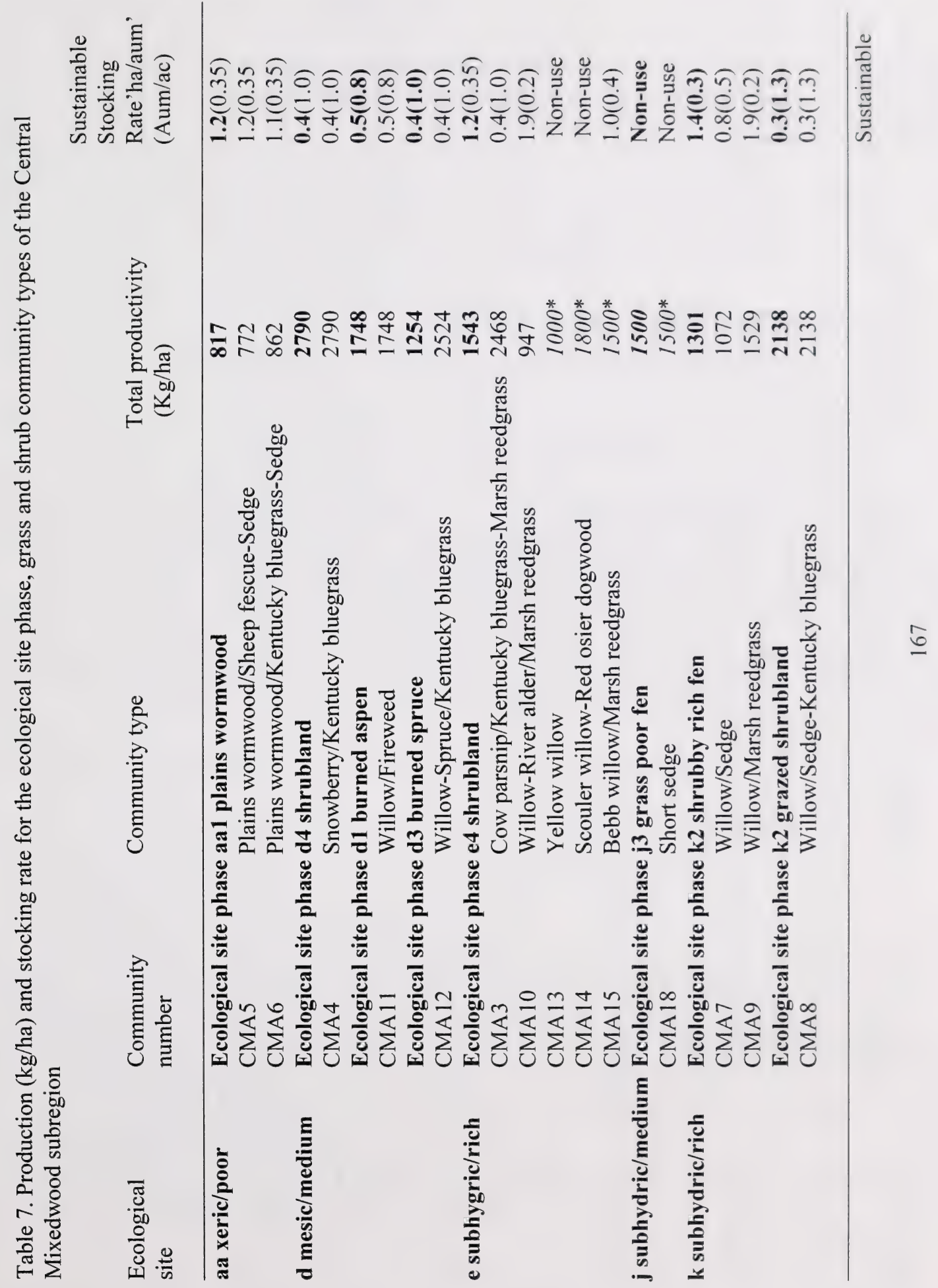



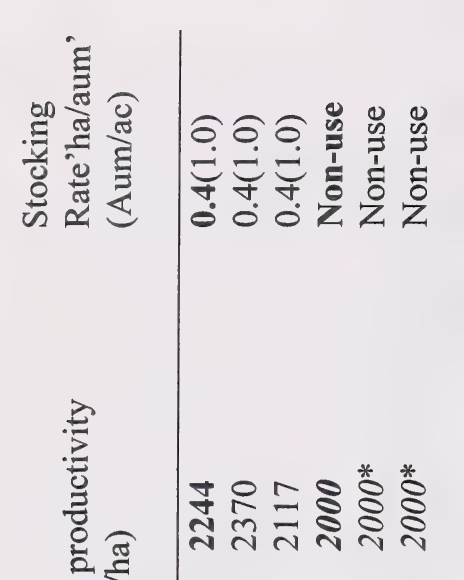

퓽 비

$\underset{0}{\infty}$ 


\section{Central Mixedwood Grass and Shrublands}

1. Shrubland dominated by willow, bog birch, alder, understory spruce ................................ Grass-dominated, or if shrub-dominated, upland species like snowberry ............................ 7

\section{SHRUBLANDS}

2. Sedge, marsh reedgrass dominated understory, wet sites or riparian or seepage areas dominated by yellow, Scouler or Bebb willow...................................................................3a Communities of fire origin, willow, alder, fireweed, understory spruce dominated ............5

3. Ungrazed, sedge and marsh reedgrass dominated understory .......................................... Grazed community type with Kentucky bluegrass Willow/Sedge-Kentucky Bluegrass (CMA8)

3a. Riparian areas dominated by yellow willow........... Yellow willow(CMA13)

Seepage areas dominated by Bebb or Scouler's willow or edges of lakes and sloughs dominated by Marsh reedgrass or sedge in understory...................................................... $3 \mathrm{~b}$

3b. Upland seepage areas dominated by Bebb or Scouler's willow.......................................4a Wet lowland sites dominated by Marsh reedgrass or sedge species....................................

4. Wetland sedges dominate understory..... Willow/Sedge (CMA7) Marsh reedgrass dominates understory. Willow/Marsh Reedgrass (CMA9)

4a. Bebb willow dominated community..... Bebb willow/Marsh reedgrass(CMA15) Scouler's willow dominated community......Scouler willow-Red osier dogwood(CMA16)

5. Willow, alder dominated community Willow-Alder/Marsh Reedgrass (CMA10) Willow, fireweed and understory spruce dominated communities.

6. Willow, fireweed dominated. Willow/Fireweed (CMA11) Willow, spruce dominated Willow-Spruce/Kentucky Bluegrass (CMA12)

\section{GRASSLANDS}

7. Lowland sites dominated by sedge, marsh reedgrass, swamp horsetail or tall manna grass.

Upland sites dominated by snowberry, sage, or cow parsnip.

7a. Boggy areas dominated by short sedge.

Short sedge (CMA18)

Freshwater areas dominated by Marsh reedgrass, sedge, swamp horsetail or tall manna grass.

7b. Area dominated by sedge or Marsh reedgrass. 8 Area dominated by tall manna grass or swamp horsetail. $7 \mathrm{c}$

7c. Swamp horsetail dominated site, very wet. Swamp horsetail (CMA16) Tall manna grass dominated site. Tall manna grass (CMA17)

8. Wet sites dominated by wetland sedge Sedge Meadow (CMA1) Slightly drier sites dominated by marsh reedgrass Marsh Reedgrass Meadow (CMA2)

9. Moist, nutrient rich seepage areas or snowberry dominated areas adjacent to rivers. Dry, sandy sites or south facing slopes dominated by sage or grasses and upland sedge.....11

10. Moist nutrient rich seepage areas dominated by cow parsnip................... Cow Parsnip/Kentucky Bluegrass-Marsh Reedgrass (CMA3) Well drained, gravelly sites adjacent to rivers and 
dominated by snowberry...................................Snowberry/Kentucky Bluegrass (CMA4)

11. Dry, sandy south facing slopes dominated by plains wormwood, sheep fescue, and

\section{(CMA5)}

$$
\text { sedge............................................Plains Wormwood/Sheep Fescue-Sedge }
$$

Grazed, sandy grasslands dominated by Kentucky

bluegrass..

..Plains Wormwood/Kentucky Bluegrass-Sedge (CMA6) 


\section{CMA1. Sedge meadows \\ (Carex aquatilis, $C$. rostrata, $C$. atherodes)}

$\mathbf{n = 5}$ This wetland community type is found near fresh water. The sedge meadow is a poorly drained community. As one moves to the drier edges marsh reedgrass becomes predominant. Willows will invade into both the sedge and marsh reedgrass dominated meadows. The sedge meadow community is very productive, but the high water table, particulary in the spring when the sedge species are most palatable, restricts livestock movement. One study done in the Yukon found that crude protein on these meadows declined from a high of $10 \%$ in May to less than $5 \%$ in September (Bailey et al. 1992).

Beaked sedge found in abundance in this community is usually associated with nitrogen rich conditions and moving water (Brierly et al. 1985). Water sedge is often found in abundance in this community type and is associated with calcium rich stagnant water (MacKinnon et al. 1992).

\section{Plant COMPOSITION CANOPY COVER(\%)}

\section{FORBS}

MEAN RANGE CONST.

MARSH SKULLCAP

(Scutellaria galericulata) $5 \quad 0-25 \quad 20$

NODDING BEGGAR TICKS

(Bidens cernua) $\quad 3 \quad 0-13 \quad 20$

DANDELION

(Taraxacum officinale) $\quad 1 \quad 0-3 \quad 20$

\section{GRASSES}

BEAKED SEDGE

(Carex rostrata)

AWNED SEDGE

(Carex atherodes)

WATER SEDGE

(Carex aquatilis)

MARSH REEDGRASS

(Calamagrostis canadensis)8

$48 \quad 8-73 \quad 100$

$13 \quad 0-57 \quad 40$

0-7 $\quad 100$

$0-18 \quad 60$

\section{ENVIRONMENTAL VARIABLES}

MOISTURE REGIME (MEAN):

SUBHYDRIC-HYGRIC

NUTRIENT REGIME (MEAN

$\mathrm{RiCH}$

ELEVATION:

$485(150-606) \mathrm{M}$

SOIL DRAINAGE (MEAN):

POORLY TO VERY POORLY

RANGELAND HEALTH RATING:

HEALTHY

\section{FORAGE PRODUCTION(KG/HA)}

GRASS 2209(1498-300)

FORB 161(0-644)

TOTAL 2370(1498-3000)
ECOLOGICALLY SUSTAINABLE STOCKING RATE $0.4 \mathrm{HA} / \mathrm{AUM}$ (1.0 AUM/AC) (AUTUMN ONLY) 


\section{CMA2. Marsh reedgrass meadow \\ (Calamagrostis canadensis)}

$\mathbf{n}=6$ This community is found on the edges of sedge meadows and moist draws where the water table is lower. The lower water table makes this community accessible for most of the grazing season. Willow will invade onto these sites to form the Willow/Marsh reedgrass community type. Increased grazing pressure on these sites will cause marsh reedgrass to decline and their will be an invasion of Kentucky bluegrass and dandelion. These sites are highly productive for domestic livestock and should be rated as primary range.

\section{Plant COMPOSITION CANOPY COVER(\%)} MEAN RANGE CONST.

\section{TREES}

WHITE BIRCH

(Betula papyrifera)

$2 \quad 0-14$

17

SHRUBS

WILLOW SPP.

(Salix spp.)

\section{FORBS}

NODDING BEGGARTICKS

(Bidens cernua)

LEAFY-BRACTED ASTER

(Aster sibricus)

DOCK , SORREL

(Rumex crispus)

GRASSES

MARSH REEDGRASS

(Calamagrostis canadensis) 56

BEAKED SEDGE

(Carex rostrata)

WATER SEDGE

(Carex aquatilis)

AWNED SEDGE

(Carex atherodes)

$1 \quad 0-2 \quad 33$

$1 \quad 0-1 \quad 17$

$\begin{array}{lll}\text { T } & 0-1 \quad 17\end{array}$

$1 \quad 0-1 \quad 33$

34-83 100

$0-28 \quad 17$

$0-14 \quad 33$

$11 \quad 0-33 \quad 67$

\section{ENVIRONMENTAL VARIABLES}

MOISTURE REGIME (MEAN): HYGRIC

NUTRIENT REGIME (MEAN): MEDIUM TO RICH

ELEVATION:

$320(150-758) \mathrm{M}$

SOIL DRAINAGE (MEAN):

POORLY

RANGELAND HEALTH RATING:

HEALTHY

FORAGE PRODUCTION(KG/HA)

GRASS 2068(1052-5110)

FORB 6(0-18)

SHRUB 42(0-254)

TOTAL 2117(1070-5110)

ECOLOGICALLY SUSTAINABLE STOCKING RATE $0.4 \mathrm{HA} / \mathrm{AUM}(1.0 \mathrm{AUM} / \mathrm{AC})$ 


\section{CMA3. Cow parsnip/Kentucky bluegrass-Marsh reedgrass \\ (Heracleum lanatum/Poa pratensis-Calamagrostis canadensis)}

$\mathbf{n = 1}$ This community type is found on fine textured, silty soils adjacent to the Willow river near Wabasca. It represents a Willow/Cow parsnip/Marsh reedgrass community that has been cleared and then grazed extensively. The heavy grazing pressure has allowed dandelion and Kentucky bluegrass to invade onto the site. The high nutrient and moisture regime of this community type makes it extremely productive. Once cleared of shrubs it can provide a significant amount of forage for domestic livestock.

\section{PLANT COMPOSITION CANOPY COVER(\%)} MEAN RANGE CONST.

\section{SHRUBS}

GREEN ALDER

(Alnus crispa)

PRICKLY ROSE

(Rosa acicularis)

FORBS

COW PARSNIP

(Heracleum lanatum)

HORSETAIL

(Equisetum arvense) 33

DANDELION

(Taraxacum officinale)

FIREWEED

(Epilobium angustifolium) 19

YELLOW PEAVINE

(Lathyrus ochroleucus)

GRASSES

KENTUCKY BLUEGRASS

(Poa pratensis)

MARSH REEDGRASS

(Calamagrostis canadensis) 10

FRINGED BROME

(Bromus ciliatus) 2

\section{ENVIRONMENTAL VARIABLES}

MOISTURE REGIME (MEAN): SUBHYGRIC

NUTRIENT REGIME (MEAN): $\mathrm{RICH}$

ELEVATION:

$606 \mathrm{M}$

SOIL DRAINAGE (MEAN): MODERATELY WELL

RANGELAND HEALTH RATING: HEALTHY WITH PROBLEMS

FORAGE PRODUCTION(KG/HA)

GRASS 200

FORB 1798

SHRUB 470

TOTAL 2468

ECOLOGICALLY SUSTAINABLE STOCKING RATE
$0.4 \mathrm{HA} / \mathrm{AUM}(1.0 \mathrm{AUM} / \mathrm{AC})$

100 


\section{CMA4. Snowberry/Kentucky bluegrass (Symphoricarpos occidentalis/Poa pratensis)}

$\mathbf{n = 4}$ This snowberry dominated community type appears to be common on level, well drained, gravelly areas along rivers throughout Northern Alberta. In the absence of disturbance this community type appears to be dominated by snowberry, rose, fireweed, slender wheatgrass and marsh reedgrass. Heavy grazing pressure causes the native forbs and grasses to decline and allows Kentucky bluegrass, dandelion and clover to increase. Because these clearings are some of the only natural openings throughout the Central Mixedwood they tend to be heavily utilized by livestock. Snowberry which is unpalatable to livestock will remain even under extreme grazing pressure.

\section{Plant COMPOSITION CANOPY COVER(\%)} MEAN RANGE CONST.

\section{SHRUBS}

PRICKLY ROSE

(Rosa acicularis)

BUCKBRUSH

(Symphoricarpos

occidentalis)

WILLOW

(Salix spp.)

FORBS

STRAWBERRY

(Fragaria virginiana) 1

ClOVER

(Trifolium repens)

DANDELION

(Taraxacum officinale)

YARROW

(Achllea millefolium)

(Vicia americana)

GRASSES

MARSH REEDGRASS

(Calamagrostis canadensis)7

SLENDER WHEATGRASS

(Agropyron trachycaulum)7

KENTUCKY BLUEGRASS

(Poa pratensis)

PRAIRIE SEDGE

(Carex prairea)

$3 \quad 0-9 \quad 50$

$19 \quad 1-30 \quad 100$

$5 \quad 0-8 \quad 75$

$1 \quad 0-1 \quad 75$

$29 \quad 0-54 \quad 75$

$32 \quad 5-49 \quad 100$

$2 \quad 1-4 \quad 100$

$1 \quad 0-1 \quad 50$

$0-24 \quad 50$

3-13 100

$38 \quad 16-73 \quad 100$

$1 \quad 0-1 \quad 25$

\section{ENVIRONMENTAL VARIABLES}

MOISTURE REGIME (MEAN):

MESIC

NUTRIENT REGIME (MEAN):

MEDIUM TO RICH

ELEVATION:

$$
\text { 576-606(586) M }
$$

SOIL DRAINAGE (MEAN):

WELL

RANGELAND HEALTH RATING:

HEALTHY WITH PROBLEMS

\section{FORAGE PRODUCTION(KG/HA)}

GRASS $1337(800-1800)$

FORB 1311(200-2390)

SHRUB 141(0-424)

TOTAL 2790(2000-3614)

ECOLOGICALLY SUSTAINABLE STOCKING RATE

$0.4 \mathrm{HA} / \mathrm{AUM}(1.0 \mathrm{AUM} / \mathrm{AC})$ 


\section{CMA5. Plains wormwood/Sheep fescue-Sedge \\ (Artemisia campestris/Festuca saximontana-Carex spp.)}

$\mathbf{n = 3} \quad$ This community type is found on coarse textured, sandy soils. It is generally found on hilltops and southfacing slopes in openings among Jack pine on the uplands and black spruce in the lowlands. This community type was also described on similar site conditions in the Dry Mixedwood subregion. This community would be considered either secondary or non-use range for domestic livestock because of the low forage production and fragile nature of the community.

\section{Plant COMPOSITION CANOPY COVER(\%)} MEAN RANGE CONST.

\section{SHRUBS}

\section{SASKATOON}

(Amelanchier

BLUEBERRY

(Vaccinium myrtilloides) $3 \quad 0-8 \quad 33$

\section{FORBS}

SMOOTH SCOURING RUSH

(Equisetum laevigatum)

PLAINS WORMWOOD

(Artemisia campestris) $\quad 9 \quad 2-13 \quad 100$

LOW GOLDENROD

(Solidago missouriensis) $2 \quad 1-3 \quad 66$

BEARBERRY

(Arctostaphylos uva-ursi) $5 \quad 0-8 \quad 67$

GRASSES

KENTUCKY BLUEGRASS

(Poa pratensis)

(Oryzopsis pungens)

SLENDER WHEATGRASS

(Agropyron trachycaulum $) 2$

SEDGE

(Carex spp)

SHEEP FESCUE

(Festuca saximontana)

$3 \quad 1-4 \quad 100$

$0-12 \quad 67$

$1-5 \quad 100$

$7-10 \quad 100$

$7-10 \quad 100$

\section{ENVIRONMENTAL VARIABLES}

MOISTURE REGIME (MEAN):

SUBMESIC-SUBXERIC

NUTRIENT REGIME (MEAN):

$$
\text { MEDIUM }
$$

ELEVATION:

$$
611(576-652) \mathrm{M}
$$

SOIL DRAINAGE (MEAN):

$$
\text { RAPIDLY }
$$

SLOPE(RANGE):

ASPECT:

$$
22(15-30) \%
$$

SOUTH TO WESTERLY

RANGELAND HEALTH RATING:

HEALTHY

FORAGE PRODUCTION(KG/HA)

GRASS $469(270-612)$

FORB 303(200-452)

TOTAL 772(470-978)

ECOLOGICALLY SUSTAINABLE STOCKING RATE $1.2 \mathrm{HA} / \mathrm{AUM}(0.35$ AUM/AC $)$ 


\section{CMA6. Plains wormwood/Kentucky bluegrass-Sedge \\ (Artemisia campestris/Poa pratensis-Carex spp.)}

$\mathbf{n}=\mathbf{1}$ This community type is similar to the Plains wormwood/Sheep fescue-Sedge community type, but heavy grazing pressure and a higher nutrient and moisture regime has allowed Kentucky bluegrass to invade onto the site.

\section{PlaAT COMPOSITION CANOPY COVER(\%) MEAN RANGE CONST.}

\section{SHRUBS}

SASKATOON

(Amelanchier alnifolia) $2 \quad$ - $\quad 100$

CHOKECHERRY

$\begin{array}{llll}\text { (Prunus virginiana) } & 8 & - & 100\end{array}$

SNOWBERRY

(Symphoricarpos

occidentalis)

FORBS

MEADOW PARSNIP

(Zizia aptera)

PLAINS WORMWOOD

(Artemisia campestris) $4 \quad$ - $\quad 100$

LOW GOLDENROD

(Solidago missouriensis) $2 \quad$ - $\quad 100$

BEARBERRY

(Arctostaphylos uva-ursi) $10 \quad$ - 100

GRASSES

KENTUCKY BLUEGRASS

$\begin{array}{lll}\text { (Poa pratensis) } & 49 & -\end{array}$

NORTHERN RICEGRASS

$\begin{array}{llll}\text { (Oryzopsis pungens) } & 4 & - & 100\end{array}$

SLENDER WHEATGRASS

(Agropyron trachycaulum)3 $\quad$ - $\quad 100$

SEDGE

(Carex spp)

SHEEP FESCUE

$\begin{array}{lll}\text { (Festuca saximontana) } & 1 & -\end{array}$

\section{ENVIRONMENTAL VARIABLES}

MOISTURE REGIME (MEAN):

SUBMESIC

NUTRIENT REGIME (MEAN):

MEDIUM

ELEVATION:

$606 \mathrm{M}$

SOIL DRAINAGE (MEAN):

$$
\text { RAPIDLY }
$$

SLOPE(RANGE):

$15 \%$

ASPECT:

SOUTH TO WESTERLY

RANGELAND HEALTH RATING:

HEALTHY WITH PROBLEMS

FORAGE PRODUCTION(KG/HA)

GRASS 824

FORB 38

TOTAL 862

ECOLOGICALLY SUSTAINABLE STOCKING RATE

$1.1 \mathrm{HA} / \mathrm{AUM}(0.35 \mathrm{AUM} / \mathrm{AC})$ 


\section{CMA7. Willow/Sedge \\ (Salix spp./Carex spp.)}

$\mathbf{n}=\mathbf{6}$ This community type is found along the edges of sedge meadows and in moist depressions. Willow becomes established at the edges of the sedge meadows due to the shorter duration of standing water. Increased flooding and prolonged waterlogging may result in the disappearance of willow and a transition to a water sedge meadow.

These sites are fairly productive but difficult to graze due to the moist ground conditions and heavy shrub cover which reduces access and mobility within the area.

\section{Plant Composition CanopyCover(\%)}

\section{SHRUBS}

MEAN RANGE CONST.

WILLOW SPP.

(Salix spp.)

$57 \quad 26-85 \quad 100$

FORBS

MINT

(Mentha arvensis)

GREEN SOREL

(Rumex acetosa)

FIREWEED

(Epilobium angustifolium) 3

HORSETAIL

(Equisetum arvense) $\quad 10 \quad 0-60 \quad 34$

GRASSES

AWNED SEDGE

(Carex atherodes)

$12 \quad 0-31 \quad 50$

MARSH REEDGRASS

(Calamagrostis canadensis) $12 \quad 0-20 \quad 83$

BEAKED SEDGE

(Carex rostrata)

WATER SEDGE

(Carex aquatilis)

$7 \quad 0-20 \quad 50$

$14 \quad 0-43 \quad 50$

\section{ENVIRONMENTAL VARIABLES}

MOISTURE REGIME (MEAN):

SUBHYDRIC

NUTRIENT REGIME (MEAN):

$\mathrm{RICH}$

ELEVATION:

393(150-636) M

SOIL DRAINAGE (MEAN):

POORLY

RANGELAND HEALTH RATING:

HEALTHY

FORAGE PRODUCTION(KG/HA)

GRASS 852(0-1734)

FORB 96(70-150)

SHRUB $\quad 100(0-364)$

TOTAL 1072(214-2218) 


\section{CMA8. Willow/Sedge-Kentucky bluegrass \\ (Salix spp./Carex spp.-Poa pratensis)}

$\mathbf{n = 4}$ This community type is very similar to the Willow/Sedge community, but has been heavily grazed favouring the growth of Kentucky bluegrass and dandelion. Continued heavy grazing pressure will eventually lead to a community that is similar to the Kentucky bluegrass/Dandelion dominated community type.

\section{PLANT COMPOSITION CANOPY COVER(\%)}

MEAN RANGE CONST.

\section{SHRUBS}

WILLOW SPP.

(Salix spp.)

PRICKLY ROSE

(Rosa acicularis)

25

$1-40$

100

$3 \quad 0-10 \quad 25$

FORBS

STRAWBERRY

(Fragaria virginiana) $3 \quad 0-11 \quad 25$

DANDELION

(Taraxacum offincinale) 5

MINT

(Mentha arvensis)

ClOVER

$\begin{array}{lr}\text { (Trifolium spp.) } & 9 \\ \text { ARROW LEAVED COLTSFOOT }\end{array}$

(Petasites sagittatus) 9

GRASSES

SEDGE

(Carex rostrata, aquatilis

atherodes.)

KENTUCKY BLUEGRASS

(Poa pratensis)

$3 \quad 0-6 \quad 75$

$0-44 \quad 25$

$0-15 \quad 50$

$40 \quad 12-61 \quad 100$

$21 \quad 7-42 \quad 100$

\section{ENVIRONMENTAL VARIABLES}

MOISTURE REGIME (MEAN): SUBHYGRIC

NUTRIENT REGIME (MEAN): $\mathrm{RICH}$

ELEVATION:

$576 \mathrm{M}$

SOIL DRAINAGE (MEAN): IMPERFECTLY

RANGELAND HEALTH RATING: HEALTHYWITH PROBLEMS

\section{FORAGE PRODUCTION(KG/HA)}

GRASS 2121(1566-2478)

FORB 547(492-1204)

TOTAL 2138(2770-2970)

ECOLOGICALLY SUSTAINABLE STOCKING RATE $0.3 \mathrm{HA} / \mathrm{AUM}$ (1.3 AUM/AC) 


\section{CMA9. Willow/Marsh reedgrass \\ (Salix spp./Calamagrostis canadensis, C. inexpansa)}

$\mathbf{n}=9 \quad$ The Marsh reedgrass community type is found along the edges of sedge meadows and in moist depressions Willow will invade onto these sites to form the Willow/Marsh reedgrass community type. Increased grazing pressure on these sites will cause marsh reedgrass to decline and there will be an invasion of Kentucky bluegrass and dandelion. These sites are highly productive for domestic livestock and should be rated as primary range. Increased flooding and prolonged waterlogging may result in the disappearance of willow and a transition to a water sedge meadow.

These sites are fairly productive but difficult to graze due to the moist ground conditions and heavy shrub cover which reduces access and mobility within the area.

\section{Plant COMPOSITION CANOPY COVER(\%)} MEAN RANGE CONST.

\section{SHRUBS}

WILLOW SPP.

(Salix spp.)

FLAT LEAVED WILLOW

(Salix planifolia)

BEBB WILLOW

(Salix bebbiana)

FORBS

\section{MINT}

(Mentha arvensis)

DANDELION

(Taraxacum officinale)

$\begin{array}{lll}44 & 0-80 & 80 \\ 12 & 0-60 & 22 \\ 2 & 0-20 & 11\end{array}$

$0-20$

$1 \quad 0-7 \quad 44$

$2 \quad 0-12 \quad 56$
GRASSES

KENTUCKY BLUEGRASS

(Poa pratensis)

$2 \quad 0-7 \quad 33$

MARSH REEDGRASS

$\begin{array}{lll}\text { (Calamagrostis canadensis) } 22 & 0-47 & 89 \\ \text { BEAKED SEDGE } & \end{array}$

(Carex rostrata) $\quad 5 \quad 0-22 \quad 56$

WATER SEDGE

(Carex aquatilis) $\quad 6 \quad 0-23 \quad 33$

NORTHERN REEDGRASS

(Calamagrostis inexpansa) 6

\section{ENVIRONMENTAL VARIABLES}

MOISTURE REGIME (MEAN):

SUBHYGRIC

NUTRIENT REGIME (MEAN):

$\mathrm{RICH}$

ELEVATION:

333-576(537) M

SOIL DRAINAGE (MEAN):

POORLY

RANGELAND HEALTH RATING:

HEALTHY

FORAGE PRODUCTION(KG/HA)

$\begin{array}{ll}\text { GRASS } & 1050(318-2010) \\ \text { FORB } & 107(0-270) \\ \text { SHRUB } & 208(0-554) \\ \text { TOTAL } & 1529(588-2118)\end{array}$

ECOLOGICALLY SUSTAINABLE STOCKING RATE $0.6 \mathrm{HA} / \mathrm{AUM}$ (0.65 AUM/AC) 


\section{CMA10. Willow-River alder/Marsh reedgrass \\ (Salix spp-Alnus tenuifolia/Calamagrostis canadensis)}

$\mathbf{n}=6$ This community type represents a tall willow and alder dominated type that is usually represented as an AIA aspen stand on phase III maps. It is typically found in very moist, poorly drained areas. Black spruce communities are usually found associated with this community type on the wetter edges. The understory of this community type is fairly open allowing for easy access by livestock. When this community is situated next to trails or seismic lines it is moderately utilized by livestock.

PLANT COMPOSITION CANOPY COVER(\%) MEAN RANGE CONST.

\section{SHRUBS}

FLAT LEAVED WILLOW

(Salix planifolia)
WILLOW SPP.
(Salix spp.)

RIVER ALDER

(Alnus tenuifolia)

GREEN ALDER

(Alnus crispa)

WILD RED RASPBERRY

(Rubus idaeus) $\quad 11 \quad 0-33 \quad 50$

BRACTED HONEYSUCKLE

$\begin{array}{llll}\text { (Lonicera involucrata) } & 4 & 0-13 \quad 50\end{array}$

FORBS

STRAWBERRY

(Fragaria virginiana) 1

SWEET SCENTED BEDSTRAW

(Galium triflorum) 3

WILD SARSAPARILLA

(Aralia nudicaulis) 4

DEWBERRY

(Rubus pubscens)

3

GRASSES

MARSH REEDGRASS

(Calamagrostis canadensis)40 $\quad 14-60 \quad 100$

BEAKED SEDGE

(Carex rostrata)
$6 \quad 0-30 \quad 33$

$32 \quad 0-65 \quad 67$

$20 \quad 0-40 \quad 67$

$9 \quad 0-35 \quad 33$

$0-3 \quad 33$

$0-11 \quad 67$

$0-13 \quad 33$

$0-11 \quad 50$

$0-27 \quad 17$

\section{ENVIRONMENTAL VARIABLES}

MOISTURE REGIME (MEAN): SUBHYGRIC-HYGRIC

NUTRIENT REGIME (MEAN): $\mathrm{RICH}$

ELEVATION:

$576 \mathrm{M}$

SOIL DRAINAGE (MEAN): IMPERFECTLY

RANGELAND HEALTH RATING: HEALTHY

FORAGE PRODUCTION(KG/HA)

GRASS 702(118-1102)

FORB $\quad 184(18-470)$

SHRUB 61(0-132)

TOTAL 947(592-1296)

ECOLOGICALLY SUSTAINABLE STOCKING RATE $1.9 \mathrm{HA} / \mathrm{AUM}(0.2 \mathrm{AUM} / \mathrm{AC})$ 


\section{CMA11. Willow/Fireweed \\ (Salix spp./Epilobium angustifolium)}

$\mathbf{n}=\mathbf{1} \quad$ This community type represents a 3 year old burn of a white spruce forest. Fireweed and marsh reedgrass early successional species quickly dominate the community after a fire. As this community undergoes succession the herbaceous understory will be suppressed as a result of shading by white spruce. Eliminating the tree canopy cover has increased the forage production of this site from $50-100 \mathrm{~kg} / \mathrm{ha}$ under a spruce moss forest to over 1700 $\mathrm{kg} / \mathrm{ha}$ on this community type.

Plant COMPOSITION CANOPY COVER(\%) MEAN RANGE CONST.

\section{TREeS}

ASPEN

(Populus tremuloides)

WHITE SPRUCE

(Picea glauca)

SHRUBS

WILLOW SPP.

(Salix spp.)

FORBS

STRAWBERRY

$\begin{array}{llll}\text { (Fragaria virginiana) } \quad 2 \quad & - & 100\end{array}$

FIREWEED

(Epilobium angustifolium)37 - $\quad 100$

YARROW

(Achillea millefolium) 2

LARGE LEAVED YELLOW AVENS

(Geum macrophyllum)

\section{GRASSES}

MARSH REEDGRASS

(Calamagrostis canadensis)19 - $\quad 100$

HAIR-LIKE SEDGE

(Carex capillaris)

\section{ENVIRONMENTAL VARIABLES}

MOISTURE REGIME (MEAN):

SUBHYGRIC-MESIC

NUTRIENT REGIME (MEAN):

MEDIUM

ELEVATION:

$150 \mathrm{M}$

SOIL DRAINAGE (MEAN):

MODERATELY WELL

RANGELAND HEALTH RATING:

HEALTHY

FORAGE PRODUCTION(KG/HA)

GRASS $\quad 190$

FORB $\quad 1322$

SHRUB 236

TOTAL 1748

ECOLOGICALLY SUSTAINABLE STOCKING RATE $0.5 \mathrm{HA} / \mathrm{AUM}(0.8 \mathrm{AUM} / \mathrm{AC})$ 


\section{CMA12. Willow-Spruce/Kentucky bluegrass \\ (Salix spp.-Picea glauca/Poa pratensis)}

n=1 This community represents an old spruce community which burned in 1968, succeeded to willow, and is now succeeding back to white spruce. After the fire, the canopy was opened up allowing for good forage productivity. Consequently, cattle grazing was quite heavy allowing Kentucky bluegrass and clover to establish. Thistle is now beginning to invade and will expand to other areas if not controlled. As the spruce continues to mature, the increasing canopy cover will cause a decline in overall production and this site will eventually become non-use for domestic livestock.

\section{PLANT COMPOSITION CANOPY COVER(\%) \\ MEAN RANGE CONST.}

TREES

LARCH

(Larix laricina)

WHITE SPRUCE(UNDERSTORY)

(Picea glauca)

SHRUBS

WILLOW SPP.

(Salix spp.)

FORBS

ClOVER

(Trifolium sp.)

DANDELION

(Taraxacum officinale)

MARSH HEDGE NETTLE

(Stachys palustris)

BISHOP'S CAP

(Mitella nuda)

CANADA THISTLE

(Cirsium arvense)

GRASSES

KENTUCKY BLUEGRASS

(Poa pratensis)

\section{ENVIRONMENTAL VARIABLES}

\author{
MOISTURE REGIME (MEAN): \\ SUBHYGRIC \\ NUTRIENT REGIME (MEAN): \\ $\mathrm{RICH}$ \\ ELEVATION: \\ $667 \mathrm{M}$ \\ SOIL DRAINAGE (MEAN): \\ MODERATELY WELL TO IMPERFECTLY \\ RANGELAND HEALTH RATING: \\ UNHEALTHY
}

FORAGE PRODUCTION(KG/HA)

$\begin{array}{ll}\text { GRASS } & 1985 \\ \text { FORB } & 540 \\ \text { SHRUB } & 0 \\ \text { TOTAL } & 2524\end{array}$

ECOLOGICALLY SUSTAINABLE STOCKING RATE

$0.4 \mathrm{HA} / \mathrm{AUM}(1.0 \mathrm{AUM} / \mathrm{AC})$ 


\section{CMA13. Yellow willow}

(Salix lutea)

$\mathbf{n}=\mathbf{1}$ This community type occurs on moist alluvial deposits which are adjacent to streams and rivers. This community can persist for some time if the site is subject to frequent flooding. However in the absence of disturbance it will eventually undergo succession to a spruce dominated community type. Thompson and Hansen (2002) described this community in the grassland natural region of Southern Alberta. They found that this community type disappeared as one moved north into the Parkland and it was replaced by basket willow and flat leaved willow dominated community types. Typically there is little understory vegetation found in this community type and it should be rated as non-use for livestock.

PLANT COMPOSITION CANOPY COVER (\%) MEAN RANGE CONST.

\section{SHRUBS}

YELLOW WILLOW

(Salix lutea)

(Salix lucida)

RIVER ALDER

(Alnus tenuifolia)

\section{FORBS}

HORSETAIL

(Equisetum arvense)

VEINY MEADOW RUE

(Thalictrum venulosum)

DANDELION

(Taraxaxum officinale)
SHINING WILLOW

\section{GRAMINOIDS}

MARSH REEDGRASS

(Calamagrostis canadensis) 10

KENTUCKY BLUEGRASS

(Poa pratensis)

QUACKGRASS

(Agropyron repens)
100

100

100

\section{0}

10

3

100

100

100

\section{ENVIRONMENTAL VARIABLES}

MOISTURE REGIME: HYGRIC

NUTRIENT REGIME: RICH

Elevation: $600 \mathrm{M}$

SOIL DRAINAGE: IMPERFECTLY

RANGELAND HEALTH RATING:

HEALTHY

Forage Production (KG/HA)

TOTAL $1000 *$ EstIMATE

ECOLOGICALLY SUSTAINABLE STOCKING RATE NON-USE 


\section{CMA14. Scouler willow-Red osier dogwood (Salix scouleriana-Cornus stolonifera)}

$\mathbf{n}=\mathbf{1} \quad$ This community type appears to be transitional between the horsetail (hygric/rich) and shrubby rich fen (subhydric/rich) ecosites described by Beckingham and Archibald (1996). It has plant species characteristic of both ecosites. This community type is also similar to the Willow-Alder/Fern community described on moist, nutrient rich seepage areas in the Lower Foothills subregion (Lane et al. 2000). This community type is very productive, but the high shrub cover and slope conditions make it difficult to graze. Consequently, this community type should be rated as secondary or non-use range.

\section{PLANT COMPOSITION CANOPY COVER(\%)}

MEAN RANGE CONST.

\section{SHRUBS}

\section{SCOULER'S WILLOW}

(Salix scouleriana)

BRACTED HONEYSUCKLE

(Lonicera involcrata)

RED OSIER DOGWOOD

(Cornus stolonifera)

LOW BUSH CRANBERRY

(Viburnum edule)

FORBS

BUNCHBERRY

(Cornus canadensis)

COMMON HORSETAIL

(Equisetum arvensis)

FIREWEED

(Epilobium angustifolium)3

DEWBERRY

(Rubus pubescens)

STRAWBERRY

(Fragaria virginiana)

GRASSES

MARSH REEDGRASS

(Calamagrostis canadensis) 10

100

\section{ENVIRONMENTAL VARIABLES}

MOISTURE REGIME (MEAN): SUBHYGRIC

NUTRIENT REGIME (MEAN): PERMESOTROPHIC

ELEVATION:

$667 \mathrm{M}$

SOIL DRAINAGE (MEAN): MODERATELY WELL

RANGELAND HEALTH RATING: HEALTHY

FORAGE PRODUCTION(KG/HA)

GRASS $\quad 580$

FORB $\quad 1272$

TOTAL 1852

ECOLOGICALLY SUSTAINABLE STOCKING RATE NON-USE 


\section{CMA15: Bebb willow/Marsh reedgrass \\ (Salix bebbiana/Calamagrostis canadensis)}

$\mathbf{n = 3}$ This community type is found along the drier edges of marsh reedgrass meadows and in moist depressions and represents the transition between the flat leaved willow and basket willow dominated shrublands and the upland forest. Bebb willow is an upland species that prefers well drained sites. This species of willow is often found in the understory of aspen and balsam poplar dominated community types. Increased flooding and prolonged water logging may result in the disappearance of Bebb willow and favour the growth of flat leaved willow. In contrast the continued drying of the site will favour the growth of balsam poplar. These sites are fairly productive but difficul to graze due to the moist ground conditions and heavy shrub cover which reduces access and mobility within the area.

\section{PLANT COMPOSITION CANOPY COVER (\%)} Mean Range Const.

TREES

BALSAM POPLAR

(Populus balsamifera) $\quad 1 \quad 0-1 \quad 33$

\section{SHRUBS}

BEBB WILLOW

(Salix bebbiana)

FLAT LEAVED WILLOW

(Salix planifolia)

RED OSIER DOGWOOD

(Cornus stolonifera)

BRACTED HONEYSUCKLE

(Lonicera involucrata)

$\begin{array}{lll}57 & 50-70 & 100 \\ 1 & 0-3 & 33 \\ 1 & 0-3 & 66 \\ 1 & 0-3 & 66\end{array}$

\section{FORBS}

HORSETAIL

(Equisetum arvense)

TALL LUNGWORT

(Mertensia paniculata) $1 \quad 0-3 \quad 100$

SMALL ENCHANTER'S NIGHTSHADE

(Circaea alpina)

SMALL BEDSTRAW

(Galium trifidum)

$13 \quad 0-40$

33

$\begin{array}{lll}7 & 0-20 \quad 33\end{array}$

\section{GRASSES}

MARSH REEDGRASS

(Calamagrostis

canadensis)

$24 \quad 3-40 \quad 100$

\section{ENVIRONMENTAL VARIABLES}

MOISTURE REGIME: SUBHYGRIC-HYGRIC

NUTRIENT REGIME: RICH

ELEVATION(MEAN): $600 \mathrm{M}$

SOIL DRAINAGE: MOD. WELL

RANGELAND HEALTH RATING:

HEALTHY

Forage Production (KG/HA)

TOTAL $1500 *$ ESTIMATE

ECOLOGICALLY SUSTAINABLE STOCKING RATE

$1.0 \mathrm{HA} / \mathrm{AUM}(0.4 \mathrm{AUM} / \mathrm{AC})$ 


\section{CMA16. Swamp horsetail (Equisetum fluviatile)}

$\mathbf{n = 1}$ This wetland community type is found near fresh water and is often associated with shallow water around lake shores or saturated wet spots in old river channels and sloughs. This community is often only found in small isolated spots or in narrow bands around the edge of lakes. As these areas dry, swamp horsetail is often replaced by sedge species. Swamp horsetail is generally unpalatable to livestock and the areas it grows in are often to wet for livestock to access. This community type should be rated as non-use.

\section{PLANT COMPOSITION CANOPY COVER(\%)}

\section{FORBS}

MEAN RANGE CONST.

SWAMP HORSETAIL

(Equisetum fluviatile)

$97 \quad-\quad 100$

GRASSES

BEAKED SEDGE

(Carex rostrata)

TALL MANNA GRASS

(Glyceria grandis)

SLOUGH GRASS

(Beckmannia syzigachne) 1

\section{ENVIRONMENTAL VARIABLES}

MOISTURE REGIME (MEAN):

SUBHYDRIC-HYGRIC

NUTRIENT REGIME (MEAN):

$\mathrm{RICH}$

ELEVATION:

$600 \mathrm{M}$

SOIL DRAINAGE (MEAN):

POORLY TO VERY POORLY

RANGELAND HEALTH RATING:

HEALTHY

FORAGE PRODUCTION (KG/HA)

TOTAL

$2000 *$ ESTIMATE

ECOLOGICALLY SUSTAINABLE STOCKING RATE

NON-USE 


\section{CMA17. Tall manna grass \\ (Glyceria grandis)}

$\mathbf{n}=\mathbf{1} \quad$ This wetland community type is associated with the edge of the standing water of ponds, sloughs and slow meandering streams. As one moves away from the water to the drier edges the sedge meadow communities are found. This community is often only found in small isolated spots or in narrow bands around the edge of lakes. As these areas dry, tall manna grass is often replaced by sedge species. Tall manna grass is palatable to livestock, however, the areas it grows in are often to wet for livestock to access. This community type should be rated as non-use.

\section{PLANT COMPOSITION CANOPY COVER(\%)} MEAN RANGE CONST.

\section{FORBS}

MINT

$\begin{array}{llll}\begin{array}{l}\text { (Mentha arvensis) } \\ \text { PALE PERSICARIA }\end{array} & 20 & - & 100 \\ \begin{array}{l}\text { (Polygonum lapthifolium) } \\ \text { CANADA THISTLE }\end{array} & 3 & - & 100 \\ \text { (Cirsium arvense) } & 1 & - & 100\end{array}$

\section{GRASSES}

TALL MANNA GRASS

(Glyceria grandis)

60

SLOUGH GRASS

(Beckmannia syzigachne) 30

BEBB'S SEDGE

(Carex bebbii)

CREEPING SPIKE RUSH

(Eleocharis palustris)

\section{ENVIRONMENTAL VARIABLES}

MOISTURE REGIME (MEAN):

SUBHYDRIC-HYGRIC

NUTRIENT REGIME (MEAN):

$\mathrm{RICH}$

ELEVATION:

$606 \mathrm{M}$

SOIL DRAINAGE (MEAN):

VERY POORLY

RANGELAND HEALTH RATING:

HEALTHY

FORAGE PRODUCTION (KG/HA)

GRASS $\quad 2000$

TOTAL 2000*ESTIMATE

ECOLOGICALLY SUSTAINABLE STOCKING RATE

NON-USE 


\section{CMA18. Short sedge}

(Carex curta)

$\mathbf{n = 1}$ This community type was described in boggy areas adjacent to black spruce and larch dominated community types. Short sedge tends to be found in the wetter areas where there is a floating mat of peat. As these areas dry out short sedge will be replaced by willow, black spruce and larch species. Short sedge is generally unpalatable to livestock and the areas it grows in are often too wet for livestock to access. This community type should be rated as non-use.

\section{PLANT COMPOSITION CANOPY COVER(\%)}

MEAN RANGE CONST.

\section{SHRUBS}

Flat LEAVED WILLOW

$\begin{array}{llll}\text { (Salix planifolia) } & 1 & - & 100\end{array}$

FORBS

WATER HEMLOCK

(Cicuta maculata)

$1 \quad 100$

SKULL CAP

(Scutellaria galericulata) $1 \quad$ - $\quad 100$

GRASSES

SHORT SEDGE

(Carex curta)

WATER SEDGE

(Carex aquatilis)

NORTHERN REEDGRASS

(Calamagrostis inexpansa) 10

\section{ENVIRONMENTAL VARIABLES}

MOISTURE REGIME (MEAN):

SUBHYDRIC

NUTRIENT REGIME (MEAN):

MEDIUM

ELEVATION:

576-606(584) M

SOIL DRAINAGE (MEAN):

WELL

RANGELAND HEALTH RATING:

HEALTHY

FORAGE PRODUCTION (KG/HA)

TOTAL $1500 *$ ESTIMATE

ECOLOGICALLY SUSTAINABLE STOCKING RATE

NON-USE 


\section{CENTRAL MIXEDWOOD SUBREGION}

\section{TAME FORAGE COMMUNITIES}

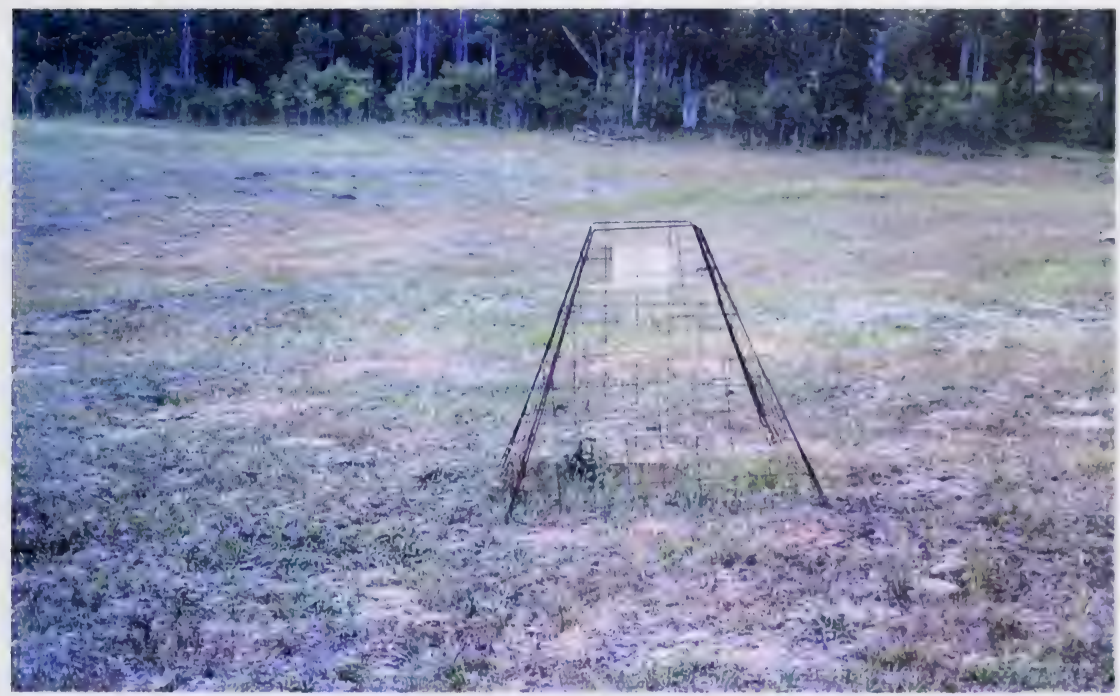

Photo 8. This range improvement clearing exhibits signs of heavy grazing pressure and is slowly being invaded by tall buttercup. 


\section{CM - TAME FORAGE COMMUNITIES \\ (Cleared areas that have been broken and seeded to tame forage)}

Throughout the Central Mixedwood subregion there are sites that have been deforested, broken, and seeded to tame forage. Usually these areas are mesic and moderately well to well drained with good nutrient levels. Because most of these tame forage stands are established on similar sites, the most influential factors affecting plant species composition are stand establishment and grazing regime.

Stand establishment is important because it determines what the initial plant species composition is going to be. Seed bed preparation and the type of seed sown are the two most important factors influencing stand establishment. Seed bed preparation is important because it helps to determine how well the sown seed germinates and establishes. If the seed bed is not well prepared the tame forage stand may establish poorly and native species can become a dominant component of the plant community.

After the stand is established, the grazing regime applied to the stand will determine the plant species composition. Generally, a light to moderate amount of grazing allows the stand to maintain itself while sustained heavy grazing causes the stand to degrade. Damage to a stand due to over grazing occurs more readily while the stand is establishing than it does when the stand is established. This is because the forage plants in an establishing stand have not had time to develop energy reserves in their roots, and are therefore, more susceptible to grazing induced damage.

Well distributed light to moderate grazing will normally maintain a forage stand similar to what was seeded on the site. These stands are generally the most productive and provide the best grazing opportunities for livestock. They are normally considered to be in good to excellent range condition. Non use or very light grazing often results in the stand becoming dominated by the forage species that is most competitive under an ungrazed situation. Plant community changes which occur under heavy grazing are dependent on the grazing history (level of use, season of use and duration of the grazing regime). Overgrazed community types develop over a long period of repeated overgrazing. If weedy species such as Tall Buttercup, become established on overgrazed sites, they can quickly become a dominant species. 


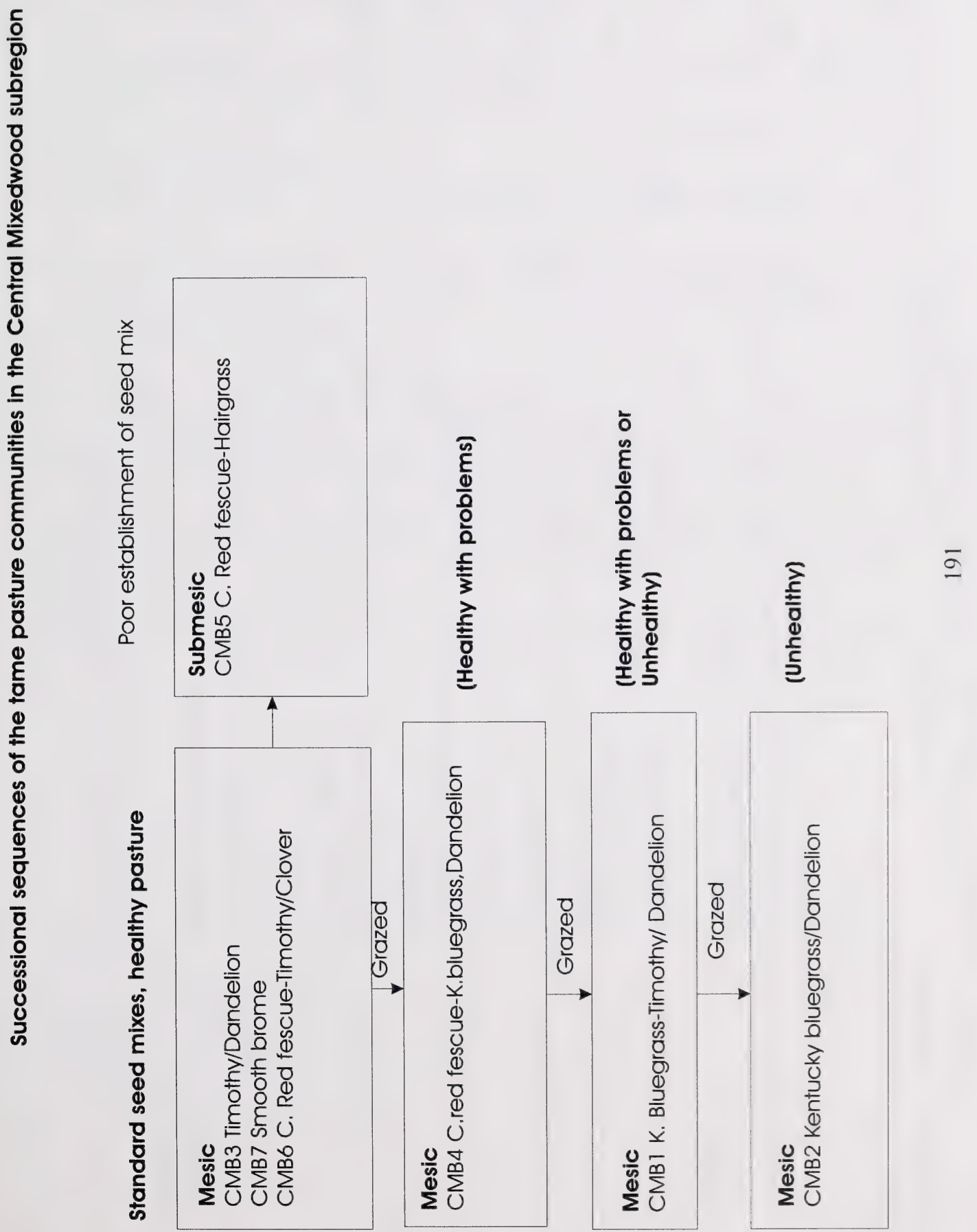




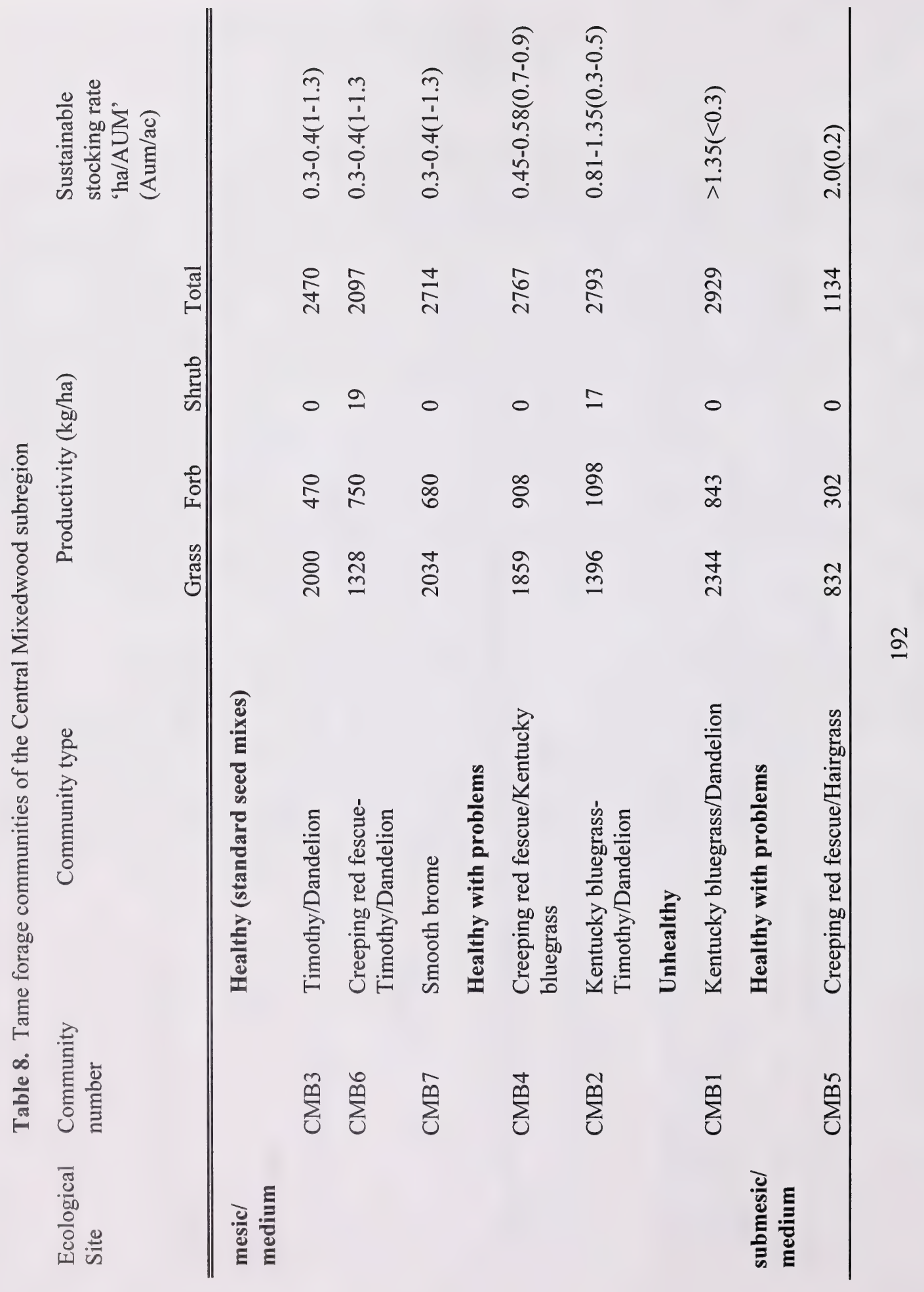




\section{Tame Grass Plant Communities - Central Mixedwood Subregion}

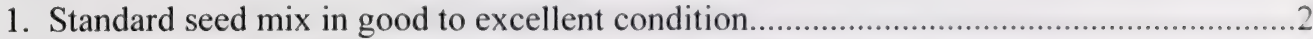
Seeded stand modified by grazing or poorly established..................................................

2. Stand dominated by smooth brome. Smooth Brome (CMB7)

Stand dominated by timothy or creeping red fescue.

3. Stand dominated by timothy Timothy/Dandelion (CMB3) Stand dominated by creeping red fescue Creeping Red Fescue-Timothy/Dandelion (CMB6)

4. Poor establishment of seed mix, extensive bare ground and many weedy species .Creeping Red Fescue-Hairgrass (CMB5) Species composition modified by grazing, mid-height grasses uncommon 5

5. Pasture moderately grazed, Kentucky bluegrass co-dominant in the stand. Creeping Red Fescue-Kentucky Bluegrass (CMB4) Pasture very heavily grazed, Kentucky bluegrass and dandelion dominate the site.

6. Pasture heavily grazed with some seeded species (brome, timothy, creeping red fescue) still present in the stand. Kentucky Bluegrass-Timothy/Dandelion

\section{(CMB2)}

Pasture very heavily grazed, weedy invaders common. Kentucky Bluegrass/Dandelion (CMB1) 


\section{CMB1. Kentucky bluegrass/Dandelion (Poa pratensis/Taraxacum officinale)}

$\mathbf{n}=\mathbf{8}$ This community type has had a history of being grazed heavily throughout the growing season. Heavy grazing throughout the growing season, allows Kentucky bluegrass, clover, and dandelion to out-compete all of the other vegetation. This community occurs on tame pastures or native rangeland that has been over grazed. It is considered to be in poor condition because forage production and nutritional value have been degraded

\section{Plant COMPOSITION CANOPY COVER(\%) \\ MEAN RANGE CONST.}

FORBS

CLOVER

(Trifolium spp.)

DANDELION

(Taraxacum officinale)

PLANTAIN

(Plantago major) 4

GRASSES

SMOOTH BROME

(Bromus inermis)

QUACKGRASS

(Agropyron repens)

TIMOTHY

(Phleum pratense)

KENTUCKY BLUEGRASS

(Poa pratensis)

$13 \quad 0-87 \quad 100$

$\begin{array}{lll}17 & 1-89 & 100\end{array}$

$0-26 \quad 25$

$\begin{array}{lll}6 & 0-17 \quad 62\end{array}$

$12 \quad 0-55 \quad 41$

$2 \quad 0-11 \quad 38$

$55 \quad 4-81 \quad 100$
ENVIRONMENTAL VARIABLES

MOISTURE REGIME (MEAN): MESIC-SUBHYGRIC

NUTRIENT REGIME (MEAN): MEDIUM TO RICH

ELEVATION:

$$
\text { 576-667(594)M }
$$

SOIL DRAINAGE (MEAN):

WELL TO MODERATELY WELL

RANGELAND HEALTH RATING:

UNHEALTHY

FORAGE PRODUCTION(KG/HA)

GRASS 2344(858-5304)

FORBS 843(0-3322)

TOTAL 2929(858-5371)

ECOLOGICALLY SUSTAINABLE STOCKING

RATE

$0.4 \mathrm{HA} / \mathrm{AUM}$ (1.0 AUM/AC) 


\section{CMB2. Kentucky bluegrass-Timothy/Dandelion (Poa pratensis-Phleum pratense/Taraxacum officinale)}

$\mathbf{n}=\mathbf{6}$ This community type represents a tame pasture that is showing signs of heavy grazing pressure. The original seed mix species (such as timothy and smooth brome) are still present, however Kentucky bluegrass and dandelion have become established and are starting to take over. If allowed some rest, this field may recover to the original seeded species.

PLANT COMPOSITION CANOPY COVER(\%) MEAN RANGE CONST.

FORBS

CLOVER

(Trifolium spp.)

$25 \quad 1-67 \quad 100$

DANDELION

(Taraxacum officinale) $\quad 36 \quad 23-47 \quad 100$

CANADA THISTLE

(Cirsium arvense)

HORSETAIL

$\begin{array}{llll}\text { (Equisetum arvense) } & 6 & 1-28 & 100\end{array}$

GRASSES

CREEPING RED FESCUE

(Festuca rubra)

FOXTAIL BARLEY

(Hordeum jubatum) $3 \quad 0-16 \quad 33$

KENTUCKY BLUEGRASS

(Poa pratensis)

TIMOTHY

(Phleum pratense)

SMOOTH BROME

(Bromus inermis)

$3 \quad 0-19 \quad 33$

$-100$

1

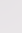

$39 \quad 10-59 \quad 100$

$11 \quad 1-25 \quad 100$

$2 \quad 0-12 \quad 33$

\section{ENVIRONMENTAL VARIABLES}

MOISTURE REGIME (MEAN): MESIC TO SUBHYGRIC

NUTRIENT REGIME (MEAN): MEDIUM TO RICH

ELEVATION: 576-606(596)M

SOIL DRAINAGE (MEAN): WELL TO MODERATELY WELL

RANGELAND HEALTH RATING:

HEALTHY WITH PROBLEMS

\section{FORAGE PRODUCTION(KG/HA)}

$$
\begin{array}{ll}
\text { GRASS } & 1396(700-2434) \\
\text { FORBS } & 1098(280-2042) \\
\text { SHRUB } & 17(0-100) \\
\text { TOTAL } & 2793(1300-3902)
\end{array}
$$

ECOLOGICALLY SUSTAINABLE STOCKING RATE $0.3 \mathrm{HA} / \mathrm{AUM}$ ( $1.0 \mathrm{AUM} / \mathrm{AC})$ 


\section{CMB3. Timothy/Dandelion (Phleum pratense / Taraxacum officinale)}

n=1 This community type develops on sites that were seeded with a timothy, meadow foxtail, clover mixture. Timothy establishes very quickly on pastures that have been recently seeded. If this pasture had been seeded with creeping red fescue and smooth brome they eventually will outcompete timothy and this community will likely become dominated by creeping red fescue and smooth brome. Timothy provides excellent forage for domestic livestock, especialy if timothy is kept in the vegetative state by mowing or grazing.

Plant COMPOSITION CANOPY COVER(\%) MEAN RANGE CONST.

\section{FORBS}

ALSIKE CLOVER

(Trifolium hybridum)

DANDELION

(Taraxacum officinale)

WILD STRAWBERRY

(Fragaria virginiana)

GRASSES

MEADOW FOXTAIL

(Alopecurus pratensis)

TIMOTHY

(Phleum pratense)

MEAN RANGE CONST.

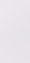

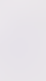

\section{ENVIRONMENTAL VARIABLES}

MOISTURE REGIME (MEAN): MESIC TO SUBHYGRIC

NUTRIENT REGIME (MEAN): MEDIUM TO RICH

ELEVATION: $576 \mathrm{M}$

SOIL DRAINAGE (MEAN): WELL (MODERATELY WELL)

RANGELAND HEALTH RATING: HEALTHY

FORAGE PRODUCTION(KG/HA)
ECOLOGICALLY SUSTAINABLE STOCKING RATE $0.4 \mathrm{HA} / \mathrm{AUM}(1.0 \mathrm{AUM} / \mathrm{AC})$ 


\section{CMB4. Creeping red fescue-Kentucky bluegrass/Dandelion (Festuca rubra-Poa pratensis/Taraxacum officinale)}

$\mathbf{n = 3}$ This community type represents recently seeded fields that have since been moderately to heavily grazed. Kentucky bluegrass has increased to equal the level of the seeded species, creeping red fescue. If heavy grazing pressure persists, Kentucky bluegrass and dandelion will dominate resulting in a decline in forage quality. This community type will require a period of rest each year with light to moderate grazing pressure to maintain good cover of creeping red fescue.

\section{PLANT COMPOSITION CANOPY COVER(\%)}

\section{SHRUBS}

PRICKLY ROSE

(Rosa acicularis) 3

FORBS

Clover

(Trifolium spp.)

DANDELION

(Taraxacum officinale)

AlFALFA

(Medicago sativa)

GRASSES

KENTUCKY BLUEGRASS

(Poa pratensis)

TIMOTHY

(Phleum pratense)

CREEPING RED FESCUE

(Festuca rubra)
$0-7 \quad 66$

\section{MEAN RANGE CONST.}

$0-7$

$\begin{array}{ccc}2 & 0-3 & 66 \\ 6 & 1-14 & 100 \\ 5 & 0-15 & 100 \\ 38 & 16-52 & 100 \\ 1 & 1-2 & 100 \\ 34 & 24-48 & 100\end{array}$

\section{ENVIRONMENTAL VARIABLES}

MOISTURE REGIME (MEAN):

MESIC

NUTRIENT REGIME (MEAN):

MEDIUM

ELEVATION:

$579(576-606) \mathrm{M}$

SOIL DRAINAGE (MEAN):

WELL

RANGELAND HEALTH RATING:

HEALTHY WITH PROBLEMS

FORAGE PRODUCTION(KG/HA)
GRASS $1859(846-2738)$
FORBS 2722(120-2348)
SHRUBS 0
TOTAL 2767(1100-5086)

\footnotetext{
ECOLOGICALLY SUSTAINABLE STOCKING RATE $0.3 \mathrm{HA} / \mathrm{AUM}(1.0 \mathrm{AUM} / \mathrm{AC})$
} 


\section{CMB5. Creeping red fescue-Rough hairgrass}

(Festuca rubra-Agrostis scabra)

$\mathbf{n = 1} \quad$ This community type represents an area that was cleared and seeded, however due to poor soil conditions, it established poorly. The soils on this site are sandy to a depth of about 6 inches and hairgrass is well adapted to growing on these disturbed sites with poor nutrients. The overall cover of vegetation is sparse, therefore grazing should only be light in order to maintain the little cover of vegetation. This site should not have been approved for range improvement.

\section{PlaAt COMPOSITION CANOPY COVER(\%)}

\section{MEAN RANGE CONST.}

FORBS

THREE TOOTHED CINQUEFOIL

(Potentilla tridentata) 3

ROUGH CINQUEFOIL

(Potentilla norvegica)

\section{GRASSES}

ROUGH HAIRGRASS

(Agrostis scabra)

TIMOTHY

(Phleum pratense)

CREEPING RED FESCUE

(Festuca rubra)

1

$3 \quad-\quad 100$

$1 \quad-\quad 100$

$2 \quad-\quad 100$

$12 \quad-\quad 100$

\section{ENVIRONMENTAL VARIABLES}

MOISTURE REGIME (MEAN):

SUBMESIC

NUTRIENT REGIME (MEAN):

POOR

ELEVATION:

$579 \mathrm{M}$

SOIL DRAINAGE (MEAN):

WELL

RANGELAND HEALTH RATING:

UNHEALTHY

FORAGE PRODUCTION(KG/HA)

ECOLOGICALLY SUSTAINABLE STOCKING RATE

$0.8 \mathrm{HA} / \mathrm{AUM}(0.2 \mathrm{AUM} / \mathrm{AC})$ 


\section{CMB6. Creeping red fescue-Timothy/Dandelion \\ (Festuca rubra-Phleum pratense/Taraxacum officinale)}

$\mathbf{n}=\mathbf{9} \quad$ This community type represents areas cleared of aspen, seeded to timothy, creeping red fescue and clover. In general, these sites are in good condition, but they will require adequate rest periods from grazing each season to remain in good condition.

PlaNT COMPOSITION CANOPY COVER(\%)

MEAN RANGE CONST.

FORBS

CLOVER

(Trifolium spp.)

DANDELION

(Taraxacum officinale)

COMMON YARROW

(Achillea millefolium)

GRASSES

CREEPING RED FESCUE

(Festuca rubra)

KENTUCKY BLUEGRASS

(Poa pratensis)

TIMOTHY

(Phleum pratense)

$\begin{array}{lll}10 & 0-26 & 97 \\ 7 & 0-14 & 78 \\ 1 & 0-3 & 56 \\ 50 & 25-79 & 100 \\ 3 & 0-8 & 78 \\ 6 & 0-19 & 78\end{array}$

\section{ENVIRONMENTAL VARIABLES}

MOISTURE REGIME (MEAN):

MESIC

NUTRIENT REGIME (MEAN):

MEDIUM

ELEVATION:

576-636(603)M

SOIL DRAINAGE (MEAN):

WELL

RANGELAND HEALTH RATING:

HEALTHY

FORAGE PRODUCTION(KG/HA)

GRASS 1328(724-2588)

FORB $750(250-1590)$

SHRUB 19(0-162)

TOTAL 2097(1214-3268)

ECOLOGICALLY SUSTAINABLE STOCKING RATE $0.4 \mathrm{HA} / \mathrm{AUM}$ ( $1.0 \mathrm{AUM} / \mathrm{AC})$ 


\section{CMB7. Smooth brome (Bromus inermis)}

$\mathbf{n}=\mathbf{2}$ This community type occurs on cleared pastures that were seeded with a smooth brome, timothy-clover mix. With a moderate grazing regime, smooth brome grass is the most competitive forage plant seeded into this site. It out-competes timothy because it is strongly rhizomatous and is able to fill in the gaps between plants allowing it to shade out the timothy. One of the sites had only been recently cleared and the forage species had not yet fully established. As a result, forage production was only $1 / 3$ as high as expected.

\section{PlaANT COMPOSITION Canopy Cover(\%)} MEAN RANGE CONST.

\section{SHRUBS}

PRICKLY ROSE

(Rosa acicularis)

FORBS

Clover

(Trifolium spp.)

$2 \quad 0-3 \quad 100$

GRASSES

SMOOTH BROME

(Bromus inermis)

SEDGE SPP.

(Carex spp.)

TIMOTHY

(Phleum pratense)

KENTUCKY BLUEGRASS

(Poa pratensis)

MEAN RANGE CONST.

\section{ENVIRONMENTAL VARIABLES}

MOISTURE REGIME (MEAN):

MESIC

NUTRIENT REGIME (MEAN):

MEDIUM

ELEVATION:

$576 \mathrm{M}$

SOIL DRAINAGE (MEAN):

WELL (MODERATELY WELL)

RANGELAND HEALTH RATING:

HEALTHY

FORAGE PRODUCTION(KG/HA)

GRASS 2034

FORBS 680

TOTAL 2714

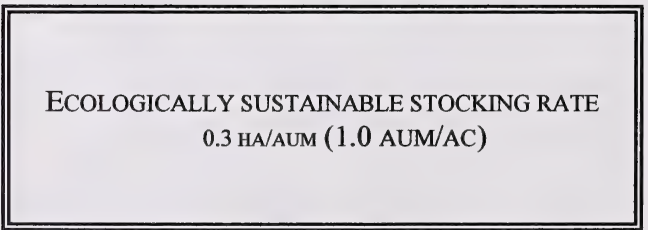




\section{CENTRAL MIXEDWOOD SUBREGION}

\section{DECIDUOUS FOREST COMMUNITY TYPES}

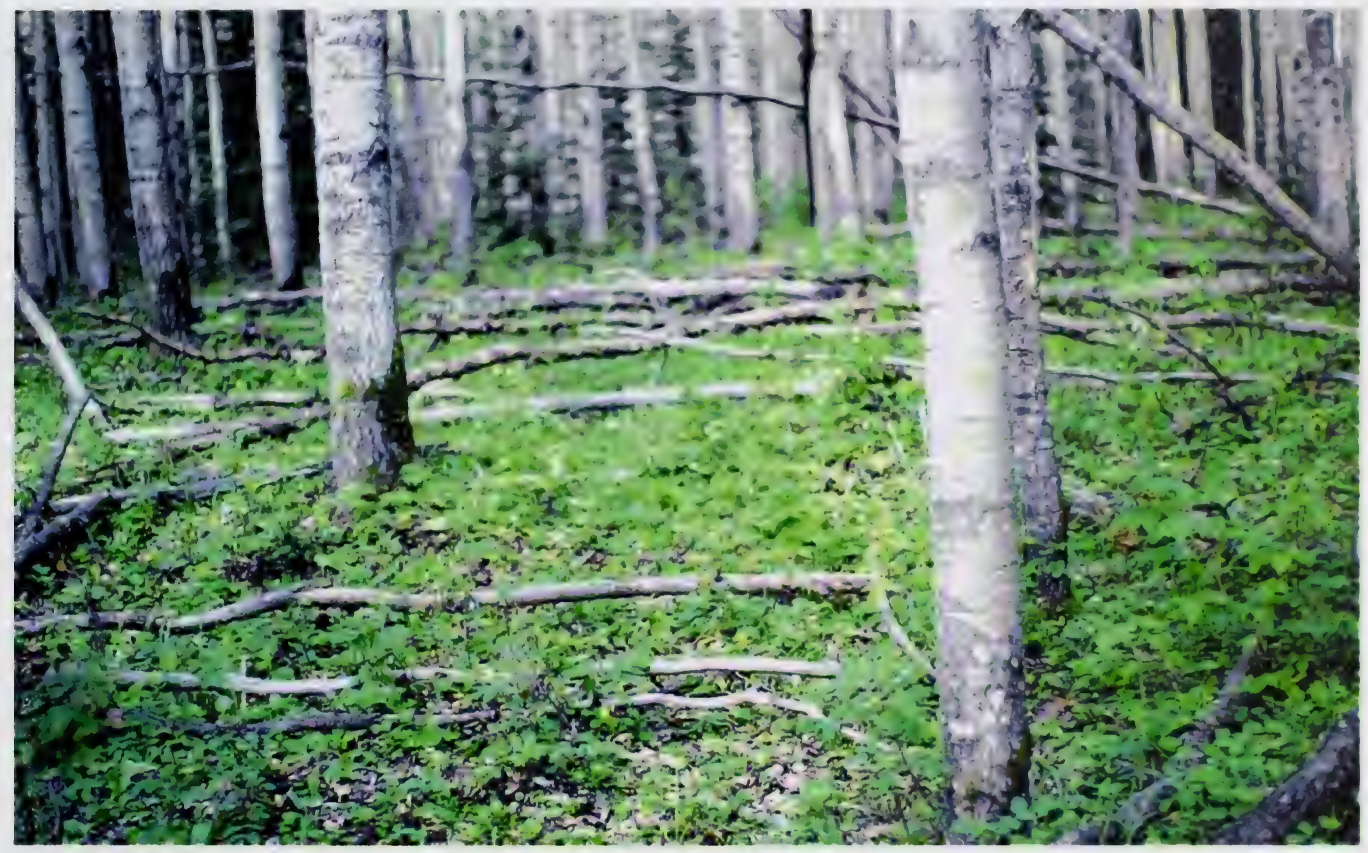

Photo 9. Aw/Rose/Clover community type represents a deciduous community that has been moderately to heavily grazed for a number of years. 


\section{CM - DECIDUOUS FOREST COMMUNITIES}

Balsam poplar is most commonly found on moist upland and alluvial bottomland sites; its best growth is on moist rich bottomlands with deep soil (Peterson and Peterson 1992). The nine stands with predominant balsam poplar $(\mathrm{Pb})$ cover represent four community types in the Central Mixedwood subregion. The $\mathrm{Pb}-\mathrm{Aw} / \mathrm{River}$ alder community is found on lower slope positions and stream channels where there is seepage throughout the growing season. The $\mathrm{Pb} / \mathrm{Rose}-\mathrm{Alder}$, $\mathrm{Pb}-\mathrm{Aw} / \mathrm{Beaked}$ hazelnut-Rose and $\mathrm{Aw}-\mathrm{Pb} / \mathrm{Honeysuckle} \mathrm{community} \mathrm{types} \mathrm{are} \mathrm{found} \mathrm{upslope} \mathrm{on}$ slightly drier and better drained soils. These three community types integrade into the Aw/Rose dominated community types on mesic/medium ecosites.

White birch is indicative of well-drained, sandy or silty loams (Wilkinson 1990). In Alberta this tree is found in association with balsam poplar on moist sites adjacent to small creeks and lowland areas. Pure stands of Alaska variety white birch are also found on dry sandy ridges with high watertables throughout northern Alberta. Beckingham (1993), found that white birch was well adapted to growing on a soil with a pH of less than 5.3. The White birch/Willow dominated community type maybe indicative of sites with slightly lower $\mathrm{pH}$ 's.

More mesic sites tend to be dominated by aspen and rose. It is the underlying soil conditions and site history that appear to dictate which forb and shrub species will dominate these mesic sites. Blueberry and twinflower appear to indicate sandy soils with poorer nutrient regimes. An abundance of tall forbs (Aw/Rose/Tall forb) appears to be indicative of higher nutrient regimes that have not been disturbed by livestock. In contrast the low forb (Aw/Rose/Low forb) dominated type occupies sites similar to the tall forb type, but these sites appear to have been disturbed by livestock. Increased grazing pressure on these two community types leads to the formation of strawberry and clover dominated community types $(\mathrm{Pb}-$ Aw/Rose/Strawberry, Aw/Rose/Clover).

Sites that have a more subhygric moisture regime and are moderately well-drained tend to be dominated by willow and alder (Aw/Alder-Willow-Rose, Aw/Willow). The Aw/RoseSaskatoon community was described on south and west facing slopes overlooking streams and rivers. This community is very similar to the community that was described in the Dry Mixedwood subregion. On sites with rich nutrient regimes red osier dogwood and horsetail dominated communities are very common. The Aw/Horsetail community is usually found on moister sites than the $\mathrm{Aw}-\mathrm{Pb} / \mathrm{Red}$ osier dogwood-Rose community type. 


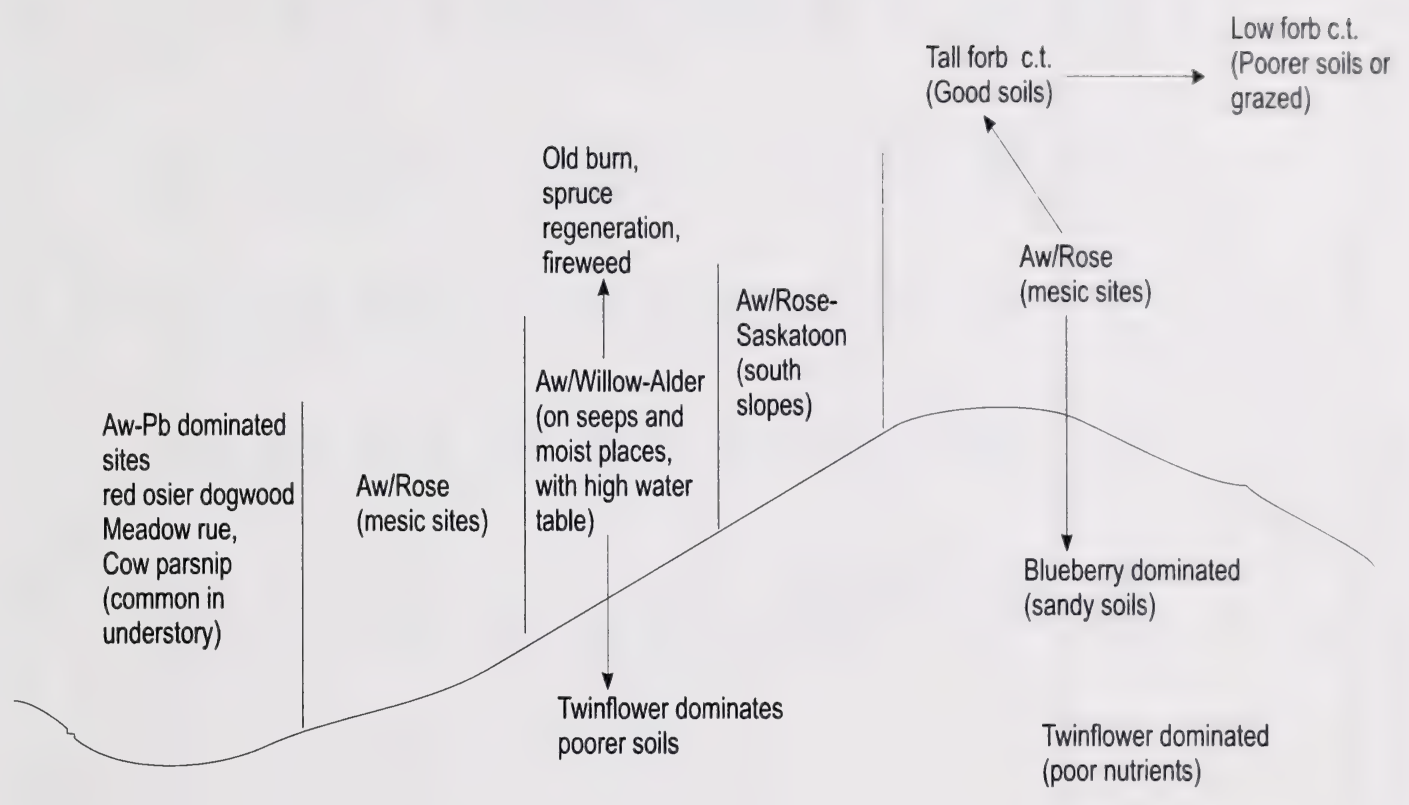

Figure 4 Sequence of Aspen/Rose dominated community types in the landscape of the Central Mixedwood subregion. 


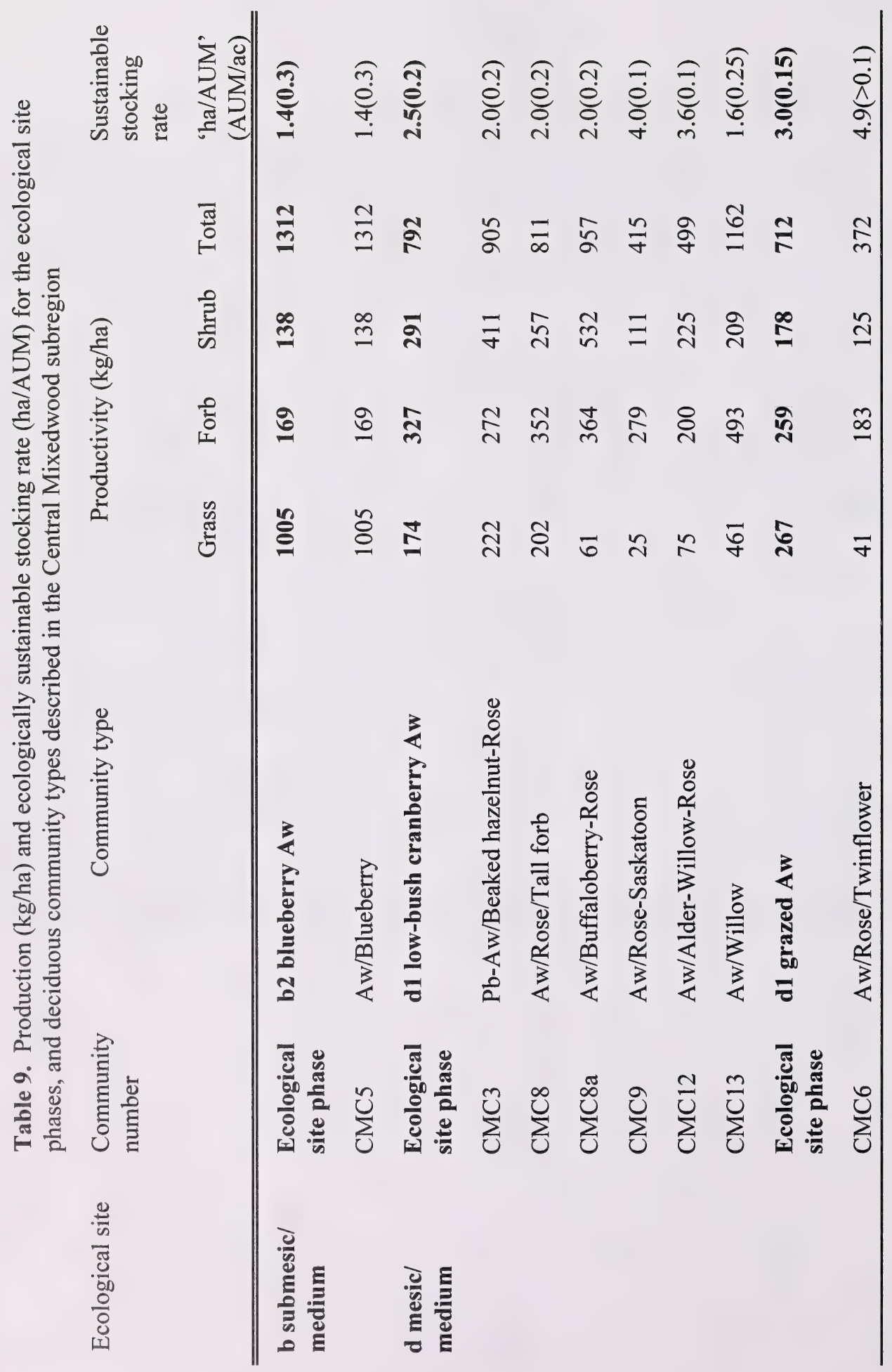

壱 


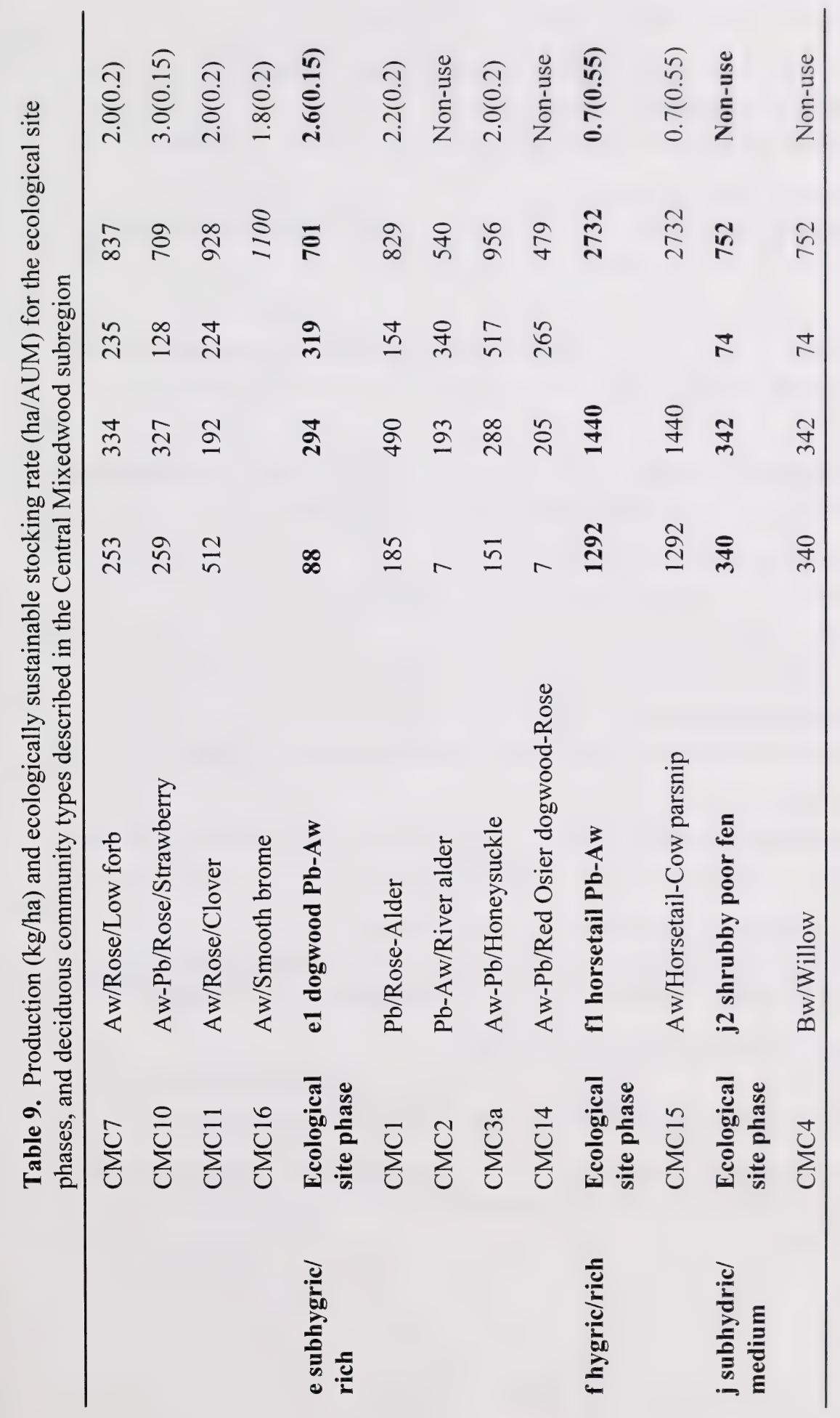

$\stackrel{\circ}{\circ}$ 


\section{Deciduous Community Types - Central Mixedwood Subregion}

1. Community dominated by balsam poplar or birch, richer, moister sites (aspen may be present but is only co-dominant). 2

Community dominated by aspen, mesic sites.

2. Community dominated by paper birch, with willow understory. Bw/Willow (CMC4)

Community dominated by $\mathrm{Pb}, \mathrm{Bw}$ and $\mathrm{Aw}$ only minor.

3. Understory dominated by hazelnut, mesic sites. Pb-Aw/Beaked Hazelnut-Rose (CMC3)

Community dominated by green or river

alder, honeysuckle or red osier dogwood.

4. Community understory dominated by honeysuckle Aw-Pb/Honeysuckle (CMC3a)

Community dominated by river or green alder or red osier dogwood.

5. Community dominated by river or green alder.

Community dominated by red osier dogwood, fluvial floodplains next to rivers.

6. Community dominated by green alder, more upland sites with mesic moisture regimes Pb/Rose-Alder (CMC1) Community dominated by river alder, moist seepage areas next to rivers. ..Pb-Aw/River Alder (CMC2)

7. Willow, alder dominate the understory. 8 Rose, red osier dogwood, saskatoon, buffaloberry, red raspberry and blueberry dominate understory.

8. Willow dominates understory, alder cover very small, fire origin. Aw/Willow (CMC13) Alder dominates understory, fire origin Aw/Alder-Willow-Rose (CMC12)

9. Blueberry dominates shrub layer, rose is co-dominant, dry, sandy soils. Aw/Blueberry (CMC5) Mesic or richer sites dominated by buffaloberry, rose, saskatoon, red osier dogwood.....10

10. Buffaloberry dominates shrub understory Rose, red raspberry, saskatoon, or red-osier dogwood dominate shrub layer. Aw/Buffaloberry-Rose (CMC8a) 
11. Red osier dogwood is dominant shrub, rose is co-dominant, community type found on rich fluvial floodplains adjacent to river or stream Aw-Pb/Red Osier Dogwood-Rose (CMC14) Rose, red raspberry or saskatoon dominate shrub layer.

12. Saskatoon is dominant shrub with rose, community type found on south facing slopes above rivers and streams Aw/Rose-Saskatoon (CMC9) Rose or red raspberry is dominant shrub.

13. Community not modified appreciably by grazing (tall forb dominated) Community moderately to severely modified by grazing (low forb dominated) or poorer nutrient sites which are dominated by twinflower or smooth brome.

14. Mesic sites, forb layer dominated by tall forbs, wild sarsaparilla, showy aster, fireweed, peavine Aw/Rose/Tall Forb (CMC8) Nutrient rich seepage areas, little rose cover, red raspberry dominant shrub, understory dominated by horsetail and cow parsnip, moist type associated with willow lowlands Aw/Horsetail-Cow Parsnip (CMC15)

15. Clover common in understory Aw/Rose/Clover (CMC11) Clover not common in understory (low forb or smooth brome dominated).

16. Twinflower dominates forb layer, poorer soils Aw/Rose/Twinflower (CMC6) Other low forbs or smooth brome dominate understory (bunchberry, wintergreen, strawberry, wild lily of the valley).

17. Moderately grazed, $\mathrm{Pb}$ in overstory. Aw-Pb/Rose/Strawberry (CMC10) Aspen dominated, forb layer dominated by low forbs, smooth brome strawberry, bunchberry, wintergreen, etc., site partially grazed,low cover of shrubs 18

18. Native understory dominated by low growing forbs...Aw/Rose/Low forb (CMC7) Smooth brome dominates the understory..... Aw/Smooth brome (CMC16) 


\section{CMC1. Pb/Alder-Rose \\ (Populus balsamiferal Alnus crispa- Rosa acicularis)}

$\mathbf{n = 4}$ This community was found on moderately well-drained sites with subhygric moisture regimes. Beckingham (1993), described a similar community type. He found these forests to develop on parent materials that are neutral to alkaline, thus they tended to have a relatively high level of nutrient availability and potentially high production levels.

This commmunity is producing only a moderate forage base for domestic livestock. Green alder, which makes up a large part of the total forage production for this vegetation type, is generally unpalatable to livestock. This community type would be rated as secondary or non-use range.

\section{Plant Composition Canopy Cover $(\%)$ \\ MEAN RANGE CONST.}

\section{TreES}

TREMBLING ASPEN

(Populus tremuloides)

BALSAM POPLAR

(Populus balsamifera) $\quad 50 \quad 10-65 \quad 100$

SHRUBS

PRICKLY ROSE

(Rosa acicularis)

GREEN ALDER

(Alnus crispa)

LOW BUSH CRANBERRY

(Viburnum edule)

FORBS

STRAWBERRY

(Fragaria virginiana)

TWINFLOWER

(Linnaea borealis)

NORTHERN BEDSTRAW

(Galium boreale)

TALL LUNGWORT

(Mertensia paniculata)

YELLOW PEAVINE

(Lathyrus ochroleucus)

WILD SARSAPARILLA

(Aralia nudicaulis)

GRASSES

MARSH REEDGRASS

(Calamagrostis canadensis)6
$13 \quad 6-16 \quad 100$

$21 \quad 12-40 \quad 100$

$8 \quad 0-16 \quad 75$

$5 \quad 1-10 \quad 100$

$1 \quad 0-4 \quad 75$

$4 \quad 0-8 \quad 75$

$\begin{array}{lll}4 & 3-7 & 100\end{array}$

$1 \quad 0-2 \quad 25$

$2-8 \quad 100$
$4 \quad 1-6 \quad 100$

\section{ENVIRONMENTAL VARIABLES}

MOISTURE REGIME:

SUBHYGRIC

NUTRIENT REGIME:

MESOTROPHIC

ELEVATION:

$567 \mathrm{M}$

SOIL DRAINAGE:

MODERATELY WELL

RANGELAND HEALTH RATING:

HEALTHY

\section{FORAGE PRODUCTION(KG/HA)}

GRASS $\quad 185(0-552)$

FORBS 490(234-978)

SHRUBS 154(0-250)

TOTAL 829(474-1530)

ECOLOGICALLY SUSTAINABLE STOCKING RATE 2.2 HA/AUM(0.2 AUM/AC) 


\section{CMC2. Pb-Aw/River alder \\ (Populus balsamifera-Populus tremuloides/Alnus tenuifolia)}

$\mathbf{n = 4}$ This community type is found on moist lower slope positions. A similar community type was described on similar sites in the Lower Foothills subregion (Willoughby and Downing 1995). The high cover of alder limits the light reaching the understory and results in low production of grass and forbs. The majority of the total forage production comes from alder which is generally inaccessible and unpalatable to livestock. Consequently this community type would be rated as non-use for domestic livestock.

Plant COMPOSITION CANOPY COVER(\%) MEAN RANGE CONST.

TREES

TREMBLING ASPEN

(Populus tremuloides) $23 \quad 0-35 \quad 75$

BALSAM POPLAR

(Populus balsamifera) $\quad 26 \quad 19-45 \quad 100$

SHRUBS

RIVER ALDER

(Alnus tenuifolia)

RED OSIER DOGWOOD

(Cornus stolonifera) $\quad 10 \quad 5-17 \quad 100$

BRACTED HONEYSUCKLE

(Lonicera involucrata) $3 \quad 0-10 \quad 25$

PRICKLY ROSE

$\begin{array}{llll}\text { (Rosa acicularis) } & 9 & 4-18 & 100\end{array}$

LOW BUSH CRANBERRY

$\begin{array}{llll}\text { (Viburnum edule) } & 4 & 1-10 & 100\end{array}$

\section{FORBS}

HORSETAIL

(Equisetum arvense) $\quad 14 \quad 1-45 \quad 100$

DEWBERRY OR RUNNING RASPBERRY

$\begin{array}{llll}\text { (Rubus pubescens) } & 6 & 1-8 & 100\end{array}$

BISHOP'S CAP

(Mitella nuda)

$\begin{array}{lll}4 & 0-7 & 75\end{array}$

WILD STRAWBERRY

$\begin{array}{llll}\text { (Fragaria virginiana) } & 3 & 2-4 & 75\end{array}$

LINDLEY'S ASTER

(Aster ciliolatus)

YELLOW PEAVINE

(Lathyrus ochroleucus)

WILD SARSAPARILLA

(Aralia nudicaulis)

$2 \quad 2-4 \quad 75$

$3 \quad 2-5 \quad 75$

GRASSES

$2 \quad 0-7 \quad 50$

MARSH REED GRASS

(Calamagrostis canadensis)

\section{ENVIRONMENTAL VARIABLES}

MOISTURE REGIME:

SUBHYGRIC TO HYGRIC

NUTRIENT REGIME:

PERMESOTROPHIC

ELEVATION:

454(150-606) M

PERCENT SLOPE GRADIENT:

0 - 2

SOIL DRAINAGE:

MODERATELY WELL

RANGELAND HEALTH RATING:

HEALTHY

\section{FORAGE PRODUCTION(KG/HA)}

GRASS 7(2-20)

FORBS 193(62-376)

SHRUBS $\quad 340(200-438)$

TOTAL 540(202-816)

ECOLOGICALLY SUSTAINABLE STOCKING RATE NON-USE 


\section{CMC3. Pb-Aw/Beaked hazelnut-Rose \\ (Populus balsamifera-Populus tremuloides/Corylus cornuta-Rosa acicularis)}

$\mathbf{n}=2$ This community type was described on south facing slopes and is very similar to the the beaked hazelnut communities described in the Dry Mixedwood subregion. This type appears to occupy warmer and drier microsites that resemble the Dry Mixedwood's climate. The total production of this type is high, but the majority of production is coming from hazelnut which is largely unpalatable to livestock at proper stocking levels. The high cover of hazelnut also restricts access to livestock, limiting the forage availability. This community would be rated as secondary range.

Plant COMPOSITION CANOPY COVER(\%) MEAN RANGE CONST.

TREES

TREMBLING ASPEN

(Populus tremuloides)

BALSAM POPLAR

(Populus balsamifera) $33 \quad 0-65 \quad 50$

SHRUBS

HAZELNUT

(Corylus cornuta)

$23 \quad 13-32 \quad 100$

SASKATOON

(Amelanchier alnifolia)

WILD RED RASPBERRY

(Rubus idaeus)

PRICKLY ROSE

(Rosa acicularis)

FORBS

WILD LILY-OF-THE-VALLEY

(Maianthemum canadense) $5 \quad 1-8 \quad 100$

DEWBERRY OR RUNNING RASPBERRY

(Rubus pubescens)

YELLOW PEAVINE

$\begin{array}{llll}\text { (Lathyrus ochroleucus) } & 4 & 1-6 & 100\end{array}$

VEINY MEADOW RUE

$\begin{array}{llll}\text { (Thalictrum venulosum) } & 3 & 2-3 & 100\end{array}$

WILD SARSAPARILLA

(Aralia nudicaulis)

$11 \quad 0-23 \quad 50$

GRASSES

MARSH REED GRASS

(Calamagrostis canadensis)5 $\quad 0-9 \quad 50$

MOUNTAIN RICEGRASS

(Oryzopsis asperifolia) $\quad 3 \quad 0-7 \quad 50$

SEDGE

(Carex spp.)

$5 \quad 0-10$

\section{ENVIRONMENTAL VARIABLES}

MOISTURE REGIME:

MESIC TO SUBHYGRIC

NUTRIENT REGIME:

MESOTROPHIC TO PERMESOTROPHIC

ELEVATION:

588(576-600) M

PERCENT SLOPE GRADIENT:

$5-10(7.5) \%$

SOIL DRAINAGE:

WELL TO MODERATELY WELL

RANGELAND HEALTH RATING:

HEALTHY

\section{FORAGE PRODUCTION(KG/HA)}

GRASS

222(74-370)

FORBS 272(234-310)

SHRUBS 411(152-670)

TOTAL 905(756-1054)

ECOLOGICALLY SUSTAINABLE STOCKING RATE

$2.0 \mathrm{HA} / \mathrm{AUM}(0.2 \mathrm{AUM} / \mathrm{AC})$ 


\section{CMC3a. Aw-Pb/Honeysuckle \\ (Populus tremuloides- Populus balsamifera /Lonicera involucrata)}

$\mathbf{n}=\mathbf{6}$ This community type is represented by one of the Public Lands Peace River benchmark sites. It is a relatively moist and nutrient rich site and represents the honeysuckle ecosite as described by Beckingham and Archibald (1996). The high tree and shrub layer limit the amount of light reaching the forest floor. Consquently. there is little growth of grasses and forbs. Shrub production is largely honeysuckle which is generally unpalatable to domestic livestock. This community type should be rated as secondary range.

\section{PLANT COMPOSITION CANOPY COVER(\%)} MEAN RANGE CONST.

TREES

TREMBLING ASPEN

(Populus tremuloides)

BALSAM POPLAR

$\begin{array}{llll}\text { (Populus balsamifera) } & 57 & 2-90 & 100\end{array}$

SHRUBS

HONEYSUCKLE

(Lonicera involcrata)

RED OSIER DOGWOOD

(Cornus stolonifera)

WILD RED RASPBERRY

(Rubus idaeus)

PRICKLY ROSE

(Rosa acicularis)

FORBS

FIREWEED

(Epilobium angustifolium)

DEWBERRY OR RUNNING RASPBERRY

(Rubus pubescens) 2

(Petasites palmatus) 1

$9 \quad 0-31$

83

$4 \quad 0-10$

83

$4 \quad 0-13 \quad 83$

$14 \quad 3-20$

100

TALL LUNGWORT

(Mertensia paniculata)

WILD SARSAPARILLA

(Aralia nudicaulis)

$8 \quad 0-20$

GRASSES

MARSH REED GRASS

(Calamagrostis canadensis) 9
ENVIRONMENTAL VARIABLES

MOISTURE REGIME: SUBHYGRIC

NUTRIENT REGIME: PERMESOTROPHIC

ELEVATION:

$869 \mathrm{M}$

SOIL DRAINAGE:

WELL TO MODERATELY WELL

RANGELAND HEALTH RATING:

HEALTHY

\section{FORAGE PRODUCTION(KG/HA)}

GRASS

151

FORBS $\quad 288$

SHRUBS $\quad 517$

TOTAL $\quad 956$

ECOLOGICALLY SUSTAINABLE STOCKING RATE

$2.0 \mathrm{HA} / \mathrm{AUM}(0.2 \mathrm{AUM} / \mathrm{AC})$ 


\section{CMC4. Bw/Willow \\ (Betula papyrifera/Salix spp.)}

$\mathbf{n = 1} \quad$ This community type was described on a very moist site that was burned or cleared and is now undergoing succession to a paper birch dominated community type. The understory of this community type is dominated by sphagnum moss, which is characteristic of the poor fen ecosite described by Beckingham and Archibald (1996). The site was likely dominated by black spruce and larch prior to disturbance. The poor nutrient status and very moist conditions make this community type unsuitable for livestock grazing. This community type should be rated as nonuse.

Plant COMPOSITION CANOPY COVER(\%) MEAN RANGE CONST.

TREES

PAPER BIRCH

(Betula papyrifera)

55

SHRUBS

WILLOW

(Salix spp.)

50

100

FORBS

SMALL BOG CRANBERRY

(Oxycoccus microcarpus) 25

SWAMP HORSETAII

(Equisetum fluviatile)

MARSH CINQUEFOIL

(Potentilla palustris)

$19 \quad-$

12

100

GRASSES

MARSH REED GRASS

(Calamagrostis canadensis) 7

HAIR-LIKE SEDGE

(Carex capillaris)

BEAKED SEDGE

(Carex rostrata)

6

6

Mosses

PEAT MOSS

(Sphagnum spp.)

$93 \quad 100$

\section{ENVIRONMENTAL VARIABLES}

MOISTURE REGIME:

SUBHYDRIC

NUTRIENT REGIME:

MESOTROPHIC

ELEVATION:

$576 \mathrm{M}$

SOIL DRAINAGE:

VERY POOR

RANGELAND HEALTH RATING:

HEALTHY

FORAGE PRODUCTION(KG/HA)

GRASS $\quad 340$

FORBS $\quad 342$

SHRUBS $\quad 74$

TOTAL $\quad 756$

ECOLOGICALLY SUSTAINABLE STOCKING RATE

NON-USE 


\section{CMC5. Aw/Blueberry \\ (Populus tremuloides/Vaccinium myrtilloides)}

$\mathbf{n = 4} \quad$ This is a very dry, well-drained community type with sandy soil. It is found in conjunction with jack pine

stands. Productivity of shrubs is largely blueberry, which is unpalatable to livestock.

These stands tend to be relatively open allowing for easy access by livestock, but the dry site conditions and poorer nutrient status limit the amount of regrowth after grazing. If this community type is managed for one rotation a year, it can contribute significantly to the overall carrying capacity of a lease.

\section{PLANT COMPOSITION CANOPY COVER(\%)} MEAN RANGE CONST.

\section{TREES}

TREMBLING ASPEN

(Populus tremuloides) $\quad 45 \quad 35-60 \quad 100$

\section{SHRUBS}

WILLOW

(Salix spp.)

$5 \quad 0-20 \quad 50$

WILD RED RASPBERRY

(Rubus idaeus)

BLUEBERRY

(Vaccinium myrtilloides)

PRICKLY ROSE

(Rosa acicularis)

FORBS

BUNCHBERRY

(Cornus canadensis) 7

BEARBERRY

(Arctostaphylos uva-ursi) 3

YELLOW PEAVINE

(Lathyrus ochroleucus) 4

TWINFLOWER

(Linnaea borealis) $\quad 4 \quad 0-7 \quad 75$

STRAWBERRY

$\begin{array}{llll}\text { (Fragaria virginiana) } & 4 & 1-5 & 100\end{array}$

GRASSES

PURPLE OATGRASS

(Schizachne purpurascens)3 $\quad 0-7 \quad 75$

HAIRY WILDRYE

(Elymus innovatus)

MOUNTAIN RICEGRASS

(Oryzopsis asperfolia)
$3 \quad 1-6 \quad 100$

$5 \quad 0-10 \quad 75$

\section{ENVIRONMENTAL VARIABLES}

MOISTURE REGIME:

SUBMESIC

NUTRIENT REGIME:

MESOTROPHIC

ELEVATION:

604(600-606) M

SOIL DRAINAGE:

WELL

RANGELAND HEALTH RATING:

HEALTHY

FORAGE PRODUCTION(KG/HA)

GRASS 1005(98-1794)

FORBS 169(0-388)

SHRUBS 138(0-452)

TOTAL 1312(762-1794)

ECOLOGICALLY SUSTAINABLE STOCKING RATE

1.4 HAVAUM (0.3 AUM/AC) 


\section{CMC6. Aw/Rose/Twinflower \\ (Populus tremuloides/Rosa acicularis/Linnaea borealis)}

$\mathbf{n}=6$ This community type has been grazed moderately to heavily and is very similar to the grazed $\mathrm{Pb} /$ Rose/Strawberry and Aw/Rose/Low forb community types. Grazing pressure reduces the cover of shrubs and tall-growing forbs and allows the low-growing forbs to increase in cover. This community type occupies soils with poor nutrient regimes. The poor nutrient status appears to favour the growth of twinflower, a species that is well adapted to growing on poor soils (Corns and Annas 1986). This may explain why twinflower is predominant on this community type and not on the other grazed community types.

\section{Plant COMPOSITION CANOPY COVER(\%)} MEAN RANGE CONST.

\section{TREES}

BALSAM POPLAR

(Populus balsamifera) $\quad 3 \quad 0-15 \quad 17$

TREMBLING ASPEN

(Populus tremuloides) $\quad 53 \quad 25-75 \quad 100$

SHRUBS

PRICKLY ROSE

(Rosa acicularis)

WILD RED RASPBERRY

$\begin{array}{llll}\text { (Rubus idaeus) } & 1 & 0-1 & 50\end{array}$

LOW BUSH CRANBERRY

(Viburnum edule) 2

SNOWBERRY OR BUCKBRUSH

(Symphoricarpos

occidentalis)

$3 \quad 1-5 \quad 100$

FORBS

CREAM-COLOURED VETCHLING

(Lathyrus ochroleucus) 3

BUNCHBERRY

(Cornus canadensis) $\quad 5 \quad 1-10 \quad 100$

TWINFLOWER

(Linnaea borealis) $\quad 19 \quad 11-31 \quad 100$

STRAWBERRY

$\begin{array}{llll}\text { (Fragaria virginiana) } & 5 & 2-9 & 100\end{array}$

WINTER GREEN

(Pyrola asarifolia)

$3 \quad 1-7 \quad 100$

GRASSES

MOUNTAIN RICEGRASS

(Oryzopsis asperifolia)

HAIRY WILDRYE

(Elymus innovatus)

PURPLE OATGRASS

(Schizachne purpurascens) 3

KENTUCKY BLUEGRASS

(Poa pratensis)

\section{ENVIRONMENTAL VARIABLES}

MOISTURE REGIME:

SUBMESIC TO MESIC

NUTRIENT REGIME:

MESOTROPHIC

ELEVATION:

634(579-733) M

PERCENT SLOPE GRADIENT:

$2(0-5) \%$

SOIL DRAINAGE:

WELL

RANGELAND HEALTH RATING:

HEALTHY WITH PROBLEMS

\section{FORAGE PRODUCTION(KG/HA)}

GRASS 56(6-134)

FORBS 230(70-464)

SHRUBS 120(16-294)

TOTAL 406(190-692)

ECOLOGICALLY SUSTAINABLE STOCKING RATE

$4.9 \mathrm{HA} / \mathrm{AUM}(>0.1 \mathrm{AUM} / \mathrm{AC})$ 


\section{CMC7. Aw/Rose/Low forb \\ (Populus tremuloides/Rosa acicularis/Low forb)}

$\mathbf{n = 1 5}$ This type occupies mesic, well-drained sites with medium nutrient regimes. This type is similar to the Aw/Rose/Tall forb community type, but this type appears to occupy drier sites with poorer nutrient regimes. It has also been observed that this type can also be produced when the tall forb community is grazed for a number of years. The increased grazing pressure may explain why the production on this type is lower than the tall forb type. Forage production in this type is good, but the low-growing forbs are not as accessible to livestock as the tall growing forbs. Despite these limitations this community type should still be rated as primary range.

\section{Plant COMPOSITION CANopy COVER(\%)} MEAN RANGE CONST.

\section{TREES}

TREMBLING AsPEN

(Populus tremuloides)

BALSAM POPLAR

(Populus balsamifera) $\quad 8 \quad 0-20-53$

WHITE SPRUCE

(Picea glauca)

SHRUBS

SASKATOON

(Amelanchier alnifolia)

PRICKLY ROSE

(Rosa acicularis) $16 \quad 4-42 \quad 100$

WILD RED RASPBERRY

(Rubus idaeus)

6

SNOWBERRY

(Symphoricarpos

occidentalis)

$6 \quad 0-23 \quad 73$

LOW BUSH CRANBERRY

(Viburnum edule)

\section{FORBS}

STRAWBERRY

(Fragaria virginiana)

FIREWEED

(Epilobium angustifolium) 3

$8 \quad 0-36 \quad 80$

YELLOW PEAVINE

(Lathyrus ochroleucus) $\quad 3 \quad 0-11 \quad 73$

DEWBERRY OR RUNNING RASPBERRY

(Rubus pubescens)

(Aster ciliolatus)

GRASSES

$4 \quad 0-10 \quad 73$

$3 \quad 0-8 \quad 73$

MARSH REED GRASS

(Calamagrostis canadensis)6

HAIRY WILDRYE
(Elymus innovatus) $3 \quad 0-12 \quad 53$

\section{ENVIRONMENTAL VARIABLES}

MOISTURE REGIME:

MESIC

NUTRIENT REGIME:

MESOTROPHIC

ELEVATION:

617(579-667) M

PERCENT SLOPE GRADIENT:

$3(0-15) \%$

ASPECT:

VARIABLE

SOIL DRAINAGE:

WELL TO MODERATELY WELL

RANGELAND HEALTH RATING:

HEALTHY WITH PROBLEMS

FORAGE PRODUCTION(KG/HA)

$\begin{array}{ll}\text { GRASS } & 250(6-660) \\ \text { FORBS } & 335(76-830) \\ \text { SHRUBS } & 270(38-1154) \\ \text { TOTAL } & 842(312-2086)\end{array}$

ECOLOGICALLY SUSTAINABLE STOCKING RATE $2.0 \mathrm{HA} / \mathrm{AUM}(0.2 \mathrm{AUM} / \mathrm{AC})$ 


\section{CMC8. Aw/Rose/Tall forb \\ (Populus tremuloides/Rosa acicularis/Tall forb)}

$\mathbf{n = 1 7}$ This type appears to be the modal aspen community type in the absence of disturbance on mesic, medium to rich sites. The presence of tall forbs wild sarsaparilla, fireweed, and peavine distinguish this community from the low forb type. It is unclear why there is a difference in the tall and low forb types. Corns and Annas (1986) recoqnized the two types in the Lower Foothills subregion. They felt the wild sarsaparilla type was moister and had a higher nutrient regime. It has also been observed that the low forb type can be produced when the tall forb community is lightly to moderately grazed for a number of years (Willoughby 1996).

The forage production on this community type is good. The majority of the vegetation is palatable to livestock. This community type would be rated as primary range for domestic livestock. Wild sarsaparilla, a major component of this community type appears to be very sensitive to any disturbance by livestock.

\section{Plant Composition Canopy COVER(\%)}

TREES

MEAN RANGE CONST.

BALSAM POPLAR

(Populus balsamifera)

TREMBLING ASPEN

(Populus tremuloides) $\quad 52 \quad 10-75 \quad 100$

SHRUBS

RED OSIER DOGWOOD

(Cornus stolonifera)

WILD RED RASPBERRY

(Rubus ideaus)

PRICKLY ROSE

(Rosa acicularis)

LOW BUSH CRANBERRY

(Viburnum edule)

FORBS

WILD SARSAPARILLA

(Aralia nudicaulis)

YELLOW PEAVINE

(Lathyrus ochroleucus)

DEWBERRY

(Rubus pubescens)

LINDLEY'S ASTER

(Aster ciliolatus)

FIREWEED

(Epilobium angustifolium) 5

STRAWBERRY

(Fragaria virginiana)

GRASSES

MARSH REED GRASS

(Calamagrostis canadensis)7

$\begin{array}{lll}7 & 0-30 & 50 \\ 52 & 10-75 & 100\end{array}$

$\begin{array}{lll}3 & 0-7 & 59\end{array}$

$2 \quad 0-5 \quad 65$

$16 \quad 5-33 \quad 100$

$\begin{array}{lll}8 & 0-26 \quad 88\end{array}$

$20 \quad 6-69 \quad 100$

$1-12 \quad 100$

$0-15 \quad 88$

$0-2 \quad 81$

$0-19 \quad 81$

$0-9 \quad 81$

$2-21 \quad 100$

\section{ENVIRONMENTAL VARIABLES}

MOISTURE REGIME:

MESIC

NUTRIENT REGIME:

MESOTROPHIC TO PERMESOTROPHIC

ELEVATION:

617(576-697)M

SOIL DRAINAGE:

WELL

RANGELAND HEALTH RATING:

HEALTHY

\section{FORAGE PRODUCTION(KG/HA)}

$\begin{array}{ll}\text { GRASS } & 188(3-812) \\ \text { FORBS } & 379(179-480) \\ \text { SHRUBS } & 318(60-1058) \\ \text { TOTAL } & 884(459-1470)\end{array}$

ECOLOGICALLY SUSTAINABLE STOCKING RATE $2.0 \mathrm{HA} / \mathrm{AUM}(0.2 \mathrm{AUM} / \mathrm{AC})$ 


\section{CMC8a. Aw/Buffaloberry-Rose \\ (Populus tremuloides/Shepherdia canadensis-Rosa acicularis)}

$\mathbf{n = 1} \quad$ This community type was found on a mesic site at higher elevations in the Central Mixedwood subregion west of Beaverlodge. Beckingham (1993) felt the Aw/Buffaloberry type was slightly drier and had a slightly poorer nutrient regime than the modal Aw/Rose community types. This type is providing a moderate amount of forage for domestic livestock, but the drier site conditions and poorer nutrient status will limit regrowth after grazing. Buffaloberry the predominant shrub species in this community type, is generally unpalatable to livestock.

\section{Plant COMPOSITION CANOPY COVER(\%)}

MEAN RANGE CONST.

\section{TREES}

TREMBLING ASPEN

(Populus tremuloides)

$75 \quad-\quad 100$

\section{SHRUBS}

BUFFALOBERRY

(Shepherdia canadensis) $26 \quad-\quad 100$

PRICKLY ROSE

(Rosa acicularis)

LOW BUSH CRANBERRY

(Viburnum edule)

FORBS

BUNCHBERRY

(Cornus canadensis)

YELLOW PEAVINE

$\begin{array}{llll}\text { (Lathyrus ochroleucus) } & 2 & - & 100\end{array}$

DEWBERRY

(Rubus pubescens) $\quad 2 \quad-\quad 100$

FIREWEED

(Epilobium angustifolium) $6 \quad-\quad 100$

STRAWBERRY

(Fragaria virginiana) $\quad 3 \quad-\quad 100$

GRASSES

MARSH REED GRASS

(Calamagrostis canadensis)2 - $\quad 100$

\section{ENVIRONMENTAL VARIABLES}

MOISTURE REGIME:

MESIC

NUTRIENT REGIME:

MESOTROPHIC TO SUBMESOTROPHIC

ELEVATION:

$800 \mathrm{M}$

SOIL DRAINAGE:

WELL

RANGELAND HEALTH RATING:

HEALTHY

\section{FORAGE PRODUCTION(KG/HA)}

$\begin{array}{ll}\text { GRASS } & 61 \\ \text { FORBS } & 364 \\ \text { SHRUBS } & 532 \\ \text { TOTAL } & 957\end{array}$

ECOLOGICALLY SUSTAINABLE STOCKING RATE $2.0 \mathrm{HA} / \mathrm{AUM}(0.2 \mathrm{AUM} / \mathrm{AC})$ 


\section{CMC9. Pb-Aw/Rose-Saskatoon}

(Populus balsamifera-Populus tremuloides/Rosa acicularis-Amelanchier alnifolia)

$\mathbf{n}=\mathbf{2}$ This community type is found on mesic, well drained south facing slopes that overlook rivers and creeks.

This community is also similar to the Aw/Saskatoon-Rose community that was described in the Dry Mixedwood subregion. Both community types occur on south and west facing slopes. Saskatoon provides important browse for wild ungulates. Livestock also find saskatoon palatable and in areas where there is extensive cattle grazing this species can be heavily browsed.

\section{Plant COMPOSITION Canopy COVER(\%) MEAN RANGE CONST.}

\begin{tabular}{|c|c|c|c|}
\hline \\
\hline \multicolumn{4}{|l|}{ ASPEN } \\
\hline (Populus tremuloides) & 20 & $5-35$ & 100 \\
\hline $\begin{array}{l}\text { BALSAM POPLAR } \\
\text { (Populus balsamifera) }\end{array}$ & & & 100 \\
\hline $\begin{array}{l}\text { (Populus balsamifera) } \\
\text { SHRUBS }\end{array}$ & 44 & $7-80$ & 100 \\
\hline PRICKLY ROSE & & & \\
\hline (Rosa acicularis) & 22 & $6-38$ & 100 \\
\hline SASKATOON & & & \\
\hline (Amelanchier alnifolia) & 25 & $5-45$ & 100 \\
\hline RED OSIER DOGWOOD & & & \\
\hline (Cornus stolonifera) & 7 & $5-8$ & 100 \\
\hline RIVER ALDER & & & \\
\hline (Alnus tenuifolia) & 8 & $0-15$ & 50 \\
\hline WILLOW & & & \\
\hline (Salix spp.) & 8 & $0-15$ & 50 \\
\hline FORBS & & & \\
\hline HORSETAIL & & & \\
\hline (Equisetum arvense) & 3 & $1-3$ & 100 \\
\hline BUNCHBERRY & & & \\
\hline (Cornus canadensis) & 1 & $0-1$ & 50 \\
\hline TALL LUNGWORT & & & \\
\hline (Mertensia paniculata) & 2 & $0-3$ & 50 \\
\hline DEWBERRY & & & \\
\hline (Rubus pubescens) & 3 & $2-3$ & 100 \\
\hline YELLOW PEAVINE & & & \\
\hline (Lathyrus ochroleucus) & 4 & $0-8$ & 50 \\
\hline WILD SARSAPARILLA & & & \\
\hline (Aralia nudicaulis) & 3 & $0-5$ & 50 \\
\hline STRAWBERRY & & & \\
\hline (Fragaria virginiana) & 4 & $0-7$ & 50 \\
\hline GRASSES & & & \\
\hline MOUNTAIN RICEGRASS & & & \\
\hline (Oryzopsis asperifolia) & 1 & $0-1$ & 50 \\
\hline
\end{tabular}

\section{ENVIRONMENTAL VARIABLES}

MOISTURE REGIME:

MESIC

NUTRIENT REGIME: MESOTROPHIC

ELEVATION: $606 \mathrm{M}$

SOIL DRAINAGE:

WELL TO RAPIDLY

SLOPE:

$26(2-50) \%$

ASPECT:

WESTERLY

RANGELAND HEALTH RATING:

HEALTHY

\section{FORAGE PRODUCTION(KG/HA)}

$\begin{array}{ll}\text { GRASS } & 25(0-50) \\ \text { FORBS } & 279(240-318) \\ \text { SHRUBS } & 111(10-212) \\ \text { TOTAL } & 415(250-580)\end{array}$

ECOLOGICALLY SUSTAINABLE STOCKING RATE 4.0 HA/AUM (0.1 AUM/AC) 


\section{CMC10. Aw-Pb/Rose/Strawberry}

(Populus tremuloides-Populus balsamifera/Rosa acicularis/Fragaria virginiana)

$\mathbf{n}=\mathbf{4}$ This community type appears to have been moderately grazed in the past. As grazing pressure becomes heavy, there is a reduction in shrub, tall forbs and native grass cover and an increase in cover of low growing forbs(dandelion and strawberry). Continued heavy grazing pressure eventually leads to a decline in all native plants and Kentucky bluegrass, clover and dandelion will predominate in the understory (Willoughby 1996). The forage production on this community type is only moderate and is slightly less than other $\mathrm{Aw}$ and $\mathrm{Pb}$ dominated community types. A period of rest would greatly benefit the production on this community type.

\section{Plant COMPOSITION CANOPY COVER(\%)}

$$
\text { MEAN RANGE CONST. }
$$

\section{TREES}

WHITE BIRCH

$\begin{array}{lccc}\begin{array}{l}\text { (Betula papyrifera) } \\ \text { BALSAM POPLAR } \\ \text { (Populus balsamifera) }\end{array} & 3 & 0-10 & 25 \\ \begin{array}{l}\text { ASPEN } \\ \text { (Populus tremuloides) }\end{array} & 41 & 10-45 & 100 \\ \begin{array}{l}\text { SHRUBS } \\ \text { PRICKLY ROSE } \\ \text { (Rosa acicularis) }\end{array} & 14 & 6-25 & 100 \\ \begin{array}{l}\text { LOW BUSH CRANBERRY } \\ \text { Viburnum edule) }\end{array} & 1 & 0-5 & 25 \\ \begin{array}{l}\text { SNOWBERRY OR BUCKBRUSH } \\ \text { (Symphoricarpos }\end{array} & 6 & 1-14 & 100 \\ \begin{array}{l}\text { occidentalis) } \\ \text { WILD RED RASPBERRY } \\ \text { (Rubus idaeus) }\end{array} & 2 & 0-4 & 50\end{array}$

\section{FORBS}

WILD STRAWBERRY

(Fragaria virginiana) $13 \quad 1-30 \quad 100$

DANDELION

(Taraxacum officinale) $\quad 3 \quad 1-6 \quad 100$

DEWBERRY OR RUNNING RASPBERRY

$\begin{array}{llll}\text { (Rubus pubescens) } & 2 & 0-5 & 75\end{array}$

NORTHERN BEDSTRAW

(Galium boreale) $\quad 2 \quad 1-3 \quad 100$

LINDLEY'S ASTER

$\begin{array}{llll}\text { (Aster ciliolatus) } & 3 & 1-4 & 100\end{array}$

FIREWEED

(Epilobium angustifolium)2

GRASSES

MARSH REED GRASS

(Calamagrostis canadensis)7 $\quad 1-16 \quad 100$

\section{ENVIRONMENTAL VARIABLES}

MOISTURE REGIME:

MESIC TO SUBHYGRIC

NUTRIENT REGIME:

MESOTROPHIC

ELEVATION:

$$
\text { 576-606(584) M }
$$

PERCENT SLOPE GRADIENT:

LEVEL

SOIL DRAINAGE:

WELL TO MODERATELY WELL

RANGELAND HEALTH RATING:

HEALTHY WITH PROBLEMS

\section{FORAGE PRODUCTION(KG/HA)}

$\begin{array}{ll}\text { GRASS } & 259(0-617) \\ \text { FORBS } & 327(142-524) \\ \text { SHRUBS } & 128(23-234) \\ \text { TOTAL } & 709(496-916)\end{array}$

ECOLOGICALLY SUSTAINABLE STOCKING RATE $3.0 \mathrm{HA} / \mathrm{AUM}(0.15 \mathrm{AUM} / \mathrm{AC})$ 


\section{CMC11. Aw/Rose/Clover \\ (Populus tremuloides/Rosa acicularis/Trifolium spp.)}

$\mathbf{n = 1}$ This community type is represented by aspen stands that have recieved moderate to heavy grazing pressure for a number of years. As a result, native forbs have declined and clover has increased in the understory. A small portion of the original shrub and tall forb understory still remains. Although, grass production has dropped, forb production remains high due to the dense cover of clover. This community has not been grazed as long as the Aw/Kentucky bluegrass/Clover community type (Willoughby 1996).

\section{PLANT COMPOSITION CANOPY COVER(\%)} MEAN RANGE CONST.

\section{TREES}

TREMBLING ASPEN

(Populus tremuloides)

BALSAM POPLAR

(Populus balsamifera)

SHRUBS

WILD RED RASPBERRY

(Rubus idaeus)

PRICKLY ROSE

(Rosa acicularis)

FORBS

ClOVER

(Trifolium sp.)

DANDELION

(Taraxacum officinale) $4 \quad$ - $\quad 100$

WILD STRAWBERRY

(Fragaria virginiana)

HORSETAIL

$\begin{array}{llll}\text { (Equisetum arvense) } & 4 & - & 100\end{array}$

WILD LILY-OF-THE-VALLEY

(Maianthemum canadense) 1

GRASSES

MARSH REED GRASS

(Calamagrostis canadensis)2 - 100

KENTUCKY BLUEGRASS

(Poa pratensis)

CREEPING RED FESCUE

(Festuca rubra)
30

18

3

5

18

1

100

$-100$

$10 \quad-\quad 100$

$5 \quad-\quad 100$

\section{ENVIRONMENTAL VARIABLES}

MOISTURE REGIME:

MESIC

NUTRIENT REGIME:

MESOTROPHIC

ELEVATION:

$606 \mathrm{M}$

SOIL DRAINAGE:

WELL

RANGELAND HEALTH RATING:

UNHEALTHY

\section{FORAGE PRODUCTION(KG/HA)}

$\begin{array}{ll}\text { GRASS } & 512 \\ \text { FORBS } & 192 \\ \text { SHRUBS } & 224 \\ \text { TOTAL } & 928\end{array}$

ECOLOGICALLY SUSTAINABLE STOCKING RATE $2.0 \mathrm{HA} / \mathrm{AUM}(0.2 \mathrm{AUM} / \mathrm{AC})$ 


\section{CMC12. Aw/Alder-Willow-Rose \\ (Populus tremuloides/Alnus crispa-Salix spp.-Rosa acicularis)}

$\mathbf{n = 1 3}$ This community type is scattered throughout the Central Mixedwood subregion on mainly mesic to subhygric, well-drained sites. This community is likely of fire origin. Many of the plots were described from a large fire that burned through the area in 1968. The aspen trees are also young and very dense. The high cover of aspen. alder, and willow limits the amount of light reaching the understory. Consequently, there is little forage available for domestic livestock. This community type would be rated as secondary or non-use range.

\section{PLANT COMPOSITION CANOPY COVER(\%)}

MEAN RANGE CONST.

\section{TREES}

TREMBLING ASPEN

(Populus tremuloides) $\quad 59 \quad 30-90 \quad 100$

SHRUBS

PRICKLY ROSE

(Rosa acicularis) $11 \quad 0-23 \quad 92$

BLUEBERRY

(Vaccinium myrtilloides)

WILLOW

(Salix spp.)

GREEN ALDER

(Alnus crispa)

FORBS

BUNCHBERRY

(Cornus canadensis)

WILD STRAWBERRY

(Fragaria virginiana)

TWINFLOWER

(Linnaea borealis)

DEWBERRY

(Rubus pubescens) 4

WILD LILY-OF-THE-VALLEY

(Maianthemum canadense)2

YELLOW PEAVINE

(Lathyrus ochroleucus) 2

GRASSES

MARSH REED GRASS

(Calamagrostis canadensis) $5 \quad 0-19 \quad 92$

HAIRY WILDRYE

$\begin{array}{llll}\text { (Elymus innovatus) } & 1 & 0-2 & 39\end{array}$

MOUNTAIN RICEGRASS

(Oryzopsis asperfolia)

$12 \quad 0-43 \quad 61$

$31 \quad 0-85 \quad 92$

$10 \quad 0-26 \quad 92$

$2 \quad 0-6 \quad 61$

$11 \quad 0-45 \quad 85$

$0-10 \quad 85$

$0-6 \quad 77$

$0-9 \quad 69$

$\begin{array}{lll}\mathrm{T} & 0-2 & 15\end{array}$

\section{ENVIRONMENTAL VARIABLES}

MOISTURE REGIME:

MESIC TO SUBHYGRIC

NUTRIENT REGIME:

MESOTROPHIC TO PERMESOTROPHIC

ELEVATION:

$635(333-758) \mathrm{M}$

PERCENT SLOPE GRADIENT:

$5(0-15) \%$

SOIL DRAINAGE:

WELL TO MODERATELY WELL

RANGELAND HEALTH RATING:

HEALTHY

FORAGE PRODUCTION(KG/HA)

GRASS 75(8-350)

FORBS 200(2-476)

SHRUBS 225(5-660)

TOTAL 499(100-930)

ECOLOGICALLY SUSTAINABLE STOCKING RATE $3.6 \mathrm{HA} / \mathrm{AUM}(0.1 \mathrm{AUM} / \mathrm{AC})$ 


\section{CMC13. Aw/Willow \\ (Populus tremuloides/Salix spp.)}

$\mathrm{n}=4$ This community type is similar to the Aw/Alder-Willow-Rose community type, but lacks the cover of alder. Previously, this community type was split into four community types (Willoughby and Downing 1995). These included the Aw/Willow-Rose/Twinflower, Aw/Willow-Rose/Bunchberry, Aw/Rose-Willow-Pin cherry/Fireweed and Aw/Rose-Willow-Saskatoon. All four community types appeared to have had a fire origin, but had slightly different moisture and nutrient regimes which affected forage productivity. Productivity varied from 1326 to $1306 \mathrm{~kg} / \mathrm{ha}$ on the Aw/Willow-Rose/Bunchberry and Aw/Rose-Willow-Pin cherry/Fireweed types to $606 \mathrm{~kg} / \mathrm{ha}$ on the Aw/Willow-Rose/Twinflower type. Because the sample size was so small it was felt to be impractical to split the four community types and they were lumped into this one type for the purpose of this guide. As this community undergoes succession forage productivity will decline.

\section{Plant COMPOSITION CANOPYCOVER(\%) \\ MEAN RANGE CONST.}

TREES

TREMBLING ASPEN

(Populus tremuloides) $53 \quad 40-68 \quad 100$

SHRUBS

PRICKLY ROSE

(Rosa acicularis)

WILLOW SPP.

(Salix spp.)

LOW BUSH CRANBERRY

(Viburnum edule)

FORBS

BUNCHBERRY

(Cornus canadensis)

(Aralia nudicaulis)

YELLOW PEAVINE

(Lathyrus ochroleucus)

DEWBERRY

(Rubus pubescens) 7
WILD LILY-OF-THE-VALLEY

(Maianthemum canadense)6

FIREWEED

(Epilobium angustifolium) $11 \quad 3-32 \quad 100$

TWINFLOWER

(Linnaea borealis) $\quad 5 \quad 1-11 \quad 100$

GRASSES

MARSH REED GRASS

(Calamagrostis canadensis)

HAIRY WILDRYE

(Elymus innovatus)
$13 \quad 11-15 \quad 100$

$27 \quad 15-35 \quad 100$

0-12 75

$13 \quad 2-30 \quad 100$

$9 \quad 0-18 \quad 75$

$1-10 \quad 100$

2-12 100

3-11 100

$1-13 \quad 100$

$1-10 \quad 100$

\section{ENVIRONMENTAL VARIABLES}

MOISTURE REGIME:

MESIC TO SUBHYGRIC

NUTRIENT REGIME:

MESOTROPHIC TO PERMESOTROPHIC

ELEVATION:

542(333-636) M

SOIL DRAINAGE:

WELL TO MODERATELY WELL

RANGELAND HEALTH RATING:

HEALTHY

\section{FORAGE PRODUCTION(KG/HA)}

GRASS 461(2-708)

FORBS 493(362-552)

SHRUBS 209(107-378)

TOTAL 1162(606-1367)

ECOLOGICALLY SUSTAINABLE STOCKING RATE $1.6 \mathrm{HA} / \mathrm{AUM}(0.25 \mathrm{AUM} / \mathrm{AC})$ 


\section{CMC14. Aw-Pb/Red osier dogwood-Rose \\ (Populus tremuloides-P. balsamifera/Cornus stolonifera-Rosa acicularis)}

$\mathbf{n}=\mathbf{8}$ This community is typical of river floodplains throughout the Central Mixedwood subregion. This community has a subhygric moisture and rich nutrient regime. Beckingham and Archibald (1996) found this community type on mid to lower slope topographic positions or near water courses where they recieve nutrient-rich seepage or flood waters for a portion of the growing season. This community type is one of the most productive in the Central Mixedwood, but the high cover of shrubs limits access to livestock. The high cover of tall growing shrubs (alder, red osier dogwood) also limits the growth of low shrubs, forbs and grass the principle forage species for domestic livestock in deciduous forests. As a result, this community should be rated as secondary or non-use range.

\section{Plant COMPOSITION CANOPY COVER(\%)}

MEAN RANGE CONST.

\section{TREeS}

TREMBLING ASPEN

(Populus tremuloides)

BALSAM POPLAR

(Populus balsamifera)

$53 \quad 25-70 \quad 100$

SHRUBS

PRICKLY ROSE

(Rosa acicularis)

RED OSIER DOGWOOD

(Cornus stolonifera)

RIVER ALDER

(Alnus tenuifolia)

FORBS

LADY FERN

(Athyrium filix-femina)

WILD SARSAPARILLA

(Aralia nudicaulis)

DEWBERRY

(Rubus pubescens)

WILD LILY-OF-THE-VALLEY

(Maianthemum canadense) 1

YELLOW PEAVINE

(Lathyrus ochroleucus) 4

GRASSES

MARSH REED GRASS

(Calamagrostis canadensis)

HAIRY WILDRYE

(Elymus innovatus)

$14 \quad 0-25 \quad 75$

$\begin{array}{lll}8 & 0-26 \quad 88\end{array}$

$22 \quad 3-40 \quad 100$

$3 \quad 0-5 \quad 38$

$1 \quad 0-11 \quad 25$

$3 \quad 0-10 \quad 75$

$4 \quad 0-6 \quad 63$

$0-10 \quad 50$

$1-10 \quad 100$

$0-1 \quad 38$
$1-10 \quad 100$
ENVIRONMENTAL VARIABLES

MOISTURE REGIME:

SUBHYGRIC

NUTRIENT REGIME:

PERMESOTROPHIC

ELEVATION:

602(600-606)M

SLOPE PERCENT:

$2(1-3) \%$

SOIL DRAINAGE:

MODERATELY WELL

RANGELAND HEALTH RATING:

HEALTHY

\section{FORAGE PRODUCTION(KG/HA)}

GRASS $\quad 7(2-22)$

FORBS 205(66-372)

SHRUBS 265(20-358)

TOTAL 476(226-714)

ECOLOGICALLY SUSTAINABLE STOCKING RATE NON-USE 


\section{CMC15. Aw/Horsetail-Cow parsnip \\ (Populus tremuloides/Equisetum arvense-Heracleum lanatum)}

$\mathbf{n = 1}$ This community type occupies lowland sites adjacent to black spruce and willow lowlands. It is very moist and nutrient rich. Horsetail types in other subregions also tend to be moister and richer than the modal Aw/Rose types. This site is very productive and produces a large amount of forage for domestic livestock. Horsetail is generally unpalatable to livestock and can be poisonous to horses. In contrast cow parsnip is very palatable to livestock. This community type would therefore be rated as primary or secondary range for domestic livestock.

\section{Plant COMPOSITION CANOPY COVER(\%)}

\section{TREES}

\section{MEAN RANGE CONST.}

\section{TREMBLING ASPEN}

(Populus tremuloides)

SHRUBS

WILD RED RASPBERRY

(Rubus idaeus)

FORBS

COW PARSNIP

(Heracleum lanatum) 30

FIREWEED

(Epilobium angustifolium) 1

HORSETAIL

TALL LUNGWORT

$\begin{array}{llll}\text { (Mertensia paniculata) } & 18 & - & 100\end{array}$

TALL LARKSPUR

(Delphinium glaucum) $13 \quad$ - 100

GRASSES

MARSH REED GRASS

(Calamagrostis canadensis) 47

\section{ENVIRONMENTAL VARIABLES}

MOISTURE REGIME:

SUBHYGRIC TO HYGRIC

NUTRIENT REGIME:

PERMESOTROPHIC

ELEVATION:

$758 \mathrm{M}$

SOIL DRAINAGE:

MODERATELY WELL

RANGELAND HEALTH RATING:

HEALTHY

\section{FORAGE PRODUCTION(KG/HA)}

$\begin{array}{ll}\text { GRASS } & 1292 \\ \text { FORBS } & 1440 \\ \text { TOTAL } & 2732\end{array}$

ECOLOGICALLY SUSTAINABLE STOCKING RATE

$0.7 \mathrm{HA} / \mathrm{AUM}(0.55 \mathrm{AUM} / \mathrm{AC})$ 


\section{CMC16. Aspen/ Smooth brome \\ (Populus tremuloides/Bromus inermis)}

$\mathbf{n}=\mathbf{1}$ This community type is similar to the previously described red osier dogwood dominated community type, but has a high cover of smooth brome in the understory. Smooth brome is an introduced grass that can increase with increased grazing pressure, but smooth brome is also highly invasive and can invade into ungrazed areas. The invasion of non-native invaders onto the site makes this community moderately productive for domestic livestock.

Plant Composition Canopy COVER(\%) Mean Range Const.

\section{TREES}

ASPEN

(Populus tremuloides)

80

100

SHRUBS

ROSE

(Rosa acicularis)

SNOWBERRY

(Symphoricarpos

occidentalis)

RED OSIER DOGWOOD

(Cornus stolonifera)

FORBS

TALL LUNGWORT

(Mertensia paniculata)

VEINY MEADOW RUE

(Thalictrum venulosum)

YELLOW PEAVINE

(Lathyrus ochroleucus)

\section{Grasses}

SMOOTH BROME

(Bromus inermis)

MARSH REEDGRASS

(Calamagrostis canadensis) 3

\section{ENVIRONMENTAL VARIABLES}

MoIsTURE REgIME:

MESIC-SUBHYGRIC

NUTRIENT REGIME:

MESOTROPHIC-PERMESOTROPHIC

ELEVATION:

$600 \mathrm{M}$

SOIL DRAINAGE:

MODERATELY WELL

HEALTH RATING:

HEALTHY WITH PROBLEMS

FORAGE PRODUCTION(KG/HA)

TOTAL: $\quad 1100 *$ Estimate

ECOLOGICALLY SUSTAINABLE STOCKING RATE

$1.8 \mathrm{HA} / \operatorname{AUM}(0.2 \mathrm{AUM} / \mathrm{AC})$ 


\section{CENTRAL MIXEDWOOD SUBREGION}

\section{CONIFEROUS AND MIXEDWOOD FOREST COMMUNITIES}

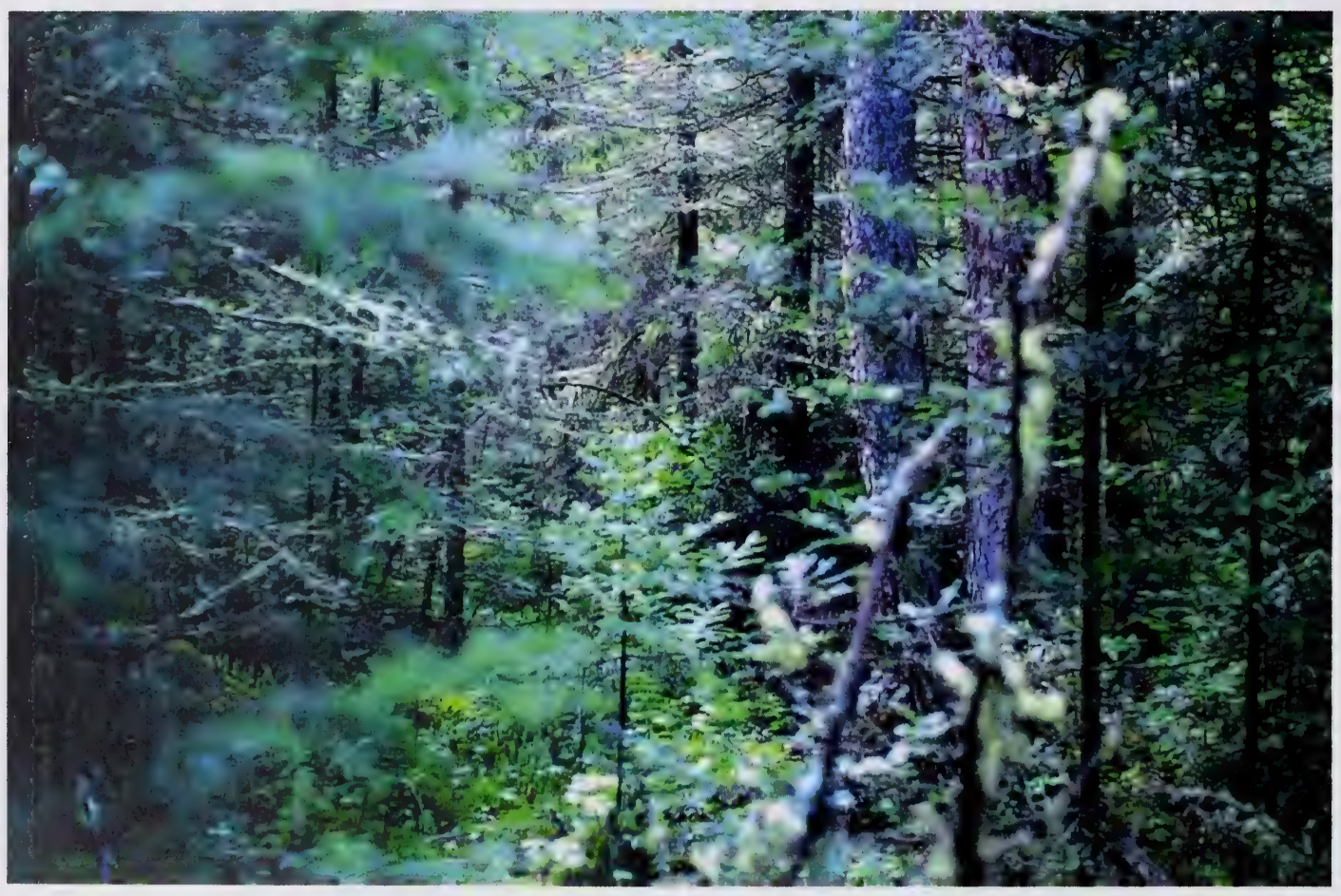

Photo 10. The Balsam fir-White spruce/Moss community type is the climatic climax community for the Central Mixedwood subregion. 


\section{CM-CONIFEROUS AND MIXEDWOOD FORESTS}

The mixedwood and coniferous community types described in this guide represent five ecological sites as described by Beckingham and Archibald (1996). On sites with subxeric moisture and poor nutrient regimes, coarse textured, sandy soils open stands of jack pine generally dominate $(\mathrm{Pj} / \mathrm{Alder}, \mathrm{Pj} /$ Bearberry). These community types commonly have a carpet of lichens covering the forest floor and a thin organic layer typically less than $5 \mathrm{~cm}$ thick (Beckingham and Archibald 1996).

On slightly moister sites with submesic moisture and medium nutrient regimes aspen grows in conjunction with jack pine to form the $\mathrm{Aw}-\mathrm{Pj} / \mathrm{Bearberry} / \mathrm{Lichen}$ community type. The soils of this community type continue to be coarse-textured but the moisture and nutrient conditions are more favourable to the growth of aspen.

The mesic/medium sites are generally dominated by white spruce (Balsam fir-Sw/Moss, $\mathrm{Sw} / \mathrm{Moss}, \mathrm{Sw} / \mathrm{Creeping}$ red fescue) and mixedwood communities of aspen and spruce (Aw$\mathrm{Sw} / \mathrm{Rose} / \mathrm{Low}$ forb). These communities represent the reference ecosite for the Boreal Mixedwood subregion (Beckingham and Archibald 1996). Generally, these sites have moderately fine to fine-textured till or glaciolacustrine parent materials. Pioneer deciduous species (aspen, balsam poplar and birch) are replaced with white spruce and balsam fir as these sites develop successionally. With succession shade tolerant plants take over the herbaceous layer as conifers dominate the canopy. These shade tolerant species are unproductive and often unpalatable for domestic livestock. Forage productivity declines from $2.3 \mathrm{ha} / \mathrm{AUM}$ in a deciduous community to 2.3-8.6 ha/AUM in a mixedwood community to less than $10 \mathrm{ha} / \mathrm{AUM}$ in a conifer community.

Black spruce and larch communites generally dominate on wetter sites with subhygric to subhydric moisture regimes and poor to medium nutrient regimes to form the $\mathrm{Sb} / \mathrm{Bog}$ birch and $\mathrm{Sb} / \mathrm{Labrador}$ tea/Moss community types. Larch is more tolerant of excessive moisture and is indicative of an enriched nutrient status, while black spruce is typical in areas of stagnating ground water with poor nutrient status (Hay et al. 1985). Generally, these community types are considered non-use for domestic livestock.

Beckingham and Archibald (1996), provide a good description on how the conifer and mixedwood communities are arranged in the landscape. 


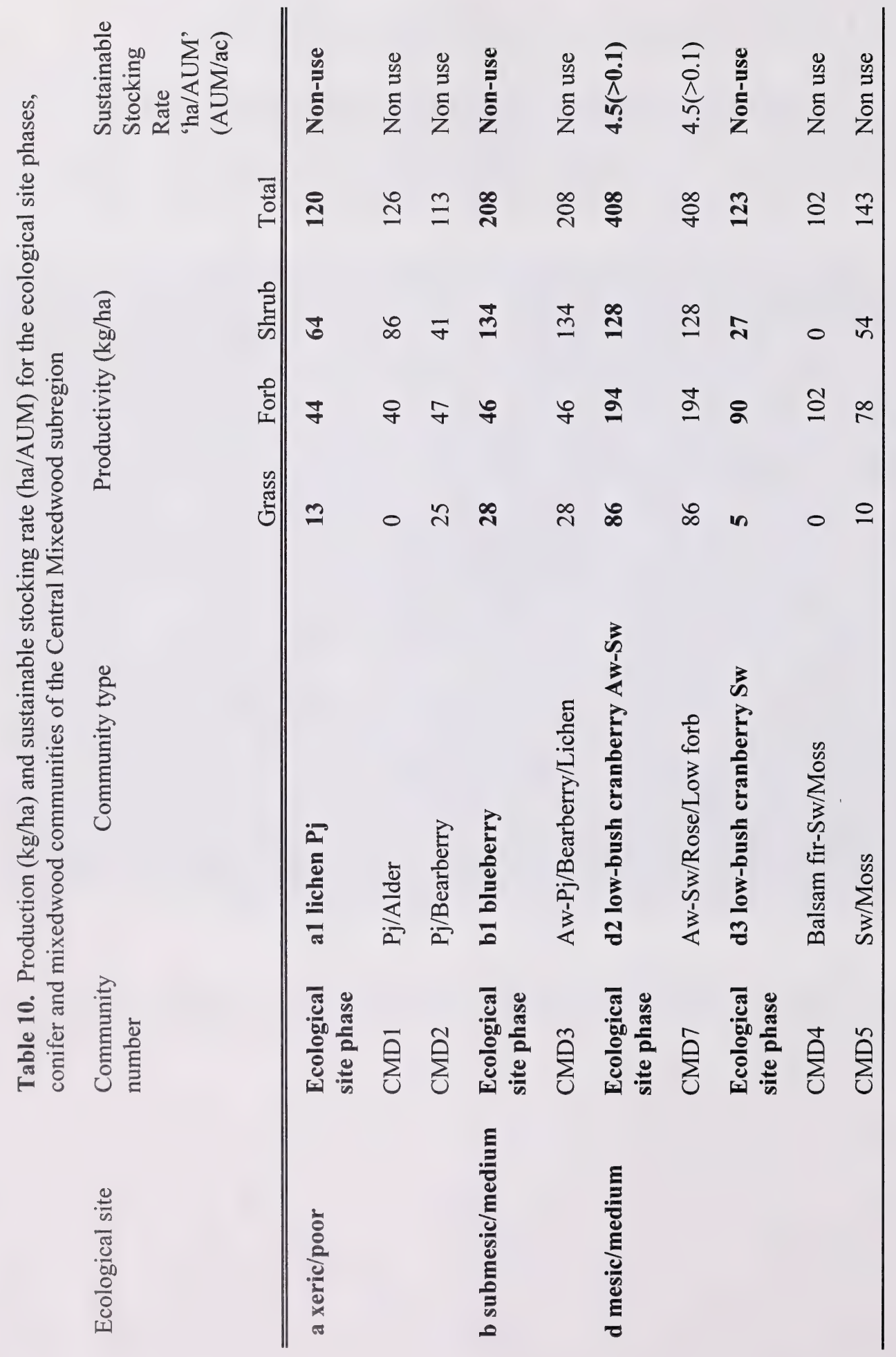

$\stackrel{\infty}{N}$ 


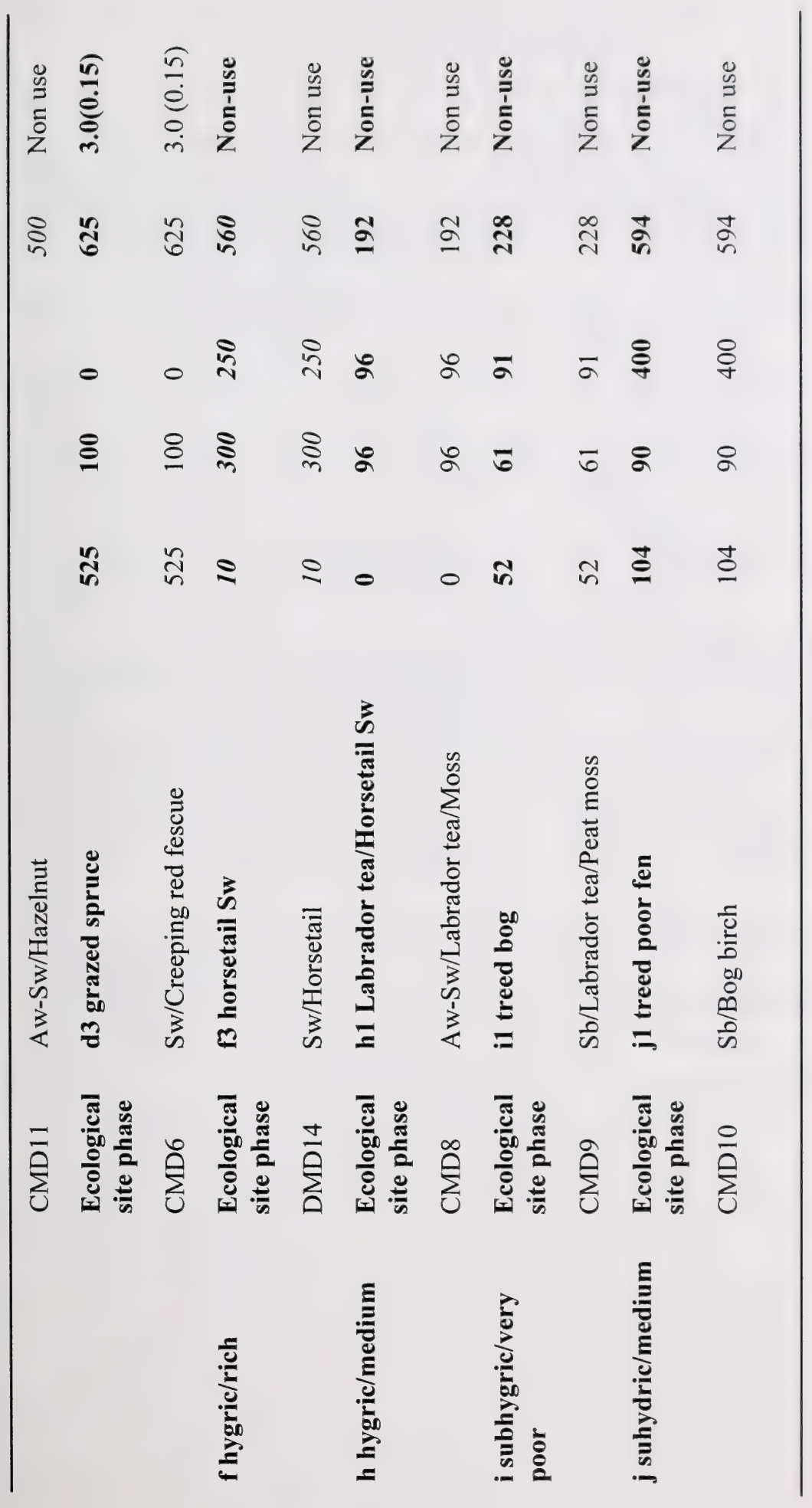




\section{Conifer and Mixedwood Types - Central Mixedwood Subregion}

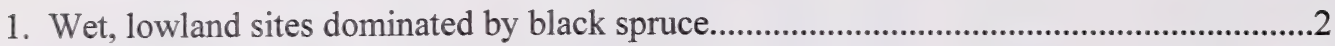

Upland mesic sites or well drained sandy sites.....................................................................

2. Bog birch, sedge dominate understory(rich fen).

$. \mathrm{Sb} / \operatorname{Bog} \operatorname{Birch}(\mathrm{CMD} 10)$

Labrador tea dominates understory, larch

present (poor fen)

Sb/Labrador Tea/Peat Moss (CMD9)

3. Mesic sites dominated by spruce, aspen, balsam poplar (maybe co-dominated by jack pine).

Dry, sandy sites dominated by jack pine.........................................................................

4. Mixedwood types, mixture of conifer and deciduous trees................................................

White spruce or balsam fir dominated types.......................................................................

5. Aw-Sw mixedwood, typical mesic sites

.6

Drier sites with Jack pine, Aw-Pj dominated

Aw-Pj/Bearberry/Lichen (CMD3)

6. Rose, low forb, hazelnut dominated, typical mesic sites

$6 a$

Labrador tea dominated, poorer nutrient sites.

Aw-Sw/Labrador Tea/Moss (CMD8)

6a. Rose, low forb dominated Aw-Sw/Rose/Low Forb (CMD7) Hazelnut dominated. Aw-Sw/Hazelnut (CMD11)

7. Balsam fir dominates(old growth forest) Balsam Fir-Sw/Moss (CMD4) White spruce dominates overstory....... $.7 \mathrm{a}$

7a. Moss dominates understory. .Sw/Moss (CMD5) Horsetail dominates understory Sw/Horsetail(CMD12)

8. Jack pine overstory, bearberry or lichen dominates understory, alder low in cover or absent. $\mathrm{Pj} /$ Bearberry (CMD2) Jack pine overstory, alder dominates understory Pj/Alder (CMD1) 


\section{CMD1. Pj/Alder \\ (Pinus banksianal Alnus crispa)}

$\mathbf{n}=\mathbf{1}$ This community type is found on dry, rapidly drained, sandy soils with a poor nutrient status. Consequently, production is quite low. Cattle will utilize these areas due to the easy access, however overutilization will quickly deplete the area of forage. This community type would be rated as secondary or non-use range.

\section{Plant Composition Canopy Cover(\%) MEAN RANGE CONST.}

TREES

JACK PINE

(Pinus banksiana)

$$
45
$$

SHRUBS

GREEN ALDER

(Alnus crispa)

PRICKLY ROSE

(Rosa acicularis)

BLUEBERRY

(Vaccinium myrtilloides) $13 \quad-\quad 100$

FORBS

TWIN-FLOWER

(Linnaea borealis) $\quad 4 \quad$ - $\quad 100$

BEARBERRY

(Arctostaphylos uva-ursi) T $\quad$ - $\quad 100$

WILD SARSAPARILLA

$\begin{array}{lll}\text { (Aralia nudicaulis) } & 3 & -\end{array}$

WILD LILY-OF-THE-VALLEY

(Maianthemum canadense) 4

GRASSES

SEDGES

(Carex spp.)

HAIRY WILD RYE

(Elymus innovatus)

NORTHERN RICEGRASS

(Oryzopsis pungens)

\section{ENVIRONMENTAL VARIABLES}

MOISTURE REGIME:

SUBXERIC

NUTRIENT REGIME

POOR

ELEVATION:

$606 \mathrm{M}$

SOIL DRAINAGE:

RAPIDLY

PERCENT SLOPE GRADIENT:

$$
2-8
$$

RANGELAND HEALTH RATING:

HEALTHY

\section{FORAGE PRODUCTION (KG/HA)}

$\begin{array}{ll}\text { GRASS } & 0 \\ \text { FORBS } & 40 \\ \text { SHRUBS } & 86 \\ \text { TOTAL } & 126\end{array}$

ECOLOGICALLY SUSTAINABLE STOCKING RATE NON-USE 


\section{CMD2. Pj/Bearberry \\ (Pinus banksiana/Arctostaphylos uva-ursi)}

$\mathbf{n}=\mathbf{2}$ This community represents a jack pine forest which is very similar to the Pj/Alder community type. Like the previous community cattle will utilize these areas due to the easy access, however overutilization will quickly deplete the forage supply. This community type would be rated as secondary range and should be grazed on a single rotation per year.

Plant COMPOSITION CANOPY COVER(\%) MEAN RANGE CONST.

TREES

JACK PINE

(Pinus banksiana) $\quad 38 \quad 30-45 \quad 100$

ASPEN

(Populus tremuloides) $\quad \mathrm{T} \quad 0-1 \quad 50$

\section{SHRUBS}

BOG CRANBERRY

(Vaccinium vitis-idaea) $\quad 6 \quad 0-11 \quad 50$

PRICKLY ROSE

(Rosa acicularis) $\quad \mathrm{T} \quad 0-1 \quad 50$

BLUEBERRY

(Vaccinium myrtilloides) $2 \quad 0-3 \quad 50$

FORBS

BEARBERRY

(Arctostaphylos uva-ursi) $18 \quad 16-19 \quad 100$

NORTHERN BEDSTRAW

(Galium boreale) T

(Maianthemum canadense) 1

PHILADEPHLIA FLEABANE

(Erigeron philadelphicus) 1

GRASSES

HAIRY WILD RYE

(Elymus innovatus)

SEDGE SPP.

Carex spp.)

NORTHERN RICEGRASS

(Oryzopsis pungens)

Mosses

Moss spp.
T $\quad 0-1 \quad 50$

$0-1 \quad 100$

$0-1 \quad 50$

$2 \quad 0-3 \quad 50$

$6 \quad 0-11 \quad 100$

$2 \quad 1-2 \quad 100$

$18 \quad 0-35 \quad 100$

\section{ENVIRONMENTAL VARIABLES}

MOISTURE REGIME:

SUBMESIC

NUTRIENT REGIME:

SUBMESOTROPHIC

ELEVATION:

624(576-671) M

SOIL DRAINAGE:

RAPIDLY

PERCENT SLOPE GRADIENT:

$10 \%$

RANGELAND HEALTH RATING:

HEALTHY

FORAGE PRODUCTION (KG/HA)

$\begin{array}{ll}\text { GRASS } & 25(0-50) \\ \text { FORBS } & 47(40-54) \\ \text { SHRUBS } & 41(10-72) \\ \text { TOTAL } & 113(100-126)\end{array}$

ECOLOGICALLY SUSTAINABLE STOCKING RATE

NON-USE 


\section{CMD3. Aw-Pj/Bearberry/Lichen \\ (Populus tremuloides-Pinus banksiana/Arctostaphylos uva-ursi/Lichen)}

$\mathbf{n}=\mathbf{2}$ This community type represents a aspen forest with a secondary canopy of jack pine. It is very similar to the $\mathrm{Pj} /$ Bearberry community type, but it is found on slightly moister soils with better nutrients. These conditions favour the growth of aspen. Like the previous community cattle will utilize these areas due to the easy access, however overutilization will quickly deplete the forage supply. This community type would be rated as secondary range and should be grazed on a single rotation per year.

\section{Plant COMPOSITION CANOPY COVER(\%) \\ MEAN RANGE CONST.}

\section{TREES}

JACK PINE

(Pinus banksiana)

$15 \quad 10-20 \quad 100$

ASPEN

(Populus tremuloides)

$20 \quad 15-25 \quad 100$

SHRUBS

BOG CRANBERRY

(Vaccinium vitis-idaea) $\quad 4 \quad 0-8 \quad 50$

PRICKLY ROSE

(Rosa acicularis) 1

BLUEBERRY

(Vaccinium myrtilloides) 8

FORBS

BEARBERRY

(Arctostaphylos uva-ursi) 8

TWINFLOWER

(Linnaea borealis) T

WILD LILY-OF-THE-VALLEY

(Maianthemum canadense)2

TOADFLAX

(Comandra umbellata) 1

GRASSES

SLENDER WHEATGRASS

(Agropyron trachycaulum) $2 \quad 0-4 \quad 50$

NORTHERN RICEGRASS

(Oryzopsis pungens)

SEDGE

(Carex spp.)

LICHENS

\section{$0-1 \quad 50$}

$0-15 \quad 50$

2-12 $\quad 100$

$0-1 \quad 50$

$0-3 \quad 50$

$0-1 \quad 100$

$2 \quad 0-4 \quad 50$

$\begin{array}{lll}4 & 0-7 & 100\end{array}$

$49 \quad 16-81 \quad 100$

\section{ENVIRONMENTAL VARIABLES}

MOISTURE REGIME:

SUBMESIC

NUTRIENT REGIME:

SUBMESOTROPHIC

ELEVATION:

$576 \mathrm{M}$

SOIL DRAINAGE:

WELL

RANGELAND HEALTH RATING:

HEALTHY

Forage Production (KG/Ha)

GRASS $\quad 28$

FORBS $\quad 46$

SHRUBS $\quad 134$

TOTAL 208

ECOLOGICALLY SUSTAINABLE STOCKING RATE NON-USE 


\section{CMD4. Balsam fir-Sw/Moss \\ (Abies balsamea-Picea glauca/Moss)}

$\mathbf{n}=\mathbf{1}$ This is a mature balsam fir forest which represents the climax vegetation for the area. The northerly aspect of this community type has probably protected the site from past disturbance by fires and allowed the community to undergo succession. The high canopy of balsam fir and spruce limits the light reaching the forest floor, limiting the growth of grasses and forbs. As a result, the forage productivity of this community type is very low. This community would be considered non-use.

\section{Plant COMPOSITION CANOPY COVER(\%)}

\section{MEAN RANGE CONST.}

TREeS

WHITE SPRUCE

(Picea glauca)

BALSAM FIR

(Abies balsamea)

\section{SHRUBS}

PRICKLY ROSE

(Rosa acicularis)

FORBS

BUNCHBERRY

(Cornus canadensis)

TWINFLOWER

(Linnaea borealis)

WOODLAND HORSETAIL

(Equisetum sylvaticum)

RUNNING CLUBMOSS

(Lycopodium clavatum)

$\begin{array}{lll}25 & - & 100 \\ 40 & - & 100 \\ \mathrm{~T} & - & 100\end{array}$

10

100

100

100

MOSSES

FEATHER MOSS

$\begin{array}{llll}\text { (Pleurozium schreberi) } & 51 & - & 100\end{array}$

STAIRSTEP MOSS

(Hylocomium splendens)

\section{ENVIRONMENTAL VARIABLES}

MOISTURE REGIME:

MESIC

NUTRIENT REGIME:

MESOTROPHIC

ELEVATION:

$333 \mathrm{M}$

SOIL DRAINAGE:

WELL

PERCENT SLOPE GRADIENT:

$5 \%$

ASPECT:

NORTHERLY

RANGELAND HEALTH RATING:

HEALTHY

FORAGE PRODUCTION (KG/HA)

GRASS $\quad 0$

FORBS $\quad 102$

SHRUBS 0

TOTAL $\quad 102$

ECOLOGICALLY SUSTAINABLE STOCKING RATE NON-USE 


\section{CMD5. Sw/Moss \\ (Picea glauca/Moss)}

$\mathbf{n}=\mathbf{8}$ This community is considered successionally mature. A more continuous cover of feather moss and presence of balsam fir would bring this community type closer to the climax community described previously. The limited light penetration in this community discourages understory development, making this a non-use area for domestic livestock.

\section{Plant COMPOSITION CANOPY COVER(\%)} MEAN RANGE CONST.

\section{TREES}

WHITE SPRUCE

(Picea glauca)

ASPEN

(Populus tremuloides)

SHRUBS

PRICKLY ROSE

(Rosa acicularis)

RED OSIER DOGWOOD

(Cornus stolonifera)

LOW BUSH CRANBERRY

(Viburnum edule)

FORBS

BUNCHBERRY

$\begin{array}{llll}\text { (Cornus canadensis) } & 7 & 2-14 & 86\end{array}$

FIELD HORSETAIL

$\begin{array}{llll}\text { (Equisetum arvense) } & 1 & 0-3 & 29\end{array}$

TWINFLOWER

(Linnaea borealis) 7

(Petasites palmatus) $3 \quad 0-5 \quad 85$

DEWBERRY

(Rubus pubescens) $\quad 1 \quad 0-3 \quad 57$

FIREWEED

(Epilobium angustifolium) 1

GRASSES

MARSH REEDGRASS

(Calamagrostis canadensis) $1 \quad 0-2 \quad 71$

Moss

STAIR STEP MOSS

(Hylocomium splendens) $13 \quad 0-49 \quad 19$

FEATHERMOSS

$\begin{array}{llll}\text { (Pleurozium schreberi) } & 1 & 0-7 & 17\end{array}$

\section{ENVIRONMENTAL VARIABLES}

MOISTURE REGIME:

MESIC

NUTRIENT REGIME:

MESOTROPHIC

ELEVATION:

415(150-606) M

SOIL DRAINAGE:

WELL

PERCENT SLOPE GRADIENT:

$1 \%$

RANGELAND HEALTH RATING:

HEALTHY

Forage Production(KG/Ha)

$\begin{array}{ll}\text { GRASS } & 10(0-40) \\ \text { FORBS } & 78(0-172) \\ \text { SHRUBS } & 54(0-158) \\ \text { TOTAL } & 143(36-370)\end{array}$

ECOLOGICALLY SUSTAINABLE STOCKING RATE NON-USE 


\section{CMD6. Sw/Creeping red fescue \\ (Picea glauca/Festuca rubra)}

$\mathbf{n}=\mathbf{1}$ This community type represents an old cultivated field which has been planted to white spruce. The canopy of spruce is beginning to shade the understory causing a decline in productivity, however, there is still enough forage for grazing between the spruce trees.

\section{Plant COMPOSITION CANOPY COVER(\%) MEAN RANGE CONST.}

TREES

ASPEN

(Populus tremuloides) $\quad 1 \quad-\quad 100$

WHITE SPRUCE

(Picea glauca)

BALSAM POPLAR

$\begin{array}{llll}\text { (Populus balsamifera) } & 1 & - & 100\end{array}$

SHRUBS

SNOWBERRY

(Symphoricarpos occidentalis)5 - 100

PRICKLY ROSE

(Rosa acicularis)

FORBS

STRAWBERRY

(Fragaria virginiana) $11 \quad-\quad 100$

CLOVER

(Trifolum hybridum) $\quad 5 \quad-\quad 100$

DANDELION

$\begin{array}{llll}\text { (Taraxacum officinale) } & 5 & - & 100\end{array}$

LINDLEY'S ASTER

(Aster ciliolatus)

GRASSES

CREEPING RED FESCUE

(Festuca rubra)

HAIRY WILDRYE

(Elymus innovatus)

SLENDER WHEATGRASS

(Agropyron trachycaulum) 1

SEDGE

(Carex spp.)
$10 \quad-\quad 100$

(1)

00

100

$3 \quad-\quad 100$

$29 \quad-\quad 100$

$12 \quad-100$

100

100

\section{ENVIRONMENTAL VARIABLES}

MOISTURE REGIME:

MESIC

NUTRIENT REGIME:

MESOTROPHIC

ELEVATION:

$606 \mathrm{M}$

SOIL DRAINAGE:

WELL

RANGELAND HEALTH RATING:

HEALTHY

FORAGE PRODUCTION (KG/HA)

$\begin{array}{ll}\text { GRASS } & 525 \\ \text { FORBS } & 100 \\ \text { SHRUBS } & 0 \\ \text { TOTAL } & 625\end{array}$

ECOLOGICALLY SUSTAINABLE STOCKING RATE $3.0 \mathrm{HA} / \mathrm{AUM}(0.15 \mathrm{AUM} / \mathrm{AC})$ 


\section{CMD7. Aw-Sw/Rose/Low forb \\ (Populus tremuloides-Picea glancal Rosa acicularis Low forb)}

$\mathbf{n = 5}$ This community type is dominated by aspen in the primary canopy and by spruce in the secondary canopy It occupies similar site conditions to the Aw Rose Low forb community type. As spruce succeeds into the canopy it reduces the amount of light reaching the forest floor reducing the growth of shrubs. fortos and grass. This community type would be rarely used by livestock and should be rated as secondary range.

\section{PLANT COMPOSITION CANOPY COVER(\%)} MEAN RANGE CONST.

\section{TREES}

WHITE SPRUCE

(Picea glauca)

TREMBLNG ASPEN

(Populus tremuioides) $\quad 33 \quad \mathbf{2 0 - 6 0} \quad 100$

BALSAM POPLAR

(Populus balsamifera) $3 \quad 6-10 \quad 40$

SHRUBS

SNOWBERRY

(Symphoricarpos occidentalis) $2 \quad 3-4 \quad 40$

PRICKI Y ROSE

$\begin{array}{llll}\text { (Rosa acicularis) } & 10 & 1-19 & 100\end{array}$

BRACTED HONEYSLCKLE

(Ioniceta imolctara) $4 \quad 4-15 \quad 40$

BUFFALOBERRY

(Shepherdia conadiensis) = $\quad 1-7 \quad 60$

FORBS

TWIFLOWER

(Linnaea borealis) $\quad 3 \quad 1-5 \quad 80$

BLNCHBERRY

(Cormus canadensis) $\quad+\quad 0-6 \quad 100$

WINTERGREEN

$\begin{array}{llll}\text { (Pyrola asarifolia) } & 1 & 0-3 & 60\end{array}$

DEWBERRY

(Rubus pubscens) = $\quad 1460$

BISHOP'S CAP

(Mitella muda)

GRASSES

HAIRY WILDRIE

(Elimus innovatus) $\quad 5 \quad 1-10 \quad 80$

MARSH REEDGRASS

(Calamagrostis canadensis) $3 \quad 1-9 \quad 60$

Mosses

Moss SPP.

\section{ENTIRONMENTAL VARLABLES}

MOISTLRE REGIM:

MESIC

NitrieNt REgIE:

MESOTROPHEC

ELEVATION:

$573(150-758) \mathrm{M}$

SOIL DRARAGE:

WELI

RANGEL AND HEALTH RATNG:

HEALTHY

FORAGE PRODUCTIOY (KG/HAF)
GR $45 S$
$5602-308$,
FORBS $194(70-418)$
SHRLBS 128(50-308)
TOTAL 408(160-1034)

\section{ECOLOGICALIY SUSTANABLE STOCNRTG RATE 4.5 HA ALN $(>0.1$ ALM AC)}




\section{CMD8. Aw-Sw/Labrador tea/Moss \\ (Populus tremuloides-Picea glauca/Ledum groenlandicum/Moss)}

$\mathbf{n}=\mathbf{1}$ This community type has relatively poor nutrient status. Labrador tea and bog cranberry are indicative of acidic soil surface soil conditions. Beckingham and Archibald (1996) described this ecosite with a jack pine and black spruce dominated overstory. The moisture and nutrient conditions of this community type are probably better than their ecosite, which allows aspen and white spruce to dominate the overstory, but the soil conditions are poorer than the Aw-Sw/Rose/Low forb community type. This community type produces little palatable forage and therefore would be classified as non-use.

\section{PLANT COMPOSITION CANOPY COVER(\%) \\ MEAN RANGE CONST.}

TREES

ASPEN

(Populus tremuloides)

WHITE SPRUCE

(Picea glauca)

SHRUBS

LABRADOR TEA

(Ledum groenlandicum.) 11

BLUEBERRY

(Vaccinium myrtilloides)

BOG CRANBERRY

(Vaccinium vitis-idaea)

FORBS

BUNCHBERRY

(Cornus canadensis)

TWINFLOWER

(Linnaea borealis)

BASTARD'S TOADFLAX

(Geocaulon lividum)

COW-WHEAT

(Melampyrum lineare)

GRASSES

HAIRY WILDRYE

(Elymus innovatus)

Mosses

Moss spp.

$\begin{array}{lll}55 & - & 100 \\ 40 & - & 100\end{array}$

100

100

100

100

100

100

100

$3 \quad-\quad 100$

$1 \quad-\quad 100$

$67 \quad-\quad 100$
ENVIRONMENTAL VARIABLES

MOISTURE REGIME:

SUBMESIC-MESIC

NUTRIENT REGIME:

SUBMESOTROPHIC-MESOTROPHIC

ELEVATION:

$333 \mathrm{M}$

SOIL DRAINAGE:

MODERATELY WELL

RANGELAND HEALTH RATING:

HEALTHY

FORAGE PRODUCTION (KG/HA)

$\begin{array}{ll}\text { GRASS } & 0 \\ \text { FORBS } & 96 \\ \text { SHRUBS } & 96 \\ \text { TOTAL } & 192\end{array}$

ECOLOGICALLY SUSTAINABLE STOCKING RATE NON-USE 


\section{CMD9. Sb/Labrador tea/Moss \\ (Picea mariana/Ledum groenlandicum/Moss)}

$\mathbf{n}=\mathbf{7} \quad$ This community type appears to be related to the bog ecosite described by Beckingham and Archibald

(1996). The bog ecosite commonly has organic soils consisting of slowly decomposing peat moss. This community type is considered non-use for livestock, due to the lack of forage and poor accessibility.

\section{PLANT COMPOSITION CANOPY COVER(\%)} MEAN RANGE CONST.

\section{TREES}

LARCH

(Larix laricina)

BLACK SPRUCE

(Picea mariana)

SHRUBS

WILLOW SPP.

(Salix spp.)

LABRADOR TEA

(Ledum groenlandicum) 29

FORBS

CLOUDBERRY

$\begin{array}{llll}\text { (Rubus chamaemorus) } & 8 & 13-35 & 38\end{array}$

HORSETAIL

(Equisetum arvense) $\quad 4 \quad 7-23 \quad 25$

DWARF SCOURING RUSH

(Equisetum scirpoides) $\quad 1 \quad 2-3 \quad 25$

GRASSES

MARSH REEDGRASS

(Calamagrostis canadensis) $3 \quad 3-10 \quad 50$

SEDGE

(Carex aurea)

WATER SEDGE

(Carex aquatilis

$\begin{array}{lll}4 & 7-14 & 38\end{array}$

$3 \quad 6-14 \quad 25$

Mosses

(Sphagnum spp)

$44 \quad 75-99 \quad 63$

\section{ENVIRONMENTAL VARIABLES}

MOISTURE REGIME:

SUBHYDRIC

NUTRIENT REGIME:

OLIGOTROPHIC

ELEVATION:

615(579-636) M

SOIL DRAINAGE:

POORLY

RANGELAND HEALTH RATING:

HEALTHY

Forage Production (KG/HA)

$\begin{array}{ll}\text { GRASS } & 52(0-192) \\ \text { FORBS } & 61(0-286) \\ \text { SHRUBS } & 91(0-200) \\ \text { TOTAL } & 228(30-678)\end{array}$

ECOLOGICALLY SUSTAINABLE STOCKING RATE

NON-USE 


\section{CMD10. Sb/Bog birch \\ (Picea mariana/Betula glandulosa)}

$\mathbf{n = 1}$ This community type is part ot the poor fen ecosite (Beckingham and Archibald 1996) because it has an intermediate nutrient regime between the bog and rich fen ecosites. Drainage on this community type is poor to very poor, but has some movement of water through the site. This community type has a well developed shrub layer and the grass layer consists mainly of marsh reedgrass and sedge species. The productivity of this type is moderate, but the high water table limits access to domestic livestock. This community would be rated as non-use.

Plant COMPOSITION CANOPY COVER(\%) MEAN RANGE CONST.

TREES

LARCH

(Larix laricina)

$10 \quad-\quad 100$

BLACK SPRUCE

(Picea mariana)

SHRUBS

$5 \quad-\quad 100$

WILLOW SPP.

(Salix spp.)

BOG BIRCH

$\begin{array}{llll}\text { (Betula glandulosa) } & 24 & - & 100\end{array}$

BLUEBERRY

(Vaccinium myrtilloides) $12 \quad$ - 100

FORBS

SMALL BOG CRANBERRY

(Oxycoccus microcarpus) $57 \quad$ - $\quad 100$

HORSETAIL

$\begin{array}{llll}\text { (Equisetum arvense) } & 2 & - & 100\end{array}$

THREE LEAVED SOLOMON'S-SEAL

(Smilicina trifolia) $\quad 5 \quad-\quad 100$

GRASSES

MARSH REEDGRASS

(Calamagrostis canadensis)6 - 100

SEDGE

(Carex aurea)

Mosses

(Sphagnum spp.)

\section{ENVIRONMENTAL VARIABLES}

MOISTURE REGIME:

SUBHYDRIC

NUTRIENT REGIME:

OLIGOTROPHIC

ELEVATION:

$576 \mathrm{M}$

SOIL DRAINAGE:

POORLY

RANGELAND HEALTH RATING:

HEALTHY

\section{Forage ProduCtION (KG/HA)}

$\begin{array}{ll}\text { GRASS } & 104 \\ \text { FORBS } & 90 \\ \text { SHRUBS } & 400 \\ \text { TOTAL } & 594\end{array}$

ECOLOGICALLY SUSTAINABLE STOCKING RATE NON-USE 


\section{CMD11. Aw-Sw/Hazelnut (Populus tremuloides-Picea glauca/Corylus cormuta)}

$\mathbf{n}=\mathbf{1}$ This is a mixedwood forest which is approaching climax. The northerly aspect of this community type has probably protected the site from past disturbance by fires and allowed the community to undergo succession. The high canopy of spruce limits the light reaching the forest floor, limiting the growth of grasses and forbs. As a result, the forage productivity of this community type is very low. This community would be considered non-use.

\section{Plant Composition Canopy Cover(\%)}

TREES

MEAN RANGE CONST.

WHITE SPRUCE

(Picea glauca)

ASPEN

(Populus tremuloides) 40

SHRUBS

HAZELNUT

(Corylus cornuta) 30

RED OSIER DOGWOOD

(Cornus stolonifera) 10

PRICKLY ROSE

(Rosa acicularis)

FORBS

WILD SARSAPARILLA

(Aralia nudicaulis)

SHOWY ASTER

(Aster conspicuus)

TWINFLOWER

(Linnaea borealis)

BUNCHBERRY

(Cornus canadensis)

Mosses

Moss SPP.
30

RANGE CONST

100

100

100

100

100

10

20

100

100

100

100

73

100

\section{ENVIRONMENTAL VARIABLES}

MOISTURE REGIME: MESIC

NUTRIENT REGIME: MESOTROPHIC

ELEVATION: $606 \mathrm{M}$

SOIL DRAINAGE: WELL

RANGELAND HEALTH RATING: HEALTHY

FORAGE PRODUCTION(KG/HA)

$\begin{array}{ll}\text { GRASS } & 0 \\ \text { FORBS } & 132 \\ \text { SHRUBS } & 74 \\ \text { TOTAL } & 206\end{array}$

ECOLOGICALLY SUSTAINABLE STOCKING RATE NON-USE 


\section{CMD12. Sw/Horsetail \\ (Picea glauca/Equisetum arvense)}

$\mathbf{n = 1}$ This community type is wet and nutrient rich. These sites are commonly found on fluvial or glaciolacustrine parent materials where flooding or seepage enhances the substrate nutrient supply. With high water tables, wet soil conditions organic matter tends to accumulate which favours the growth of horsetails. Generally horsetails are unpalatable to livestock and the wet ground conditions limit access. Consequently, this community type should be rated as non-use.

\section{PLANT COMPOSITION CANOPY COVER(\%)} MEAN RANGE CONST.

TREES

WHITE SPRUCE

(Picea glauca)

BALSAM FIR

(Abies balsamea)

SHRUBS

PRICKLY ROSE

(Rosa acicularis)

RIVER ALDER

(Alnus tenuifolia)

BRACTED HONEYSUCKLE

(Lonicera involucrata) 3

FORBS

DEWBERRY

(Rubus pubescens)

HORSETAIL

(Equisetum sylvaticum) $40 \quad$ - $\quad 100$

THREE LEAVED SOLOMONS SEAL

(Smilacina trifolia) $\quad 10 \quad-\quad 100$

BUNCHBERRY

(Cornus canadensis) $3 \quad-\quad 100$

GRASSES

MARSH REEDGRASS

(Calamagrostis canadensis) 10

100

\section{ENVIRONMENTAL VARIABLES}

MOISTURE REGIME:

HYGRIC

NUTRIENT REGIME:

PERMESOTROPHIC

ELEVATION:

$600 \mathrm{M}$

SOIL DRAINAGE: POOR TO MODERATELY WELL

RANGELAND HEALTH RATING:

HEALTHY

FORAGE PRODUCTION(KG/HA)

TOTAL 560*EsTIMATE
ECOLOGICALLY SUSTAINABLE STOCKING RATE NON-USE 


\section{Literature cited}

Adams, B. 1981. Range ecology and the impact of livestock grazing on the Peace River Slopes, Alberta. Public Lands Division, Grazing Land Management, Range Management Unit. Peace River, Alta.

Adams, B.W., G. Ehlert, C. Stone, D. Lawrence, M. Alexander, M. Willoughby, C. Hincz, D. Moisey, and A.Bogen. 2003. Rangeland Health Assessment for Grassland, Forest and Tame Pasute. Alberta Sustainable Resource Development. Public Lands Division. Edmonton. AB. Pub. No. T/044. 104pp.

Alberta Rangeland Health Task Group. 1999. Terms of Reference. Alberta Agriculture Food and Rural Development (Public Lands Division), Alberta Environment (Forest Management Division). Edmonton, AB. 49pp.

Bailey, A.W., M.G. Willoughby, R. Johansen and S. Smith. Management of Yukon Rangelands. Renewable Resources, Yukon Territorial Government, Whitehorse, Yukon. 55pp. ISBN-1-55018-138-6.

Beckingham, J. 1993. Ecosystem associations of Northern Alberta. Dept. of Environmental Protection, Alberta Forest Service, Edmonton.

Beckingham, J. and J.H. Archibald. 1996. Field guide to ecosites of Northern Alberta. Special report 5. Canadian Forest Service. Northwest Region. Edmonton, Alta.

Brierly, D., D. Downing and D.O'Leary. 1985. An integrated resource inventory of the Keg River study area. Vol. 1 and 11, Vegetation Classification, Alberta Energy and Natural Resources. Edmonton, Alta.

Corns, I.G.W. and R.M. Annas. 1986. Field guide to forest ecosystems of West-Central Alberta. Northern Forestry Center, Canadian Forestry Service, Edmonton, Alta. 251 pp.

Daubenmire, R. 1952. Forest vegetation of Northern Idaho and adjacent Washington and its bearing on concepts of vegetation classification. Ecol. Mongr. 22: 301-330.

Department of Environmental Protection. 1994. Natural Regions of Alberta. Alberta Environmental Protection. Edmonton, Alta. Pub. no.: I/531. 18pp.

Downing, D. and E. Karpuk. 1992. Aspen vegetation types of the Low Boreal Mixedwood ecoregion, East-Central Alberta. Alberta Forestry, Lands and Wildlife. Resource Information Branch. Land Information Services. Edmonton. AB. 79pp.

Downing, D. 2000. Review of Forage Data Gaps: Native range community types, Central/Dry Mixedwood Natural Subregions, Lower Foothills Subregion. Forest Range 
Assessment Project. Alberta Agriculture Food and Rural Development, Public Lands Division. St.Paul. AB. 5pp.

Gauch, H.G. 1982. Multivariate analysis in community ecology. Cambridge University Press, Cambridge, 298pp.

Hay, W.K., J.M. Veltman and R.W. Haag. 1985. An integrated resource inventory of the East Beaver Lake Assessment Area, Physical Land and Forage Classifications. Vol. 1, Alberta Energy and Natural Resources, Resource Evaluation and Planning. Edmonton, Alta.

Holechek, J.L., R.D. Pieper and C.H.Herbel. 1995. Range management principles and practices. ${ }^{\text {ed }}$. Prentice-Hall Inc. Engewood Cliff. New Jersey. Chapter 8.

Invasive plants of natural habitats in Canada. 1992. Environmental Canada, Canadian Wildlife Service. Ottawa, Canada. 111pp.

Johnson, D., L. Kershaw, A. MacKinnon and J. Pojar. 1995. Plants of the Western Boreal Forest and Aspen Parkland. Lone Pine Publishing. Edmonton. AB. 392pp.

Lane, C.T., M.G. Willoughby and M.J. Alexander. 2000. Range plant communities and carrying capacity for the Lower Foothills subregion. $3^{\text {rd }}$ approximation. Alberta Environment. Land and Forest Service. Edmonton. AB. Pub. No. T/532. 232pp.

Lodge, R.W., A. McLean and A. Johnston. 1968. Stock-poisoning plants of Western Canada. Agriculture Canada. Publication \# 1361.35pp.

Mackinnon, A. J. Pojar, and R. Coupe. 1992. Plants of Northern British Columbia. Lone Pine Publishing, Edmonton, Alta. 345pp.

Mueggler, W.F. 1988. Aspen community types of the Intermountain Region. U.S.D.A. Intermoutain Research Station. INT-250. 133pp.

Peterson, E.B. and N. M. Peterson. 1992. Ecology, management and use of aspen and balsam poplar in the prairie provinces. Northern Forestry Center, Canadian Forest Service. Edmonton, AB. Special report 1.252pp.

Range Survey Manual. 1992. Range Management Section, Alberta Forest Service. Edmonton, Alta. 39pp.

Strong, W.L. and J.M. Thompson. 1995. Ecodistricts of Alberta: Summary of Biophysical Attributes. Alberta Environmental Protection, Resource Data Division. Edmonton, Alta. Pub. no. T/319. 91pp.

Strong, W.L. and K.R. Leggat. 1992. Ecoregions of Alberta. Alberta Forestry, Lands and 
Wildlife, Resource Information Branch, Edmonton, Alta. T/245. 77pp.

Strong, W.L.1992. Ecoregions and Ecodistricts of Alberta. Alberta, Forestry Lands and Wildlife. Land Information Services Division. Resource Information Branch. Edmonton, Alta. Pub. no. T/244, 77pp.

Task Group on Unity and Concept. 1995. New concepts for assessment of rangeland condition. J. Range Manage. 38:220-225.

Thompson, W.H. and P.L. Hansen. 2002. Classification and management of riparian and wetland sites of the Alberta Grassland Natural Region and adjacent subregions. Bitterroot Restoration Inc. Prepared for the Alberta Riparian Habitat Management Program-Cows and Fish, Lethbridge, Alberta. 416pp.

Wilkinson, K. 1990. Trees and shrubs of Alberta. Lone Pine Publishing. Edmonton, Alta. $191 \mathrm{pp}$.

Wilkinson, K. and E.A. Johnson. 1982. Distribution of prairies and solonetzic soils in the Peace River district, Alberta. Can. J. Bot. 61: 1851-1860.

Willoughby, M.G. and D. Downing. 1995. Deciduous plant communities and carrying capacity of the Boreal Ecoprovince of Alberta. Alberta Environmental Protection. Edmonton, Alta. Pub. no. T/312. 329pp.

Willoughby, M.G. 1996. The effects of grazing on deciduous plant communities in the Boreal Ecoprovince of Alberta. Proceedings of the Fifth Int'l Rangeland Congress, Salt Lake City, Utah. Vol. 1. 610-611. 


